\title{
Lie algebras of linear operators on locally convex spaces
}

\author{
Rodrigo Augusto Higo Mafra Cabral
}

Tese de Doutorado

Orientador: Frank Michael Forger

Programa de Pós-Graduação em Matemática Aplicada

Instituto de Matemática e Estatística da

Universidade de São Paulo (IME - USP)

Trabalho produzido com apoio financeiro da agência $\mathrm{CNPq}$

Outubro de 2019 



\section{Lie algebras of linear operators on locally convex spaces}

Esta versão da tese contém as correções e alterações sugeridas pela Comissão Julgadora no dia da defesa da versão original do trabalho, realizada em 15/03/2019. Uma cópia da versão original está disponível no Instituto de Matemática e Estatística da Universidade de São Paulo.

Comissão Julgadora:

- Prof. Dr. Frank Michael Forger (orientador) - IME - USP

- Prof. Dr. Christian Dieter Jäkel - IME - USP

- Prof. Dr. Severino Toscano do Rêgo Melo - IME - USP

- Prof. Dr. Pedro Lauridsen Ribeiro - CMCC - UFABC

- Prof. Dr. Luiz Roberto Hartmann Junior - DM - UFSCar 

Dedicated to my parents,
Ione and Juarez 



\section{Agradecimentos}

Primeiramente, gostaria de agradecer aos meus pais, Ione e Juarez, pelo apoio constante e incondicional, e sem os quais este trabalho não seria possível; ao meu irmão, Gabriel, por ser um grande amigo com quem sempre posso contar; e à Gabs, por ter sido uma grande companhia nas (muitas) madrugadas em que esta tese foi escrita.

Ao meu orientador, Frank Michael Forger, por sempre compartilhar seus conhecimentos científicos de maneira tão generosa, e por expor sua visão sobre a Ciência sempre de maneira franca, entusiasmada e, certamente, inspiradora. Agradeço imensamente por ter me apresentado a uma área tão rica e fascinante dentro da Análise Funcional, e por ter tido a liberdade de estudar quaisquer tópicos que me interessassem para, somente então, decidir o tema da tese. Esta abordagem heterodoxa constituiu-se numa experiência muito enriquecedora, para mim.

Ao Severino Toscano do R. Melo, por seu grande altruísmo, pelas inúmeras conversas, sobre Matemática ou não, e pelos incontáveis ensinamentos que tanto contribuíram para a minha

formação de Matemático, como aluno, orientando e, agora, colaborador. É realmente impossível quantificar o aprendizado que obtive durante todos esses anos.

Ao professor Paulo Domingos Cordaro, por poder ter assistido como ouvinte o excelente curso "Espaços Localmente Convexos e Aplicações", ministrado em 2017, e pelos esclarecimentos acerca de operadores fortemente elípticos.

Ao Eric Ossami Endo, por sempre me incentivar a expor o meu trabalho, pelos convites a tantos seminários e eventos e pelas várias aulas de Mecânica Estatística Clássica.

Ao Lucas Affonso, por sempre me mostrar alguma aplicação (ou possível aplicação!) interessante de Análise Funcional à Mecânica Estatística Quântica. 



\section{Resumo}

Palavras-chave: álgebras de Lie, grupos de Lie, representações fortemente contínuas, exponenciação, espaços localmente convexos, limites projetivos, limites inversos, vetores analíticos projetivos, álgebras localmente convexas, *-álgebras localmente convexas, álgebras localmente $\mathrm{C}^{*}$, álgebras de Arens-Michael, *-álgebras de Arens-Michael, álgebras de von Neumann, álgebras GB*, automorfismos, *-automorfismos, derivações, *-derivações, operadores pseudo-diferenciais.

Condições necessárias e suficientes para a exponenciação de álgebras de Lie reais de dimensão finita de operadores lineares sobre espaços localmente convexos completos Hausdorff são obtidas, com foco no caso equicontínuo - em particular, condições necessárias para a exponenciação com respeito a grupos de Lie compactos são estabelecidas. Aplicações para álgebras localmente convexas completas são dadas, com uma atenção especial para álgebras localmente $\mathrm{C}^{*}$. A definição de vetor analítico projetivo é introduzida, possuindo um papel importante em alguns dos teoremas de exponenciação e na caracterização dos geradores de uma certa classe de grupos a um parâmetro fortemente contínuos. 



\section{Abstract}

Keywords: Lie algebras, Lie groups, strongly continuous representations, exponentiation, locally convex spaces, projective limits, inverse limits, projective analytic vectors, locally convex algebras, locally convex *-algebras, locally $\mathrm{C}^{*}$-algebras, pro-C ${ }^{*}$-algebras, LMC*-algebras, Arens-Michael algebras, Arens-Michael *-algebras, von Neumann algebras, GB*-algebras, automorphisms, *-automorphisms, derivations, *-derivations, pseudodifferential operators.

Necessary and sufficient conditions for the exponentiation of finite-dimensional real Lie algebras of linear operators on complete Hausdorff locally convex spaces are obtained, focused on the equicontinuous case - in particular, necessary conditions for exponentiation to compact Lie groups are established. Applications to complete locally convex algebras, with special attention to locally $\mathrm{C}^{*}$-algebras, are given. The definition of a projective analytic vector is given, playing an important role in some of the exponentiation theorems and in the characterization of the generators of a certain class of strongly continuous oneparameter groups. 



\section{Contents}

Introduction $\quad$ vii

1 Preliminaries 1

1.1 One-Parameter Semigroups and Groups . . . . . . . . . . . . . . . . . 3

1.2 Lie Group Representations and Infinitesimal Generators . . . . . . . . . . 8

1.2.1 The Gårding Subspace . . . . . . . . . . . . . . . . . . . . . . . . . . 12

1.2.2 The Space of Smooth Vectors is Left Invariant by the Generators . . 16

1.2.3 Lie Algebra Representations Induced by Group Representations . . . 18

1.2.4 Group Invariance and Cores . . . . . . . . . . . . . . . . . . . 19

1.3 Dissipative and Conservative Operators . . . . . . . . . . . . . . . 27

1.4 The Kernel Invariance Property (KIP),

Projective Analytic Vectors . . . . . . . . . . . . . . . . . . 30

1.5 Some Estimates Involving Lie Algebras . . . . . . . . . . . . . . . . 45

1.6 Extending Continuous Linear Maps . . . . . . . . . . . . . . . . . . . . 49

1.7 Projective Limits . . . . . . . . . . . . . . . . . . 50

2 Group Invariance and Exponentiation $\quad \mathbf{5 7}$

Constructing a Group Invariant Domain . . . . . . . . . . . . . . . . . 57

Exponentiation Theorems . . . . . . . . . . . . . 86

The First Exponentiation Theorems . . . . . . . . . . . . . . . 86

Strongly Elliptic Operators - Sufficient Conditions for Exponentiation . . . 97

Strongly Elliptic Operators - Necessary Conditions for Exponentiation . . . 116

Exponentiation in Locally Convex Spaces - Characterization . . . . . . . . . 120

3 Some Applications to Locally Convex Algebras 123

Definitions, Examples and a Few Structure Theorems . . . . . . . . . . . . . . . 123

Exponentiation of Complete Locally Convex Algebras . . . . . . . . . . . . . . 133 
Exponentiation of Locally $\mathrm{C}^{*}$-Algebras . . . . . . . . . . . . . . 136

$\begin{array}{ll}\text { Bibliography } & 139\end{array}$ 


\section{Introduction}

\section{A Physics Point of View: Some Motivations ${ }^{1}$}

With the advent of Quantum Theory, the theory of Lie groups and their representations has assumed an important role in Physics, mainly to mathematically incorporate the notion of symmetry. Some highlights that could be mentioned are, already in the 1930's, the development of the theory of compact Lie groups and their representations, by Hermann Weyl, a typical application being the consequences of rotational symmetry in atomic spectroscopy, and the classification of relativistic elementary particles in terms of irreducible unitary representations of the Poincaré group, by Eugene Wigner. Another historical landmark was the "eightfold way", by Gell'Mann and Ne'eman, in the 1960's, to classify hadrons (strongly interacting particles) in terms of weight diagrams of the group $S U(3)$.

In the early stages of Lie group representation theory, studies dealt almost exclusively with unitary representations on Hilbert spaces as the state spaces of quantum systems. However, Quantum Mechanics had already exposed with clarity a phenomenon which is present even in Classical Mechanics, but had rarely been treated there in an explicit manner: the duality between the state space and the algebra of observables of a system, which leads, in the treatment of temporal evolution, to the distinction between the "Schrödinger picture" (with time-dependent states and static observables) and the "Heisenberg picture" (with static states and time-dependent observables). The transition from Quantum Mechanics to Quantum Field Theory strongly suggests that it is, at the very least, more convenient to perform this temporal evolution - or, more generally, the dynamics - on the observables, and not on the states, since the observables, and not the states, are the ones that allow localization in regions of space-time. Hence, besides unitary representations on

\footnotetext{
${ }^{1}$ The author would like to thank his advisor, Frank Michael Forger, for the big help in the writing process of this section of the Introduction. His great knowledge of Physics contributed in an essential way to the final form of the text.
} 
Hilbert spaces one should also investigate representations by automorphisms on algebras of observables. The question that naturally arises is then: which kind of algebras must one use?

A first answer to this question may be found in Algebraic Quantum Field Theory, initiated in 1964 by Haag and Kastler, which revolves around a central concept that is absent in Quantum Mechanics: locality. This is incorporated by demanding the existence of a net of local $\mathrm{C}^{*}$-algebras $\mathcal{A}(U)$ associated to an adequate (suficiently large) family of bounded open sets $U$ in space-time satisfying the property that, if $U \subset V$, then $\mathcal{A}(U) \subset$ $\mathcal{A}(V)$. The total $\mathrm{C}^{*}$-algebra of this net is defined by

$$
\mathcal{A}:=\overline{\bigcup_{U} \mathcal{A}(U)}
$$

where the closure indicates the $\mathrm{C}^{*}$-completion of the algebra. Relativistic invariance of the theory is implemented by a representation $\alpha: \mathcal{P} \longrightarrow \operatorname{Aut}(\mathcal{A})$ of the Poincaré group $\mathcal{P}$ by *-automorphisms of $\mathcal{A}$ which is compatible with this net:

$$
\alpha(a, \Lambda)[\mathcal{A}(U)]=\mathcal{A}(\Lambda U+a) .
$$

Locality means that $\mathcal{A}(U)$ and $\mathcal{A}(V)$ commute when the two regions $U$ and $V$ are spatially separated [62].

However, since the beginning of this theory proposed by Haag and Kastler, there exists a discussion on the question of what would be the exact nature of the algebras $\mathcal{A}(U)$ involved: $\mathrm{C}^{*}$-algebras, von Neumann algebras or some other type of locally convex algebras? ${ }^{2}$ And which would be the dense $*$-subalgebras serving as natural domains for the generators of the one-parameter groups referring to the representation $\alpha$ (or even to representations of another Lie group that perhaps arises in the theory)?

\section{A Mathematical Point of View}

Infinitesimal generators are mathematical objects which appear within the context of locally convex spaces as particular kinds of linear operators, defined from an action of the additive semigroup $[0,+\infty)$ (in the case of semigroups) or the additive group of real numbers (in the case of one-parameter groups) on the subjacent space - see Section 1.2. This action is usually implemented by continuous linear operators and subject to a condition of continuity: it must be continuous with respect to the usual topology of $[0,+\infty)$ (or $\mathbb{R}$,

\footnotetext{
${ }^{2}$ Nowadays, there seems to be a consensus about this, a fact which the author learned from professor Christian D. Jäkel: such local algebras must be type $\mathrm{III}_{1}$ approximately finite-dimensional factors with separable predual [125, page 137]; by a result of Haagerup [63] there exists, up to equivalence, only one such algebra - see, also, [38, page 74$]$.
} 
in the group case) and a fixed locally convex topology on the space of continuous linear operators (usually, at least in the infinite-dimensional framework, there are several options for this choice). One of the possible choices gives rise to strongly continuous semigroups and strongly continuous groups, and will be the topology of choice for the investigations in this work.

Within the normed context, there exist two very important classes of such strongly continuous actions: contraction semigroups and groups of isometries. In Hilbert space theory, for example, strongly continuous one-parameter groups of isometries are implemented by unitary operators and, according to the Spectral Theorem for self-adjoint operators and Stone's theorem (see Theorems VIII.7 and VIII.8, of [104]), their generators are precisely the anti-adjoint operators (in other words, operators such that $A^{*}=-A$ ). The Feller-Miyadera-Phillips Theorem (see Section 1.2) characterizes the generators of strongly continuous one-parameter (semi)groups on Banach spaces. When specialized to the contractive and to the isometric cases, it yields the famous Hille-Yosida Theorem. Also in this context there is the Lumer-Phillips Theorem which, for groups of isometries, states that the infinitesimal generators must be conservative linear operators whose perturbations by non-zero multiples of the identity are surjective (these two results are also explicitly stated in Section 1.2). This is a direct generalization of Stone's Theorem to Banach spaces, since anti-symmetric (or anti-hermitian, if one prefers) operators are conservative and the selfadjointness property for such operators is given by an analogous surjectivity condition (see [104, Theorem VIII.3], or [32, Theorem II, page 24]).

For more general complete locally convex spaces, there is a theorem which characterizes the generators of equicontinuous semigroups in an analogous way that the Lumer-Phillips Theorem does [3, Theorem 3.14]. Also in this more general setting, references [8, Theorem 4.2] and [8, Corollary 4.5] give characterizations in the same spirit that the Feller-MiyaderaPhillips and Hille-Yosida Theorems do, respectively. These three theorems were of great importance for the development of this work, and their detailed statements may be found in Section 1.4.

When the locally convex space under consideration has some additional algebraic structure, turning it into an algebra (respectively, a *-algebra), for example, the actions of interest are by strongly continuous one-parameter groups of automorphisms (respectively, *automorphisms) and their generators then become derivations (respectively, *-derivations), since they satisfy the Leibniz product rule. Algebras will appear only in Chapter 3.

There is another very important direction of generalization, which consists in passing from one-parameter groups to more general groups. One framework that comes to mind here would be to consider abstract topological groups and their actions on locally convex spaces. But often, the study of smooth and analytic elements (see Section 1.2) of a given action is very important and useful, so a natural requirement on the group is that it should be a Lie group, becoming, in this way, a smooth manifold - in reality, it can then be 
proved that it actually possesses the structure of an analytic manifold. One motivation for this interest, for example, may be found in the content exposed at the beginning of the first section of this Introduction. Also, it is very important that the group in question is not restricted to be commutative. Classical results in this direction, in the Hilbert and Banach space context, may be found in the influential paper [94] of Edward Nelson. Two of its theorems state that every strongly continuous Lie group representation has a dense subspace of analytic vectors when the representation is by unitary operators on a Hilbert space [94, Theorem 3] and, more generally, when the representation is by bounded linear operators on a Banach space [94, Theorem 4]. This paper, whose study was suggested by the author's advisor, was the starting point for this research and, in one of the discussions, it was suggested that the author investigate what was the actual role that the generalized Laplacian defined in [94] played in some of its results. ${ }^{3}$

Conversely, one may investigate the exponentiability of a (real finite-dimensional) Lie algebra $\mathcal{L}$ of linear operators: when can its elements be obtained (as generators of oneparameter groups) from a strongly continuous representation $V$ of a (connected) Lie group $G$, having a Lie algebra $\mathfrak{g}$ isomorphic to $\mathcal{L}$ via $\eta: \mathfrak{g} \longrightarrow \mathcal{L}$, according to the formula

$$
\left.\frac{d}{d t} V(\exp t X)(x)\right|_{t=0}=\eta(X)(x), \quad X \in \mathfrak{g}, x \in \mathcal{D},
$$

where $\exp : \mathfrak{g} \longrightarrow G$ is the corresponding exponential map?

For representations on finite-dimensional vector spaces, a classical theorem states that every Lie algebra of linear operators is exponentiable, provided only that one chooses $G$ to be simply connected ${ }^{4}$ (see the brief discussion after Definition 2.6).

In the infinite-dimensional case, things are much more complicated, and when formulating the question some reasonable a priori requirements are usually assumed: the elements of $\mathcal{L}$ are linear operators defined on a common, dense domain $\mathcal{D}$ which they all map into itself. But there are counterexamples showing that these are not sufficient: one can find linear operators satisfying all these conditions whose closures generate strongly continuous one-parameter groups, but the closures of certain of their real linear combinations (including perturbations of one by the other), or of their commutator, do not - see the result stated at the end of Section 10 of [94]; see also [25, Example 4.1]. Finding sufficient criteria for exponentiability is thus an intricate problem because these must exclude such unpleasant situations. One of the first important results in this direction, for Lie algebras of anti-symmetric (anti-hermitian) operators on Hilbert spaces, can be found in [94, Theorem $5]$.

\footnotetext{
${ }^{3}$ The author would like to thank his advisor, Frank Michael Forger, for suggesting the study of Lie group representations in the context of locally convex *-algebras. The author would also like to thank Professor Severino Toscano do R. Melo for the many discussions on the papers [94] and [65] in the early stages of this work, and also for his teachings on the theory of pseudodifferential operators.

${ }^{4} \mathrm{~A}$ topological space $\mathcal{X}$ is said to be simply connected if it is path-connected - so, in particular, it is connected - and if every continuous curve $\gamma:[0,1] \longrightarrow \mathcal{X}$ satisfying $\gamma(0)=\gamma(1)=: x_{0}$ (this is called a loop based at $\left.x_{0}\right)$ is path homotopic to the constant curve $\gamma_{0}:[0,1] \ni t \longmapsto x_{0}$ (see [92, page 333]).
} 
As for representations by $*$-automorphisms of $\mathrm{C}^{*}$-algebras, there exist several studies in the literature. For example, in [27] and [28], generation theorems - in other words, theorems which give necessary and (or) sufficient conditions on a linear operator in order for it to be the generator of some one-parameter semigroup or group - for $*$-derivations, which stem from different kinds of hypotheses, are investigated. In the more general subject of Lie groups, the exponentiation theorem [25, Theorem 3.9] is redirected to the more specific context of representations on $\mathrm{C}^{*}$-algebras, in [26]. It should be mentioned that reference [25], along with [74] and [3], have been three of the most inspiring works for this thesis. Other references regarding Lie group representations by $*$-automorphisms on $\mathrm{C}^{*}$-algebras are [24], [30], [99], [114] and [116].

An aspect which helps when comparing the question of exponentiability in different contexts is that there is a close relation between Lie group representations by unitary operators on Hilbert spaces and by $*$-automorphisms on $\mathrm{C}^{*}$-algebras.

For example, if $\mathcal{H}$ is a Hilbert space and $U: G \longrightarrow \mathcal{B}(\mathcal{H})$ is a strongly continuous representation of a Lie group $G$ by unitary operators, then the "adjoint of $U$ ", $\operatorname{Ad} U: G \longrightarrow$ $\operatorname{Aut}(\mathcal{B}(\mathcal{H}))$, defined by

$$
(\operatorname{Ad} U)(g)(a):=U(g) a U(g)^{-1}, \quad g \in G, a \in \mathcal{A},
$$

is a representation of $G$ by $*$-automorphisms of the $\mathrm{C}^{*}$-algebra $\mathcal{B}(\mathcal{H})$. However, in general, this one is strongly continuous only on an $\mathrm{Ad} U$-invariant $\mathrm{C}^{*}$-subalgebra of $\mathcal{B}(\mathcal{H})$, denominated the $C^{*}$-subalgebra of continuous elements of the representation $\mathrm{Ad} U$. For example, for the Canonical Commutation Relations in the Weyl form, as a unitary representation $U$ of the Heisenberg group, the continuous elements of the representation Ad $U$ form a proper $\mathrm{C}^{*}$-subalgebra of $\mathcal{B}\left(L^{2}\left(\mathbb{R}^{n}\right)\right)$, as noted at the bottom of page 248 of [42].

Conversely, let $\alpha: G \longrightarrow \operatorname{Aut}(\mathcal{A})$ be a strongly continuous representation of $G$ by *automorphisms on a $\mathrm{C}^{*}$-algebra $\mathcal{A}$, and let $w$ be a state on $\mathcal{A}$ which is $G$-invariant, that is, a state satisfying

$$
w \circ \alpha_{g}=w, \quad g \in G .
$$

This implies that the kernel of $w$,

$$
\text { Ker } w:=\left\{a \in \mathcal{A}: w\left(a^{*} a\right)=0\right\}
$$

is also $G$-invariant:

$$
\alpha_{g}[\operatorname{Ker} w] \subseteq \operatorname{Ker} w, \quad g \in G .
$$

Then, one may define a strongly continuous representation of $G$ by unitary operators on the GNS representation Hilbert space $\mathcal{H}_{w}$ by

$$
U_{\alpha}: G \longrightarrow \mathcal{B}\left(\mathcal{H}_{w}\right), \quad U_{\alpha}(g)([a]):=\left[\alpha_{g}(a)\right], \quad g \in G, a \in \mathcal{A},
$$

where $[a] \in \mathcal{H}_{w}$ denotes the equivalence class of $a \in \mathcal{A}$ (these equivalence classes form a dense subspace of $\mathcal{H}_{w}$ ). 
The constructions mentioned in the last two paragraphs, connecting the theory of representations on Hilbert spaces and on $\mathrm{C}^{*}$-algebras, are just another instance of how these two theories are interconnected, a phenomenon which is also apparent in other contexts: the Spectral Theorem for normal operators ([32, page 116]), the Gelfand-Naimark Theorem ([93, page 94]), the theory of Hilbert $\mathrm{C}^{*}$-modules $([79])$, and others. No wonder that it is a recurring habit to elaborate parallels between these two theories to motivate results of one of them through the other.

\section{Main Objectives}

The main objective of this thesis is to obtain some new results regarding the exponentiation of (in general, noncommutative) finite-dimensional real Lie algebras of linear operators acting on complete Hausdorff locally convex spaces, focusing on the equicontinuous case, and to search for applications within the realm of locally convex algebras. To the knowledge of the author, there are almost no theorems in the literature dealing with the exponentiability of Lie algebras of dimension $d>1$ of linear operators on locally convex spaces beyond the context of Banach spaces ([74, Theorem 9.1, page 196] would be such an example). There exist results for $d=1$ (see [3], [8], [78] and [97], for example), but the technical obstructions to achieve exponentiability in this context for $d>1$ are considerably more severe. In order to accomplish this, it must first be shown how to construct a group invariant dense $\mathrm{C}^{\infty}$ domain from a mere dense $\mathrm{C}^{\infty}$ domain. To this end, techniques developed in Chapters 5, 6 and 7 of [74] in the Banach space context must be suitably adapted. Then, "locally convex equicontinuous versions" of three exponentiation theorems found in the literature ([74, Theorem 9.2], [59, Theorem 3.1] and [25, Theorem 3.9]) are proved. Considerable upgrades on this last reference are made: besides the extension to the vastly more general framework of complete Hausdorff locally convex spaces, deeper studies on the role of the generalized Laplacian employed in [25] are performed, in the course of which it is substituted by an arbitrary strongly elliptic operator in the (complexification of the) universal enveloping algebra of the operator Lie algebra under consideration. In Theorem 2.14, a characterization of exponentiability in complete Hausdorff locally convex spaces in the same spirit as in [25, Theorem 3.9] is given, with the exception that, in the present work, arbitrary strongly elliptic operators are considered. Since the subjacent Lie group representations are locally equicontinuous, this theorem gives, in particular, necessary conditions for exponentiation with respect to compact Lie groups. In Chapter 3, some applications to complete locally convex algebras are given (Theorem 3.7), with special attention to locally $\mathbf{C}^{*}$-algebras (Theorems 3.8 and 3.9). Some simple theorems on structural aspects of certain types of locally convex *-algebras are also obtained (Corollary 3.4 is a good example of this). Moreover, in Chapter 1 (more precisely, in Theorem 1.4.8), a characterization of the generators of a certain class of strongly continuous one-parameter groups is established, in which the existence of a dense set of projective analytic vectors plays a central role - see Definition 1.4.2. In Example 1.4.10, 
this theorem is invoked in the context of strongly continuous Lie group representations on Banach spaces, and applied to concrete algebras of functions and of pseudodifferential operators. 


\section{Structure of the Thesis}

Chapter One: this chapter is intended, for the most part, to introduce the basic concepts and objects that appear in this work.

The chapter begins by listing a few structural facts about locally convex spaces and defining some important kinds of strongly continuous semigroups and groups. Section 1.2, which composes substantial part of the Preliminaries, contains several useful results on infinitesimal generators, beginning with the important definitions of smooth and analytic vectors, already in the context of locally convex spaces. Subsection 1.2.1 is entirely devoted to the precise definition of a very important subspace of smooth vectors known as the Gårding Domain, or Gårding subspace, since a rigorous treatment of the basic technical issue here does not seem to be readily available in the literature - the elements of this subspace are defined by integrals over a Hausdorff locally compact group assuming values in a locally convex space, so some technical concepts, like wafer-completeness and dual topologies, have to be carefully manipulated. Also, it is shown that this subspace is group invariant and is dense, thus proving the useful corollary that the space of smooth vectors is also dense in the representation space under consideration. Subsection 1.2.2 gives a simpler characterization of the subspace of smooth vectors for wafer-complete Hausdorff locally convex spaces, based on an argument that can be found in [58], and the corollary that this subspace is left invariant by the generators associated to a strongly continuous Lie group representation is obtained. Subsection 1.2.3 shows how a Lie algebra representation arises from a strongly continuous Lie group representation, and Subsection 1.2.4 introduces the important notions of a core for a closed operator and of group invariant domains. In particular, the very useful corollary that the space of smooth vectors is a group invariant core domain for the generators associated to a strongly continuous locally equicontinuous representation is obtained - see Theorem 1.2.4.1 and Corollary 1.2.4.2. A statement which is similar to that of Theorem 1.2.4.1 is claimed in [74, Theorem B.5, page 446], but not proved. Moreover, it is claimed there under the hypothesis of sequential completeness, but the author of this thesis found it necessary to add the hypothesis that it is also wafer complete - due to a pragmatical reason, the hypothesis of completeness, instead of wafer completeness + sequential completeness, was assumed by the author. Lemma 1.2.4.3 is also an adaptation to locally convex spaces of a known Banach space theorem [30, Corollary 3.1.7, page 167].

The next task is to introduce the delicate notions of dissipative and conservative operators: this is done in Section 1.3. The delicacy here is due to the fact that these properties depend on the particular choice of the fundamental system of seminorms for the space see [3, Remark 3.10]. The Kernel Invariance Property for linear operators, abbreviated as (KIP), is then defined in Section 1.4. It was not known to the author, at the time when this concept was defined, that this notion had already appeared in [8], where the term "compartmentalized operators" was used. This notion is fundamental for the entire thesis, 
permeating all of Chapter 2, and also Chapter 3: a recurring strategy used throughout the thesis consists in implementing the (KIP) to reduce a locally convex problem to an infinite number of Banach space problems, solve these and then try to "glue" this infinite number of solutions together to solve the original locally convex problem. A very symbolic example illustrating this process is the proof of Theorem 2.3 where, after the (KIP) is explored, Gelfand's spectral radius formula is repeatedly used on Banach algebras of operators. Still in Section 1.4, three generation theorems for complete Hausdorff locally convex spaces, which are proved in [3] and [8], are stated. Also, the definition of projective analytic vectors (Definition 1.4.2), which is going to be very useful in the proofs of the Exponentiation Theorems of Chapter 2, is given by the author. First, two lemmas (1.4.1 and 1.4.3) are proved, ${ }^{5}$ and Observation 1.4.1.2 motivates the author to define the notion of a Hausdorff locally convex space with complete quotients. Then, generation theorems for $\Gamma$-groups (defined in Section 1.1) are given, in which the existence of a dense set of projective analytic vectors plays a central role. Ultimately, they lead to a characterization of generators of $\Gamma$-groups, in Theorem 1.4.8, and a special application to equicontinuous groups, in Theorem 1.4.9. An application to strongly continuous Lie group representations on Banach spaces is also given in Example 1.4.10, with a focused analysis on concrete algebras of functions and of pseudodifferential operators. ${ }^{6}$

Section 1.5 proves some simple, yet very important estimates, involving differential seminorms defined from a fixed basis of a finite-dimensional real Lie algebra of linear operators. Then, Lemma 1.5.1 uses them to prove a key formula for one of the main exponentiation theorems of the thesis. This formula appears in the proof of [25, Lemma 2.3] (under the name of "Duhamel formula") and in [109, page 80], but in both cases, without proof. This absence of a proof, combined with the lack of familiarity of the author with such an identity, motivated the existence of Lemma 1.5.1. In Section 1.6, a lemma which is well-known in the realm of normed spaces ${ }^{7}$ but not so popular in that of locally convex spaces, is proved in this more general setting, for the sake of completeness - it is strongly used in the proof of Theorem 2.2, for example. Finally, in Section 1.7, the projective limit of Hausdorff locally convex spaces is defined, examples are given and some results are proved: among them is the well-known theorem which states that a complete Hausdorff locally convex space is always isomorphic to a projective limit of Banach spaces (Lemma 1.7.1).

Chapter Two: this chapter is divided into two big sections. The first one deals with

\footnotetext{
${ }^{5}$ It should be mentioned that these two lemmas are key ingredients for the proofs contained in Chapter 2, and that Lemma 1.4.3 is a locally convex version of a very well-known generation theorem for Banach spaces, [111, Theorem 3].

${ }^{6}$ The proofs from Theorem 1.4.4 to Example 1.4.10 use a lot of the content exposed in Section 1.7, so the reader may want to take a look at that section before proceeding. It should also be emphasized that these theorems are not required to prove the exponentiation theorems of Sections 2 and 3.

${ }^{7}$ See the BLT Theorem in [104, Theorem I.7, page 9].
} 
the problem of constructing a larger, group invariant dense $\mathrm{C}^{\infty}$ domain from a given dense $\mathrm{C}^{\infty}$ domain associated to a finite-dimensional real Lie algebra of linear operators. After giving a more explicit characterization of the maximal $\mathrm{C}^{\infty}$ domain for a finite set of closed linear operators and introducing some technical and very important definitions - like the augmented spectrum and the diminished resolvent of a linear operator and the $\mathbf{C}^{\mathbf{1}}$-closure $\mathcal{D}_{1}$ of a dense $\mathbf{C}^{\infty}$ domain $\mathcal{D}$ - the construction of the desired group invariant domain begins as an escalade which is divided into five parts. Theorem 2.1 is about obtaining commutation identities involving generators and their resolvent operators and, to this end, inductive proofs using finite-dimensional linear algebra take care of the problem. These identities will be the basis for the next four theorems. Theorem 2.2 proves that the resolvents of the generators in question restrict to continuous (with respect to the finer $\mathrm{C}^{1}$-topology) linear operators acting on $\mathcal{D}_{1}$, while Theorem 2.3 obtains a commutation relation via a series expansion. Theorem 2.4 then uses this identity to prove that the one-parameter groups in question, just like the resolvent operators, restrict to strongly continuous representations by continuous (also with respect to the $\mathrm{C}^{1}$-topology) linear operators on $\mathcal{D}_{1}$. Also, a commutation-type identity via a series expansion, involving the restricted one-parameter groups and a particular extension of the pregenerators, is proved. This identity is very important to make the proof of Theorem 2.5 work, which concludes the construction of the group invariant domain. A few other interesting results are obtained in Theorem 2.5, such as the fact that the one-parameter groups in question respect the differential structure of all orders; in other words, the one-parameter groups, when restricted to the $\mathrm{C}^{n}$-closures of $\mathcal{D}, n \geq 1$, and to the smooth closure of $\mathcal{D}$, leave these subspaces invariant, and act there as one-parameter groups of the kinds defined in [8] (they are also properly defined in Section 1.1 of this thesis). Another aspect which is noteworthy is that a necessary condition for the construction to succeed is that the basis elements must satisfy a kind of "joint (KIP)" condition with respect to a certain fundamental system of seminorms associated to a generating set (in the sense of Lie algebras) of the operator Lie algebra, which is composed of pregenerators of equicontinuous groups. This is an aesthetically ugly hypothesis, but it will naturally disappear in the statements of the main exponentiation theorems of the thesis.

Finally, Section 2.2 contains the core results of this work: the exponentiability theorems for finite-dimensional real Lie algebras of linear operators on complete Hausdorff locally convex spaces, in which equicontinuity plays a central role. Many of those theorems are generalizations of results which can be found in the literature: for example, "locally convex equicontinuous versions" of Ref. [74, Theorem 9.2] (in Theorem 2.7 and Corollary 2.8), Ref. [59, Theorem 3.1] (in Theorem 2.9 and Corollary 2.10) and Ref. [25, Theorem 3.9] (in Theorem 2.12), are proved. Actually, Theorem 2.12 is one of the most important results in this thesis. Apart from the extension to the locally convex setting, it contains other improvements as compared to [25, Theorem 3.9]. For example, the implication $(\Rightarrow)$ there is reobtained for an arbitrary dense core domain, and not only for the maximal $\mathrm{C}^{\infty}$-domain. 
Also, as already mentioned before, it is established for a general strongly elliptic operator in the (complexification of the) universal enveloping algebra of the operator Lie algebra under consideration (the concept of a strongly elliptic operator is defined right after Corollary 2.10, and a motivation from PDE theory is also included).

For didactic reasons, the characterization of exponentiable real finite-dimensional Lie algebras of linear operators is broken down into three parts: Theorem 2.11 establishes sufficient conditions for exponentiation in Banach spaces, with an arbitrary dense core domain and a general strongly elliptic operator. Then, Theorem 2.12 reobtains this same result for complete Hausdorff locally convex spaces, and Theorem 2.13 gives necessary conditions for exponentiability in the same context - Observation 2.13.1 slightly strengthens it for compact Lie groups -, but restricted to the case where the dense core domain is the maximal $\mathrm{C}^{\infty}$-domain. Finally, in Theorem 2.14, a characterization of exponentiability in complete Hausdorff locally convex spaces in the same spirit as in $[25$, Theorem 3.9] is given, but with general strongly elliptic operators being considered.

Chapter Three: at the beginning of this final chapter, various definitions revolving around basic concepts from the theory of locally convex algebras and locally convex *algebras are assembled. Then, some examples of locally convex *-algebras are given: ArensMichael *-algebras, locally $\mathrm{C}^{*}$-algebras, $\mathrm{C}^{*}$-like locally convex *-algebras, $\mathrm{GB}^{*}$-algebras and von Neumann algebras. Theorem 3.2 proves that locally convex $*$-algebras satisfying certain properties will have complete quotients, a concept introduced in Section 1.4 in the context of locally convex spaces. Two nice corollaries arise: Corollary 3.3 shows that, given a locally convex $*$-algebra, the apparently weaker hypothesis that the kernels of the seminorms in a fundamental system should be ideals is actually equivalent to the fact that the algebra is a locally convex $\mathrm{m}^{*}$-algebra; on the other hand, Corollary 3.4 gives the interesting conclusion that the only complete $\mathrm{GB}^{*}$-algebras which are $\mathrm{m}^{*}$-convex algebras are the locally $\mathrm{C}^{*}$-algebras. ${ }^{8}$

Returning to the exponentiation issue, Lemma 3.5 proves that, once a finite-dimensional real Lie algebra of derivations (respectively, *-derivations) on a locally convex algebra (respectively, locally convex *-algebra) is exponentiated, there is a natural compatibility between the continuous linear operators implementing the strongly continuous Lie group representation and the additional algebraic structure consisting of the (separately continuous) product (and the continuous involution, in the case of a $*$-algebra). This implies, therefore, that the Lie group representation is actually implemented by automorphisms (respectively, *-automorphisms) of the locally convex algebra (respectively, locally convex *-algebra) under consideration. This motivates a more convenient exponentiation definition in the realms of locally convex algebras and locally convex $*$-algebras, as is done in

\footnotetext{
${ }^{8}$ It was not known to the author, at the time when Corollary 3.4 was conceived, that this statement was already proved by S.J. Bhatt in [15, Proposition (3)]. However, the proof given here is more direct.
} 
Definition 3.6. A general characterization of the exponentiation issue in complete locally convex algebras and in complete locally convex *-algebras is fully obtained in Theorem 3.7, in the same spirit as in Theorem 2.14: (i) the higher-order operator dominating the Lie algebra representation is a general strongly elliptic operator in the (complexification of the) universal enveloping algebra; (ii) the dense, common and invariant core domain underlying the operator Lie algebra is the maximal $\mathrm{C}^{\infty}$ domain, and it will automatically be a subalgebra of the locally convex algebra in question - in the case of a locally convex *-algebra, such domain will be a *-subalgebra. Theorem 3.8 uses results of Ref. [26], from the $\mathrm{C}^{*}$-algebra context, to slightly strengthen the results of implication $(\Leftarrow)$ of Theorem 3.7 , in the case of locally $\mathrm{C}^{*}$-algebras, when the subjacent strongly elliptic operator is the negative of the (generalized) Laplacian. Finally, Theorem 3.9 gives a better adjusted version of Theorem 3.7 (in other words, with less hypotheses) in the case of locally $\mathrm{C}^{*}$ algebras, characterizing exponentiation when the strongly elliptic operator in question is the negative of the Laplacian. 


\section{Preliminaries}

A vector space which is also a topological space in a way that the operations of sum and product by scalars are continuous is called a topological vector space. When the origin of the topological vector space has a fundamental system of neighborhoods formed by convex sets, the space is called locally convex. These are the main types of topological vector spaces which will appear in this manuscript. ${ }^{1}$

If $\mathcal{X}$ is a vector space, then a seminorm $p$ on $\mathcal{X}$ is a function $p: \mathcal{X} \longrightarrow[0,+\infty)$ satisfying:

1. $p(x+y) \leq p(x)+p(y), \quad x, y \in \mathcal{X}$

2. $p(\lambda x)=|\lambda| p(x), \quad x \in \mathcal{X}, \lambda \in \mathbb{C}$.

If $\mathcal{X}$ is a topological vector space and $p$ is a continuous function on $\mathcal{X}$, then it is called a continuous seminorm. Local convexity is a concept closely related to that of a seminorm: it turns out, by a theorem, ${ }^{2}$ that the topology of every locally convex space can be generated by a family of seminorms or, more precisely, if $\mathcal{X}$ is such a space, then there exists a family $\left\{p_{\lambda}\right\}_{\lambda \in \Lambda}$ of seminorms defined on $\mathcal{X}$, called a fundamental system of seminorms for that topology, such that, for every neighborhood $U$ of the origin 0 of $\mathcal{X}$ and every $x \in U$ there exist an $\epsilon>0$ and a finite subset $F \subseteq \Lambda$ such that

$$
\bigcap_{\lambda \in F}\left\{y \in \mathcal{X}: p_{\lambda}(y-x)<\epsilon\right\} \subseteq U
$$

Equivalently, the family

$$
\left\{\bigcap_{\lambda \in F}\left\{y \in \mathcal{X}: p_{\lambda}(y-x)<\epsilon\right\}: x \in \mathcal{X}, \epsilon>0, F \subseteq \Lambda \text { is finite }\right\}
$$

\footnotetext{
${ }^{1}$ The vector spaces which appear in this thesis, with the exception of some Lie algebras, will always be vector spaces over $\mathbb{C}$. Also, some authors add the extra axiom on topological vector spaces or locally convex spaces as being, by definition, Hausdorff topological spaces - see [110, page 7] or [117, page 47], for example.

${ }^{2}$ See, for example, [121, page 63$]$.
} 
is a basis for the topology of $\mathcal{X}$. If $\mathcal{X} \neq\{0\}$, there is an infinite number of families of seminorms which generate its topology. Frequently, the notations $(\mathcal{X}, \Gamma)$ or $(\mathcal{X}, \tau)$ will be used to indicate, respectively, that $\Gamma$ is a fundamental system of seminorms for $\mathcal{X}$ or that $\tau$ is the locally convex topology of $\mathcal{X}$.

A useful fact is that if $(\mathcal{X}, \Gamma)$ is a locally convex space, then it is Hausdorff if, and only if,

$$
\bigcap_{\lambda \in \Lambda}\left\{x \in \mathcal{X}: p_{\lambda}(x)=0\right\}=\{0\} .
$$

It should be mentioned that the only locally convex spaces of interest in this manuscript are the Hausdorff ones. All inner product spaces and all normed spaces are locally convex spaces which are Hausdorff since they are, in particular, metric spaces.

A family of seminorms $\Gamma:=\left\{p_{\lambda}\right\}_{\lambda \in \Lambda}$ defined on a locally convex space $\mathcal{X}$ is said to be saturated if, for any given finite subset $F$ of $\Lambda$, the seminorm defined by

$$
p_{F}: x \longmapsto \max \{p(x): p \in F\}
$$

also belongs to $\Gamma$. Every fundamental system of seminorms can always be enlarged to a saturated one by including the seminorms $p_{F}$, as defined above, in such a way that the resulting family generates the same topology. Hence, it will always be assumed in this manuscript that the families of seminorms to be considered are already saturated, whenever convenient, without further notice.

A directed set $\mathcal{A}$ is a non-empty set with a partial order $\preceq$ such that, for all $\alpha_{1}, \alpha_{2} \in \mathcal{A}$, there exists another $\alpha_{3} \in \mathcal{A}$ satisfying $\alpha_{3} \succeq \alpha_{1}$ and $\alpha_{3} \succeq \alpha_{2}$. Using this concept, one may define a net in a locally convex space $\mathcal{X}$ as a function $x: \mathcal{A} \longrightarrow \mathcal{X}, \alpha \longmapsto x_{\alpha}$. Given a net $\left\{x_{\alpha}\right\}_{\alpha \in \mathcal{A}}$ in $\mathcal{X}$, it is said to converge to an element $x \in \mathcal{X}$ if, and only if,

$$
p\left(x_{\alpha}-x\right) \longrightarrow 0
$$

for all $p \in \Gamma$. A net $\left\{x_{\alpha}\right\}_{\alpha \in \mathcal{A}}$ in $\mathcal{X}$ is said to be Cauchy if for every neighborhood $U$ of 0 there exists $\alpha_{0} \in \mathcal{A}$ such that $x_{\alpha}-x_{\alpha^{\prime}} \in U$, if $\alpha, \alpha^{\prime} \succeq \alpha_{0}$. Equivalently, if $\Gamma$ is a fundamental system of seminorms for $\mathcal{X}$, then $\left\{x_{\alpha}\right\}_{\alpha \in \mathcal{A}}$ is a Cauchy net if, given $\epsilon>0$, for every $p \in \Gamma$ there exists $\alpha(p) \in \mathcal{A}$ such that $x_{\alpha}-x_{\alpha^{\prime}} \in U$, if $\alpha, \alpha^{\prime} \succeq \alpha(p)$. This is a necessary concept to define completeness: $\mathcal{X}$ is complete if every Cauchy net $\left\{x_{\alpha}\right\}_{\alpha \in \mathcal{A}}$ in $\mathcal{X}$ converges to an element $x \in \mathcal{X}$ (if $\mathcal{X}$ is Hausdorff, then this limit is unique). All Hilbert spaces and, more generally, all Banach spaces are complete locally convex spaces. A weaker kind of completeness is that of sequential completeness: if every Cauchy sequence $\left\{x_{n}\right\}_{n \in \mathbb{N}}$ in $\mathcal{X}$ converges to an element $x \in \mathcal{X}$, then $\mathcal{X}$ is called sequentially complete. A sequentially complete Hausdorff locally convex space whose underlying topology is defined by a countable fundamental system of seminorms is called a Fréchet space. Another kind 
of completeness, referred to as wafer completeness, is going to be introduced later in this chapter.

An important notion which is going to appear in this work is that of a bounded subset of $\mathcal{X}$. A subset $E \subseteq \mathcal{X}$ is said to be bounded if for every neighborhood $U$ of the origin there exists a $\lambda>0$ such that $E \subseteq \lambda U$. Equivalently, $E \subseteq \mathcal{X}$ is bounded if, and only if, for any given fundamental system of seminorms $\Gamma$ of $\mathcal{X}$, the restriction $\left.p\right|_{E}$ of every seminorm $p \in \Gamma$ is a bounded real-valued function.

Before passing to another subject, the concept of a barrelled space will be introduced. A barrel of $\mathcal{X}$ is a closed, convex, balanced and absorbing subset of $\mathcal{X}{ }^{3}$ If every barrel of $\mathcal{X}$ is a neighborhood of the origin, then $\mathcal{X}$ is said to be barrelled. ${ }^{4}$ The great importance of this kind of locally convex space resides in the fact that a generalization of the classical Uniform Boundedness Principle for Banach spaces is available for them - see [121, Theorem 33.1 , page 347].

\subsection{One-Parameter Semigroups and Groups}

In the classical theory of one-parameter semigroups and groups, a strongly continuous one-parameter semigroup on a normed $\operatorname{space}(\mathcal{Y},\|\cdot\|)$ is a family of continuous linear operators $\{V(t)\}_{t \geq 0}$ satisfying

$$
V(0)=I, V(s+t)=V(s) V(t), \quad s, t \geq 0
$$

and

$$
\lim _{t \rightarrow t_{0}}\left\|V(t) y-V\left(t_{0}\right) y\right\|=0, \quad t_{0} \geq 0, y \in \mathcal{Y} .
$$

It is a well-known fact that in this setting there exist $M>0$ and $a \geq 0$ satisfying

$$
\|V(t) y\| \leq M \exp (a t)\|y\|
$$

for all $y \in \mathcal{Y}$, as a consequence of the Uniform Boundedness Principle - see [50, Proposition 5.5]. If $a$ and $M$ can be chosen to be, respectively, 0 and 1, then it is called a contraction semigroup. All definitions are analogous for groups, switching from " $t \geq 0$ " to " $t \in \mathbb{R}$ " and $" \exp (a t) "$ to "exp $(a|t|) "$. Also, in the context of one-parameter groups, if $a$ and $M$ can be chosen to be, respectively, 0 and 1 , then it is called a group of isometries, or a

\footnotetext{
${ }^{3}$ A set $S \subseteq \mathcal{X}$ is balanced if $\mu S \subseteq S$ for all $\mu \in \mathbb{C}$ satisfying $|\mu| \leq 1$. It is called absorbing if, for every $x \in \mathcal{X}$, there exists $r_{x}>0$ such that $a x \in S$, for all $|a| \leq r_{x}$.

${ }^{4}$ The origin of every Hausdorff locally convex space has a fundamental system of neighborhoods constituted by barrels - see, for example, [121, Proposition 7.2, page 58]. However, in order for $\mathcal{X}$ to be barrelled, every barrel of $\mathcal{X}$ must be a neighborhood of the origin.
} 
representation by isometries.

The type of a strongly continuous semigroup $t \longmapsto V(t)$ on a Banach space $(\mathcal{Y},\|\cdot\|)$ is defined as the number

$$
\inf _{t>0} \frac{1}{t} \log \|V(t)\|
$$

- see [68, page 306]; see also [50, Definition 5.6, page 40]. Analogously, if $t \longmapsto V(t)$ is a strongly continuous group, its type is defined as

$$
\inf _{t \in \mathbb{R} \backslash\{0\}} \frac{1}{|t|} \log \|V(t)\| \text {. }
$$

The task, now, will be to formulate analogous concepts for locally convex spaces.

Definition (Equicontinuous Sets): Let $\mathcal{X}$ be a Hausdorff locally convex space with a fundamental system of seminorms $\Gamma$ and denote by $\mathcal{L}(\mathcal{X})$ the vector space of continuous linear operators defined on all of $\mathcal{X}$. A set $\Phi \subseteq \mathcal{L}(\mathcal{X})$ of linear operators is called equicontinuous if for every neighborhood $V$ of the origin of $\mathcal{X}$ there exists another neighborhood $U \subseteq \mathcal{X}$ of 0 such that $T[U] \subseteq V$, for every $T \in \Phi$ - equivalently, if for every $p \in \Gamma$ there exist $q \in \Gamma$ and $M_{p}>0$ satisfying

$$
p(T(x)) \leq M_{p} q(x)
$$

for all $T \in \Phi$ and $x \in \mathcal{X}$.

Definition (Equicontinuous and Exponentially Equicontinuous Semigroups and Groups): A one-parameter semigroup on a Hausdorff locally convex space $(\mathcal{X}, \Gamma)$ is a family of continuous linear operators $\{V(t)\}_{t \geq 0}$ satisfying

$$
V(0)=I, V(s+t)=V(s) V(t), \quad s, t \geq 0 .
$$

If, in addition, the semigroup satisfies the property that

$$
\lim _{t \rightarrow t_{0}} p\left(V(t) x-V\left(t_{0}\right) x\right)=0, \quad t_{0} \geq 0, p \in \Gamma, x \in \mathcal{X}
$$

then $\{V(t)\}_{t \geq 0}$ is called strongly continuous or, more explicitly, a strongly continuous one-parameter semigroup. Such a semigroup is called exponentially equicontinuous [3, Definition 2.1] if there exists $a \geq 0$ satisfying the following property: for all $p \in \Gamma$ there exist $q \in \Gamma$ and $M_{p}>0$ such that

$$
p(V(t) x) \leq M_{p} \exp (a t) q(x), \quad t \geq 0, x \in \mathcal{X}
$$

or, in other words, if the rescaled family

$$
\{\exp (-a t) V(t)\}_{t \geq 0} \subseteq \mathcal{L}(\mathcal{X})
$$


is equicontinuous [3, Definition 2.1]. If a can be chosen equal to 0, such a semigroup will be called equicontinuous. a strongly continuous semigroup $t \longmapsto V(t)$ is said to be locally equicontinuous if, for every compact $K \subseteq[0,+\infty)$, the set $\{V(t): t \in K\}$ is equicontinuous. (As was already mentioned before, every strongly continuous semigroup $V$ on a Banach space $\mathcal{Y}$ satisfies $\|V(t) y\| \leq M \exp ($ at $)\|y\|$, for all $t \geq 0$ and $y \in \mathcal{Y}$, so $V$ is automatically locally equicontinuous) All definitions are analogous for one-parameter groups, switching from " $t \geq 0$ " to " $t \in \mathbb{R}$ ", $" \exp ($ at $) "$ to $" \exp (a|t|) "$ and $[0,+\infty)$ to $\mathbb{R}$.

Unlike for semigroups on Banach spaces, a strongly continuous semigroup $t \longmapsto V(t)$ on a locally convex space $(\mathcal{X}, \Gamma)$ does not necessarily satisfy a global estimate analogous to (1.1.1) - see the third paragraph of [78, page 258]. However, if $\mathcal{X}$ is barrelled, this property holds at least locally, on compact subsets $K$ of $\mathbb{R}$. Namely, for all fixed $p \in \Gamma$ and $x \in \mathcal{X}$ the supremum

$$
\sup \{p(V(t) x): t \in K\}
$$

is finite, due to the continuity of $t \longmapsto V(t) x$. Therefore, by the Uniform Boundedness Principle for barrelled locally convex spaces, if $p \in \Gamma$ is a fixed seminorm, there exist $M_{p}>0$ and $q \in \Gamma$ such that

$$
p(V(t) x) \leq M_{p} q(x),
$$

for all $x \in \mathcal{X}$ and $t \in K$.

Note that, in the definition of exponentially equicontinuous semigroups, the seminorm $q$ does not need to be equal to $p$. This phenomenon motivates the definition of $\Gamma$-semigroups:

Definition $(\boldsymbol{\Gamma}$-semigroups and $\boldsymbol{\Gamma}$-groups): Let $(\mathcal{X}, \Gamma)$ be a Hausdorff locally convex space. For each $p \in \Gamma$, define

$$
V_{p}:=\{x \in \mathcal{X}: p(x) \leq 1\}
$$

Following [8], the following conventions will be used:

1. $\mathcal{L}_{\Gamma}(\mathcal{X})$ denotes the family of linear operators $T$ on $\mathcal{X}$ satisfying the property that, for all $p \in \Gamma$, there exists $\lambda(p, T)>0$ such that

$$
T\left[V_{p}\right] \subseteq \lambda(p, T) V_{p}
$$

or, equivalently,

$$
p(T(x)) \leq \lambda(p, T) p(x), \quad x \in \mathcal{X}
$$


2. A strongly continuous semigroup $t \longmapsto V(t)$ is said to be a $\boldsymbol{\Gamma}$-semigroup ${ }^{5}$ if $V(t) \in$ $\mathcal{L}_{\Gamma}(\mathcal{X})$, for all $t \geq 0$ and, for every $p \in \Gamma$ and $\delta>0$, there exists a number $\lambda=$ $\lambda(p,\{V(t): 0 \leq t \leq \delta\})>0$ such that

$$
p(V(t) x) \leq \lambda p(x),
$$

for all $0 \leq t \leq \delta$ and $x \in \mathcal{X}$. Analogously, a strongly continuous group $t \longmapsto V(t)$ is said to be a $\boldsymbol{\Gamma}$-group if $V(t) \in \mathcal{L}_{\Gamma}(\mathcal{X})$, for all $t \in \mathbb{R}$ and, for every $p \in \Gamma$ and $\delta>0$, there exists a number $\lambda=\lambda(p,\{V(t):|t| \leq \delta\})>0$ such that

$$
p(V(t) x) \leq \lambda p(x),
$$

for all $|t| \leq \delta$ and $x \in \mathcal{X}$ (see [8, Definitions 2.1 and 2.2] - note that a "local equicontinuity-type" requirement, which is present in Definition 2.1, is incorrectly missing in Definition 2.2). An equivalent way of defining then is the following: a strongly continuous one-parameter semigroup $t \longmapsto V(t)$ is said to be a $\boldsymbol{\Gamma}$-semigroup if, for each $p \in \Gamma$, there exist $M_{p}, \sigma_{p} \in \mathbb{R}$ such that $p(V(t) x) \leq M_{p} e^{\sigma_{p} t} p(x)$, for all $x \in \mathcal{X}$ and $t \geq 0$ - see [8, Theorem 2.6]. $\Gamma$-groups are defined in an analogous way, but with $p(V(t) x) \leq M_{p} e^{\sigma_{p}|t|} p(x)$, for all $x \in \mathcal{X}$ and $t \in \mathbb{R}$. Note that these definitions automatically imply local equicontinuity of the one-parameter (semi)group $V$, and that the operators $V(t)$ have the (KIP) with respect to $\Gamma$.

3. By a procedure which will become clearer in the future and which is closely related to some of the theory exposed in Section 1.4 (that section defines the (KIP) - Kernel Invariance Property - and explores some of its useful consequences), the author of [8] associates to the $\Gamma$-semigroup $t \longmapsto V(t)$ a net of strongly continuous semigroups $\left\{\tilde{V}_{p}\right\}_{p \in \Gamma}$, each one of them being defined on a Banach space $\left(\mathcal{X}_{p},\|\cdot\|_{p}\right)$. By what was already said above, for each $p \in \Gamma$, the number

$$
\inf _{t>0} \frac{1}{t} \log \left\|\tilde{V}_{p}(t)\right\|_{p}
$$

is well-defined, and will be denoted by $w_{p}$. The family $\left\{w_{p}\right\}_{p \in \Gamma}$ is called the type of $\boldsymbol{V}$. If $w:=\sup _{p \in \Gamma} w_{p}<\infty$, then $V$ is said to be of bounded type $\boldsymbol{w}$, following [8, page 170] - analogously, substituting " $t>0$ " by " $t \in \mathbb{R} \backslash\{0\}$ " and " $1 / t$ " by " $1 /|t|$ ", one defines $\Gamma$-groups of bounded type. A very nice aspect of $\Gamma$-semigroups of bounded type $w$ is that they satisfy the resolvent formula

$$
R(\lambda, A) x=\int_{0}^{+\infty} e^{-\lambda t} V(t) x d t, \quad x \in \mathcal{X}
$$

for all $\lambda>w$, where $A$ is the infinitesimal generator of $V$, as is shown in [8, Theorem 3.3] - for the definition of infinitesimal generator, see the next section. This formula

\footnotetext{
${ }^{5}$ In $[8]$ they are called $\left(\mathrm{C}_{0}, 1\right)$ semigroups - similarly for groups.
} 
will be the key for many proofs to come, like the ones of Lemma 1.2.4.3, Theorem 2.4 and Theorem 2.12.

Note that all of the definitions regarding semigroups and groups, above, with the exception of the last one (regarding $\Gamma$-semigroups and $\Gamma$-groups), are independent of the fixed fundamental system of seminorms $\Gamma$.

To conclude this section note that, in the group case, much of the above terminology can be adapted from one-parameter groups to general Lie groups. For example, if $G$ is a Lie group with unit $e$, then a family of continuous linear operators $\{V(g)\}_{g \in G}$ satisfying

$$
V(e)=I, V(g h)=V(g) V(h), \quad g, h \in G
$$

and

$$
\lim _{g \rightarrow h} V(g) x=V(h) x, \quad x \in \mathcal{X}, h \in G,
$$

is called a strongly continuous representation of $G$. Such a group representation is called locally equicontinuous if for each compact $K \subseteq G$ the set $\{V(g): g \in K\}$ is equicontinuous.

It should be emphasized, at this point, that the main object of study in this thesis is not the structure of locally convex spaces in itself, but rather families of linear operators defined on these spaces, like generators of one-parameter semigroups and groups of continuous operators and Lie algebras of linear operators, for example. These operators of interest are, in most cases, not assumed to be continuous, nor are they everywhere defined. Hence, if $\mathcal{X}$ is a locally convex space, a linear operator $T$ on $\mathcal{X}$ will always be assumed as being defined on a vector subspace Dom $T$ of $\mathcal{X}$, called its domain, and in case $\mathcal{X}$ is also an algebra (respectively, a $*$-algebra), Dom $T$ will, by definition, be a subalgebra (respectively, a $*$-subalgebra - in other words, a subalgebra which is closed under the operation $*$ of involution). When its domain is dense, the operator will be called densely defined. Also, if $S$ and $T$ are two linear operators then their sum will be defined by

$\operatorname{Dom}(S+T):=\operatorname{Dom} S \cap \operatorname{Dom} T, \quad(S+T)(x):=S(x)+T(x), \quad x \in \operatorname{Dom}(S+T)$ and their composition by

$$
\operatorname{Dom}(T S):=\{x \in \mathcal{X}: S(x) \in \operatorname{Dom} T\}, \quad(T S)(x):=T(S(x)), \quad x \in \operatorname{Dom}(T S),
$$

following the usual conventions of the classical theory of unbounded linear operators on Hilbert and Banach spaces. The range of $T$ will be denoted by Ran $T$

A very important concept is that of a resolvent set, so let $T:$ Dom $T \longrightarrow \mathcal{X}$ be a linear operator on $\mathcal{X}$. Then, the set

$$
\rho(T):=\left\{\lambda \in \mathbb{C}:(\lambda I-T): \text { Dom } T \subseteq \mathcal{X} \longrightarrow \mathcal{X} \text { is bijective and }(\lambda I-T)^{-1} \in \mathcal{L}(\mathcal{X})\right\}
$$


is called the resolvent set of the operator $T$ (note that it may happen that $\rho(T)=\emptyset$, or even $\rho(T)=\mathbb{C})$, and the map

$$
\mathrm{R}(\cdot, T): \rho(T) \ni \lambda \longmapsto \mathrm{R}(\lambda, T):=(\lambda I-T)^{-1} \in \mathcal{L}(\mathcal{X})
$$

is called the resolvent of $\boldsymbol{T}$. When $\lambda \in \rho(T)$, it will be said that the resolvent operator of $\boldsymbol{T}$ exists at $\boldsymbol{\lambda}$, and $\mathrm{R}(\lambda, T)$ is called the resolvent operator of $\boldsymbol{T}$ at $\boldsymbol{\lambda}$ or the $\boldsymbol{\lambda}$ resolvent of $T$. Finally, the complementary set

$$
\sigma(T):=\mathbb{C} \backslash \rho(T)
$$

is called the spectrum of the operator $T$ (see page 258 of [2]).

Now, a central notion for this work is going to be defined: that of an infinitesimal generator.

\subsection{Lie Group Representations and Infinitesimal Generators}

Definition (Infinitesimal Generators): Given a strongly continuous one-parameter semigroup $t \longmapsto V(t)$ on $(\mathcal{X}, \Gamma)$, consider the subspace of vectors $x \in \mathcal{X}$ such that the limit

$$
\lim _{t \rightarrow 0} \frac{V(t) x-x}{t}
$$

exists. Then, the linear operator $A$ defined by

$$
\operatorname{Dom} A:=\left\{x \in \mathcal{X}: \lim _{t \rightarrow 0} \frac{V(t) x-x}{t} \text { exists in } \mathcal{X}\right\}
$$

and $A(x):=\lim _{t \rightarrow 0} \frac{V(t) x-x}{t}$ is called the infinitesimal generator (or, simply, the generator) of the semigroup $t \longmapsto V(t)$ (the definition for groups is analogous). Also, if $G$ is a Lie group then, for each fixed element $X$ of its Lie algebra $\mathfrak{g}$, the infinitesimal generator of the one-parameter group $t \longmapsto V(\exp t X)$ will be denoted by $d V(X)$ (exp denotes the exponential map of the Lie group $G$ ).

Two very important results regarding infinitesimal generators on locally convex spaces are Propositions 1.3 and 1.4 of [78], which prove that infinitesimal generators of strongly continuous locally equicontinuous one-parameter semigroups on sequentially complete locally convex spaces are densely defined and closed. ${ }^{6}$

\footnotetext{
${ }^{6} \mathrm{~A}$ linear operator $T:$ Dom $\mathrm{T} \subseteq \mathcal{X} \longrightarrow \mathcal{X}$ is closed if its graph is a closed subspace of $\mathcal{X} \times \mathcal{X}$. An operator $S:$ Dom $\mathrm{S} \subseteq \mathcal{X} \longrightarrow \mathcal{X}$ is closable if it has a closed extension or, equivalently, if for every net $\left\{x_{\alpha}\right\}_{\alpha \in \mathcal{A}}$ in Dom $S$ such that $x_{\alpha} \longrightarrow 0$ and $S\left(x_{\alpha}\right) \longrightarrow y$, one has $y=0$. If $S$ is closable, then it has a minimal closed extension, called the closure of $S$ and denoted by $\bar{S}$ - see [104, page 250].
} 
A closable linear operator with the property that its closure is an infinitesimal generator is called an infinitesimal pregenerator.

Before proceeding to general strongly continuous Lie group representations, a few words about generators of strongly continuous one-parameter semigroups and groups on Hilbert and Banach spaces will be in order.

A strongly continuous one-parameter group $t \longmapsto V(t)$ on a Banach space gives rise to two strongly continuous semigroups $V_{+}$and $V_{-}$defined by $V_{+}(t):=V(t)$ and $V_{-}(t):=$ $V(-t)$, for all $t \geq 0$. It is clear by their definitions that if $A$ denotes the infinitesimal generator of $V$, then the generators of $V_{+}$and $V_{-}$are, respectively, $A$ and $-A$.

In the classical theory of one-parameter semigroups and groups in Hilbert and Banach spaces there exist some illustrious theorems which characterize their infinitesimal generators (or pregenerators, sometimes). In the Hilbert space context, the Spectral Theorem for self-adjoint operators - more precisely, the functional calculus for self-adjoint operators together with Stone's Theorem characterizes not only which are the strongly continuous one-parameter groups by unitary operators but also says that their generators are precisely the anti-adjoint operators acting on the space. ${ }^{7}$ In the Banach spaces setting, characterizations of the generators of strongly continuous semigroups are given by two very well-known theorems. Some authors refer to both theorems below as Hille-Yosida Theorems, but some attribute this name only to the first one, while the last is called the Feller-Miyadera-Phillips Theorem:

Hille-Yosida Theorem [50, Theorem 3.5, page 73]: For a linear operator $A$ on a Banach space $\mathcal{Y}$ the following properties are equivalent:

1. A generates a strongly continuous contraction semigroup.

2. A is closed, densely defined and for every $\lambda>0$ one has $\lambda \in \rho(A)$ and

$$
\|\lambda R(\lambda, A)\| \leq 1
$$

3. A is closed, densely defined and for every $\lambda \in \mathbb{C}$ with $\operatorname{Re} \lambda>0$ one has $\lambda \in \rho(A)$ and

$$
\|R(\lambda, A)\| \leq \frac{1}{R e \lambda}
$$

Feller-Miyadera-Phillips Theorem [50, Theorem 3.8, page 77]: Let $A$ be a linear operator on a Banach space $\mathcal{Y}$ and let $w \in \mathbb{R}, M \geq 1$ be constants. Then, the following properties are equivalent:

\footnotetext{
${ }^{7}$ For a proof of Stone's Theorem see [104, Theorem VIII.8, page 266]. See also [32].
} 
1. A generates a strongly continuous semigroup $\{V(t)\}_{t \geq 0}$ satisfying

$$
\|V(t)\| \leq M \exp (w t), \quad t \geq 0 .
$$

2. A is closed, densely defined and for every $\lambda>w$ one has $\lambda \in \rho(A)$ and

$$
\left\|[(\lambda-w) R(\lambda, A)]^{n}\right\| \leq M, \quad n \in \mathbb{N} .
$$

3. A is closed, densely defined and for every $\lambda \in \mathbb{C}$ with Re $\lambda>w$ one has $\lambda \in \rho(A)$ and

$$
\left\|R(\lambda, A)^{n}\right\| \leq \frac{M}{(\operatorname{Re} \lambda-w)^{n}}, \quad n \in \mathbb{N} .
$$

Substituting "strongly continuous contraction semigroup" by "strongly continuous group of isometries", $\lambda>0$ by $\lambda \in \mathbb{R} \backslash\{0\}$, Re $\lambda>0$ by $\mathbb{C} \backslash i \mathbb{R}$ and $\operatorname{Re} \lambda$ by $|\operatorname{Re} \lambda|$ in the first theorem gives a characterization for generators of groups of isometries. The second one also has a "group version", with minor changes in the statement: $\exp (a t) \rightarrow \exp (a|t|)$, $\lambda>w \rightarrow|\lambda|>w,[(\lambda-w) \mathrm{R}(\lambda, A)]^{n} \rightarrow[(|\lambda|-w) \mathrm{R}(\lambda, A)]^{n}, \operatorname{Re} \lambda>w \rightarrow|\operatorname{Re} \lambda|>w$ and $(\operatorname{Re} \lambda-w)^{n} \rightarrow(|\operatorname{Re} \lambda|-w)^{n}$. This can be seen in [50, Corollary 3.7, page 76] and [50, 3.8 Generation Theorem for Groups, page 79], which is reproduced here, for the convenience of the reader:

Feller-Miyadera-Phillips Theorem for One-Parameter Groups [50, page 79]: Let $A$ be a linear operator on a Banach space $\mathcal{Y}$ and let $w \in \mathbb{R}, M \geq 1$ be constants. Then, the following properties are equivalent:

1. A generates a strongly continuous group $\{V(t)\}_{t \in \mathbb{R}}$ satisfying

$$
\|V(t)\| \leq M \exp (w|t|), \quad t \in \mathbb{R} .
$$

2. $A$ and $-A$ are the generators of strongly continuous semigroups $V_{+}$and $V_{-}$, respectively, which satisfy

$$
\left\|V_{+}(t)\right\|,\left\|V_{-}(t)\right\| \leq M \exp (w|t|), \quad t \in \mathbb{R} .
$$

3. $A$ is closed, densely defined and for every $\lambda \in \mathbb{R}$ with $|\lambda|>w$ one has $\lambda \in \rho(A)$ and

$$
\left\|[(|\lambda|-w) R(\lambda, A)]^{n}\right\| \leq M, \quad n \in \mathbb{N} .
$$

4. $A$ is closed, densely defined and for every $\lambda \in \mathbb{C}$ with $\mid$ Re $\lambda \mid>$ w one has $\lambda \in \rho(A)$ and

$$
\left\|R(\lambda, A)^{n}\right\| \leq \frac{M}{(|\operatorname{Re} \lambda|-w)^{n}}, \quad n \in \mathbb{N} .
$$


Still in the Banach space setting, there are at least two more important theorems regarding the characterization of generators, but only one of them will be mentioned for now - the other one will appear in Section 1.4, when the definition of projective analytic vectors will be introduced. But first, a definition is needed. Let $(\mathcal{Y},\|\cdot\|)$ be a Banach space. A linear operator $T: \operatorname{Dom} T \subseteq \mathcal{Y} \longrightarrow \mathcal{Y}$ is called dissipative if for every $\mu>0$ and $y \in \operatorname{Dom} T$,

$$
\|(\mu I-T) y\| \geq \mu\|y\| .
$$

In particular, $\mu I-T$ is an injective linear operator, for every $\mu>0$. If both $T$ and $-T$ are $\Gamma$-dissipative, $T$ is said to be conservative, so that every conservative operator is dissipative. The following theorem is known as the Lumer-Phillips Theorem:

Lumer-Phillips Theorem [50, Theorem 3.15, page 83]: For a densely defined, dissipative operator on a Banach space $\mathcal{Y}$ the following statements are equivalent:

1. The closure $\bar{A}$ of $A$ generates a contraction semigroup.

2. Ran $(\lambda-A)$ is dense in $\mathcal{Y}$ for some (and hence all) $\lambda>0$.

Again, this theorem also has a version for groups of isometries, substituting $\lambda>0$ by $\lambda \in \mathbb{R} \backslash\{0\}$ and the word "dissipative" by the word "conservative".

The three theorems mentioned above have more general versions valid for locally convex spaces, which will be discussed in Section 1.4.

Definition (Smooth and Analytic Vectors): Returning to the original subject of this section, if $\mathcal{X}$ is a Hausdorff locally convex space and $V: G \longrightarrow \mathcal{L}(\mathcal{X})$ is a strongly continuous representation then a vector $x \in \mathcal{X}$ is called a $C^{\infty}$ vector for $\boldsymbol{V}$, or $\boldsymbol{a}$ smooth vector for $\boldsymbol{V}$, if the map $G \ni g \longmapsto V(g) x$ is of class $C^{\infty}:$ a map $f: G \longrightarrow \mathcal{X}$ is of class $C^{\infty}$ at $g \in G$ if it possesses continuous partial derivatives of all orders with respect to a chart around $g$. If $f$ is of class $C^{\infty}$ at all points $g \in G, f$ is said to be of class $C^{\infty}$ on $G .^{8}$ The subspace of smooth vectors for $V$ will be denoted by $C^{\infty}(V)$. Moreover, following [91, page 54], a vector $x \in \mathcal{X}$ is called analytic for $\boldsymbol{V}$ if $x \in C^{\infty}(V)$ and the map $F_{x}: G \ni g \longmapsto V(g) x$ is analytic: in other words, if $x \in C^{\infty}(V)$ and, for each $g \in G$ and every analytic chart $h: g^{\prime} \longrightarrow\left(t_{k}\left(g^{\prime}\right)\right)_{1 \leq k \leq d}$ around $g$ sending it to 0 there exists $r_{x}>0$ such that the series

$$
\sum_{\alpha \in \mathbb{N}^{d}} \frac{p\left(\partial^{\alpha} F_{x}\left(g^{\prime}\right)\right)}{\alpha !} t\left(g^{\prime}\right)^{\alpha}
$$

\footnotetext{
${ }^{8}$ This definition of a smooth vector may be found in [91, page 47]. It should be mentioned, however, that defining a smooth vector $x$ by asking that $G \ni g \longmapsto V(g) x$ must be a smooth map with respect to the weak topology, instead, would be "more natural", in a certain sense: the verification of chart independence of this definition, for example, amounts to the usual proof one finds in finite dimensional Differential Geometry. In a wafer complete Hausdorff locally convex space - this definition will be introduced, soon - these two notions of smoothness coincide, as it is shown in [91, Lemma 1, page 47].
} 
is absolutely convergent to $F_{x}\left(g^{\prime}\right)$, for every $p \in \Gamma$, whenever $\left|t\left(g^{\prime}\right)\right|<r_{x}$, where $t\left(g^{\prime}\right)^{\alpha}=$ $t_{1}\left(g^{\prime}\right)^{\alpha_{1}} \ldots t_{d}\left(g^{\prime}\right)^{\alpha_{d}}$ and $\left|t\left(g^{\prime}\right)\right|:=\max _{1 \leq k \leq d}\left|t_{k}\left(g^{\prime}\right)\right|$ (note that $r_{x}$ is independent of $\left.p \in \Gamma\right) .^{9}$ The subspace of analytic vectors for $V$ will be denoted by $C^{\omega}(V)$. If $\tau$ is the topology defined by $\Gamma$, then the elements of $C^{\omega}(V)$ will sometimes be called $\boldsymbol{\tau}$-analytic. Also, if the Lie group under consideration is $\mathbb{R}$, then the subspace of analytic vectors will be denoted by $C^{\omega}(T)$, where $T$ is the infinitesimal generator of $V$, and called the the subspace of analytic vectors for $T$.

An important observation is that, if

$$
f: g \longmapsto V(g) x
$$

is of class $\mathrm{C}^{\infty}$ at $e$, then it is of class $\mathrm{C}^{\infty}$ on all of $G$. In fact, fixing $\sigma \in G$ and defining the function $\phi_{\sigma}: G \ni g \longmapsto \sigma^{-1} g \in G$, then

$$
\left.f(g)=V(\sigma)\left[\left(f \circ \phi_{\sigma}\right)(g)\right)\right],
$$

for every $g \in G$. Since $V(\sigma)$ is a continuous linear operator on $\mathcal{X}, \phi_{\sigma}$ is of class $\mathrm{C}^{\infty}$ at $\sigma$ and $f$ is of class $\mathrm{C}^{\infty}$ at $e$, it follows that $f$ is of class $\mathrm{C}^{\infty}$ at $\sigma$. Hence, in order to verify that a vector $x$ is smooth for the representation $V$ it suffices to show that $g \longmapsto V(g) x$ is of class $\mathrm{C}^{\infty}$ at $e$. An analogous observation is valid for analytic vectors.

From now, until the end of Section 1.2, consider a fixed strongly continuous representation $V$ of the Lie group $G$, with Lie algebra $\mathfrak{g}$, on the Hausdorff locally convex space $\mathcal{X}$.

\subsubsection{The Gårding Subspace}

Fix a left invariant Haar measure on $G$. There exists an important subspace of $C^{\infty}(V)$ defined by

$$
D_{G}(V):=\operatorname{span}_{\mathbb{C}}\left\{x_{V}(\phi), \phi \in C_{c}^{\infty}(G), x \in \mathcal{X}\right\}, \quad \text { where } \quad x_{V}(\phi):=\int_{G} \phi(g) V(g) x d g,
$$

known as the Gårding subspace of $V$ - see [57]. When there is no danger of confusion, the subscript " $V$ " will be suppressed, so a generic element of $D_{G}(V)$ will often read as " $x(\phi)$ ". To begin with, the meaning of the integrals defining these elements must be specified. This can be accomplished by appropriately adapting a beautiful argument originally formulated within a Banach space context in $\left[7\right.$, page 219]. ${ }^{10}$ But before proceeding to the details, it should be emphasized that one additional hypothesis will be needed: $\mathcal{X}$ is going to

\footnotetext{
${ }^{9}$ Note, also, that [91, Lemma 3, page 52] proves that the convergence mentioned holds in any Hausdorff locally convex topology between the weak and the strong ones, and with the same radius of convergence.

${ }^{10}$ This argument remains valid if $G$ is only a Hausdorff locally compact topological group.
} 
be assumed complete. Actually, the property really needed here is that $\mathcal{X}$ be a wafer complete locally convex space or, in other words, a space with the property that the weakly closed convex balanced hull ${ }^{11}$ of every weakly compact set is weakly compact. A weakly closed set is a closed set of $\mathcal{X}$ when the latter is equipped with the topology induced by the family $\left\{x \longmapsto|f(x)|: f \in \mathcal{X}^{\prime}, x \in \mathcal{X}\right\}$ of seminorms; similarly, a weakly compact set is a compact set of $\mathcal{X}$ when the latter is equipped with this same topology. When $\mathcal{X}$ is considered equipped with this Hausdorff locally convex topology, called the weak topology on $\mathcal{X}$, it will be written $\mathcal{X}_{\sigma}$. Fortunately, by [91, Corollary 8, page 19], every complete locally convex space is wafer complete, so the hypothesis of completeness assumed here is sufficient to define the elements of $D_{G}(V)$. Since being complete will also be a necessary requirement at many later points of this work, it will be convenient to assume this stronger hypothesis on the underlying locally convex space right from the start, instead of merely supposing it to be wafer complete. This concept of wafer completeness is also going to appear in another situation, very soon.

So let $\Psi: G \ni g \longmapsto \Psi(g) \in \mathcal{X}_{\sigma}$ be a compactly supported continuous function, so that it is continuous with respect to the topology on $G$ and the weak topology on $\mathcal{X}$. By definition, the integral $\int_{G} \Psi(g) d g$ exists in $\mathcal{X}$ if there exists an element $y_{\Psi} \in \mathcal{X}$ such that, for all $f \in \mathcal{X}^{\prime}$,

$$
f\left(y_{\Psi}\right)=\int_{G} f(\Psi(g)) d g .{ }^{12}
$$

What is going to be shown is that, under these circumstances, existence of the integral $\int_{G} \Psi(g) d g$ in this sense is guaranteed. Indeed, fix a fundamental system of seminorms $\Gamma$ for $\mathcal{X}$ and define the linear functional $\eta$ on $\mathcal{X}^{\prime}$ by

$$
\eta(f):=\int_{G} f(\Psi(g)) d g, \quad f \in \mathcal{X}^{\prime} .
$$

Then, the claim is that there exists $y_{\Psi} \in \mathcal{X}$ such that $\eta(f)=f\left(y_{\Psi}\right)$, for all $f \in \mathcal{X}^{\prime}$. One has

$$
|\eta(f)| \leq \operatorname{vol}(\operatorname{supp} \Psi)\left[\sup _{g \in \operatorname{supp} \Psi}|f(\Psi(g))|\right]<, \quad f \in \mathcal{X}^{\prime},
$$

where $\operatorname{vol}(\operatorname{supp} \Psi)$ is the volume of the support of $\Psi$ with respect to the fixed Haar measure of $G$ (in other words, it is the evaluation of the measure on the compact set supp $\Psi$ - a Haar measure on $G$ is finite on compact subsets of $G$ [64]) and finiteness of the "sup" is a consequence of the continuity of $f \circ \Psi-f \circ \Psi$ is continuous because of the weak continuity of $\Psi$. When $\left(\mathcal{X}^{\prime}, \mathcal{X}\right)$ is considered as a dual pair, a dual topology on $\mathcal{X}^{\prime}$ may be defined by $\mathcal{X}$ via the family $\{f \longmapsto|f(x)|: x \in \mathcal{X}\}$ of seminorms. When $\mathcal{X}^{\prime}$ is equipped with this Hausdorff locally convex topology - usually referred to as the weak topology on $\mathcal{X}$ ' or the

\footnotetext{
${ }^{11}$ The balanced hull of a set $S \subseteq \mathcal{X}$ is the smallest balanced set which contains $S$. The weakly closed convex balanced hull of a set $S \subseteq \mathcal{X}$ is the smallest weakly closed convex balanced set which contains $S$.

${ }^{12}$ See, also, [74], page 443.
} 
topology of pointwise convergence, it is going to be denoted by $\mathcal{X}_{\sigma}^{\prime}$. If one uses the axioms of dual topologies it is not hard to prove that $\left(\mathcal{X}_{\sigma}^{\prime}\right)^{\prime}=\left\{f \longmapsto f(x), x \in \mathcal{X}, f \in \mathcal{X}^{\prime}\right\}$ (see [72, Theorem 2, page 147], for example), so proving the claim about $\eta$ is equivalent to proving that $\eta \in\left(\mathcal{X}_{\sigma}^{\prime}\right)^{\prime}$. But by the Mackey-Arens Theorem, if $\mathcal{X}^{\prime}$ is equipped with the Mackey topology which is, by definition, the Hausdorff locally convex topology induced by the family

$$
\left\{f \longmapsto \sup _{x \in L}|f(x)|: L \text { is a convex, balanced, weakly compact subset of } \mathcal{X}\right\}
$$

of seminorms (in this case, it is going to be denoted by $\left.\mathcal{X}_{\tau}^{\prime}\right)$, then $\left(\mathcal{X}_{\sigma}^{\prime}\right)^{\prime}=\left(\mathcal{X}_{\tau}^{\prime}\right)^{\prime}-^{13}$ this situation is usually described by saying that the Mackey topology on $\mathcal{X}^{\prime}$ is compatible with duality between $\mathcal{X}_{\sigma}^{\prime}$ and $\left(\mathcal{X}_{\sigma}^{\prime}\right)^{\prime}$. Therefore, it is sufficient to find a convex, balanced, weakly compact subset $L$ of $\mathcal{X}$ such that

$$
|\eta(f)| \leq C \sup _{x \in L}|f(x)|, \quad f \in \mathcal{X}^{\prime},
$$

for some $C>0$. This will establish that $\eta \in\left(\mathcal{X}_{\tau}^{\prime}\right)^{\prime}=\left(\mathcal{X}_{\sigma}^{\prime}\right)^{\prime}$, giving the desired result.

The set $\{\Psi(g): g \in \operatorname{supp} \Psi\}$ is weakly compact, since $\Psi$ is weakly continuous and the support of $\Psi$ is compact. Define $K:=\{\lambda \cdot \Psi(g): g \in \operatorname{supp} \Psi, \lambda \in \mathbb{C},|\lambda| \leq 1\}$. Then, $K$ is weakly compact, due to compactness of $\{\lambda \in \mathbb{C}:|\lambda| \leq 1\} \times \operatorname{supp} \Psi$ and continuity of the operation of product by scalars. Therefore, by the hypothesis of completeness assumed, the weakly closed convex hull $L$ of $K$ is weakly compact. It is also balanced, since the closure of a balanced set is balanced, and it contains $\Psi[\operatorname{supp} \Psi]$. Hence,

$$
|\eta(f)| \leq \operatorname{vol}(\operatorname{supp} \Psi)\left[\sup _{g \in \operatorname{supp} \Psi}|f(\Psi(g))|\right] \leq \operatorname{vol}(\operatorname{supp} \Psi) \sup _{x \in L}|f(x)|, \quad f \in \mathcal{X}^{\prime} .
$$

Finally, this shows that there exists an element $y_{\Psi} \in \mathcal{X}$ such that

$$
f\left(y_{\Psi}\right)=\int_{G} f(\Psi(g)) d g, \quad f \in \mathcal{X}^{\prime} .
$$

A very useful consequence of this fact is that the same element $y_{\Psi} \in \mathcal{X}$ also satisfies

$$
T\left(y_{\Psi}\right)=\int_{G} T(\Psi(g)) d g, \quad T \in \mathcal{L}(\mathcal{X}),
$$

since $f \circ T \in \mathcal{X}^{\prime}$, for all $f \in \mathcal{X}^{\prime}$ and $T \in \mathcal{L}(\mathcal{X})$.

Substituting $\Psi(g)$ by $\phi(g) V(g) x$, with $g \in G, \phi \in C_{c}^{\infty}(G)$ and $x \in \mathcal{X}$, this proves, in particular, that $x_{V}(\phi):=\int_{G} \phi(g) V(g) x d g$ is a well-defined element of $\mathcal{X}$, as desired. Again,

$$
T\left(x_{V}(\phi)\right)=\int_{G} \phi(g) T(V(g) x) d g, \quad x_{V}(\phi) \in D_{G}(V), T \in \mathcal{L}(\mathcal{X}) .
$$

\footnotetext{
${ }^{13}$ Note that $\sup _{x \in L}|f(x)|<\infty$, because $\left.f\right|_{L}$ is a weakly continuous function on a weakly compact set.
} 
Also, some immediate estimates may be derived:

$$
|f(x(\phi))| \leq \operatorname{vol}(\operatorname{supp} \phi)\left[\sup _{g \in \operatorname{supp} \phi}|\phi(g)|\right]\left[\sup _{g \in \operatorname{supp} \phi}|f(V(g) x)|\right], \quad f \in \mathcal{X}^{\prime} .
$$

Now since, according to a corollary of the Hahn-Banach Theorem,

$$
\sup _{f \in V_{p}^{\circ}}|f(y)|=p(y),{ }^{14}
$$

for all $y \in \mathcal{X}$ (remember $V_{p}:=\{x \in \mathcal{X}: p(x) \leq 1\}$ ), this gives

$$
p(x(\phi)) \leq \operatorname{vol}(\operatorname{supp} \phi)\left[\sup _{g \in \operatorname{supp} \phi}|\phi(g)|\right]\left[\sup _{g \in \operatorname{supp} \phi} p(V(g) x)\right], \quad p \in \Gamma .
$$

$D_{G}(V)$ is indeed a subspace of $C^{\infty}(V),{ }^{15}$ as can be seen by an argument adapted from [57]: if $x_{V}(\phi) \in D_{G}(V), X \in \mathfrak{g}$ and $t \neq 0$, then

$$
\begin{gathered}
\frac{V(\exp t X)\left(x_{V}(\phi)\right)-x_{V}(\phi)}{t}=\int_{G} \phi(g)\left[\frac{V([\exp t X] g)-V(g)}{t}\right] x d g \\
=\int_{G} \frac{\phi([\exp -t X] g)-\phi(g)}{t} V(g) x d g
\end{gathered}
$$

by the left invariance of the Haar measure. Hence, the Dominated Convergence Theorem together with the Hahn-Banach Theorem gives

$$
\lim _{t \rightarrow 0} \frac{V(\exp t X)\left(x_{V}(\phi)\right)-x_{V}(\phi)}{t}=\int_{G} \lim _{t \rightarrow 0} \frac{\phi([\exp -t X] g)-\phi(g)}{t} V(g) x d g,
$$

showing that $x_{V}(\phi) \in \operatorname{Dom} d V(X)$ and that

$$
d V(X)\left(x_{V}(\phi)\right)=-x_{V}\left(\tilde{X}^{R}(\phi)\right),
$$

\footnotetext{
${ }^{14}$ The quick proof of this fact which will be given here is taken from the answer of Davide Giraudo at topic "On polar sets with respect to continuous seminorms", on math.stackexchange.com: fix $x \in \mathcal{X}, p \in \Gamma$ and define the polar set of $V_{p}$ by $V_{p}^{\circ}:=\left\{f \in \mathcal{X}^{\prime}:|f(x)| \leq 1\right.$, for all $\left.x \in V_{p}\right\}$. If $p(x)=0$, then $r x \in V_{p}$ for all $r>0$, so $|f(x)| \leq(1 / r)$ for all $r>0$ and $f \in V_{p}^{\circ}$. Therefore, $\sup _{f \in V_{p}^{\circ}}|f(x)|=0=p(x)$. Now if $p(x) \neq 0$, on one side $x / p(x) \in V_{p}$, so $|f(x)| \leq p(x)$ for all $f \in V_{p}^{\circ}$, giving $\sup _{f \in V_{p}^{\circ}}|f(x)| \leq p(x)$. On the other hand, the continuous linear functional $f_{0}$ on $\mathbb{C} x$ defined by $f_{0}: a x \longmapsto a p(x)$ can by the Hahn-Banach Theorem be extended to a continuous linear functional $\tilde{f}_{0}$ on $\mathcal{X}$ which satisfies $\tilde{f}_{0}(x)=p(x)$ and $\left|\tilde{f}_{0}(y)\right| \leq p(y)$, for all $y \in \mathcal{X}$, so that $\tilde{f}_{0} \in V_{p}^{\circ}$ and $\sup _{f \in V_{p}^{\circ}}|f(x)| \geq p(x)$, finishing the proof.

${ }^{15}$ If $\mathcal{X}$ is a Fréchet space, then the Gårding subspace actually coincides with the space of smooth vectors - see [35] or [45].
} 
where $\tilde{X}^{R}$ is the right invariant vector field on $G$ corresponding to $X$. An iteration of this argument shows that $D_{G}(V) \subseteq C^{\infty}(V)$. Moreover, fix a sequence $\left\{\phi_{n}\right\}_{n \in \mathbb{N}}$ of nonnegative functions in $C_{c}^{\infty}(G)$ with

$$
\int_{G} \phi_{n} d g=1, \quad n \in \mathbb{N}
$$

and with the property that for every open neighborhood $U$ of the origin there exists $n_{U} \in \mathbb{N}$ such that supp $\phi_{n} \subseteq U$ for all $n \geq n_{U}$. Then, $x_{V}\left(\phi_{n}\right) \longrightarrow x$ in $\mathcal{X}$, when $n \longrightarrow+\infty$, since $V$ is strongly continuous. This establishes that $D_{G}(V)$ and hence also $C^{\infty}(V)$ is dense in $\mathcal{X}$. Note that the above results regarding $D_{G}(V)$ remain valid if instead of a left invariant Haar measure one had chosen a right invariant one (and vice-versa). This is true due to the facts that the modular function $g \longmapsto \Delta(g)$ is smooth (use Proposition 8.27 of [77] together with the fact that every point of $G$ is contained in a connected open set, by the definition of chart) and because integration with respect to a left invariant (respectively, right invariant) measure leads to integration with respect to a right invariant (respectively, left invariant) measure via a change of variables involving the modular function and the operation of inversion (see Corollary 8.30 of [77]): for example, if $d g$ is a left invariant Haar measure, then $\Delta(g) d g$ is a right invariant Haar measure and

$$
\int_{G} \psi\left(g^{-1}\right) d g=\int_{G} \psi(g) \Delta(g) d g, \quad \psi \in C_{c}^{\infty}(G)
$$

Conversely, if $d g$ is a right invariant Haar measure, then $\Delta(g)^{-1} d g$ is a left invariant Haar measure and

$$
\int_{G} \psi\left(g^{-1}\right) d g=\int_{G} \psi(g) \Delta(g)^{-1} d g, \quad \psi \in C_{c}^{\infty}(G) .
$$

\subsubsection{The Space of Smooth Vectors is Left Invariant by the Generators}

Suppose that $\mathcal{X}$ is a wafer complete Hausdorff locally convex space. Fix $x \in C^{\infty}(V)$, $h: G \supseteq V \longrightarrow W \subseteq \mathbb{R}^{d}$ a chart of $G$ around $e$ and define the function

$$
f: g \longmapsto V(g) x .
$$

If $X$ belongs to the Lie algebra of $G$, denoted by $\mathfrak{g}$, then $c: t \longmapsto \exp t X$ is an infinitely differentiable curve in $G$ which has $X$ as its tangent vector at $t=0$. As $f \circ c=\left(f \circ h^{-1}\right) \circ$ $(h \circ c)$, the limit

$$
\lim _{t \rightarrow 0} \frac{V(\exp t X) x-x}{t}
$$

exists, by the chain rule for directional derivatives on locally convex spaces - see Lemma 1.4 of [60], for example. Therefore, for each fixed $X \in \mathfrak{g}$, the linear operator $d V(X): C^{\infty}(V) \longrightarrow$ $\mathcal{X}$

$$
d V(X): x \longmapsto \lim _{t \rightarrow 0} \frac{V(\exp t X) x-x}{t}
$$


is well-defined. Besides, $C^{\infty}(V)$ is left invariant by $d V(X)$ : fix an ordered basis $\left(X_{k}\right)_{1 \leq k \leq d}$ of $\mathfrak{g}$. Then, it is clear that

$$
\mathcal{D}_{\infty}:=\bigcap_{k=1}^{d} \bigcap_{n=1}^{+\infty} \operatorname{Dom} d V\left(X_{k}\right)^{n} \supseteq C^{\infty}(V) .
$$

In order to prove the reverse inclusion, an adapted argument of Theorem 1.1 of [58] can be made: fix $x \in \mathcal{D}_{\infty}, f \in \mathcal{X}^{\prime}$, a right invariant Haar measure $d g$ on $G$ and a $\phi \in C_{c}^{\infty}(G)$. Then, denoting by $\tilde{X}_{k}$ the complete, globally defined and left invariant vector field on $G$ such that $\tilde{X}_{k}(e)=X_{k}$, for all $1 \leq k \leq d,{ }^{16}$ one obtains

$$
\begin{gathered}
\int_{G} \phi(g) f\left(V(g) d V\left(X_{k}\right)^{n}(x)\right) d g=\left.\int_{G} \frac{d^{n}}{d t^{n}}\left[\phi(g) f\left(V\left(g \exp t X_{k}\right) x\right)\right]\right|_{t=0} d g \\
=\left.\int_{G} \frac{d^{n}}{d t^{n}}\left[\phi\left(g \exp -t X_{k}\right) f(V(g) x)\right]\right|_{t=0} d g=(-1)^{n} \int_{G} \tilde{X}_{k}^{n}(\phi)(g) f(V(g) x) d g, \quad n \in \mathbb{N},
\end{gathered}
$$

because $\tilde{X}_{k}(\phi)(g)=\left.\frac{d}{d t} \phi\left(g \exp t X_{k}\right)\right|_{t=0}$. Hence, substituting $n$ by $2 m, m \geq 1$, and repeating the argument for every $1 \leq k \leq d$, one finds that for every chart $h: G \supseteq U \longrightarrow W \subseteq \mathbb{R}^{d}$, where $U$ and $W$ are open sets in their respective topologies and $W$ contains the origin of $\mathbb{R}^{d}$, the function $\psi: W \ni w \longmapsto f\left(V\left(h^{-1}(w)\right) x\right) \in \mathbb{R}$ is a continuous weak solution to the PDE

$$
\Delta_{m}(\psi)(w)=\sum_{k=1}^{d} f\left(V\left(h^{-1}(w)\right) d V\left(X_{k}\right)^{2 m} x\right), \quad w \in W
$$

where $\Delta_{m}:=\sum_{k=1}^{d} d V\left(X_{k}\right)^{2 m}$ is an elliptic operator. Since $x \in \mathcal{D}_{\infty}, m$ can be chosen arbitrarily large. Therefore, by [14, Theorem 1, page 190], $\psi$ is of class $\mathrm{C}^{\infty}$ on $W .{ }^{17} \mathrm{By}$ the arbitrariness of $h$, it follows that $g \longmapsto f(V(g) x)$ is of class $\mathrm{C}^{\infty}$ on $G$. But, then again, since $f$ is arbitrary, one may use [91, Lemma 1, page 47] to show that the map $g \longmapsto V(g) x$ is of class $\mathrm{C}^{\infty}$. This is because that theorem says, in particular, that smoothness with respect to the weak and the Mackey topology are equivalent. Therefore, since the polar of a set is always a convex, balanced and weakly compact (by the Banach-Alaoglu Theorem) subset of $\mathcal{X}^{\prime}$, the already proved fact that

$$
\sup _{f \in V_{p}^{\circ}}|f(y)|=p(y)
$$

\footnotetext{
${ }^{16}$ Remember that such field is defined by

$$
\tilde{X}_{k}(g):=D\left(L_{g}\right)(e)\left(X_{k}\right)
$$

for every $g \in G$, where $L_{g}: h \longmapsto g h$ is the operator of left-translation by $g$; see [56].

${ }^{17}$ This argumentation is possible because $W$ can be written as a union of bounded sets of $\mathbb{R}^{d}$ - note that the application of Theorem 1, mentioned above, requires a bounded domain of definition for the PDE.
} 
holds for all $p \in \Gamma$ and $y \in \mathcal{X}$ gives the desired smoothness of the function $g \longmapsto V(g) x .^{18}$ This shows the inclusion

$$
\mathcal{D}_{\infty} \subseteq C^{\infty}(V)
$$

proving that these sets are equal. Now it is easy to see that $C^{\infty}(V)$ is left invariant by all of the $d V(X)$ : write $X=\sum_{k=1}^{d} a_{k} X_{k}$, for some real numbers $a_{k}$. Then,

$$
d V(X)\left[C^{\infty}(V)\right] \subseteq \sum_{k=1}^{d} a_{k} d V\left(X_{k}\right)\left[C^{\infty}(V)\right]=\sum_{k=1}^{d} a_{k} d V\left(X_{k}\right)\left[\mathcal{D}_{\infty}\right] \subseteq \mathcal{D}_{\infty}=C^{\infty}(V)
$$

Hence, the following facts were proved (the second one was just mentioned) for a strongly continuous Lie group representation $g \longmapsto V(g)$ on a Hausdorff locally convex space:

1. the limit

$$
\lim _{t \rightarrow 0} \frac{V(\exp t X) x-x}{t}
$$

exists, for every $x \in C^{\infty}(V)$ and $X \in \mathfrak{g}$;

2. if $V: G \longrightarrow \mathcal{L}(\mathcal{X})$ is a strongly continuous locally equicontinuous representation of the Lie group $G$ and $\mathcal{X}$ is sequentially complete, then the generator $d V(X)$ is a closed, densely defined linear operator on $\mathcal{X}$;

3. if $\mathcal{X}$ is wafer complete, $C^{\infty}(V)$ is dense in $\mathcal{X}$;

4. if $\mathcal{X}$ is wafer complete, $C^{\infty}(V)$ is left invariant by the operators $d V(X)$, for all $X \in \mathfrak{g}$.

Therefore, when working with a strongly continuous Lie group representation $V$ on a Hausdorff locally convex space $\mathcal{X}$, some "good" basic general hypotheses which may be imposed a priori are that $\mathcal{X}$ is complete and that $V$ is locally equicontinuous.

\subsubsection{Lie Algebra Representations Induced by Group Representations}

Assume $\mathcal{X}$ is a complete Hausdorff locally convex space. Another important fact is that the application

$$
\partial V: \mathfrak{g} \ni X \longmapsto \partial V(X) \in \operatorname{End}\left(C^{\infty}(V)\right),
$$

where $\partial V(X):=\left.d V(X)\right|_{C^{\infty}(V)}$ and $\operatorname{End}\left(C^{\infty}(V)\right)$ denotes the algebra of all endomorphisms on $C^{\infty}(V)$ or, in other words, the linear operators defined on $C^{\infty}(V)$ with ranges contained in $C^{\infty}(V)$, is a representation of Lie algebras, so a strongly continuous representation of a Lie group always induces a representation of its Lie algebra (note that items 1 . and 4 ., above, show that this application is well-defined). This Lie algebra representation is called

\footnotetext{
${ }^{18}$ Note that, in order to apply such theorem, $\mathcal{X}$ must be a wafer complete locally convex space.
} 
the infinitesimal representation of $\boldsymbol{V}$. To see that it preserves commutators, fix $f \in \mathcal{X}^{\prime}$ and $x \in C^{\infty}(V)$. Then,

$$
\begin{gathered}
f([\partial V(X) \partial V(Y)-\partial V(Y) \partial V(X)] x) \\
=\left.\frac{d}{d t}\left[\left.\frac{d}{d s}[f(V(\exp t X) V(\exp s Y) x)]\right|_{s=0}\right]\right|_{t=0} \\
-\left.\frac{d}{d s}\left[\left.\frac{d}{d t}[f(V(\exp s Y) V(\exp t X) x)]\right|_{s=0}\right]\right|_{t=0} \\
=[\tilde{X} \tilde{Y}-\tilde{Y} \tilde{X}][f(V(\cdot) x)](e)=\widetilde{[X, Y]}[f(V(\cdot) x)](e) \\
=\left.\frac{d}{d t} f(V(\exp t[X, Y]) x)\right|_{t=0}=f(\partial V([X, Y]) x) .
\end{gathered}
$$

Since $f$ is arbitrary, a corollary of Hahn-Banach's Theorem gives

$$
[\partial V(X) \partial V(Y)-\partial V(Y) \partial V(X)](x)=\partial V([X, Y])(x),
$$

and arbitrariness of $x$ establishes the result. ${ }^{19}$ The linearity follows by an analogous argument.

Actually, defining

$$
\partial V\left(Y_{1} \ldots Y_{n}\right):=\partial V\left(Y_{1}\right) \ldots \partial V\left(Y_{n}\right)
$$

for $n \in \mathbb{N}$ and $Y_{i} \in \mathfrak{g}$, and extending this definition by linearity, one can define a unital homomorphism between the universal enveloping algebra of $\mathfrak{g}, \mathfrak{U}(\mathfrak{g})$ (which is, roughly speaking, the unital associative algebra formed by the real "noncommutative polynomials" in the elements of $\mathfrak{g})$, and a unital associative subalgebra of $\operatorname{End}\left(C^{\infty}(V)\right)$ that extends the original Lie algebra homomorphism $X \longmapsto \partial V(X)$ (the same notation was used to represent both the original morphism and its extension to $\mathfrak{U}(\mathfrak{g}))$. An analogous definition may be done with the operators $\partial V\left(Y_{k}\right)$ replaced by $d V\left(Y_{k}\right)$.

The results proved so far are motivators to define $C^{\infty}(V)$ as the domain of the representation $\partial V$, and will be denoted by Dom $\partial V$. Also, it is clear that, if $C^{\infty}(V)$ is substituted by a subspace $\mathcal{D}$ of $C^{\infty}(V)$ which is left invariant by all of the generators $d V(X), X \in \mathfrak{g}$, then it is also possible to define such infinitesimal representation by $\partial V(X):=\left.d V(X)\right|_{\mathcal{D}}$.

\subsubsection{Group Invariance and Cores}

The domain $C^{\infty}(V)$ also has some nice properties with respect to the group representation $V$. For example, $C^{\infty}(V)$ is also left invariant by all of the operators $V(g)$, because if $x \in C^{\infty}(V)$, then the application $G \ni w \longmapsto V(w) V(g) x \in \mathcal{X}$ is the composition of two

\footnotetext{
${ }^{19}$ This proof was an adaptation of the argument in [118, Proposition 10.1.6, page 263].
} 
functions of class $\mathrm{C}^{\infty}: w \longmapsto V(w) x$ and $w \longmapsto w g$.

Definition (Projective $\mathbf{C}^{\infty}$-Topology on Space of Smooth Vectors): Fix an ordered basis $\mathcal{B}:=\left(X_{k}\right)_{1 \leq k \leq d}$ for $\mathfrak{g}$, choose a fundamental system of seminorms $\Gamma$ for $\mathcal{X}$ and equip $C^{\infty}(V)$ with the topology defined by the family

$$
\left\{\rho_{p, n}: p \in \Gamma, n \in \mathbb{N}\right\}
$$

where

$$
\rho_{p, 0}(x):=p(x), \quad d V\left(X_{0}\right):=I
$$

and

$$
\rho_{p, n}(x):=\max \left\{p\left(d V\left(X_{i_{1}}\right) \ldots d V\left(X_{i_{n}}\right) x\right): 0 \leq i_{j} \leq d\right\}
$$

- this is called the projective $C^{\infty}$-topology on $C^{\infty}(V)$, and it does not depend upon neither the fixed basis $\mathcal{B}$ nor on the particular $\Gamma$. If $\mathcal{X}$ is sequentially complete and $V$ is locally equicontinuous, then each generator $d V\left(X_{k}\right)$ is closed, as mentioned before, and $C^{\infty}(V)$ becomes a complete Hausdorff locally convex space - to prove this, adapt the argument of [58, Corollary 1.1], using some of the facts already proved here and exploring the closedness of the operators $d V\left(X_{k}\right), 1 \leq k \leq d$.

Similarly, for each fixed $1 \leq k<\infty$, the subspace

$$
C^{k}(V):=\bigcap_{n=1}^{k} \bigcap\left\{\operatorname{Dom}\left[d V\left(X_{i_{1}}\right) \ldots d V\left(X_{i_{j}}\right) \ldots d V\left(X_{i_{n}}\right)\right]: X_{i_{j}} \in \mathcal{B}\right\}
$$

called the subspace of $\mathrm{C}^{k}$ vectors for the representation $V$, is a sequentially complete (respectively, complete) Hausdorff locally convex space when equipped with the $\mathrm{C}^{k}$-topology generated by the family

$$
\left\{\rho_{p, k}: p \in \Gamma\right\}
$$

of seminorms, if $\mathcal{X}$ is sequentially complete (respectively, complete) and $V$ is locally equicontinuous. Moreover, the operators

$$
V_{\infty}(g):=\left.V(g)\right|_{C^{\infty}(V)} \in \operatorname{End}\left(C^{\infty}(V)\right), \quad g \in G,
$$

are continuous with respect to this projective topology: suppose $\left\{x_{\alpha}\right\}_{\alpha \in \mathcal{A}}$ is a net in $C^{\infty}(V)$ converging to a certain $x \in C^{\infty}(V)$ with respect to the projective topology, and fix $X \in \mathfrak{g}$. Then,

$$
\begin{gathered}
d V(X) V\left(g^{-1}\right)\left(x_{\alpha}\right)=V\left(g^{-1}\right) V(g) d V(X) V\left(g^{-1}\right)\left(x_{\alpha}\right) \\
=V\left(g^{-1}\right) \lim _{t \rightarrow 0} \frac{V\left(g \exp t X g^{-1}\right)\left(x_{\alpha}\right)-x_{\alpha}}{t}=V\left(g^{-1}\right) \lim _{t \rightarrow 0} \frac{V(\exp [t \operatorname{Ad}(g)(X)])\left(x_{\alpha}\right)-x_{\alpha}}{t} \\
=V\left(g^{-1}\right) d V(\operatorname{Ad}(g)(X))\left(x_{\alpha}\right), \quad \alpha \in \mathcal{A},
\end{gathered}
$$


where in the third equality it was used the identity

$$
g \exp t X g^{-1}=\exp (\operatorname{Ad}(g)(t X)) \quad g \in G,
$$

to prove this - see [118, page 31]. Using the convergence hypothesis, the continuity of $V\left(g^{-1}\right)$ and taking limits on $\alpha$ yields

$$
\lim _{\alpha} d V(X) V\left(g^{-1}\right)\left(x_{\alpha}\right)=V\left(g^{-1}\right) d V(A d(g)(X))(x)
$$

proving the existence of a limit for $\left\{d V(X) V\left(g^{-1}\right)\left(x_{\alpha}\right)\right\}_{\alpha \in \mathcal{A}}$. Hence, closedness of $d V(X)$ proves the equality

$$
\lim _{\alpha} d V(X) V\left(g^{-1}\right)\left(x_{\alpha}\right)=d V(X) V\left(g^{-1}\right)(x)=V\left(g^{-1}\right) d V(A d(g)(X))(x) .
$$

Switching $X$ by a basis element $X_{k}$ and iterating this process proves that for a monomial $d V\left(X_{i_{1}}\right) \ldots d V\left(X_{i_{n}}\right)$ on the operators $\left\{d V\left(X_{k}\right)\right\}_{1 \leq k \leq d}$ of arbitrary size $n$, the convergence of

$$
\lim _{\alpha} d V\left(X_{i_{1}}\right) \ldots d V\left(X_{i_{n}}\right) V\left(g^{-1}\right)\left(x_{\alpha}\right)=d V\left(X_{i_{1}}\right) \ldots d V\left(X_{i_{n}}\right) V\left(g^{-1}\right)(x)
$$

is guaranteed, establishing the desired result.

Definition (Cores): If $T:$ Dom $T \subseteq \mathcal{X} \longrightarrow \mathcal{X}$ is a closed linear operator and $\mathcal{D} \subseteq$ Dom $T$ is a linear subspace of Dom $T$ such that

$$
\overline{\left.T\right|_{\mathcal{D}}}=T
$$

then $\mathcal{D}$ is called a core for $T$.

Before proceeding, a few words about the use of some notations must be said. Consider a Lie algebra $\mathfrak{g}$ with an ordered basis $\left(B_{k}\right)_{1 \leq k \leq d}$. Throughout the whole manuscript, whenever a monomial $B^{\mathrm{u}}$ of size $n \geq 1$ in the elements of $\left(B_{k}\right)_{1 \leq k \leq d}$ is considered, it is understood that $\mathrm{u}=\left(\mathrm{u}_{j}\right)_{j \in\{1, \ldots, n\}}$ is a function from $\{1, \ldots, n\}$ to $\{1, \ldots, d\}$ and $B^{\mathrm{u}}:=$ $B_{\mathrm{u}_{1}} \ldots B_{\mathrm{u}_{n}}$ is an element of the complexified universal enveloping algebra $(\mathfrak{U}[\mathfrak{g}])_{\mathbb{C}}:=\mathfrak{U}[\mathfrak{g}]+$ $i \mathfrak{U}[\mathfrak{g}]$. Therefore, $B^{\mathrm{u}}$ can be thought of as an unordered monomial, or a noncommutative monomial. ${ }^{20}$ The size of the monomial is defined to be $|\mathbf{u}|=n$ so, roughly speaking, it is the size of the "word" $B_{\mathrm{u}_{1}} \ldots B_{\mathrm{u}_{n}}$. For a matter of convenience, the size of a linear combination of monomials is defined to be the size of the "biggest" monomial composing the sum. However, the following question arises: could this sum be written in another way, so that the associated size is different? It may very well be the case that the basis elements share an extra relation, thus causing this kind of ambiguity: for example, if $X$ and $Y$ are

\footnotetext{
${ }^{20}$ The expression "unordered" refers to the fact that the sequence $\left(\mathbf{u}_{1}, \ldots, \mathbf{u}_{n}\right)$ of numbers is not (necessarily) non-decreasing.
} 
elements of a Lie algebra with basis $\{X, Y,[X, Y]\}$, then $[X, Y]=X Y-Y X$ has size 2 , but also size 1, depending on how it is written. Therefore, the concept of size is not an intrinsic one, and is intimately related to how the sum was written. The same cannot be said about the definition of order of an element in $\mathfrak{U}[\mathfrak{g}]$ : by the Poincaré-Birkhoff-Witt Theorem [77, Theorem 3.8, page 217] together with an inductive procedure on the argument sketched at the beginning of Section 1.5, one sees that every linear combination of monomials $P$ can be written in a unique way as a polynomial on the variables $\left(B_{k}\right)_{1 \leq k \leq d}$ of the form

$$
\sum_{|\alpha| \leq m} c_{\alpha} B_{1}^{\alpha_{1}} \ldots B_{k}^{\alpha_{k}} \ldots B_{d}^{\alpha_{d}}
$$

where $c_{\alpha^{\prime}} \neq 0$ for some multi-index $\alpha^{\prime}$ of order $m$. In this case, the order of $P$ is defined to be $\left|\alpha^{\prime}\right|=m$, the order of the multi-index $\alpha^{\prime}$, and it does not depend on the particular choice of the basis. Note that the notations were carefully chosen, in order to avoid confusions. The symbols $\mathrm{u}, \mathrm{v}$ and $\mathrm{w}$ will always be employed to indicate the use of an unordered monomial, while the greek letters $\alpha, \beta$ and $\gamma$ will denote multi-indices, following the usual convention for ordered monomials. The use of the noncommutative monomial notation will occur in Section 1.5, Theorem 2.5 and in the proof of some of the exponentiation theorems. As an abbreviation, they will usually be referred to as "monomials", rather than "noncommutative monomials".

To prove a last useful result concerning the representation $\partial V$ the following two theorems, which go in the spirit of [102, Corollary 1.2] and [102, Corollary 1.3], will be proved.

Theorem 1.2.4.1: Let $g \longmapsto V(g)$ be a strongly continuous locally equicontinuous representation of a Lie group $G$, with Lie algebra $\mathfrak{g}$, on a complete Hausdorff locally convex space $(\mathcal{X}, \Gamma)$, whose topology will be denoted by $\tau$. Suppose that $\mathcal{D}$ is a dense subspace of $\mathcal{X}$ which is a closed subspace of $C^{\infty}(V)$ when equipped with the projective $C^{\infty}$ topology $\tau_{\infty}$. Then, if

$$
V(g)[\mathcal{D}] \subseteq \mathcal{D}, \quad g \in G,
$$

that is, if $\mathcal{D}$ is group invariant, $\mathcal{D}$ must be a core for every closed operator

$$
\overline{\left.d V\left(X_{i_{1}}\right) \ldots d V\left(X_{i_{n}}\right)\right|_{C^{n}(V)}}
$$

in the basis elements $\left\{d V\left(X_{k}\right)\right\}_{1 \leq k \leq d}$, for all $n \in \mathbb{N}, n \geq 1$. More generally, if $L \in \mathfrak{U}(\mathfrak{g})$ is an element of the universal enveloping algebra of size at most $n$, then $\mathcal{D}$ is a core for $\overline{\left.d V(L)\right|_{C^{n}(V)}}$, and if $L \in \mathfrak{U}(\mathfrak{g})$ is any element, then $\mathcal{D}$ is a core for $\overline{\left.d V(L)\right|_{C^{\infty}(V)}}$.

Proof of Theorem 1.2.4.1: To see $g \longmapsto \phi(g) V(g) x$ is a compactly supported weaklycontinuous function from $\boldsymbol{G}$ to $\mathcal{D}$, for all $x \in \mathcal{D}$ and $\phi \in C_{c}^{\infty}(G)$, it will be proved that $g \longmapsto V(g) x$ is a smooth function from $G$ to $\left(\mathcal{D}, \tau_{\infty}\right)$. Following the idea of $[102$, 
Proposition 1.2], let $X_{i_{1}} \ldots X_{i_{n}}$ be a monomial in the basis elements. Then, it is sufficient to show $g \longmapsto \partial V\left(X_{i_{1}} \ldots X_{i_{n}}\right) V(g) x$ is a smooth function from $G$ to $(\mathcal{X}, \tau)$. Consider the analytic coordinate system $h(t) \longmapsto t$ around the identity $e$ of $G$ defined by

$$
h(t):=\exp \left(t_{1} X_{1}\right) \ldots \exp \left(t_{d} X_{d}\right), \quad t:=\left(t_{k}\right)_{1 \leq k \leq d} \in \mathbb{R}^{d} .
$$

The function $(s, t) \longmapsto V(h(s) h(t)) x$ is $\mathrm{C}^{\infty}$ from $\mathbb{R}^{2 d}$ to $\mathcal{X}$, because $C^{\infty}(V)$ is left invariant by $V$ and by the generators $d V\left(X_{k}\right), 1 \leq k \leq d$. Therefore, since

$$
\partial V\left(X_{i_{1}} \ldots X_{i_{n}}\right) V(h(t)) x=\left.\frac{\partial}{d s_{i_{1}}} \ldots \frac{\partial}{d s_{i_{n}}} V(h(s) h(t)) x\right|_{s=0}, \quad t \in \mathbb{R}^{d},
$$

the result follows.

Hence, in particular, if $\left\{g_{m}\right\}_{m \in \mathbb{N}}$ is a sequence converging to $g_{0} \in G$, the sequence

$$
\left\{d V\left(X_{i_{1}}\right) \ldots d V\left(X_{i_{n}}\right) V\left(g_{m}\right) x\right\}_{m \in \mathbb{N}}
$$

converges to $d V\left(X_{i_{1}}\right) \ldots d V\left(X_{i_{n}}\right) V\left(g_{0}\right) x$, and so

$$
f^{\prime}\left(V\left(g_{\alpha}\right) x\right) \longrightarrow f^{\prime}\left(V\left(g_{0}\right) x\right)
$$

for every $f^{\prime} \in \mathcal{D}^{\prime}$, by the definition of the projective $\mathrm{C}^{\infty}$-topology. This means $g \longmapsto V(g) x$ is a weakly continuous function from $G$ to $\mathcal{D}$. Therefore, the proof made in the paragraphs where the Gärding domain was defined guarantees the existence of the integral $\int_{G} \phi(g) V(g) x d g$ as an element of $\mathcal{D}$ (note that the hypotheses of group invariance and of wafer completeness - actually, completeness - of $\mathcal{D}$ with respect to the projective topology were essential).

For the next step, it is necessary to see that if $x \in \mathcal{X}$ and $\phi \in C_{c}^{\infty}(G)$, then $x(\phi) \in \mathcal{D}$. In fact, fixing $p \in \Gamma$ and using what was proved in 1.2.1,

$$
p(y(\phi)) \leq \operatorname{vol}(\operatorname{supp} \phi)\left[\sup _{g \in \operatorname{supp} \phi}|\phi(g)|\right]\left[\sup _{g \in \operatorname{supp} \phi} p(V(g) y)\right], \quad y \in \mathcal{X} .
$$

Choose a net $\left\{x_{\alpha}\right\}_{\alpha \in \mathcal{A}}$ in $\mathcal{D}$ converging to $x$ in $\mathcal{X}$, whose existence is guaranteed by the denseness hypothesis. Then, it will be proved that

$$
x_{\alpha}(\phi) \longrightarrow x(\phi), \quad \phi \in C_{c}^{\infty}(G)
$$

with respect to $\tau_{\infty}$ : by hypothesis, $V$ is locally equicontinuous, so making $K:=\operatorname{supp} \phi$, there exists $M_{p, K}>0$ and $q \in \Gamma$ satisfying

$$
p(V(g) x) \leq M_{p, K} q(x), \quad g \in K, x \in \mathcal{X} .
$$


Therefore,

$$
p\left(x_{\alpha}(\phi)-x(\phi)\right)=p\left(\left(x_{\alpha}-x\right)(\phi)\right) \leq \operatorname{vol}(K)\left[\sup _{g \in K}|\phi(g)|\right] M_{p, K} q\left(x_{\alpha}-x\right) \longrightarrow 0,
$$

for all $\phi \in C_{c}^{\infty}(G)$. Repeating the argument developed in the paragraph about the Gårding domain, the following equality is obtained:

$$
\begin{gathered}
d V(X)(y(\phi))=\lim _{t \rightarrow 0} \frac{V(\exp t X)(y(\phi))-y(\phi)}{t} \\
=\int_{G} \lim _{t \rightarrow 0} \frac{\phi([\exp -t X] g)-\phi(g)}{t} V(g) y d g=-y(\tilde{X}(\phi)),
\end{gathered}
$$

for all $y \in \mathcal{X}, \phi \in C_{c}^{\infty}(G)$ and $X \in \mathfrak{g}$. An iteration of this argument gives

$$
d V\left(X_{i_{1}}\right) \ldots d V\left(X_{i_{n}}\right)(y(\phi))=(-1)^{n} y\left(\phi_{n}\right), \quad n \in \mathbb{N}, y \in \mathcal{X},
$$

where $X_{i_{k}} \in \mathfrak{g}, 1 \leq k \leq n$, and

$$
\phi_{n}(g):=\tilde{X}_{i_{1}} \ldots \tilde{X}_{i_{n}}(\phi)(g)
$$

is a function in $C_{c}^{\infty}(G)$. Hence, if $\phi \in C_{c}^{\infty}(G)$ is fixed,

$$
\begin{gathered}
p\left(d V\left(X_{i_{1}}\right) \ldots d V\left(X_{i_{n}}\right)\left(x_{\alpha}(\phi)-x(\phi)\right)\right)=p\left(d V\left(X_{i_{1}}\right) \ldots d V\left(X_{i_{n}}\right)\left(\left(x_{\alpha}-x\right)(\phi)\right)\right) \\
=p\left(\left(x_{\alpha}-x\right)\left(\phi_{n}\right)\right) \longrightarrow 0
\end{gathered}
$$

for all $p \in \Gamma$ and $n \in \mathbb{N}$. Therefore, it follows that the net $\left\{x_{\alpha}(\phi)\right\}_{\alpha \in \mathcal{A}}$ in $\mathcal{D}$ actually converges to $x(\phi)$ with respect to the (stronger) projective $\mathrm{C}^{\infty}$ topology. Since $\mathcal{D}$ is complete with respect to this topology, $x(\phi) \in \mathcal{D}$, proving the desired assertion.

Now, the main statement of the theorem can be proved: fix $n \in \mathbb{N}$,

$$
x \in C^{n}(V) \subseteq \operatorname{Dom} d V\left(X_{i_{1}}\right) \ldots d V\left(X_{i_{n}}\right)
$$

and a left invariant Haar measure on $G$. Also, fix a sequence $\left\{\phi_{m}\right\}_{m \in \mathbb{N}}$ of nonnegative functions in $C_{c}^{\infty}(G)$ with

$$
\int_{G} \phi_{m} d g=1, \quad m \in \mathbb{N}
$$

and with the property that, for every open neighborhood $U$ of the origin, there exists $m_{U} \in \mathbb{N}$ such that supp $\phi_{m} \subseteq U$, for all $m \geq m_{U}$. Then, $x\left(\phi_{m}\right)$ converges to $x$ with respect to $\tau$. The fact that the operators $d V(X), X \in \mathfrak{g}$, are continuous with respect to the $\mathrm{C}^{\infty}$-topology ensures that

$$
d V\left(X_{i_{1}}\right) \ldots d V\left(X_{i_{n}}\right)\left(x\left(\phi_{m}\right)-x\right)=\int_{G} \phi_{m}(g) d V\left(X_{i_{1}}\right) \ldots d V\left(X_{i_{n}}\right)[V(g) x-x] d g,
$$


for all $m \in \mathbb{N}$. Also,

$$
g \longmapsto d V\left(X_{j_{1}}\right) \ldots d V\left(X_{j_{n}}\right) V(g) x
$$

is a continuous function from $G$ to $\mathcal{X}$, where $X_{j_{k}}$ is a basis element, so

$$
\begin{gathered}
p\left(d V\left(X_{i_{1}}\right) \ldots d V\left(X_{i_{n}}\right)\left(x\left(\phi_{m}\right)-x\right)\right) \\
\leq\left[\int_{G} \phi_{m}(g) d g\right] \sup _{g \in \operatorname{supp} \phi_{m}} p\left(d V\left(X_{i_{1}}\right) \ldots d V\left(X_{i_{n}}\right)[V(g) x-x]\right), \quad p \in \Gamma,
\end{gathered}
$$

and $p\left(d V\left(X_{i_{1}}\right) \ldots d V\left(X_{i_{n}}\right)\left(x\left(\phi_{m}\right)-x\right)\right) \longrightarrow 0$.

Because $x\left(\phi_{m}\right)$ belongs to $\mathcal{D}$, for all $m \in \mathbb{N}$, this proves that

$$
\left.d V\left(X_{i_{1}}\right) \ldots d V\left(X_{i_{n}}\right)\right|_{C^{n}(V)} \subset \overline{\left.d V\left(X_{i_{1}}\right) \ldots d V\left(X_{i_{n}}\right)\right|_{\mathcal{D}}},
$$

so

$$
\overline{\left.d V\left(X_{i_{1}}\right) \ldots d V\left(X_{i_{n}}\right)\right|_{C^{n}(V)}} \subset \overline{\left.d V\left(X_{i_{1}}\right) \ldots d V\left(X_{i_{n}}\right)\right|_{\mathcal{D}}} .
$$

Since the other inclusion is immediate, the result is proved.

The proofs for a general $L \in \mathfrak{U}(\mathfrak{g})$ and for $C^{\infty}(V)$ are analogous, and need simple adaptations in the last two paragraphs.

Still under the hypotheses of Theorem 1.2.4.1, if $\mathcal{D}$ is not assumed to be complete with respect to $\tau_{\infty}$, then its closure with respect to $\tau_{\infty}$ will also be left invariant by the operators $\left.V(g)\right|_{C_{\infty}(V)}$, because they are all continuous with respect to $\tau_{\infty}$, as was already noted at the beginning of this subsection. Hence, the hypothesis of completeness on $\mathcal{D}$ may be dropped, without affecting the resulting conclusions.

Corollary 1.2.4.2: Under the same hypotheses of Theorem 1.2.4.1, $\mathcal{D}$ is a core for the operators $\overline{d V\left(X_{k}\right)^{n}}$, for all $n \in \mathbb{N}$ and $1 \leq k \leq d$. In particular, it is a core for $d V\left(X_{k}\right)$, for all $1 \leq k \leq d$.

Proof of Corollary 1.2.4.2: Fix $1 \leq k \leq d$. The proof is a repetition of the previous one, except that in the last paragraph one has to substitute $C^{n}(V)$ by Dom $\overline{d V\left(X_{k}\right)^{n}}$, which is a complete Hausdorff locally convex space when equipped with the topology generated by the family $\left\{\rho_{p}^{(n)}: p \in \Gamma\right\}$ of seminorms, where

$$
\rho_{p}^{(n)}(x):=\max \left\{p(x), p\left(\overline{d V\left(X_{k}\right)^{n}}(x)\right)\right\}, \quad x \in \mathcal{X} .
$$

Then, in this context, the operator $\overline{d V\left(X_{k}\right)^{n}}$ will be continuous, and the rest of the proof will follow analogously. 
The next lemma is an adaptation of [30, Corollary 3.1.7, page 167] to $\Gamma$-semigroups of bounded type on locally convex spaces, and will be used in the proof of Theorem 2.15. It is a weaker version of Lemma 1.2.4.1, because it restricts to $\Gamma$-semigroups of bounded type, but it is still very useful and its proof is much simpler than the one given in Lemma 1.2.4.1:

Lemma 1.2.4.3: Let $(\mathcal{X}, \Gamma)$ be a complete Hausdorff locally convex space and $t \longmapsto S(t)$ a $\Gamma$-semigroup of bounded type $w$, whose generator is $T$. Suppose $\mathcal{D} \subseteq D$ om $T$ is a dense subspace of $\mathcal{X}$ which is left invariant by $S$ - in other words, $S(t)[\mathcal{D}] \subseteq \mathcal{D}$, for all $t \geq 0$. Then, $\mathcal{D}$ is a core for $T$.

Proof of Lemma 1.2.4.3: Define $\tilde{T}:=\overline{\left.T\right|_{\mathcal{D}}}$, so that $\tilde{T} \subset T$ (this holds because $S$ is locally equicontinuous, so $T$ is closed - see [78, Proposition 1.4]), and fix a real $\lambda$ satisfying $\lambda>w$. Then, [8, Theorem 3.3, page 172] shows that formula

$$
\mathrm{R}(\lambda, T) x=\int_{0}^{+\infty} e^{-\lambda t} S(t) x d t
$$

holds for all $x \in \mathcal{X}$, where the integral is strongly convergent with respect to the topology induced by $\Gamma$. Fix $x \in \mathcal{D}$. There exist Riemann sums of the form

$$
\sum_{k=1}^{N} e^{-\lambda t_{k}} S\left(t_{k}\right) x\left(t_{k+1}-t_{k}\right)
$$

for the integral above (which are all elements of $\mathcal{D}$, by the invariance hypothesis) which converge to $\mathrm{R}(\lambda, T) x$ and possess the property that the Riemann sums

$$
\sum_{k=1}^{N} e^{-\lambda t_{k}} S\left(t_{k}\right)[(\lambda I-T) x]\left(t_{k+1}-t_{k}\right)
$$

converge to $\mathrm{R}(\lambda, T)[(\lambda I-T) x]=x$. Closedness of $\tilde{T}$ imply $\mathrm{R}(\lambda, T) x \in \operatorname{Dom} \tilde{T}$ and $(\lambda I-\tilde{T}) \mathrm{R}(\lambda, T) x=x$, so $\mathcal{D} \subseteq \operatorname{Ran}(\lambda I-\tilde{T})$. Therefore, $\operatorname{Ran}(\lambda I-\tilde{T})$ is dense in $\mathcal{X}$. Since $\mathrm{R}(\lambda, \tilde{T})$ is continuous and $\tilde{T}$ is closed, $\operatorname{Ran}(\lambda I-\tilde{T})$ is also closed, so $\operatorname{Ran}(\lambda I-\tilde{T})=\mathcal{X}$.

Now, let $x \in \operatorname{Dom} T$. By what was just proved, there exists $y \in \operatorname{Dom} \tilde{T}$ such that

$$
(\lambda I-\tilde{T}) x=(\lambda I-T) x=(\lambda I-\tilde{T}) y
$$

which implies $(\lambda I-\tilde{T})(x-y)=0$. But the operator $\lambda I-\tilde{T}$, being a restriction of an injective operator, is itself injective, showing that $x=y \in \operatorname{Dom} \tilde{T}$. This proves $\tilde{T}=T$ and ends the proof. 


\subsection{Dissipative and Conservative Operators}

Definitions (Dissipative and Conservative Operators) [3, Definition 3.9]: Let $\mathcal{X}$ be a Hausdorff locally convex space and $\Gamma$ a fundamental system of seminorms for $\mathcal{X}$. A linear operator $T: D o m T \subseteq \mathcal{X} \longrightarrow \mathcal{X}$ is called $\boldsymbol{\Gamma}$-dissipative if, for every $p \in \Gamma, \mu>0$ and $x \in \operatorname{Dom} T$,

$$
p((\mu I-T) x) \geq \mu p(x) .
$$

Since $\mathcal{X}$ is Hausdorff, this implies $\mu I-T$ is an injective linear operator, for every $\mu>0$. $T$ is called $\boldsymbol{\Gamma}$-conservative if both $T$ and $-T$ are $\Gamma$-dissipative or, equivalently, if the inequality

$$
p((\mu I-T) x) \geq|\mu| p(x)
$$

holds for all $p \in \Gamma, \mu \in \mathbb{R}$ and $x \in$ Dom $T$. Note that every $\Gamma$-conservative operator is $\Gamma$-dissipative and that the definitions of dissipativity and conservativity both depend on the particular choice of the fundamental system of seminorms - see Remark 3.10 of [3], for an illustration of this fact.

The next lemma is a straightforward corollary of $[126 \text {, Corollary } 1 \text {, page } 241]^{21}$ which will be important to provide a better understanding of the spectrum of the generator of an equicontinuous one-parameter group:

Lemma 1.3.1: Let $\mathcal{X}$ be a sequentially complete Hausdorff locally convex space. If $A$ is the infinitesimal generator of an equicontinuous group, then

$$
\mathbb{C} \backslash i \mathbb{R}=\mathbb{C} \backslash\{\lambda \in \mathbb{C}: \text { Re } \lambda=0\} \subseteq \rho(A) .
$$

Proof of Lemma 1.3.1: It is clear that the semigroups $V_{+}$and $V_{-}$defined by $V_{+}(t):=V(t)$ and $V_{-}(t):=V(-t)$, for all $t \geq 0$, are equicontinuous and have $A$ and $-A$ as their generators, respectively. Then, [126, Corollary 1, page 241] applied to $A$ gives the information

$$
\{\lambda \in \mathbb{C}: \operatorname{Re} \lambda>0\} \subseteq \rho(A) .
$$

On the other hand, applying them to $-A$ gives

$$
\{\lambda \in \mathbb{C}: \operatorname{Re} \lambda>0\} \subseteq \rho(-A)
$$

${ }^{21}[\mathbf{1 2 6}$, Corollary 1, page 241]: Let $\mathcal{Y}$ be a sequentially complete Hausdorff locally convex space and $B$ the generator of an equicontinuous semigroup $t \longmapsto T_{t}$ on $\mathcal{Y}$. Then, the right-half plane of $\mathbb{C}$ is in the resolvent set of $B$ and one has

$$
\mathrm{R}(\lambda, B) y=\int_{0}^{+\infty} \exp (-\lambda t) T_{t} y d t, \quad \operatorname{Re} \lambda>0, y \in \mathbb{Y}
$$


or, in other words,

$$
\{\lambda \in \mathbb{C}: \operatorname{Re} \lambda<0\} \subseteq \rho(A) .
$$

This proves the desired result.

Definition ( $\Gamma$-Contractively Equicontinuous Semigroups and $\Gamma$-Isometrically Equicontinuous Groups): Suppose $\mathcal{X}$ is sequentially complete, $t \longmapsto V(t)$ is an equicontinuous one-parameter semigroup on $\mathcal{X}$ (as in the definition given in Section 1.1) with generator $T$ and define, for each $p \in \Gamma$, the seminorm

$$
\tilde{p}(x):=\sup _{t \geq 0} p(V(t) x), \quad x \in \mathcal{X} .
$$

Then, their very definitions show that

$$
p(x) \leq \tilde{p}(x) \leq M_{p} q(x) \leq M_{p} \tilde{q}(x), \quad x \in \mathcal{X},
$$

proving that the families $\Gamma$ and $\tilde{\Gamma}:=\{\tilde{p}: p \in \Gamma\}$ are equivalent, in the sense that they generate the same topology of $\mathcal{X}$ (see also [2, Remark 2.2(i)]). It follows also that

$$
\tilde{p}(V(t) x) \leq \tilde{p}(x), \quad \tilde{p} \in \tilde{\Gamma}, x \in \mathcal{X}
$$

which means $t \longmapsto V(t)$ is a $\tilde{\boldsymbol{\Gamma}}$-contractively equicontinuous semigroup, according to the terminology introduced at the bottom of page 935 of [3]. By the fact that $\{z \in \mathbb{C}:$ Re $z>0\} \subseteq \rho(T)$ and the formula

$$
R(\lambda, T) x=\int_{0}^{+\infty} \exp (-\lambda t) V(t) x d t, \quad \text { Re } \lambda>0, x \in \mathcal{X}
$$

(see [3, Remark 3.12]), it follows that

$$
\tilde{p}(R(\lambda, T) x) \leq \frac{1}{\operatorname{Re} \lambda} \tilde{p}(x), \quad \tilde{p} \in \tilde{\Gamma}, \operatorname{Re} \lambda>0, x \in \mathcal{X} .
$$

In particular,

$$
\tilde{p}(R(\lambda, T) x) \leq \frac{1}{\lambda} \tilde{p}(x), \quad \tilde{p} \in \tilde{\Gamma}, \lambda>0, x \in \mathcal{X},
$$

meaning $T$ is a $\tilde{\Gamma}$-dissipative operator.

If $t \longmapsto V(t)$ is an equicontinuous one-parameter group these conclusions also follow in perfect analogy by making

$$
\tilde{p}(x):=\sup _{t \in \mathbb{R}} p(V(t) x), \quad x \in \mathcal{X}
$$

for all $p \in \Gamma$. In this case,

$$
\tilde{p}(V(t) x)=\tilde{p}(x), \quad \tilde{p} \in \tilde{\Gamma}, x \in \mathcal{X}
$$


so $V$ will be called a $\tilde{\boldsymbol{\Gamma}}$-isometrically equicontinuous group. Moreover, the semigroups $V_{+}$and $V_{-}$defined by $V_{+}(t):=V(t)$ and $V_{-}(t):=V(-t)$, for all $t \geq 0$, are equicontinuous. Applying the above results to $V_{+}$and $V_{-}$gives

$$
R(\lambda, \pm T) x=\int_{0}^{+\infty} \exp (-\lambda t) V_{ \pm}(t) x d t, \quad x \in \mathcal{X}
$$

so

$$
\tilde{p}(R(\lambda, \pm T) x) \leq \frac{1}{\operatorname{Re} \lambda} \tilde{p}(x), \quad \tilde{p} \in \tilde{\Gamma}, \text { Re } \lambda>0, x \in \mathcal{X}
$$

On the other hand, if Re $\lambda<0$, applying the relation $R(\lambda, T)=-R(-\lambda,-T)$ yields

$$
\tilde{p}(R(\lambda, T) x)=\tilde{p}(R(-\lambda,-T) x) \leq \frac{1}{-\operatorname{Re} \lambda} \tilde{p}(x), \quad p \in \Gamma, x \in \mathcal{X} .
$$

Hence,

$$
\tilde{p}(R(\lambda, T) x) \leq \frac{1}{|\operatorname{Re} \lambda|} \tilde{p}(x), \quad p \in \Gamma, \operatorname{Re} \lambda \neq 0, x \in \mathcal{X}
$$

In particular,

$$
\tilde{p}(R(\lambda, T) x) \leq \frac{1}{|\lambda|} \tilde{p}(x), \quad p \in \Gamma, \lambda \in \mathbb{R} \backslash\{0\}, x \in \mathcal{X}
$$

proving that $T$ is $\tilde{\Gamma}$-conservative, where $\tilde{\Gamma}:=\{\tilde{p}: p \in \Gamma\}$.

These particular choices of fundamental systems of seminorms will be very useful for the future proofs.

The next lemma slightly strengthens [8, Theorem 4.2, page 173] (see Section 1.4, below):

Lemma 1.3.2: Let $(\mathcal{X}, \Gamma)$ be a Hausdorff locally convex space. If $T$ is a densely defined linear operator with the property that, for every $p \in \Gamma$, there exist $\sigma_{p} \geq 0$ and $M_{p}>0$ such that, for all $\mu>\sigma_{p}$ and $x \in$ Dom $T$,

$$
p((\mu I-T) x) \geq M_{p}^{-1}\left(\mu-\sigma_{p}\right) p(x),
$$

then $T$ is closable.

Proof of Lemma 1.3.2: Let $\left\{x_{\alpha}\right\}$ and $\left\{T\left(x_{\alpha}\right)\right\}$ be nets in $\mathcal{X}$ such that

$$
x_{\alpha} \longrightarrow 0
$$

and

$$
T\left(x_{\alpha}\right) \longrightarrow y
$$


in $\mathcal{X}$. Fix $p \in \Gamma$. By the hypotheses, one has for all $\mu>\sigma_{p}$ and $x^{\prime} \in \operatorname{Dom} T$ that

$$
p\left(\left(I-\frac{1}{\mu} T\right)\left(x_{\alpha}+\frac{1}{\mu} x^{\prime}\right)\right) \geq M_{p} \frac{\mu-\sigma_{p}}{\mu} p\left(x_{\alpha}+\frac{1}{\mu} x^{\prime}\right) .
$$

Hence,

$$
p\left(x_{\alpha}+\frac{1}{\mu} x^{\prime}-\frac{1}{\mu} T\left(x_{\alpha}\right)-\frac{1}{\mu^{2}} T\left(x^{\prime}\right)\right) \geq M_{p} \frac{\mu-\sigma_{p}}{\mu} p\left(x_{\alpha}+\frac{1}{\mu} x^{\prime}\right) .
$$

By taking limits (in $\mathcal{X}$ ) on $\alpha$ and multiplying by $\mu$, one obtains

$$
p\left(x^{\prime}-y-\frac{1}{\mu} T\left(x^{\prime}\right)\right) \geq M_{p} \frac{\mu-\sigma_{p}}{\mu} p\left(x^{\prime}\right) .
$$

Sending $\mu$ to $+\infty$ and using the density of $\operatorname{Dom} T$ in $\mathcal{X}$, it follows that $p(y)=0$. Since $p \in \Gamma$ is arbitrary, $\Gamma$ is a fundamental system of seminorms and $\mathcal{X}$ is Hausdorff, $y=0$. Hence, $T$ is closable.

Corollary 1.3.3: If $\mathcal{X}$ is a Hausdorff locally convex space with a fundamental system of seminorms $\Gamma$ and $T$ is a $\Gamma$-dissipative operator on $\mathcal{X}$, then $T$ is closable.

Proof of Corollary 1.3.3: The result follows at once by Lemma 1.3, putting $M_{p}=1$ and $\sigma_{p}=0$, for all $p \in \Gamma$.

\subsection{The Kernel Invariance Property (KIP), Projective Analytic Vectors}

Now, a concept which will appear very frequently throughout this manuscript is going to be introduced.

Definition (The Kernel Invariance Property (KIP)): If $(\mathcal{X}, \Gamma)$ is a Hausdorff locally convex space, define for each $p \in \Gamma$ the closed subspace

$$
N_{p}:=\{x \in \mathcal{X}: p(x)=0\},
$$

often referred to as the kernel of the seminorm $p$, and the quotient map $\pi_{p}: \mathcal{X} \ni x \longmapsto$ $[x]_{p} \in \mathcal{X} / N_{p}$. Then, $\mathcal{X} / N_{p}$ is a normed space with respect to the norm $\left\|[x]_{p}\right\|_{p}:=p(x)$, and is not necessarily complete. Denote its completion by $\mathcal{X}_{p}:=\overline{\mathcal{X} / N_{p}}$. A densely defined linear operator $T:$ Dom $T \subseteq \mathcal{X} \longrightarrow \mathcal{X}$ is said to possess the kernel invariance property (KIP) with respect to $\Gamma$ if it leaves their seminorms' kernels invariant, that is,

$$
T\left[\operatorname{Dom} T \cap N_{p}\right] \subseteq N_{p}, \quad p \in \Gamma .^{22}
$$

\footnotetext{
${ }^{22}[8]$ calls them "compartmentalized operators".
} 
1.4 The Kernel Invariance Property (KIP), Projective Analytic Vectors

If this property is fulfilled, then the linear operators

$$
T_{p}: \pi_{p}[\operatorname{Dom} T] \subseteq \mathcal{X}_{p} \longrightarrow \mathcal{X}_{p}, \quad T_{p}:[x]_{p} \longmapsto[T(x)]_{p}, \quad p \in \Gamma, x \in \operatorname{Dom} T
$$

on the quotients are well-defined, and their domains are dense in each $\mathcal{X}_{p}$. If a oneparameter semigroup $t \longmapsto V(t)$ is such that all of the operators $V(t)$ possess the (KIP) with respect to $\Gamma$, it will be said that the semigroup $V$ possesses the (KIP) with respect to $\Gamma$. The terminology is analogous for the representation $g \longmapsto V(g)$ of a Lie group.

Note that, if $\Gamma$ is a fundamental system of seminorms with respect to which $t \longmapsto V(t)$ is $\Gamma$-contractively equicontinuous, then $t \longmapsto V(t)$ leaves all the $N_{p}$ 's invariant. Hence, $T$ possesses the kernel invariance property with respect to $\Gamma$, by the definition of infinitesimal generator and by closedness of the $N_{p}$ 's. As a corollary of what was proved in Section 1.3 and this last observation, it follows that for any generator $T$ of a one-parameter equicontinuous semigroup $t \longmapsto V(t)$ acting on a sequentially complete Hausdorff locally convex space $\mathcal{X}$ there exists a fundamental system of seminorms $\tilde{\Gamma}$ for $\mathcal{X}$ such that $T$ is $\tilde{\Gamma}$-dissipative, $T$ has the (KIP) with respect to $\tilde{\Gamma}$ and $V$ is a $\tilde{\Gamma}$-contractively equicontinuous semigroup - analogously, this also holds for one-parameter equicontinuous groups. Also, an obvious fact is that if a linear operator $T$ possesses an extension having the (KIP) with respect to some fundamental system of seminorms, then $T$ also possesses this property (in particular, infinitesimal pregenerators of equicontinuous semigroups always possess the (KIP) with respect to a certain fundamental system of seminorms). In a locally $\mathrm{C}^{*}$-algebra (they will be properly defined later, in Chapter 3), for example, it is proved inside [13, Proposition $2]$ that an everywhere defined $*$-derivation satisfies the kernel invariance property with respect to any saturated fundamental system of seminorms. Hence, a $*$-derivation $\delta$ which is the pointwise limit of a net of globally defined $*$-derivations - that is, if there exists a net of $*$-derivations $\left\{\delta_{j}: \mathcal{A} \longrightarrow \mathcal{A}\right\}_{j \in J}$ such that

$$
\delta_{j}(a) \longrightarrow \delta(a), \quad a \in \operatorname{Dom} \delta
$$

- also possesses this property, since all $N_{p}$ 's are closed $*$-ideals of $\mathcal{A}$ - see also [101] and [55].

At this point it seems a good idea to mention that the three theorems mentioned at the beginning of 1.2 (Hille-Yosida, Feller-Miyadera-Phillips and Lumer-Phillips Theorems) in the context of Banach spaces have generalizations to the locally convex' realm: [8, Theorem 4.2] and [8, Corollary 4.5] generalize [50,3.8 Generation Theorem (general case)] and [50, 3.5 Generation Theorem (contraction case)], respectively, to complete Hausdorff locally convex spaces. Likewise, [3, Theorem 3.14] is a locally convex version of Lumer-Phillips Theorem [50, 3.15 Theorem]. Since these more general theorems are going to be used in several different points of this manuscript, it seems reasonable to write their detailed statements here, for the sake of completeness: 
Feller-Miyadera-Phillips, Locally Convex Space Version [8, Theorem 4.2]: Let $(\mathcal{Y}, \Gamma)$ be a complete Hausdorff locally convex space. A necessary and sufficient condition for a closed linear operator $A$ to be the infinitesimal generator of a unique $\Gamma$ semigroup ${ }^{23}$ is that

1. the domain Dom $A$ of $A$ is dense in $\mathcal{Y}$;

2. A has the (KIP) with respect to $\Gamma$ and for each $p \in \Gamma$ the operator $A_{p}$ is closable in $\mathcal{Y}_{p}$

3. for each $p \in \Gamma$ there exist positive numbers $\sigma_{p}, M_{p}$ such that the resolvent $R\left(\lambda, \overline{A_{p}}\right)$ of $\overline{A_{p}}$ exists and satisfies the condition

$$
\left\|R\left(\lambda, \overline{A_{p}}\right)^{n}\right\|_{p} \leq M_{p}\left(\lambda-\sigma_{p}\right)^{-n}
$$

for all $\lambda>\sigma_{p}$ and $n \in \mathbb{N}$, where $\|\cdot\|_{p}$ denotes the usual operator norm on $\mathcal{L}\left(\mathcal{Y}_{p}\right){ }^{24}$

Hille-Yosida Theorem, Locally Convex Space Version [8, Corollary 4.5]: $A$ linear operator A generates a strongly continuous equicontinuous semigroup on a complete Hausdorff locally convex space if, and only if, it is the projective limit of generators of contraction semigroups on Banach spaces.

Lumer-Phillips Theorem, Locally Convex Space Version [3, Theorem 3.14]: Let $A$ be a $\Gamma$-dissipative, densely defined linear operator on a Hausdorff locally convex space $\mathcal{Y}$. Then, the following statements are equivalent:

1. The closure $\bar{A}$ of $A$ generates a strongly continuous equicontinuous semigroup on $\mathcal{Y}$.

2. Ran $(\lambda I-A)$ is dense in $\mathcal{Y}$ for some $\lambda>0$ (hence, for all $\lambda>0$ ).

Now, a very useful result involving the (KIP), and which will be invoked in Theorem 2.3 , is going to be proved:

Lemma 1.4.1: Let $\mathcal{X}$ be a sequentially complete Hausdorff locally convex space and let $T$ be the generator of an equicontinuous semigroup $t \longmapsto V(t)$ on $\mathcal{X}$. If $\Gamma$ is a fundamental system of seminorms for $\mathcal{X}$ with respect to which $T$ has the (KIP), is $\Gamma$-dissipative and $V$ is $\Gamma$-contractively equicontinuous (by what was observed at the beginning of this section, it is always possible to arrange such $\Gamma$ ), then $T_{p}$ is an infinitesimal pregenerator of a contraction semigroup on the Banach space $\mathcal{X}_{p}$, for all $p \in \Gamma$.

\footnotetext{
${ }^{23}$ See 1.1 for the definition of a $\Gamma$-semigroup.

${ }^{24}$ Actually, the closability requirement on $A_{p}$ is superfluous, and follows from Lemma 1.3.2 together with the third hypothesis.
} 
Proof of Lemma 1.4.1: Since $\mathcal{X}$ is sequentially complete, [78, Proposition 1.3] implies that $T$ is a densely defined linear operator. Also, being the generator of an equicontinuous semigroup, it satisfies

$$
\operatorname{Ran}(\lambda I-T)=\operatorname{Ran}(\lambda I-\bar{T})=\mathcal{X},
$$

for all $\lambda>0$, by [126, Corollary 1, page 241] (which was already invoked in Section 1.3). Hence, given $p \in \Gamma$, the induced linear operator $T_{p}$ on the quotient $\mathcal{X}_{p}$ is densely defined, dissipative and satisfies

$$
\left(\lambda I-T_{p}\right)\left[\pi_{p}[\operatorname{Dom} T]\right]=\mathcal{X} / N_{p}, \quad \lambda>0 .
$$

Therefore, $\overline{\operatorname{Ran}\left(\lambda I-T_{p}\right)}=\overline{\mathcal{X} / N_{p}}=\mathcal{X}_{p}$, showing that Ran $\left(\lambda I-T_{p}\right)$ is dense in $\mathcal{X}_{p}$, for all $p \in \Gamma$. By Lumer-Phillips Theorem on Banach spaces, $T_{p}$ is an infinitesimal pregenerator of a contraction semigroup on the Banach space $\mathcal{X}_{p}$.

Observation 1.4.1.1: If $\mathcal{X}_{p}=\overline{\mathcal{X} / N_{p}}=\mathcal{X} / N_{p}$, for all $p \in \Gamma$, then the stronger conclusion that $T_{p}$ is the generator, and not only a pregenerator of a contraction semigroup, may be obtained, for all $p \in \Gamma{ }^{25}$

Observation 1.4.1.2: A different version of Lemma 1.4.1 may be given, if $\mathcal{X}$ is assumed to be complete. If $T$ is only assumed to be a pregenerator of an equicontinuous group, then the same conclusion can be obtained: since $\bar{T}$ is a densely defined operator, so is T. Also, by the Lumer-Phillips Theorem for locally convex spaces [3, Theorem 3.14], Ran $(\lambda I-T)$ is a dense subspace of $\mathcal{X}$, so Ran $\left(\lambda I-T_{p}\right)$ is a dense subspace of $\mathcal{X}_{p}$, for all $p \in \Gamma$. Hence, the conclusion follows, just as in Lemma 1.4.1.

Observation 1.4.1.2, above, motivates the following definition:

Definition (Locally Convex Spaces with Complete Quotients): A Hausdorff locally convex space $(\mathcal{X}, \Gamma)$ having the property that its quotients $\mathcal{X} / N_{p}$ are already Banach spaces - in other words, $\mathcal{X} / N_{p}=\mathcal{X}_{p}$, for all $p \in \Gamma$ - will be said to have complete quotients.

If $\mathcal{Y}$ is a Banach space and $T$ is a linear operator defined on $\mathcal{Y}$, then a vector $y \in \mathcal{Y}$ is called analytic for $\boldsymbol{T}$ if

$$
y \in C^{\infty}(T):=\bigcap_{n=1}^{+\infty} \operatorname{Dom} T^{n}
$$

and there exists $r_{y}>0$ such that

$$
\sum_{n \geq 0} \frac{\left\|T^{n}(y)\right\|}{n !}|u|^{n}<\infty, \quad|u|<r_{y} .
$$

\footnotetext{
${ }^{25}$ This is the case, for example, when $\mathcal{X} \equiv \mathcal{A}$ is a locally $\mathrm{C}^{*}$-algebra - see Chapter 3.
} 
A very useful theorem which deals with the relation between infinitesimal generators and analytic vectors is the following:

Isometry Group Generation Theorem - Analytic Vectors, Banach Space Version [111, Theorem 1, Theorem 3]: Let $\mathcal{Y}$ be a Banach space and $A$ an operator on $\mathcal{Y}$. Suppose that

1. the set of analytic vectors of $A$ in dense in $\mathcal{Y}$;

2. for all $\lambda \in \mathbb{R} \backslash\{0\}$ and all $y \in \operatorname{Dom} A$,

$$
\|(\lambda I-A) y\| \geq|\lambda|\|y\|
$$

or, in other words, $A$ is conservative.

Then, A has a closure generating a strongly continuous one-parameter group of isometries.

Reference [111] actually establishes sufficient conditions on an operator in order for it to be a pregenerator of a general strongly continuous one-parameter group (not necessarily implemented by isometries). However, for the purposes of this thesis, it is this version of the theorem which interests the most.

The next task will be to define projective analytic vectors on locally convex spaces, so that some useful theorems become available:

Definition 1.4.2 (Projective Analytic Vectors): Let $(\mathcal{X}, \tau)$ be a locally convex space with a fundamental system of seminorms $\Gamma$ and $T$ a linear operator defined on $\mathcal{X}$. An element $x \in \mathcal{X}$ is called a $\tau$-projective analytic vector for $T$ if

$$
x \in C^{\infty}(T):=\bigcap_{n=1}^{+\infty} \operatorname{Dom} T^{n}
$$

and, for every $p \in \Gamma$, there exists $r_{x, p}>0$ such that

$$
\sum_{n \geq 0} \frac{p\left(T^{n}(x)\right)}{n !}|u|^{n}<\infty, \quad|u|<r_{x, p} .
$$

Note that, for this definition to make sense, it is necessary to show that it does not depend on the choice of the particular system of seminorms: if $x$ is $\tau$-projective analytic with respect to $\Gamma$ and $\Gamma^{\prime}$ is another saturated family of seminorms generating the topology of $\mathcal{X}$ then, for each $q^{\prime} \in \Gamma^{\prime}$, there exists $C_{q^{\prime}}>0$ and $q \in \Gamma$ such that $q^{\prime}(y) \leq C_{q^{\prime}} q(y)$, for all 
$y \in \mathcal{X}$. Therefore, making $r_{x, q^{\prime}}:=r_{x, q}$, one obtains for every $u \in \mathbb{C}$ satisfying $|u|<r_{x, q^{\prime}}$ that

$$
\sum_{n \geq 0} \frac{q^{\prime}\left(T^{n}(x)\right)}{n !}|u|^{n} \leq C_{q^{\prime}} \sum_{n \geq 0} \frac{q\left(T^{n}(x)\right)}{n !}|u|^{n}<\infty .
$$

By symmetry, the assertion is proved. This motivates the use of the notation " $\tau$-projective analytic" to indicate that the projective analytic vector in question is related to the topology $\tau$. Sometimes it will be necessary to make explicit which is the topology under consideration to talk about projective analytic vectors, since in some occasions it will be necessary to deal with more than one topology at once - see Theorem 2.9 for a concrete illustration of this situation. When there is no danger of confusion, the symbol $\tau$ will be omitted. The subspace formed by all of the projective analytic vectors for $T$ is going to be denoted by $C_{\leftarrow}^{\omega}(T)$. The prefix "projective" stands for the fact that $C_{\leftarrow}^{\omega}(T)$ can be seen as a dense subspace of the projective $\operatorname{limit} \varliminf_{\lim } \pi_{p}\left[C_{\leftarrow}^{\omega}(T)\right]$ via the canonical map $x \longmapsto\left([x]_{p}\right)_{p \in \Gamma}$ and, if $T$ has the (KIP) with respect to $\Gamma$, then $\pi_{p}\left[C_{\leftarrow}^{\omega}(T)\right]$ consists entirely of analytic vectors for $T_{p}$, for every $p \in \Gamma$ (see Section 1.7 for the definition of a projective limit of locally convex spaces, and Lemma 1.7.1 for the denseness claim). Note that the projective limit is well-defined, since the family $\left\{\pi_{p}\left[C_{\leftarrow}^{\omega}(T)\right]\right\}_{p \in \Gamma}$ gives rise to a canonical projective system.

Differently of what is required from the usual definition of analytic vectors, no uniformity in $\boldsymbol{p}$ is asked in the above definition. Indeed, using the definition of analytic vectors given before, it is possible to adapt the proof of [65, Theorem 2, page 209] and conclude that for every analytic vector $x \in \mathcal{X}$ for $T$ there exists $r_{x}>0$ such that, whenever $|u|<r_{x}$, the series

$$
\sum_{n=0}^{+\infty} \frac{p\left(T^{n}(x)\right)}{n !}|u|^{n}
$$

is convergent, for every $p \in \Gamma$. Hence, the definition just given is weaker that the usual one, so the subspace of $\tau$-analytic vectors $C^{\omega}(T)$ satisfies $C^{\omega}(T) \subseteq C_{\leftarrow}^{\omega}(T)$.

The next theorem, which is a "locally convex version" of [111, Theorem 3], will play an important role in Theorems 2.9 and 2.12:

Lemma 1.4.3: Let $(\mathcal{X}, \Gamma)$ be a complete Hausdorff locally convex space and let $T$ be a $\Gamma$-conservative linear operator on $\mathcal{X}$ (hence, it is closable, by Corollary 1.3.3) having the (KIP) with respect to $\Gamma$. If $T$ has a dense set of projective analytic vectors, then $\bar{T}$ is the generator of a $\Gamma$-isometrically equicontinuous group.

Proof of Lemma 1.4.3: For each $p \in \Gamma$, the densely defined linear operator $T_{p}$ induced on the quotient $\mathcal{X} / N_{p}$ possesses a dense subspace $\pi_{p}\left[C_{\leftarrow}^{\omega}(T)\right]$ of analytic vectors and is conservative. Therefore, by [111, Theorem 2], $T_{p}$ is an infinitesimal pregenerator of a group of isometries $t \longmapsto V_{p}(t)$. This implies $\operatorname{Ran}\left(\lambda I-T_{p}\right)$ is dense in $\mathcal{X}_{p}$ for all 
$\lambda \in \mathbb{R} \backslash\{0\}$, by Lumer-Phillips Theorem. Fix $\lambda \in \mathbb{R} \backslash\{0\}$. The idea, now, is to prove $\operatorname{Ran}(\lambda I-T)$ is dense in $\mathcal{X}$. So fix $y \in \mathcal{X}, \epsilon>0, p \in \Gamma$ and

$$
V:=\{x \in \mathcal{X}: p(x-y)<\epsilon\}
$$

an open neighborhood of $y$. Denseness of $\operatorname{Ran}\left(\lambda I-T_{p}\right)$ in $\mathcal{X}_{p}$ implies the existence of $x_{0} \in \operatorname{Dom} T$ such that $p\left((\lambda I-T)\left(x_{0}\right)-y\right)<\epsilon$. Hence, $V \cap \operatorname{Ran}(\lambda I-T) \neq \emptyset$. By the arbitrariness of $p$, it follows that $y$ must belong to $\overline{\operatorname{Ran}(\lambda I-T)}$, so $\overline{\operatorname{Ran}(\lambda I-T)}=\mathcal{X}, T$ is closable and $\bar{T}$ must be the infinitesimal generator of an equicontinuous group $t \longmapsto V(t)$, by $[3$, Proposition 3.13$]$ and by the Lumer-Phillips Theorem for locally convex spaces $[3$, Theorem 3.14]. ${ }^{26}$

To see that $\bar{T}$ is actually the generator of a $\Gamma$-isometrically equicontinuous group, first note that formula (7) on [126, page 248] says that

$$
V(t) x=\lim _{n \rightarrow+\infty} \exp \left(t \bar{T}\left(I-\frac{1}{n} \bar{T}\right)^{-1}\right) x, \quad x \in \mathcal{X}, t \in[0,+\infty) .
$$

Since $\bar{T}$ is $\Gamma$-dissipative,

$$
p\left((I-\lambda \bar{T})^{-1}(x)\right) \leq p(x), \quad p \in \Gamma, \lambda>0,
$$

so the identity

$$
\bar{T}\left(I-\frac{1}{n} \bar{T}\right)^{-1}=n\left(\left(I-\frac{1}{n} \bar{T}\right)^{-1}-I\right)
$$

shows, for every fixed $t \geq 0, p \in \Gamma$ and $x \in \mathcal{X}$ satisfying $p(x) \leq 1$, that

$$
\begin{gathered}
p\left(\exp \left(t \bar{T}(I-(1 / n) \bar{T})^{-1}\right) x\right)=p\left(\exp \left(t n(I-(1 / n) \bar{T})^{-1}-t n\right) x\right) \\
=p\left(\exp \left(t n(I-(1 / n) \bar{T})^{-1}\right) \exp (-t n) x\right) \leq \exp (t n) p(x) \exp (-t n) \leq 1 .
\end{gathered}
$$

Hence,

$$
p(V(t) x) \leq p(x)
$$

for every $t \geq 0, p \in \Gamma$ and $x \in \mathcal{X}$. But $-\bar{T}$ also generates an equicontinuous semigroup (more precisely, it generates the semigroup $V_{-}: t \longmapsto V(-t)$ ), so formula

$$
V(-t) x=V_{-}(t) x=\lim _{n \rightarrow+\infty} \exp \left(-t \bar{T}\left(I+\frac{1}{n} \bar{T}\right)^{-1}\right) x, \quad x \in \mathcal{X}, t \in[0,+\infty),
$$

\footnotetext{
${ }^{26}[3$, Proposition 3.13]: Let $A$ be a $\Gamma$-dissipative linear operator on a Hausdorff locally convex space $\mathcal{Y}$. If $A$ is densely defined, then $A$ is closable and its closure is also a $\Gamma$-dissipative operator on $\mathcal{Y}$. Moreover, if $\mathcal{Y}$ is complete, then $\operatorname{Ran}(\lambda I-\bar{A})=\overline{\operatorname{Ran}(\lambda I-A)}$ for all $\lambda>0$.
} 
is also valid. Therefore, for every fixed $t \geq 0, p \in \Gamma$ and $x \in \mathcal{X}$ satisfying $p(x) \leq 1$,

$$
\begin{aligned}
& p\left(\exp \left(-t \bar{T}(I+(1 / n) \bar{T})^{-1}\right) x\right)=p\left(\exp \left(t n(I+(1 / n) \bar{T})^{-1}-t n\right) x\right) \\
= & p\left(\exp \left(t n(I+(1 / n) \bar{T})^{-1}\right) \exp (-t n) x\right) \leq \exp (-t n) \exp (t n p(x)) \leq 1,
\end{aligned}
$$

so taking the limit $n \longrightarrow+\infty$ on both sides of this inequality shows $p(V(t) x) \leq p(x)$, for all $t \leq 0, p \in \Gamma$ and $x \in \mathcal{X}$. This proves $p(V(t) x) \leq p(x)$, whenever $t \in \mathbb{R}, p \in \Gamma$ and $x \in \mathcal{X}$. But this also shows that $p(x)=p(V(-t) V(t) x) \leq p(V(t) x)$, for all $t \in \mathbb{R}, p \in \Gamma$ and $x \in \mathcal{X}$, so $V$ is a $\Gamma$-isometrically equicontinuous group.

Actually, a much more general version of Lemma 1.4.3 is available: ${ }^{27}$

Theorem 1.4.4: Let $(\mathcal{X}, \Gamma)$ be a complete Hausdorff locally convex space and $T$ a linear operator on $\mathcal{X}$ having the (KIP) with respect to $\Gamma$. Suppose that $T$ has a dense set of projective analytic vectors and that, for each $p \in \Gamma$, there exist numbers $\sigma_{p} \geq 0, M_{p}>0$, such that

$$
p\left((\lambda-T)^{n} x\right) \geq M_{p}^{-1}\left(|\lambda|-\sigma_{p}\right)^{n} p(x), \quad x \in \operatorname{Dom}^{n},
$$

for all $|\lambda|>\sigma_{p}$ and $n \in \mathbb{N}, n \geq 1$. Then, $T$ is closable and $\bar{T}$ is the generator of a $\Gamma$-group satisfying $p(V(t) x) \leq M_{p} e^{\sigma_{p}|t|} p(x)$, for all $p \in \Gamma, x \in \mathcal{X}$ and $t \in \mathbb{R}$.

Proof of Theorem 1.4.4: For each $p \in \Gamma$, the densely defined linear operator $T_{p}$ induced on the quotient $\mathcal{X} / N_{p}$ possesses a dense subspace $\pi_{p}\left[C_{\leftarrow}^{\omega}(T)\right]$ of analytic vectors. Hence, by [111, Theorem 1, Theorem 2], $T_{p}$ is closable and $\overline{T_{p}}$ is the generator of a strongly continuous one-parameter group on $\mathcal{X}_{p}$, so the projective limit $\tilde{T}$ of the family $\left\{\overline{T_{p}}\right\}_{p \in \Gamma}$ is the generator of a $\Gamma$-group $t \longmapsto V(t)$ such that $T \subset \tilde{T}$, by [8, Theorem 2.5] (adapted to one-parameter groups). In particular, this shows that $T$ is closable and

$$
\bar{T} \subset \tilde{T}
$$

since $\tilde{T}$ is closed. Therefore, since $V$ leaves the dense subspace $C_{\leftarrow}^{\omega}(T) \subseteq C^{\infty}(\tilde{T})$ invariant, it follows from Lemma 0, above, that it is a core for $\tilde{T}$, so

$$
\tilde{T}=\overline{\left.\tilde{T}\right|_{C_{\leftarrow}^{\omega}(T)}}=\overline{\left.T\right|_{C_{\leftarrow}^{\omega}(T)}} \subset \bar{T} .
$$

This establishes the result.

\footnotetext{
${ }^{27}$ The remainder of this section uses a lot of the content exposed in Section 1.7, so the reader may want to take a look at that section before proceeding. It should also be emphasized that the theorems to follow, until the end of this section, are not required to prove the exponentiation theorems of Sections 2 and 3.
} 
Corollary 1.4.5: Let $(\mathcal{X}, \Gamma)$ be a complete Hausdorff locally convex space and $T$ a linear operator on $\mathcal{X}$ having the (KIP) with respect to $\Gamma$. Suppose that $T$ has a dense set of analytic vectors and that, for each $p \in \Gamma$, there exist numbers $\sigma_{p} \geq 0, M_{p}>0$, such that

$$
p\left((\lambda-T)^{n} x\right) \geq M_{p}^{-1}\left(|\lambda|-\sigma_{p}\right)^{n} p(x), \quad x \in \operatorname{Dom}^{n},
$$

for all $|\lambda|>\sigma_{p}$ and $n \in \mathbb{N}, n \geq 1$. Then, $T$ is closable and $\bar{T}$ is the generator of a $\Gamma$-group satisfying $p(V(t) x) \leq M_{p} e^{\sigma_{p}|t|} p(x)$, for all $p \in \Gamma, x \in \mathcal{X}$ and $t \in \mathbb{R}$.

Proof of Corollary 1.4.5: Follows at once from Theorem 1, since $C^{\omega}(T) \subseteq C_{\leftarrow}^{\omega}(T)$.

Actually, a converse statement for Theorem 1.4.4 is also true:

Theorem 1.4.6: Let $(\mathcal{X}, \Gamma)$ be a complete Hausdorff locally convex space and $T$ a linear operator on $\mathcal{X}$ which is the generator of a $\Gamma$-group. Then, $T$ has a dense set of projective analytic vectors and there exist numbers $\sigma_{p} \geq 0, M_{p}>0$, such that

$$
p\left((\lambda-T)^{n} x\right) \geq M_{p}^{-1}\left(|\lambda|-\sigma_{p}\right)^{n} p(x), \quad x \in \operatorname{Dom}^{n},
$$

for all $|\lambda|>\sigma_{p}$ and $n \in \mathbb{N}, n \geq 1$.

Proof of Theorem 1.4.6: By [8, Theorem 4.2], there exists a projective family $\left\{T_{p}\right\}_{p \in \Gamma}$ of linear operators such that $\overline{T_{p}}$ is the generator of a strongly continuous oneparameter group on $\mathcal{X}_{p}$, for each $p \in \Gamma$, and $T=\lim \overline{T_{p}}$. Therefore, applying [95, Theorem 3.1] and [94, Theorem 4], together with [102, Theorem 1.4], to the one-dimensional Lie group $\mathbb{R}$, it follows that $\overline{T_{p}^{2}}$ is the generator of a strongly continuous one-parameter semigroup $t \longmapsto S_{p}(t)$ on $\mathcal{X}_{p}$ satisfying

$$
S_{p}(t)\left[\mathcal{X}_{p}\right] \subseteq C^{\omega}\left(\overline{T_{p}}\right), \quad t>0
$$

for each $p \in \Gamma{ }^{28}$ Hence,

$$
\bigcup_{t>0} S_{p}(t)\left[\mathcal{X}_{p}\right] \subseteq C^{\omega}\left(\overline{T_{p}}\right), \quad p \in \Gamma, t>0
$$

Repeating the argument made in the proof of [8, Theorem 4.2] on formula

$$
S_{p}(t) x_{p}=\lim _{\lambda \rightarrow+\infty} \exp (-\lambda t) \sum_{k=0}^{+\infty} \frac{(\lambda t)^{k}\left[\lambda \mathrm{R}\left(\lambda, \overline{T_{p}^{2}}\right)\right]^{k}}{k !}\left(x_{p}\right), \quad p \in \Gamma, t \geq 0, x_{p} \in \mathcal{X}_{p}
$$

\footnotetext{
${ }^{28}$ See also [25, Corollary 2.6].
} 
(this formula may also be found in $\left[68,(11.7 .2)\right.$, page 352]) one sees that $\left\{S_{p}(t)\right\}_{p \in \Gamma}$ is a projective family of linear operators (see Section 1.7, for this definition), for each fixed $t>0$. Hence, the projective limit semigroup

$$
S: t \longmapsto S(t):=\lim _{\longleftarrow} S_{p}(t), \quad t \geq 0,
$$

on $\lim _{\longleftarrow} \mathcal{X}_{p}$ is well-defined and satisfies

$$
\bigcup_{t>0} S(t)[\mathcal{X}] \subseteq C_{\leftarrow}^{\omega}(T)
$$

It is also strongly continuous [91, Lemma 7b), page 26], so that the union above is dense in $\mathcal{X}$. This shows the density of $C_{\leftarrow}^{\omega}(T)$ in $\mathcal{X}$.

The claimed estimates follow at once from [8, Theorem 4.2].

Still under the hypotheses of the theorem just proved, it is possible to show that $\overline{T^{2}}$ is the generator of a $\Gamma$-semigroup: since $\overline{T^{2}} \subset \lim \overline{T_{p}^{2}}$ and $C^{\infty}(T)$ is left invariant by the operators $S(t)$ (in view of $S_{p}(t)\left[\mathcal{X}_{p}\right] \subseteq C^{\infty}\left(\overline{T_{p}}\right)$, for all $t>0$ and $p \in \Gamma$ ), an application of Corollary 1.2.4.2, above, yields $\overline{T^{2}}=\lim _{\longleftarrow} \overline{T_{p}^{2}}$, since Theorem 1.4.6 shows that $C^{\infty}(T)$ is dense in $\mathcal{X}$. Also, Theorem 1.4.6 proves that the subspace $\mathcal{X}_{0}:=\{S(t) x: x \in \mathcal{X}, t>0\}$ is dense in $\mathcal{X}$, so another application of Corollary 1.2.4.2 shows that $\mathcal{X}_{0}$ is a core for $\overline{T^{2}}$. Summarizing:

Corollary 1.4.7: Let $(\mathcal{X}, \Gamma)$ be a complete Hausdorff locally convex space and $T$ a linear operator on $\mathcal{X}$ which is the generator of a $\Gamma$-group. Then, $\overline{T^{2}}$ is the generator of a $\Gamma$-semigroup and $\mathcal{X}_{0}:=\{S(t) x: x \in \mathcal{X}, t>0\}$ is a dense subspace of $\mathcal{X}$ which is a core for $\overline{T^{2}}$. Since $\mathcal{X}_{0} \subseteq C_{\leftarrow}^{\omega}(T) \subseteq C^{\infty}(T)$, the dense subspaces $C^{\infty}(T)$ and $C_{\leftarrow}^{\omega}(T)$ are also cores for $\overline{T^{2}}$.

Therefore, the following theorem holds:

Theorem 1.4.8: Let $(\mathcal{X}, \Gamma)$ be a complete Hausdorff locally convex space and $T$ a closed linear operator on $\mathcal{X}$. Then, $T$ is the generator of a $\Gamma$-group if, and only if, the following two conditions are satisfied:

1. $T$ has the (KIP) with respect to $\Gamma$ and, for each $p \in \Gamma$, there exist numbers $\sigma_{p} \geq 0$, $M_{p}>0$, such that

$$
p\left((\lambda-T)^{n} x\right) \geq M_{p}^{-1}\left(|\lambda|-\sigma_{p}\right)^{n} p(x), \quad x \in \operatorname{Dom}^{n},
$$

for all $|\lambda|>\sigma_{p}$ and $n \in \mathbb{N}, n \geq 1$, and 
2. $T$ has a dense set of projective analytic vectors.

In this case, (a) if $t \longmapsto V(t)$ is the strongly continuous one-parameter group generated by $T$, then $p(V(t) x) \leq M_{p} e^{\sigma_{p}|t|} p(x)$, for all $p \in \Gamma, x \in \mathcal{X}, t \in \mathbb{R}$ and (b) $\overline{T^{2}}$ generates a $\Gamma$-semigroup $t \longmapsto S(t)$ such that the dense subspaces $\mathcal{X}_{0}:=\{S(t) x: x \in \mathcal{X}, t>0\}, C^{\infty}(T)$ and $C_{\leftarrow}^{\omega}(T)$ are cores for $\overline{T^{2}}$.

The theorems above apply directly for equicontinuous one-parameter groups, with the appropriate choice of $\Gamma$, making $M_{p}=1$ and $\sigma_{p}=0$, for every $p \in \Gamma$. Together with $[3$, Theorem 3.14], they give the following useful corollary:

Corollary 1.4.9: Let $(\mathcal{X}, \tau)$ be a complete Hausdorff locally convex space and $T$ a linear operator on $\mathcal{X}$. Then,

1. If $T$ is closed, $T$ will be the generator of an equicontinuous group if, and only if, it has a dense set of projective analytic vectors and there is a fundamental system of seminorms $\Gamma$ for which it is $\Gamma$-conservative.

2. If $T$ is a conservative operator having a dense set of projective analytic vectors, then $\bar{T}$ is the generator of an equicontinuous one-parameter group and Ran $(\lambda I-T)$ is dense in $\mathcal{X}$, for every nonzero $\lambda \in \mathbb{R}$; if $T$ is a $\Gamma$-conservative operator for which Ran $(\lambda I-T)$ is dense in $\mathcal{X}$, for some nonzero $\lambda \in \mathbb{R}$, then $T$ is closable and $\bar{T}$ has a dense set of projective analytic vectors.

Proof of Corollary 1.4.9: (1) Follows directly from Theorem 1.4.8.

(2) If $T$ is a conservative operator having a dense set of projective analytic vectors, then Theorem 1.4.4 says that it is closable and that $\bar{T}$ is the generator of an equicontinuous one-parameter group. Hence, by [3, Theorem 3.14] (adapted for one-parameter groups), $\operatorname{Ran}(\lambda I-T)$ is dense in $\mathcal{X}$, for every nonzero $\lambda \in \mathbb{R}$. If, however, $T$ is a $\Gamma$-conservative operator for which $\operatorname{Ran}(\lambda I-T)$ is dense in $\mathcal{X}$, for some nonzero $\lambda \in \mathbb{R}$, then $[3$, Theorem 3.14] for groups shows that $T$ is closable and that $\bar{T}$ is the generator of a $\Gamma$-isometrically equicontinuous group. Therefore, by Theorem 1.4.6, $\bar{T}$ has a dense set of projective analytic vectors.

Example 1.4.10: Let $\mathcal{X}$ be a Banach space, $G$ a real finite-dimensional Lie group of dimension $d$ with Lie algebra $\mathfrak{g}$ and $V: G \longrightarrow \mathcal{L}(\mathcal{X})$ a strongly continuous representation of $G$ on $\mathcal{X}$. Fix an ordered basis $\mathcal{B}:=\left(X_{k}\right)_{1 \leq k \leq d}$ for $\mathfrak{g}$. To begin with, fix a norm $\|\cdot\|$ on $\mathcal{X}$, equivalent to the original one, having the property that there exists $\beta \in \mathbb{R}$ such that, for each operator $\partial V\left(X_{k}\right), 1 \leq k \leq d$, and every $\lambda \in \mathbb{C}$ satisfying $|\operatorname{Re} \lambda|>\beta$, one has

$$
\left\|\left(\lambda I-d V\left(X_{k}\right)\right)^{m} x\right\| \geq(|\operatorname{Re} \lambda|-\beta)^{m}\|x\|, \quad x \in C^{\infty}(V), m \in \mathbb{N},
$$




\subsection{The Kernel Invariance Property (KIP),}

just as done in [59, Theorem 3.1] (this follows from a rescaling argument [50, page 78] together with an application of the Feller-Miyadera-Phillips Theorem for one-parameter groups), and equip $C^{\infty}(V)$ with the projective $\mathrm{C}^{\infty}$-topology on $C^{\infty}(V)$.

As an application of the above theorems, it will be shown that $V_{\infty}: g \longmapsto V_{\infty}(g)$ is a strongly continuous representation of $G$ on $\left(C^{\infty}(V), \tau_{\infty}\right)$, with each $\partial V(X)$ being the generator of the $\Gamma_{\infty}$-group $t \longmapsto V_{\infty}(\exp t X)$ - note that $\partial V(X)$ is a continuous operator on $\left(C^{\infty}(V), \tau_{\infty}\right)$. Theorem 2 of [65] shows that, given $X \in \mathfrak{g}$ and $x \in C^{\omega}(V)$, there exists $r_{x}>0$ such that the series

$$
\sum_{m=0}^{+\infty} \frac{\left\|d V\left(X^{m}\right) x\right\|}{m !}|u|^{m},
$$

converges, if $|u|<r_{x}$. Since $C^{\omega}(V)$ is left invariant by the operators $d V(X), X \in \mathfrak{g}$ (see [65, page 209]), it is possible to iterate the calculations of Observations 1 and 2 of [59, Theorem 3.1] to obtain, respectively: (a) for each fixed $X \in \mathfrak{g}, n \in \mathbb{N}$ and $x \in C^{\omega}(V)$, the series

$$
\sum_{m=0}^{+\infty} \frac{\left\|d V\left(X^{m}\right) x\right\|_{n}}{m !}|u|^{m},
$$

converges for sufficiently small $|u|$ and, (b) for every $n \in \mathbb{N}, x \in C^{\infty}(V)$ and $\lambda \in \mathbb{C}$ satisfying $|\operatorname{Re} \lambda|>l_{n}(k):=\beta+n \tau_{k}$, one has

$$
\left\|\left(\lambda I-d V\left(X_{k}\right)\right)^{m} x\right\|_{n} \geq\left(|\operatorname{Re} \lambda|-l_{n}(k)\right)^{m}\|x\|_{n}, \quad m \in \mathbb{N},
$$

where $\tau_{k} \equiv \tau\left(X_{k}\right)$ is the operator norm of ad $\partial V\left(X_{k}\right):=\left[\partial V\left(X_{k}\right), \cdot\right]$, when seen as a linear operator on $\left(\partial V[\mathfrak{g}],\|\cdot\|_{1}\right)$, with $\left\|\sum_{j=1}^{d} c_{j} \partial V\left(X_{j}\right)\right\|_{1}:=\sum_{j=1}^{d}\left|c_{j}\right|$. In particular, (a) shows that $C^{\omega}(V) \subseteq \cap_{k=1}^{d} C_{\leftarrow}^{\omega}\left(d V\left(X_{k}\right)\right)$. Also, note that $\cap_{k=1}^{d} C_{\leftarrow}^{\omega}\left(d V\left(X_{k}\right)\right) \subseteq C^{\infty}(V)$. By [102, Theorem 1.3], $C^{\omega}(V)$ is $\tau_{\infty}$-dense in $C^{\infty}(V)$, since it is a $\tau$-dense subspace of $\mathcal{X}[94$, Theorem 4] which satisfies $V(g)\left[C^{\omega}(V)\right] \subseteq C^{\omega}(V)$, for all $g \in G$. Hence, it follows that $C_{\leftarrow}^{\omega}\left(d V\left(X_{k}\right)\right)$ is $\tau_{\infty}$-dense in $C^{\infty}(V)=$ Dom $\partial V\left(X_{k}\right)$, for all $1 \leq k \leq d$. The operators $\partial V\left(X_{k}\right), 1 \leq k \leq d$, also have the (KIP) with respect to $\Gamma_{\infty}$ which, combined with (b), show that their closures ${\overline{\partial V\left(X_{k}\right)}}^{\infty}$ with respect to $\tau_{\infty}$ are generators of $\Gamma_{\infty}$-groups, by Theorem 1.4.4. Since they are all $\tau_{\infty}$-continuous, every $\partial V\left(X_{k}\right)={\overline{\partial V\left(X_{k}\right)}}^{\infty}$ is the generator of a $\Gamma_{\infty}$-group $V_{k}: t \longmapsto V_{k}(t)$ on $C^{\infty}(V)$. To see that $V_{k}(t)=V_{\infty}\left(\exp t X_{k}\right)$, for every $t \in \mathbb{R}$ and $1 \leq k \leq d$, just extend both $V_{k}$ and $V_{\infty}$ to strongly continuous one-parameter groups on all of $\mathcal{X}$, use the fact that $\tau_{\infty}$ is finer than $\tau$ and that two strongly continuous one-parameter groups on a Banach space having the same infinitesimal generator must be equal. Now, there exist $d$ real-valued analytic functions $\left\{t_{k}\right\}_{1 \leq k \leq d}$ defined on a relatively compact open neighborhood $\Omega$ of the identity of $G$ such that $g \longmapsto\left(t_{k}(g)\right)_{1 \leq k \leq d}$ maps $\Omega$ diffeomorphically onto a neighborhood of the origin of $\mathbb{R}^{d}$, with

$$
g=\exp \left(t_{1}(g) X_{1}\right) \ldots \exp \left(t_{k}(g) X_{k}\right) \ldots \exp \left(t_{d}(g) X_{d}\right), \quad g \in \Omega,
$$


SO

$$
V_{\infty}(g)=V\left(\exp \left(t_{1}(g) X_{1}\right)\right) \ldots V\left(\exp \left(t_{k}(g) X_{k}\right)\right) \ldots V\left(\exp \left(t_{d}(g) X_{d}\right)\right)
$$

on this neighborhood, which establishes the strong continuity of $V_{\infty}$ with respect to $\tau_{\infty}$. Choosing an adequate norm for each fixed $X \in \mathfrak{g}$, just like it was done with the basis elements, and repeating the above reasoning, one sees that each $\partial V(X)$ is the generator of a $\Gamma_{\infty}$-group such that, for every $n \in \mathbb{N}, x \in C^{\infty}(V)$ and $\lambda \in \mathbb{C}$ satisfying $|\operatorname{Re} \lambda|>l_{n}(X):=$ $\beta(X)+n \tau(X)$, one has

$$
\left\|(\lambda I-\partial V(X))^{m} x\right\|_{n} \geq \frac{\left(|\operatorname{Re} \lambda|-l_{n}(X)\right)^{m}}{M}\|x\|_{n}, \quad m \in \mathbb{N},
$$

for certain numbers $\beta(X) \in \mathbb{R}$ and $M>0$, where $\tau(X)$ is the operator norm of ad $\partial V(X):=$ $[\partial V(X), \cdot]$, when seen as a linear operator on $\left(\partial V[\mathfrak{g}],\|\cdot\|_{1}\right) \cdot{ }^{29}$ Summarizing:

Theorem: If $(\mathcal{X}, \tau)$ is a Banach space and $V: G \longrightarrow \mathcal{L}(\mathcal{X})$ is a strongly continuous representation, then it restricts to a strongly continuous representation $V: G \longrightarrow \mathcal{L}\left(C^{\infty}(V)\right)$ on $\left(C^{\infty}(V), \tau_{\infty}\right)$ which is implemented by (one-parameter) $\Gamma_{\infty}$-groups. If $\tau(X)=0$, then $t \longmapsto V(\exp t X)$ is of bounded type.

To illustrate this situation, consider the Schwartz function space $\mathcal{S}\left(\mathbb{R}^{n}\right)$ as a subspace of $L^{2}\left(\mathbb{R}^{n}\right)$ and equip it with the family $\Gamma$ of seminorms $f \longmapsto\left\|x^{\alpha} \partial^{\beta} f\right\|_{2}$, where $\|\cdot\|_{2}$ denotes the $\mathrm{L}^{2}$-norm and $\alpha, \beta \in \mathbb{N}^{n}$ are multi-indices. Also, consider the Heisenberg group $H_{2 n+1}(\mathbb{R})$ defined at the beginning of Chapter 2 , along with the strongly continuous unitary representation $U$ of $H_{2 n+1}(\mathbb{R})$ defined by

$$
U_{\mathbf{a}, \mathbf{b}, c}:=U\left[\begin{array}{ccc}
1 & \mathbf{a} & c \\
0 & I_{n} & \mathbf{b} \\
0 & 0 & 1
\end{array}\right]: f \longmapsto\left(\mathcal{M}_{c} \circ M_{\mathbf{b}} \circ T_{\mathbf{a}}\right)(f),
$$

where $\left(\mathcal{M}_{c} \circ M_{\mathbf{b}} \circ T_{\mathbf{a}}\right)(f)(x)=e^{i c} e^{i\langle\mathbf{b}, x\rangle} f(x+\mathbf{a})$. Then, by the calculations performed in Chapter 2 , its Lie algebra is sent onto the $(2 n+1)$-dimensional real Lie algebra

$$
\mathcal{L}:=\operatorname{span}_{\mathbb{R}}\left\{\left.\partial_{k}\right|_{\mathcal{S}\left(\mathbb{R}^{n}\right)},\left.i x_{k}\right|_{\mathcal{S}\left(\mathbb{R}^{n}\right)},\left.i\right|_{\mathcal{S}\left(\mathbb{R}^{n}\right)}: 1 \leq k \leq n\right\}
$$

via the Lie algebra representation $\partial U$, since $C^{\infty}(U)=\mathcal{S}\left(\mathbb{R}^{n}\right)$, and it is a realization of the Canonical Commutation Relations (CCR) by unbounded operators on $L^{2}\left(\mathbb{R}^{n}\right)$. Therefore, the calculations above show, in particular, that the linear operators $c_{0} i+\sum_{k=1}^{n} c_{k} \partial_{k}+$ $\sum_{k=1}^{n} d_{k} x_{k}, c_{k}, d_{k} \in \mathbb{R}$, all generate $\Gamma_{\infty}$-groups on $\mathcal{S}\left(\mathbb{R}^{n}\right)$, since $U$ is unitary, thus complementing some of the examples given in [8]. Moreover, their squares generate $\Gamma_{\infty}$-semigroups

\footnotetext{
${ }^{29}$ If $\|\cdot\|^{\prime}$ and $\|\cdot\|^{\prime \prime}$ are two equivalent norms on $\mathcal{X}$ satisfying $M_{1}\|\cdot\|^{\prime \prime} \leq\|\cdot\|^{\prime} \leq M_{2}\|\cdot\|^{\prime \prime}$, for certain $M_{1}, M_{2}>0$, then $M_{1}\|\cdot\|_{n}^{\prime \prime} \leq\|\cdot\|_{n}^{\prime} \leq M_{2}\|\cdot\|_{n}^{\prime \prime}$, for every $n \in \mathbb{N}$. The constant $M$, above, appears as a consequence of this reasoning.
} 
on $\mathcal{S}\left(\mathbb{R}^{n}\right)$.

For yet another example, consider the torus $\mathbb{T}^{n}:=\mathbb{R}^{n} /(2 \pi \mathbb{Z})^{n}$, where it will be used the same letter $x$ to denote both an element $x$ of $\mathbb{R}^{n}$ and its class $[x] \in \mathbb{T}^{n}$. For each $y \in \mathbb{T}^{n}$, let $T_{y}$ denote the unitary operator on $L^{2}\left(\mathbb{T}^{n}\right)$ defined by $\left(T_{y} u\right)(x)=u(x-y)$. For each $j \in \mathbb{Z}^{n}$, let $e_{j} \in C^{\infty}\left(\mathbb{T}^{n}\right)$ be defined by $e_{j}(x)=e^{i\langle j, x\rangle}$. Then, by [33, Theorem 2], ${ }^{30}$ a bounded operator $A \in \mathcal{L}\left(L^{2}\left(\mathbb{T}^{n}\right)\right)$ is such that the map $\mathbb{T}^{n} \ni y \longmapsto T_{y} A T_{-y}$ is smooth with respect to the norm topology of $\mathcal{L}\left(L^{2}\left(\mathbb{T}^{n}\right)\right)$ if, and only if, $A=\operatorname{Op}\left(a_{j}\right)$ for some symbol $\left(a_{j}\right)_{j \in \mathbb{Z}^{n}}$ of order zero: in other words if, and only if, $A$ is a bounded operator on $L^{2}\left(\mathbb{T}^{n}\right)$ defined by

$$
A u(x)=\frac{1}{(2 \pi)^{n}} \sum_{j \in \mathbb{Z}^{n}} a_{j}(x) e_{j}(x) \widehat{u}_{j}, \quad \widehat{u}_{j}:=\int_{\mathbb{T}^{n}} e_{-j} u,
$$

for all $u \in C^{\infty}\left(\mathbb{T}^{n}\right)$ and $x \in \mathbb{T}^{n}$, with $\left(a_{j}\right)_{j \in \mathbb{Z}^{n}}$ satisfying $a_{j} \in C^{\infty}\left(\mathbb{T}^{n}\right), j \in \mathbb{Z}^{n}$, and

$$
\sup \left\{\left|\partial^{\alpha} a_{j}(x)\right| ; j \in \mathbb{Z}^{n}, x \in \mathbb{T}^{n}\right\}<\infty,
$$

for every multi-index $\alpha \in \mathbb{N}^{n}$. For such $A=\mathrm{Op}\left(a_{j}\right)$, one has $T_{y} A T_{-y}=\operatorname{Op}\left(\left(T_{y} a_{j}\right)_{j \in \mathbb{Z}^{n}}\right)$, so $\partial_{y}^{\alpha}\left(T_{y} A T_{-y}\right)=T_{y}\left[\operatorname{Op}\left(\left(\partial^{\alpha} a_{j}\right)_{j \in \mathbb{Z}^{n}}\right)\right] T_{-y}$, for every $\alpha \in \mathbb{N}^{n}$ and $y \in \mathbb{T}^{n}$ - this can be seen by repeated use of the equality

$$
\begin{gathered}
a_{j}\left(x+h f_{k}\right)-a_{j}(x)-h \partial_{k} a_{j}(x)=h \int_{0}^{1}\left[\partial_{k} a_{j}\left(x+t h f_{k}\right)-\partial_{k} a_{j}(x)\right] d t= \\
h^{2} \int_{0}^{1} \int_{0}^{1} \partial_{k}^{2} a_{j}\left(x+t s h f_{k}\right) d s d t
\end{gathered}
$$

where $h \in \mathbb{R}$ and $f_{k}$ denotes the $k^{\text {th }}$ element of the canonical basis of $\mathbb{R}^{n}$, combined with estimates [33, Theorem 1 - (4)]. Therefore, the infinitesimal generators of the (not everywhere strongly continuous) adjoint representation $V: y \longmapsto T_{y}(\cdot) T_{-y}$ restrict to the *-algebra of smooth operators $C^{\infty}(V)$ as operators of the form

$$
\mathrm{Op}\left(a_{j}\right) \longmapsto \mathrm{Op}\left(\sum_{k=1}^{n} c_{k} \partial_{k} a_{j}\right), \quad c_{k} \in \mathbb{R},
$$

and are all generators of $\Gamma_{\infty}$-groups of bounded type (since the range of $\partial V$ is an abelian Lie algebra), with $\Gamma_{\infty}:=\left\{\|\cdot\|_{n}: n \in \mathbb{N}\right\},\|\cdot\|$ being the usual operator norm - this follows from the fact that $y \longmapsto T_{y}(\cdot) T_{-y}$ is a unitary representation. Moreover, their squares are generators of $\Gamma_{\infty}$-semigroups. Also, by [8, Theorem 3.3] and the calculations above, their resolvent operators exist for $\lambda \in \mathbb{C} \backslash i \mathbb{R}$ and belong to $\mathcal{L}_{\Gamma_{\infty}}\left(C^{\infty}(V)\right)$.

\footnotetext{
${ }^{30}$ [33] also characterizes the analytic vectors with respect to the representation $y \longmapsto T_{y}(\cdot) T_{-y}$. A characterization of the smooth vectors in the case of the circle was first obtained in [89], which is a discrete version of the characterization obtained in [41] (see, also, [42, Chapter 8]).
} 
An analogous application can be given for the canonical (not everywhere strongly continuous) action $\alpha$ of the Heisenberg group $H_{2 n+1}(\mathbb{R})$ on the $\mathrm{C}^{*}$-algebra of bounded operators $\mathcal{L}\left(L^{2}\left(\mathbb{R}^{n}\right)\right)$ : Cordes proved in [41] that a bounded linear operator $A$ on $L^{2}\left(\mathbb{R}^{n}\right)$ is such that

$$
\mathbb{R}^{2 n+1} \ni(\mathbf{a}, \mathbf{b}, c) \longmapsto\left(\mathcal{M}_{c} \circ M_{\mathbf{b}} \circ T_{\mathbf{a}}\right) A\left(\mathcal{M}_{c} \circ M_{\mathbf{b}} \circ T_{\mathbf{a}}\right)^{-1} \in \mathcal{L}\left(L^{2}\left(\mathbb{R}^{n}\right)\right)
$$

is a smooth function with values in the $\mathrm{C}^{*}$-algebra of all bounded operators on $L^{2}\left(\mathbb{R}^{n}\right)$ if, and only if, there exists $a \in C^{\infty}\left(\mathbb{R}^{2 n}\right)$, bounded and with all its partial derivatives also bounded - denote this space by $\mathcal{C} \mathcal{B}^{\infty}\left(\mathbb{R}^{2 n}\right)$ - such that, for all $u \in \mathcal{S}\left(\mathbb{R}^{n}\right)$ and all $x \in \mathbb{R}^{n}$, one has

$$
A u(x)=\frac{1}{(2 \pi)^{n}} \int_{\mathbb{R}^{n}} e^{i x \cdot \xi} a(x, \xi) \widehat{u}(\xi) d \xi, \quad \text { with } \quad \widehat{u}(\xi):=\int_{\mathbb{R}^{n}} e^{-i s \cdot \xi} u(s) d s
$$

- in this case, such $A$ is denoted by $a(x, D)$. Therefore, the operators sending these $a(x, D)$ to $\left(\sum_{k=1}^{2 n} c_{k} \partial_{k} a\right)(x, D), c_{k} \in \mathbb{R}$, are generators of $\Gamma_{\infty}$-groups of bounded type, for a natural fundamental system of seminorms $\Gamma_{\infty}$ for $C^{\infty}(\alpha)$ (note, also, that their resolvent operators exist for $\lambda \in \mathbb{C} \backslash i \mathbb{R}$, just as in the previous example), and the squares of these operators generate $\Gamma_{\infty}$-semigroups - to prove that the first $2 n$ canonical directions give rise to generators which act on $a(x, D)$ via a partial differentiation $\partial_{k}, 1 \leq k \leq 2 n$, on the symbol of $a(x, D)$, use the same strategy employed above, in the case of the torus, but with the estimates of the Calderón-Vaillancourt Theorem [34]. Note that the $(2 n+1)^{\text {th }}$ direction gives the zero operator as a generator. There exists an isomorphism of Fréchet spaces between $C^{\infty}(\alpha)$ and $\mathcal{C B}^{\infty}\left(\mathbb{R}^{2 n}\right)$, when the latter is equipped with the topology of uniform convergence of the derivatives. Therefore, as a corollary, the $\lambda$-resolvents of the operators $a \longmapsto \sum_{1 \leq k \leq 2 n} c_{k} \partial_{k} a$ on $\mathcal{C B}^{\infty}\left(\mathbb{R}^{2 n}\right)$ exist for $\lambda \in \mathbb{C} \backslash i \mathbb{R}$, so that they are continuous bijective linear operators on $\mathcal{C B}^{\infty}\left(\mathbb{R}^{2 n}\right)$.

Following [42], denote $C^{\infty}(\alpha)$ by $\Psi G T$ and equip the set

$$
g l:=\left\{(g, \mathbf{a}, \mathbf{b}, c): g \in G L\left(\mathbb{R}^{n}\right), \mathbf{a}, \mathbf{b} \in \mathbb{R}^{n}, c \in \mathbb{R} /(2 \pi \mathbb{Z})\right\}
$$

with a Lie group structure, as in [42, page 265]. If $\mathcal{T}$ is the unitary representation of $g l$ defined by

$$
\left(\mathcal{T}_{g, \mathbf{a}, \mathbf{b}, c} u\right)(x):=|\operatorname{det} g|^{1 / 2} e^{i c} e^{i\langle\mathbf{b}, x\rangle} u(g x+\mathbf{a}),
$$

then the smooth vectors for the adjoint representation, denoted by $\Psi G L$, consists precisely of the elements $a(x, D)$ in $\Psi G T$ such that their symbols $a \in \mathcal{C B}^{\infty}\left(\mathbb{R}^{2 n}\right)$ remain in $\mathcal{C B}^{\infty}\left(\mathbb{R}^{2 n}\right)$ after any finite number of applications of the operators

$$
\epsilon_{j l}:=\xi_{j} \partial_{\xi_{l}}-x_{l} \partial_{x_{j}}, \quad j, l=1, \ldots n,
$$

by [42, Theorem 5.3, page 269]. Moreover, by [42, Equation (5.24), page 268], the operators

$$
a(x, D) \longmapsto\left(\sum_{1 \leq k \leq 2 n} c_{k} \partial_{k} a+\sum_{1 \leq j, l \leq n} d_{j l} \epsilon_{j l} a\right)(x, D), \quad c_{k}, d_{j l} \in \mathbb{R},
$$


are generators of $\Gamma_{\infty}$-groups, and their squares generate $\Gamma_{\infty}$-semigroups. A similar result may be obtained if one substitutes $g l$ by the subgroup

$$
g s:=\left\{(\sigma Q, \mathbf{a}, \mathbf{b}, c): \sigma>0, Q \in S O(n), \mathbf{a}, \mathbf{b} \in \mathbb{R}^{n}, c \in \mathbb{R} /(2 \pi \mathbb{Z})\right\}
$$

and considers the subsequent space of smooth vectors $\Psi G S$ - see [42, page 265] and [42, Theorem 5.4, page 269]. Note that $\Psi G L \subseteq \Psi G S \subseteq \Psi G T{ }^{31}$

\subsection{Some Estimates Involving Lie Algebras}

Let $(\mathcal{X}, \Gamma)$ be a locally convex space, $\mathcal{D} \subseteq \mathcal{X}$ and $\mathcal{L} \subseteq \operatorname{End}(\mathcal{D})$ be a real finitedimensional Lie algebra of linear operators acting on $\mathcal{X}$, with an ordered basis $\left(B_{k}\right)_{1 \leq k \leq d}$. Then, for each $1 \leq i, j \leq d$, one has

$$
(*) \quad\left(\operatorname{ad} B_{i}\right)\left(B_{j}\right):=\left[B_{i}, B_{j}\right]=\sum_{k=1}^{d} c_{i j}^{(k)} B_{k},
$$

for some constants $c_{i j}^{(k)} \in \mathbb{R}$. Therefore, if $B^{\mathrm{u}}$ and $B^{\vee}$ are two monomials in the variables $\left(B_{k}\right)_{1 \leq k \leq d}$, applying recursively the identity above it is possible to prove that the element $\left(\operatorname{ad} B^{\mathrm{u}}\right)\left(B^{\mathrm{v}}\right):=B^{\mathrm{u}} B^{\mathrm{v}}-B^{\mathrm{v}} B^{\mathrm{u}}$, which has size $|\mathrm{u}|+|\mathrm{v}|$, is actually a sum of terms of size at most $|\mathrm{u}|+|\mathrm{v}|-1$. To see this, one must proceed recursively: if $B^{\mathrm{u}}:=B_{\mathrm{u}_{1}} \ldots B_{\mathrm{u}_{|\mathrm{u}|}}$ and $B^{\mathrm{v}}:=B_{\mathrm{v}_{1}} \ldots B_{\mathrm{v}_{|\mathrm{v}|}}$, then switching the last element of $B^{\mathrm{u}}$ with the first element of $B^{\mathrm{v}}$, and using relation $(*)$, gives

$$
\begin{gathered}
B^{\mathrm{u}} B^{\mathrm{v}}=B_{\mathrm{u}_{1}} \ldots B_{\mathrm{v}_{1}} B_{\mathrm{u}_{|\mathrm{u}|}} \ldots B_{\mathrm{v}_{|\mathrm{v}|}}+B_{\mathrm{u}_{1}} \ldots \text { ad }\left(B_{\mathrm{u}_{\mid \mathrm{u}} \mid}\right)\left(B_{\mathrm{v}_{1}}\right) \ldots B_{\mathrm{v}_{|\mathrm{v}|}} \\
\left.=B_{\mathrm{u}_{1}} \ldots B_{\mathrm{v}_{1}} B_{\mathrm{u}_{|\mathrm{u}|}} \ldots B_{\mathrm{v}_{|\mathrm{v}|}}+\text { (linear combination of } d \text { terms of size at most }|\mathrm{u}|+|\mathrm{v}|-1\right) .
\end{gathered}
$$

Therefore, by iterating this process one concludes that, after repeating this step $|u| \cdot|v|$ times, the identity

$$
(* *) \quad\left(\operatorname{ad} B^{\mathrm{u}}\right)\left(B^{\mathrm{v}}\right):=B^{\mathrm{u}} B^{\mathrm{v}}-B^{\mathrm{v}} B^{\mathrm{u}}=\sum_{|\mathrm{w}| \leq|\mathrm{u}|+|\mathrm{v}|-1} c_{\mathrm{w}} B^{\mathrm{w}}
$$

arises, where the sum has, at most, $d \cdot|\mathrm{u}| \cdot|\mathrm{v}|$ summands. With all of this in mind, one can obtain the estimates

$$
p\left(\left(\operatorname{ad} B^{\mathrm{u}}\right)\left(B^{\mathrm{v}}\right)(x)\right) \leq k|\mathrm{u}||\mathbf{v}| \rho_{p,|\mathrm{u}|+|\mathrm{v}|-1}(x), \quad x \in \mathcal{D}, p \in \Gamma,
$$

\footnotetext{
${ }^{31}$ The author would like to thank professor Severino T. Melo for suggesting the study of the examples in this last paragraph.
} 
where $k$ is a non-negative constant which does not depend on $\mathrm{u}$ or $\mathrm{v}$, and is related only with the coefficients that come from $(*)$ - remind that the seminorm $\rho_{p,|\mathbf{u}|+|\mathbf{v}|-1}$ was introduced in 1.2.4.

These estimates will be extremely useful in Chapter 2. ${ }^{32}$ Also, Lemma 1.5.1, below, will be an important tool for the proofs of Theorems 2.11 and 2.12. A product rule-type theorem for locally convex spaces will be necessary for its proof, so its statement will be written for the sake of completeness:

The Product Rule, [74, Theorem A.1, page 440]: Let E, F be Hausdorff locally convex spaces and $I \subseteq \mathbb{R}$ an open interval. Let $K: I \longrightarrow \mathcal{L}_{s}(E, F)$ be a differentiable locally equicontinuous mapping (with $\mathcal{L}_{s}(E, F)$ being the space of all continuous linear maps from $E$ to $F$ equipped with the strong operator topology) and let $f: I \longrightarrow E$ be differentiable. Then, the product mapping $H: I \longrightarrow F$ defined by $H(t):=K(t)(f(t))$, for $t \in I$, is differentiable. The first-order derivative is given by

$$
H^{\prime}(t)=K(t) f^{\prime}(t)+K^{\prime}(t) f(t), \quad t \in I .
$$

Lemma 1.5.1: Let $B^{\mathrm{u}}$ and $B^{\vee}$ be two monomials in the basis elements $\left(B_{k}\right)_{1 \leq k \leq d}$. Suppose $H_{m}$ is an element of order $m$ in the complexification $\mathfrak{U}(\mathcal{L})_{\mathbb{C}}$ of the universal enveloping algebra of $\mathcal{L}$ such that $-H_{m}$ is a pregenerator of a strongly continuous locally equicontinuous semigroup $t \longmapsto S_{t}$ satisfying $S_{t}[\mathcal{X}] \subseteq \mathcal{D}$, for all $t>0$, and

$$
\rho_{p, n}(x) \leq \epsilon^{m-n} p\left(H_{m}(x)\right)+\frac{N_{p}}{\epsilon^{n}} p(x), \quad p \in \Gamma, x \in \mathcal{D},
$$

for all $0<n \leq m-1,0<\epsilon \leq 1$ and a constant $N_{p}>0$ (alternatively, the symbol $S(t)$ will sometimes be used to denote the operator $\left.S_{t}\right)$. Define the element $\left(\right.$ ad $\left.B^{\mathrm{u}}\right)\left(H_{m}\right)$ of $\mathfrak{U}(\mathcal{L})_{\mathbb{C}}$ via an extension of the operator ad $B^{\mathrm{u}}$ just defined in $(* *)$, by linearity. Then, the identity ${ }^{33}$

$$
\int_{0}^{s} B^{\vee} S_{r}\left[\left(a d B^{\mathrm{u}}\right)\left(H_{m}\right)\right] S_{t-r}(x) d r=B^{\vee}\left[\left(a d S_{s}\right)\left(B^{\mathrm{u}}\right)\right] S_{t-s}(x), \quad x \in \mathcal{D}, 0 \leq s \leq t
$$

is valid, for all $t>0$, where $\left(a d S_{s}\right)\left(B^{\mathrm{u}}\right)$ is defined to be the operator $S_{s} B^{\mathrm{u}}-B^{\mathrm{u}} S_{s}$ on $\mathcal{D}$.

Proof of Lemma 1.5.1: Fix $t>0$. The first task will be to establish continuity of the function $r \longmapsto B^{\vee} S_{r} B^{\mathrm{u}} S_{t-r}(x)$ on $[0, t]$, for all $x \in \mathcal{D}$. To this purpose, it will first be obtained the differentiability of $r \longmapsto B^{\mathrm{w}} S_{r}(x)$ at $r_{0} \in[0, t]$, for all monomials $B^{\mathrm{w}}$ of size

\footnotetext{
${ }^{32}$ See also [109, page 78$]$.

${ }^{33}$ In the case where $H_{m}=-\sum_{k=1}^{d} B_{k}^{2},[25$, page 356] refers to this identity as Duhamel formula.
} 
$(q-1)(m-1) \leq|\mathrm{w}| \leq q(m-1)$ in the elements of $\left(B_{k}\right)_{1 \leq k \leq d}$ and $x \in \mathcal{D}$, a fact which will be proved by induction on $q \geq 1$. To deal with the case $q=1$, first note that

$$
\frac{S_{r}-S_{r_{0}}}{r-r_{0}} x=S_{r_{0}} \frac{S_{r-r_{0}}-I}{r-r_{0}} x, \quad \text { if } r>r_{0}
$$

and

$$
\frac{S_{r}-S_{r_{0}}}{r-r_{0}} x=S_{r} \frac{I-S_{r_{0}-r}}{r-r_{0}} x, \quad \text { if } r<r_{0},
$$

so the fact that $H_{m} S_{s}=S_{s} H_{m}$ on $\mathcal{D}$, for all $s \in[0,+\infty)$, together with (1.5.1.1), gives

$$
\begin{gathered}
p\left(B^{\mathrm{w}} S_{r_{0}}\left(\frac{S_{r-r_{0}}-I}{r-r_{0}} x+H_{m}(x)\right)\right) \leq \epsilon^{m-n} p\left(S_{r_{0}}\left(\frac{S_{r-r_{0}}-I}{r-r_{0}} H_{m}(x)+H_{m}^{2}(x)\right)\right) \\
+\frac{N_{p}}{\epsilon^{n}} p\left(S_{r_{0}}\left(\frac{S_{r-r_{0}}-I}{r-r_{0}} x+H_{m}(x)\right)\right), \quad x \in \mathcal{D}, p \in \Gamma,
\end{gathered}
$$

if $r>r_{0}$, and

$$
\begin{gathered}
p\left(B^{\mathrm{w}} S_{r}\left(\frac{I-S_{r_{0}-r}}{r-r_{0}} x+H_{m}(x)\right)\right) \leq \epsilon^{m-n} p\left(S_{r}\left(\frac{I-S_{r_{0}-r}}{r-r_{0}} H_{m}(x)+H_{m}^{2}(x)\right)\right) \\
\quad+\frac{N_{p}}{\epsilon^{n}} p\left(S_{r}\left(\frac{I-S_{r_{0}-r}}{r-r_{0}} x+H_{m}(x)\right)\right), \quad x \in \mathcal{D}, p \in \Gamma,
\end{gathered}
$$

if $r<r_{0}$, for all $0<\epsilon \leq 1$. Also,

$$
\begin{gathered}
\frac{S_{r}-S_{r_{0}}}{r-r_{0}} x+S_{r_{0}} H_{m}(x)=S_{r}\left(\frac{I-S_{r_{0}-r}}{r-r_{0}} x+H_{m}(x)\right) \\
+\left(S_{r_{0}}-S_{r}\right) H_{m}(x), \quad r<r_{0} .
\end{gathered}
$$

Joining all these informations together yields the desired differentiability.

Now, suppose $r \longmapsto B^{\mathrm{w}} S_{r}(x)$ is differentiable at $r_{0} \in[0, t]$, for all $x \in \mathcal{D}$ and all monomials $B^{\mathrm{w}}$ in the elements of $\left(B_{k}\right)_{1 \leq k \leq d}$, with $(q-1)(m-1) \leq|\mathrm{w}| \leq q(m-1)$, for some $q \geq 1$. A fixed monomial $B^{\mathrm{w}}$ of size $q(m-1) \leq|\mathrm{w}|:=n \leq(q+1)(m-1)$ may be decomposed as $B^{\mathrm{w}}=B^{\mathrm{w}_{0}} B^{\mathrm{w}^{\prime}}$, with $\left|\mathrm{w}_{0}\right|=m-1$ and $\left|\mathrm{w}^{\prime}\right|=n-(m-1)$. Using (1.5.1.1), one obtains

$$
\begin{gathered}
p\left(B^{\mathrm{w}} S(r) x\right)=p\left(B^{\mathrm{w}_{0}} B^{\mathrm{w}^{\prime}} S(r) x\right) \\
\leq \epsilon p\left(H_{m} B^{\mathrm{w}^{\prime}} S(r) x\right)+\frac{N_{p}}{\epsilon^{m-1}} p\left(B^{\mathrm{w}^{\prime}} S(r) x\right), \quad p \in \Gamma, x \in \mathcal{D},
\end{gathered}
$$

for all $r \in[0,+\infty)$ and $0<\epsilon \leq 1$. On the other hand,

$$
p\left(H_{m} B^{\mathrm{w}^{\prime}} S(r) x\right) \leq p\left(\left[\operatorname{ad}\left(H_{m}\right)\left(B^{\mathrm{w}^{\prime}}\right)\right] S(r) x\right)+p\left(B^{\mathrm{w}^{\prime}} H_{m} S(r) x\right)
$$




$$
\leq k(n-(m-1)) m \rho_{p, n}(S(r) x)+p\left(B^{\mathbf{w}^{\prime}} H_{m} S(r) x\right),
$$

$k$ being a constant depending only on $d, H_{m}$ and on the numbers $c_{i j}^{(k)}$ defined by $\left[B_{i}, B_{j}\right]=$ $\sum_{k=1}^{d} c_{i j}^{(k)} B_{k}$. Hence,

$$
\begin{aligned}
p\left(B^{\mathrm{w}} S(r) x\right) \leq & \epsilon\left[k(n-(m-1)) m \rho_{p, n}(S(r) x)+p\left(B^{\mathrm{w}^{\prime}} H_{m} S(r) x\right)\right] \\
& +\frac{N_{p}}{\epsilon^{m-1}} p\left(B^{\mathrm{w}^{\prime}} S(r) x\right), \quad p \in \Gamma, x \in \mathcal{D},
\end{aligned}
$$

for all $r \in[0,+\infty)$ and $0<\epsilon \leq 1$. Choosing an $1 \geq \epsilon_{0}>0$ such that $\epsilon_{0} k(n-(m-1)) m<1$, and taking the maximum over all monomials $B^{\mathrm{w}}$ of size $n$, gives

$$
p\left(B^{\mathrm{w}} S(r) x\right) \leq \rho_{p, n}(S(r) x) \leq \frac{\epsilon_{0} \rho_{p, n-(m-1)}\left(H_{m} S(r) x\right)+\frac{N_{p}}{\epsilon^{m-1}} \rho_{p, n-(m-1)}(S(r) x)}{1-\epsilon_{0} k(n-(m-1))},
$$

so the induction hypothesis together with a similar argument made in the case $q=1$ ends the induction proof.

Fix $t>0, x \in \mathcal{D}, g \in \mathcal{X}^{\prime}$ and define on $[0, t]$ the functions

$$
g_{1}: s \longmapsto \int_{0}^{s} g\left(B^{\vee} S_{r}\left[\left(\operatorname{ad} B^{\mathrm{u}}\right)\left(H_{m}\right)\right] S_{t-r}(x)\right) d r
$$

and

$$
g_{2}: s \longmapsto g\left(B^{\mathrm{v}}\left[\left(\operatorname{ad} S_{s}\right)\left(B^{\mathrm{u}}\right)\right] S_{t-s}(x)\right)=g\left(B^{\mathrm{v}} S_{s} B^{\mathrm{u}} S_{t-s}(x)\right)-g\left(B^{\mathrm{v}} B^{\mathrm{u}} S_{t}(x)\right) .
$$

An application of [74, Theorem A.1, page 440] together with what was just proved gives, in particular, that $r \longmapsto g\left(B^{\vee} S_{r}\left(\operatorname{ad} B^{\mathrm{u}}\right)\left(H_{m}\right) S_{t-r}(x)\right)$ is continuous on $[0, t]$, so the integral

$$
\int_{0}^{s} g\left(B^{\vee} S_{r}\left[\left(\operatorname{ad} B^{\mathrm{u}}\right)\left(H_{m}\right)\right] S_{t-r}(x)\right) d r
$$

defines a differentiable function on $[0, t]$ with $g_{1}^{\prime}(s)=g\left(B^{\vee} S_{s}\left[\left(\operatorname{ad} B^{\mathrm{u}}\right)\left(H_{m}\right)\right] S_{t-s}(x)\right)$. If $\mathcal{D}$ is equipped with the $\mathrm{C}^{\infty}$ projective topology, then it is clear by the induction proof above that $f: s \longmapsto B^{\mathrm{u}} S_{t-s}(x)$ is a differentiable map from $[0, t]$ to $\mathcal{D}$ and $f^{\prime}(s)=B^{\mathrm{u}} S_{t-s} H_{m}(x)$. The same applies for the function $s \longmapsto B^{\vee} S_{s}(y)$, for every fixed $y \in \mathcal{D}$. Therefore, by the product rule in [74, Theorem A.1, page 440], one sees that $H: s \longmapsto B^{\vee} S_{s} B^{\mathrm{u}} S_{t-s}(x)$ is differentiable on $(0, t)$ and, defining $K: s \longmapsto B^{\vee} S_{s}$, it follows that

$$
\begin{gathered}
H^{\prime}(s)=[K(s)(f(s))]^{\prime}=K^{\prime}(s) f(s)+K(s) f^{\prime}(s) \\
=-B^{\vee} S_{s} H_{m} B^{\mathrm{u}} S_{t-s}(x)+B^{\vee} S_{s} B^{\mathrm{u}} S_{t-s} H_{m}(x)=B^{\vee} S_{s}\left[\left(\operatorname{ad} B^{\mathrm{u}}\right)\left(H_{m}\right)\right] S_{t-s}(x),
\end{gathered}
$$


where the derivative $K^{\prime}$ is taken with respect to the strong operator topology. This implies $g_{1}^{\prime}=g_{2}^{\prime}$ on $(0, t)$ and, since $g_{1}(0)=g_{2}(0)=0, g_{1}$ must be equal to $g_{2}$ on $[0, t]$. By a corollary of the Hahn-Banach Theorem, the equality

$$
\int_{0}^{s} B^{\vee} S_{r}\left[\left(\operatorname{ad} B^{\mathrm{u}}\right)\left(H_{m}\right)\right] S_{t-r}(x) d r=B^{\mathrm{v}}\left[\left(\operatorname{ad} S_{s}\right)\left(B^{\mathrm{u}}\right)\right] S_{t-s}(x), \quad x \in \mathcal{D}, 0 \leq s \leq t,
$$

must hold.

\subsection{Extending Continuous Linear Maps}

In many situations of the thesis it will be of interest to extend continuous linear operators to the closure of the original domain. To this purpose, the following theorem is going to be repeatedly used:

Lemma 1.6.1: Let $\left(X, \Gamma_{\mathcal{X}}\right)$ and $\left(Y, \Gamma_{\mathcal{Y}}\right)$ be locally convex spaces, $\mathcal{Y}$ being Hausdorff and complete. If $\mathcal{D} \subseteq \mathcal{X}$ is a linear subspace of $\mathcal{X}$ and $T: \mathcal{D} \longrightarrow \mathcal{Y}$ is a continuous linear map then there exists a unique linear map $\tilde{T}: \overline{\mathcal{D}} \longrightarrow \mathcal{Y}$ such that $\left.\tilde{T}\right|_{\mathcal{D}}=T$. In particular, if $\mathcal{D}$ is dense in $\mathcal{X}$ then there exists a unique continuous linear map $\tilde{T}: \mathcal{X} \longrightarrow \mathcal{Y}$ such that $\left.\tilde{T}\right|_{\mathcal{D}}=T$.

Proof of Lemma 1.6.1: Let $x \in \overline{\mathcal{D}}$ and $\left\{x_{\alpha}\right\}_{\alpha \in \mathcal{A}}$ be a net in $\mathcal{D}$ such that $x_{\alpha} \longrightarrow x$. Then, it will be shown that the map $\tilde{T}$ defined by $\tilde{T} x:=\lim _{\alpha} T x_{\alpha}$ is well-defined, linear and continuous. First, note that for each fixed $p \in \Gamma_{\mathcal{Y}}$ there exist $C_{p}>0$ and $q \in \Gamma_{\mathcal{X}}$ satisfying

$$
p\left(T x_{\alpha_{1}}-T x_{\alpha_{2}}\right)=p\left(T\left(x_{\alpha_{1}}-x_{\alpha_{2}}\right)\right) \leq C_{p} q\left(x_{\alpha_{1}}-x_{\alpha_{2}}\right),
$$

for all $\alpha_{1}, \alpha_{2} \in \mathcal{A}$, showing that $\left\{T x_{\alpha}\right\}_{\alpha \in \mathcal{A}}$ is a Cauchy net in $\mathcal{Y}$, so the completeness of $\mathcal{Y}$ implies the existence of the $\operatorname{limit} \lim T x_{\alpha}$ in $\mathcal{Y}$. Besides, this limit is unique, due to the fact that $\mathcal{Y}$ is Hausdorff. Now, if $\left\{y_{\beta}\right\}_{\beta \in \mathcal{B}}$ is a net in $\mathcal{D}$ such that $y_{\beta} \longrightarrow x$, then the calculation

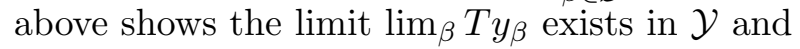

$$
p\left(T x_{\alpha}-T y_{\beta}\right)=p\left(T\left(x_{\alpha}-y_{\beta}\right)\right) \leq C_{p} q\left(x_{\alpha}-y_{\beta}\right),
$$

for every $\alpha \in \mathcal{A}$ and $\beta \in \mathcal{B}$. This shows

$$
\lim _{\alpha} \lim _{\beta}\left(T x_{\alpha}-T y_{\beta}\right)=\lim _{\beta} \lim _{\alpha}\left(T x_{\alpha}-T y_{\beta}\right)=0
$$

so

$$
\lim _{\beta} T y_{\beta}=\lim _{\alpha} \lim _{\beta} T y_{\beta}=\lim _{\alpha} \lim _{\beta}\left(T y_{\beta}-T x_{\alpha}\right)+\lim _{\alpha} \lim _{\beta} T x_{\alpha}=\lim _{\alpha} \lim _{\beta} T x_{\alpha}=\lim _{\alpha} T x_{\alpha} .
$$


This establishes that $\tilde{T}$ is well-defined, since it is independent of the particular choice of the net converging to $x$. Linearity of $\tilde{T}$ follows from the fact that if $z \in \overline{\mathcal{D}}$, with $\left\{z_{\gamma}\right\}_{\gamma \in \mathcal{C}} \subseteq \mathcal{D}$ and $z_{\gamma} \longrightarrow z$, then

$$
\begin{gathered}
\tilde{T}(\mu x+z):=\lim _{\alpha} \lim _{\gamma} T\left(\mu x_{\alpha}+z_{\gamma}\right)=\lim _{\alpha} \lim _{\gamma}\left(\mu T x_{\alpha}+T z_{\gamma}\right) \\
=\mu \lim _{\alpha} \lim _{\gamma} T x_{\alpha}+\lim _{\alpha} \lim _{\gamma} T z_{\gamma}=\mu \tilde{T} x+\tilde{T} z .
\end{gathered}
$$

Finally, $\tilde{T}$ is continuous, for if $p$ belongs to $\Gamma_{\mathcal{Y}}$, there exist $C_{p}>0$ and $q \in \Gamma_{\mathcal{X}}$ such that

$$
p\left(T x_{\alpha}\right) \leq C_{p} q\left(x_{\alpha}\right)
$$

for all $\alpha \in \mathcal{A}$ so, taking limits in $\alpha$ on both members of the inequality yields

$$
p(\tilde{T} x) \leq C_{p} q(x) .
$$

Therefore, since $p \in \Gamma_{\mathcal{Y}}$ and $x \in \mathcal{X}$ are arbitrary, $\tilde{T}$ is continuous in $\overline{\mathcal{D}}$. To see $\left.\tilde{T}\right|_{\mathcal{D}}=T$, fix $x \in \mathcal{D}$ and the constant net $\left\{x_{\alpha}\right\}_{\alpha \in \mathcal{A}}, x_{\alpha}:=x$, for all $\alpha \in \mathcal{A}$. Then, $\tilde{T} x=\lim _{\alpha} T x_{\alpha}=$ $\lim _{\alpha} T x=T x$.

To prove uniqueness of such extension, suppose there exists a map $G: \overline{\mathcal{D}} \longrightarrow \mathcal{Y}$ with $\left.G\right|_{D}=T$. If $x \in \bar{D}$ and $\left\{x_{\alpha}\right\}_{\alpha \in \mathcal{A}}$ is a net in $\mathcal{D}$ converging to $x$, then from the continuity of $G$ it follows that

$$
G x=G\left(\lim _{\alpha} x_{\alpha}\right)=\lim _{\alpha} G x_{\alpha}=\lim _{\alpha} T x_{\alpha}=: \tilde{T} x
$$

so the assertion follows.

\subsection{Projective Limits}

Definition (Projective Limits): Let $\left\{\mathcal{X}_{i}\right\}_{i \in I}$ be a family of Hausdorff locally convex spaces, where $I$ is a directed set under the partial order $\preceq$ and, for all $i, j \in I$ satisfying $i \preceq j$, suppose that there exists a continuous linear map $\mu_{i j}: \mathcal{X}_{j} \longrightarrow \mathcal{X}_{i}$ satisfying $\mu_{i j} \circ \mu_{j k}=$ $\mu_{i k}$, whenever $i \preceq j \preceq k$ ( $\mu_{i i} i s$, by definition, the identity map, for all $\left.i \in I\right)$. Then, $\left(\mathcal{X}_{i}, \mu_{i j}, I\right)$ is called a projective system (or an inverse system) of Hausdorff locally convex spaces. Consider the vector space $\mathcal{X}$ defined as

$$
\mathcal{X}:=\left\{\left(x_{i}\right)_{i \in I} \in \prod_{i \in I} \mathcal{X}_{i}: \mu_{i j}\left(x_{j}\right)=x_{i}, \text { for all } i, j \in I \text { with } i \preceq j\right\}
$$

and equipped with the relative Tychonoff's product topology or, equivalently, the coarsest topology for which every canonical projection $\pi_{j}:\left(x_{i}\right)_{i \in I} \longmapsto x_{j}$ is continuous, relativized to $\mathcal{X}$. Then, $\mathcal{X}$ is a Hausdorff locally convex space, and is called the projective limit (or 
inverse limit) of the family $\left\{\mathcal{X}_{i}\right\}_{i \in I}$. In this case, the notation $\lim \mathcal{X}_{i}:=\mathcal{X}$ is employed. If $\pi_{i}[\mathcal{X}]$ is dense in each $\mathcal{X}_{i}$, then the projective limit is said to be reduced. There is no loss of generality in assuming a projective limit to be reduced: indeed, the projective limit of the family

$$
\left\{\overline{\pi_{i}[\mathcal{X}]}\right\}_{i \in I}
$$

is equal to the projective limit of $\left\{\mathcal{X}_{i}\right\}_{i \in I}$, which is $\mathcal{X}$ (see [117, page 139]).

If $(\mathcal{Y}, \Gamma)$ is a complete Hausdorff locally convex space, then it is isomorphic to a particular projective limit:

Lemma 1.7.1: A complete Hausdorff locally convex space $(\mathcal{Y}, \Gamma)$ is isomorphic to the projective limit $\lim \mathcal{Y}_{p}$, where $\mathcal{Y}_{p}$ is the Banach completion of $\mathcal{Y} / N_{p}$, for every $p \in \Gamma-$ by an isomorphism between two locally convex spaces is meant a continuous bijective linear map with a continuous inverse.

Proof of Lemma 1.7.1: Since $\Gamma$ is saturated, it is a directed set. Moreover, if $p, q \in \Gamma$ satisfy $p \preceq q$, define the continuous linear map $\mu_{p q}: \mathcal{Y}_{q} \longrightarrow \mathcal{Y}_{p}$ as the unique bounded linear extension of the map $[x]_{q} \longmapsto[x]_{p}$. Then, the relations $\mu_{p r}=\mu_{p q} \circ \mu_{q r}$ hold, whenever $p, q, r \in \Gamma$ satisfy $p \preceq q \preceq r$, so the projective limit $\lim \mathcal{Y}_{p}$ is well-defined. The map

$$
\Phi: \mathcal{Y} \longrightarrow \prod_{p \in \Gamma} \mathcal{Y}_{p}, \quad \Phi(x):=\left([x]_{p}\right)_{p \in \Gamma}
$$

is linear and injective, because $\mathcal{Y}$ is Hausdorff. Also, by the definition of $\Phi$, it is clear that $\Phi[\mathcal{Y}] \subseteq \lim _{\longleftarrow} \mathcal{Y}_{p}$. To show $\Phi[\mathcal{Y}]=\lim _{\longleftarrow} \mathcal{Y}_{p}$, two auxiliary steps are needed:

- $\Phi[\mathcal{Y}]$ is a closed subspace of $\lim _{\longleftarrow} \mathcal{Y}_{p}:$ consider $x=\left(x_{p}\right)_{p \in \Gamma} \in \overline{\Phi[\mathcal{Y}]} \subseteq \lim _{\longleftarrow} \mathcal{Y}_{p}$ and a net

$$
\left\{\tilde{x}_{\alpha}=\left(\left[x_{\alpha}\right]_{p}\right)_{p \in \Gamma}\right\}_{\alpha \in \mathcal{A}}
$$

in $\Phi[\mathcal{Y}]$ which converges to $x$. For each fixed $p \in \Gamma,\left\{\left[x_{\alpha}\right]_{p}\right\}_{\alpha \in \mathcal{A}}$ is a Cauchy net in $\mathcal{Y} / N_{p}$, which is equivalent to the fact that $\left\{x_{\alpha}\right\}_{\alpha \in \mathcal{A}}$ is a Cauchy net in $\mathcal{Y}$. Since $\mathcal{Y}$ is complete, there exists $y \in \mathcal{Y}$ for which $\lim _{\alpha} x_{\alpha}=y$. This implies $\lim _{\alpha}\left[x_{\alpha}\right]_{p}=[y]_{p}$, for every $p \in \Gamma$, so $x=\left([y]_{p}\right)_{p \in \Gamma} \in \Phi[\mathcal{Y}]$. This ends the proof of this first step.

- $\Phi[\mathcal{Y}]$ is a dense subspace of $\lim _{\longleftarrow} \mathcal{Y}_{p}$ : for every fixed $p \in \Gamma$ the inclusions

$$
\mathcal{Y} / N_{p}=\pi_{p}[\Phi[\mathcal{Y}]] \subseteq \pi_{p}\left[\lim _{\longleftarrow} \mathcal{Y}_{p}\right] \subseteq \mathcal{Y}_{p}
$$

hold, so the fact that $\mathcal{Y} / N_{p}$ is dense in $\mathcal{Y}_{p}$ together with the definition of Tychonoff topology gives the desired result. 
This establishes the equality $\Phi[\mathcal{Y}]=\lim _{\longleftarrow} \mathcal{Y}_{p}$, and a small adaptation of the argument presented in the first item also shows that $\Phi^{-1}$ is an isomorphism. This ends the proof.

Analogous results hold for algebras and $*$-algebras: see [53, pages 16, 32], [90, Theorem $5.1]$.

Now, suppose there are linear operators $T_{i}$ : Dom $T_{i} \subseteq \mathcal{X}_{i} \longrightarrow \mathcal{X}_{i}$ which are connected by the relations $\mu_{i j}\left[\operatorname{Dom} T_{j}\right] \subseteq \operatorname{Dom} T_{i}$ and $T_{i} \circ \mu_{i j}=\mu_{i j} \circ T_{j}$, whenever $i \preceq j$. Then, the family $\left\{T_{i}\right\}_{i \in I}$ is said to be a projective family of linear operators. The latter relation ensures that the linear transformation $T$ defined on Dom $T:=\lim \operatorname{Dom} T_{i} \subseteq \lim \mathcal{X}_{i}$ by $T\left(x_{i}\right)_{i \in I}:=\left(T_{i}\left(x_{i}\right)\right)_{i \in I}$ has its range inside $\varliminf_{\leftarrow} \mathcal{X}_{i}$, thus defining a linear operator on $\varliminf_{\longleftarrow} \mathcal{X}_{i}$. This operator is called the projective limit of $\left\{\boldsymbol{T}_{\boldsymbol{i}}\right\}_{\boldsymbol{i} \in \boldsymbol{I}}$, as in [8, page 167].

With these definitions in mind, the next objective is to introduce projective limits of group representations, so let $G$ be a Lie group and $V_{i}: G \longrightarrow \mathcal{X}_{i}$ be (not necessarily strongly continuous) representations of $G$ satisfying $V_{i}(g) \circ \mu_{i j}=\mu_{i j} \circ V_{j}(g)$, whenever $i \preceq j$. Defining for each fixed $g \in G$ the operator $V(g)$ as the projective limit of $\left\{V_{i}(g): i \in I\right\}$ yields a representation of $G$ on $\lim _{\leftarrow} \mathcal{X}_{i}$, which is called the projective limit of $\left\{V_{i}: i \in I\right\}$. Regarding this representation, the following results are true (they were taken from [91, Lemma 7, page 26]):

1. if every $V_{i}$ is locally equicontinuous, then so is $V$;

2. if every $V_{i}$ is strongly continuous, then so is $V$.

Proof of 1: Fix a compact set $K \subseteq G$, let $W:=\pi_{i}^{-1}\left[W_{i}\right]$ be a basic neighborhood of $0 \in \lim _{\longleftarrow} \mathcal{X}_{i}$, where $W_{i}$ is a basic neighborhood of the origin of $\mathcal{X}_{i}$, and fix $g \in G$. The first step will be to prove that $\cap_{g \in K}(T(g))^{-1}[W]$ is a neighborhood of the origin.

If $x \in(T(g))^{-1}[W]$, then there exists $y \in W_{i}$ such that $y=\pi_{i}(T(g) x)=T_{i}(g)\left(\pi_{i}(x)\right)$, so it is clear that $(T(g))^{-1}[W] \subseteq \pi_{i}^{-1}\left[T_{i}(g)^{-1}\left[W_{i}\right]\right]$. On the other hand, $x \in \pi_{i}^{-1}\left[T_{i}(g)^{-1}\left[W_{i}\right]\right]$ implies $\pi_{i}(T(g) x)=T_{i}(g)\left(\pi_{i}(x)\right) \in W_{i}$, so the other inclusion also holds. Therefore,

$$
\bigcap_{g \in K}(T(g))^{-1}[W]=\bigcap_{g \in K} \pi_{i}^{-1}\left[T_{i}(g)^{-1}\left[W_{i}\right]\right] \supseteq \pi_{i}^{-1}\left[\bigcap_{g \in K} T_{i}(g)^{-1}\left[W_{i}\right]\right] .
$$

Local equicontinuity of each $V_{i}$ guarantees the existence of a neighborhood $U_{i}$ of the origin of $\mathcal{X}_{i}$ such that

$$
U_{i} \subseteq \bigcap_{g \in K} T_{i}(g)^{-1}\left[W_{i}\right], \quad i \in I,
$$


so $\cap_{g \in K}(T(g))^{-1}[W]$ must be a neighborhood of the origin of $\lim _{\longleftarrow} \mathcal{X}_{i}$.

Hence,

$$
T(g)\left[\cap_{g \in K}(T(g))^{-1}[W]\right] \subseteq W,
$$

for all $g \in K$, which establishes the local equicontinuity claim.

Proof of 2: Fix $x=\left(x_{i}\right)_{i \in I} \in \lim \mathcal{X}_{i}$. Since each $V_{i}$ is strongly continuous, the function $g \longmapsto \pi_{i}(V(g) x)=V_{i}(g)\left(\pi_{i}(x)\right)=V_{i}(g)\left(x_{i}\right)$ of $G$ into $\mathcal{X}_{i}$ is continuous, for every $i \in I$. Therefore, by the definition of Tychonoff topology, $g \longmapsto V(g) x$ is a continuous function, so $V$ is strongly continuous.

Some examples of projective limits are: ${ }^{34}$

1. [53, Example (2), page 19]: The space of smooth functions on $\mathbb{R}^{n}$,

$$
C^{\infty}\left(\mathbb{R}^{n}\right)=\lim _{\longleftarrow} \overline{C^{\infty}\left(\mathbb{R}^{n}\right) / N_{P_{m}}},
$$

where $\left\{K_{m}\right\}_{m \in \mathbb{N}}$ is a sequence of compact subsets of $\mathbb{R}^{n}$ with non-empty interior such that $K_{m}$ is contained in $K_{m+1}$ 's interior and $P_{m}$ is the seminorm defined by

$$
P_{m}(f):=\sup \left\{\left|\partial^{\alpha} f(x)\right|: x \in K_{m},|\alpha| \leq m\right\},
$$

for all $m \in \mathbb{N}$.

2. [53, Example (5), page 25]: The space of all analytic functions on $\mathbb{C}$,

$$
\mathcal{O}(\mathbb{C})=\lim _{\longleftarrow} \overline{\mathcal{O}(\mathbb{C}) / N_{q_{n}}}
$$

where $\mathbb{D}_{n}:=\{z \in \mathbb{C}:|z| \leq 1\}$ and

$$
q_{n}(f):=\sup \left\{|f(z)|: z \in \mathbb{D}_{n}\right\}
$$

for all $n \in \mathbb{N}$.

3. [53, (4), page 35]: The space of continuous functions on $\mathbb{R}^{n}$,

$$
C\left(\mathbb{R}^{n}\right)=\lim _{\longleftarrow} C\left(\mathbb{R}^{n}\right) / N_{p_{K}}=\lim _{\longleftarrow} C(K),
$$

where $K$ runs through the compact subsets of $\mathbb{R}^{n}, p_{k}(f):=\sup \{|f(x)|: x \in K\}$ and $C(K)$ denotes the algebra of all continuous functions on $K$. Note that there was no need to take the closure on each quotient $C\left(\mathbb{R}^{n}\right) / N_{p_{K}}$, in the projective limit above. This is a consequence of the fact that $\left(C\left(\mathbb{R}^{n}\right),\left\{p_{k}\right\}_{k \in \mathbb{N}}\right)$ is a locally $\mathrm{C}^{*}$-algebra see Chapter 3. More generally, if $\mathcal{X}$ is a compactly generated space, ${ }^{35}$ then the conclusions above remain the same.

\footnotetext{
${ }^{34}$ Note that, in view of Lemma 1.7 .1 , the locally convex spaces in the examples are identified with the projective limits in question.

${ }^{35} \mathrm{~A}$ topological space $\mathcal{X}$ is said to be a compactly generated space if, for every subset $B \subseteq \mathcal{X}, B$ is closed if, and only if, $B \cap K$ is closed for each compact subset $K \subseteq \mathcal{X}$.
} 
Besides all of the three examples cited above, the algebras below are also examples of complete Hausdorff locally convex spaces, so the results proved in this section also apply to them:

1. [53, Example (3), page 21]: The algebra $\mathfrak{D}\left(\mathbb{R}^{n}\right)$ of all smooth compactly supported functions on $\mathbb{R}^{n}$ equipped with the inductive limit topology - in other words, a subset $V$ of $\mathfrak{D}\left(\mathbb{R}^{n}\right)$ is a neighborhood of 0 if, and only if, $V \cap \mathfrak{D}_{K_{m}}\left(\mathbb{R}^{n}\right)$ is a neighborhood of 0 in $\mathfrak{D}_{K_{m}}\left(\mathbb{R}^{n}\right)$, for every $m \in \mathbb{N}$, where $K_{m}:=\left\{x \in \mathbb{R}^{n}:\|x\| \leq m+1\right\}$ and

$$
\mathfrak{D}_{K_{m}}\left(\mathbb{R}^{n}\right):=\left\{f \in C^{\infty}\left(\mathbb{R}^{n}\right): \operatorname{supp} f \subseteq K_{m}\right\}
$$

is equipped with the family

$$
\Gamma:=\left\{p_{n}: f \longmapsto \sup \left\{|\partial f(x)|: x \in K_{n},|\alpha| \leq n\right\}\right\}_{n \in \mathbb{N}}
$$

of seminorms (by means of a rescaling argument, which may be found inside the proof of Theorem 3.2, one can obtain a fundamental system of seminorms $\Gamma^{\prime}$ which gives the same topology as $\Gamma$ and turns $\mathfrak{D}_{K_{m}}\left(\mathbb{R}^{n}\right)$ into a Fréchet algebra - see Definition 3.1, Chapter 3).

2. [53, Example (4), page 23]: The algebra $\mathcal{S}\left(\mathbb{R}^{n}\right)$ of all rapidly decreasing functions equipped with the topology defined by the family

$$
\Gamma:=\left\{p_{m, k}: f \longmapsto p_{m, k}(f):=\sup _{x \in \mathbb{R}^{n},|\alpha| \leq m}(1+|x|)^{k}\left|\partial^{\alpha} f(x)\right|\right\}
$$

of seminorms. $\mathcal{S}\left(\mathbb{R}^{n}\right)$ is a Fréchet space and, by a rescaling argument via an application of [90, Proposition 4.3], it can then be shown that $\mathcal{S}\left(\mathbb{R}^{n}\right)$ is a Fréchet algebra, with the appropriate choice of a fundamental system of seminorms.

The five function spaces, above, are examples of complete $\mathrm{m}^{*}$-convex algebras, or ArensMichael *-algebras - such definitions are given in Chapter 3. A concrete example of a complete locally convex $*$-algebra with separately continuous multiplication which is not an Arens-Michael *-algebra (actually, the multiplication is not even jointly continuous), is the following: ${ }^{36}$

The field algebra or Borchers algebra [53, Example 3.10 (5), page 36]:37 Define $\mathcal{S}_{n}:=\mathcal{S}\left(\mathbb{R}^{4 n}\right)$, for $n \in \mathbb{N}, n \geq 1$ and $\mathcal{S}_{0}:=\mathbb{C}$. Consider the pointwise operations of sum and product by scalars on the direct sum

$$
\mathfrak{F}:=\bigoplus_{n=0}^{+\infty} \mathcal{S}_{n}
$$

\footnotetext{
${ }^{36}$ The author would like to thank professor Pedro L. Ribeiro for suggesting the study of this example.

${ }^{37}$ See also the answer given by Pedro L. Ribeiro in https://physics.stackexchange.com/questions/90004/separability-axiom-really-necessary.
} 
and the additional operations of multiplication $(f, g)=\left(\left(f_{n}\right),\left(g_{n}\right)\right) \longmapsto f g:=\left((f g)_{n}\right)_{n \in \mathbb{N}}$, with $(f g)_{0}:=f_{0} g_{0}$,

$$
(f g)_{n}\left(x_{1}, \ldots, x_{n}\right):=\sum_{i+j=n} f_{i}\left(x_{1}, \ldots, x_{i}\right) g_{j}\left(x_{i+1}, \ldots, x_{n}\right), n \geq 1, x_{k} \in \mathbb{R}^{4},
$$

and involution $f=\left(f_{n}\right)_{n \in \mathbb{N}} \longmapsto f^{*}:=\left(f_{n}^{*}\right)$, with

$$
f_{0}^{*}:=\overline{f_{0}}, \quad f_{n}^{*}\left(x_{1}, \ldots x_{n}\right):=\overline{f_{n}\left(x_{n}, x_{n-1}, \ldots, x_{1}\right)}, n \geq 1, x_{k} \in \mathbb{R}^{4} .
$$

Also, equip it with the strict inductive limit topology of the finite sums

$$
\bigoplus_{k=0}^{n} \mathcal{S}_{n}
$$

when these are equipped with the usual product topology - see [72, Proposition 2, page 79]. ${ }^{38}$ Therefore, a subset $V$ of $\mathfrak{F}$ is a neighborhood of 0 if, and only if, $V \cap \oplus_{k=0}^{n} \mathcal{S}_{n}$ is a neighborhood of 0 in $\oplus_{k=0}^{n} \mathcal{S}_{n}$, for every $n \in \mathbb{N}$. Then, with this structure, $\mathfrak{F}$ becomes a barrelled, noncommutative, complete locally convex $*$-algebra with separately continuous multiplication.

\footnotetext{
${ }^{38}$ For the definition of strict inductive limit, see [72, page 84], [117, page 57] or the lecture notes in http://www.math.uni-konstanz.de/ infusino/TVS-II/Lect3.pdf, by Maria Infusino. Note that the definition given in the last two references differs from the one given in [72].
} 

Chapter 2

\section{Group Invariance and Exponentiation}

The first step, which is to construct the maximal $\mathrm{C}^{\infty}$ domain for a fixed finite set of closed linear operators on a complete Hausdorff locally convex space (not necessarily Fréchet), is based on [74, Section 7A, page 151]. The canonical example one should have in mind as an illustration for the construction to follow is the inductive definition, starting from the initial space $C(U)$ of continuous functions on a open set $U \subseteq \mathbb{R}^{d}$, of the spaces $C^{k}(U), k \in \mathbb{N}$, of continuously differentiable functions on $U$, and of the space $C^{\infty}(U)$ of smooth functions on $U$. There, the fixed set of operators is just the set of the usual partial differentiation operators.

\section{Constructing a Group Invariant Domain}

Definition ( $\mathbf{C}^{\infty}$ Domain for a Set of Linear Operators): Let $(\mathcal{X}, \tau)$ be a Hausdorff locally convex space and $\mathcal{S}$ a collection of closable linear operators on $\mathcal{X}$. A subspace $\mathcal{D} \subseteq \mathcal{X}$ satisfying $\mathcal{D} \subseteq$ Dom $A$ and $A[\mathcal{D}] \subseteq \mathcal{D}$, for all $A \in \mathcal{S}$, will be called a $C^{\infty}$ domain for $\mathcal{S}$; moreover, the subspace defined by

$$
\mathcal{X}_{\infty}(\mathcal{S}):=\bigcup\left\{\mathcal{D} \subseteq \mathcal{X}: \mathcal{D} \text { is a } C^{\infty} \text { domain for } \mathcal{S}\right\}
$$

will be called the space of $C^{\infty}$ vectors for $\mathcal{S}$. Note that $\mathcal{X}_{\infty}(\mathcal{S})$ is indeed a vector subspace of $\mathcal{X}$, because if $\mathcal{D}_{1}$ and $\mathcal{D}_{2}$ are $C^{\infty}$ domains for $\mathcal{S}$, then so are $\mathcal{D}_{1}+\mathcal{D}_{2}$ and $\lambda \mathcal{D}_{1}$, for all $\lambda \in \mathbb{C}$. It is clear that $\mathcal{X}_{\infty}(\mathcal{S})$ is also a $C^{\infty}$ domain for $\mathcal{S}$ - it is the maximal $C^{\infty}$ domain for $\mathcal{S}$ with respect to the partial order defined by inclusion.

It may happen, in some cases, that the subspace $\mathcal{X}_{\infty}(\mathcal{S})$ reduces to $\{0\}$, so it is natural to search for sufficient conditions in order to guarantee that this space is "big enough". For the objectives of this manuscript, this "largeness" will amount to two requirements: 
1. $\mathcal{X}_{\infty}(\mathcal{S})$ must be dense in $\mathcal{X}$

2. $\mathcal{X}_{\infty}(\mathcal{S})$ must be a core for each operator $\bar{A}$, where $A \in \mathcal{S}$.

Suppose, now, that $\mathcal{S}:=\left\{B_{j}\right\}_{1 \leq j \leq d}$ is finite and $\mathcal{D} \subseteq \mathcal{X}$ is a $\mathrm{C}^{\infty}$ domain for $\mathcal{S}$, with $\mathcal{X}$ being a complete Hausdorff locally convex space. It will be shown how a more concrete realization of $\mathcal{X}_{\infty}(\overline{\mathcal{S}})$, where

$$
\bar{S}:=\{\bar{B}: B \in \mathcal{S}\}=\left\{\overline{B_{j}}\right\}_{1 \leq j \leq d},
$$

may be obtained, and how to define a natural topology on it. The idea is to proceed via an inductive process to construct a sequence of complete Hausdorff locally convex spaces: let $\Gamma_{0}$ be a family of seminorms on $\mathcal{X}$ which generates its topology and define $\mathcal{X}_{0}:=\mathcal{X}$, $\mathcal{X}_{1}:=\cap_{1 \leq j \leq d}$ Dom $\overline{B_{j}}$ and $B_{0}:=I$. Suppose that there already were constructed $n \geq 1$ Hausdorff locally convex spaces $\left(\mathcal{X}_{k}, \Gamma_{k}\right), 0 \leq k \leq n, \Gamma_{k}:=\left\{\rho_{p, k}: p \in \Gamma\right\}\left(\rho_{p, 0}:=p\right.$, for all $p \in \Gamma)$, in such a way that:

1. $\mathcal{X}_{k+1} \subseteq \mathcal{X}_{k} \subseteq \operatorname{Dom} \overline{B_{j}}$, for $1 \leq j \leq d, 1 \leq k<n$;

2. each $\overline{B_{j}}$ maps $\mathcal{X}_{k+1}$ into $\mathcal{X}_{k}$, for $1 \leq j \leq d, 0 \leq k<n$;

3. $\Gamma_{k+1}:=\left\{\rho_{p, k+1}\right\}_{p \in \Gamma}$, with $\rho_{p, k+1}:=\max \left\{\rho_{p, k}\left(\overline{B_{j}}(\cdot)\right): 0 \leq j \leq d\right\}$, for all $0 \leq k<n$ and all $p \in \Gamma ;^{1}$

4. each $\mathcal{X}_{k}$ is complete with respect to the topology $\tau_{k}$ defined by the family $\Gamma_{k}$ of seminorms, for all $0 \leq k \leq n$ ( $\tau_{0}$ is just the initial topology $\tau$ of $\left.\mathcal{X}\right)$.

Define the subspace $\mathcal{X}_{n+1}:=\left\{x \in \mathcal{X}_{n}: \overline{B_{j}}(x) \in \mathcal{X}_{n}, 1 \leq j \leq d\right\}$ of $\mathcal{X}_{n}$ and a topology $\tau_{n+1}$ on it via a family of seminorms defined by $\Gamma_{n+1}:=\left\{\rho_{p, n+1}\right\}_{p \in \Gamma}$, where

$$
\rho_{p, n+1}:=\max \left\{\rho_{p, n}\left(\overline{B_{j}}(\cdot)\right): 0 \leq j \leq d\right\},
$$

for all $p \in \Gamma$. To see $\left(\mathcal{X}_{n+1}, \tau_{n+1}\right)$ is complete, let $\left\{x_{\alpha}\right\}_{\alpha \in \mathcal{A}}$ be a $\tau_{n+1}$-Cauchy net in $\mathcal{X}_{n+1}$. Then, there exist $x, y \in \mathcal{X}_{n}$ such that $\left\{x_{\alpha}\right\}_{\alpha \in \mathcal{A}} \tau_{n^{-}}$-converges to $x$ and $\left\{\overline{B_{j}}\left(x_{\alpha}\right)\right\}_{\alpha \in \mathcal{A}} \tau_{n^{-}}$ converges to $y$, for all $1 \leq j \leq d$. In particular, $\left\{x_{\alpha}\right\}_{\alpha \in \mathcal{A}} \tau_{0}$-converges to $x$ and $\left\{\overline{B_{j}}\left(x_{\alpha}\right)\right\}_{\alpha \in \mathcal{A}}$ $\tau_{0}$-converges to $y$, for all $1 \leq j \leq d$, so $\left(\tau_{0} \times \tau_{0}\right)$-closedness ${ }^{2}$ of $\overline{B_{j}}, 1 \leq j \leq d$, implies $y=$ $\overline{B_{j}} x$, for all $1 \leq j \leq d$. This proves $x$ belongs to $\left\{z \in \mathcal{X}_{n}: \overline{B_{j}} z \in \mathcal{X}_{n}, 1 \leq j \leq d\right\}=\mathcal{X}_{n+1}$,

\footnotetext{
${ }^{1}$ Here, letting $j$ assume the value 0 is crucial for relating the topologies on $\mathcal{X}_{k}$ and $\mathcal{X}_{k+1}$ : the topology of the latter space is finer than the topology of the former.

${ }^{2}$ The notation " $\left(\tau \times \tau^{\prime}\right)$ " just used, where $\tau$ is the topology of the domain and $\tau^{\prime}$ is the topology of the codomain, was employed to emphasize which topologies are being considered to address the question of closability. A similar notation will be used to deal with continuity of the operators. Sometimes, when the topologies under consideration are the same one ( $\tau$, for example), the more economic terminology " $\tau$-continuity" shall be employed.
} 
so $\left(\mathcal{X}_{n+1}, \tau_{n+1}\right)$ is complete.

The next step will be to show that $\mathcal{X}_{n+1}$ is actually the intersection of the domains of $d\left(\tau_{n} \times \tau_{n}\right)$-closed operators on $\mathcal{X}_{n}$. For each $1 \leq j \leq d$, set $B_{j}^{(n)}:=\left.\left(\overline{B_{j}}\right)\right|_{\mathcal{X}_{n+1}}$ (so Dom $\left.B_{j}^{(n)}:=\mathcal{X}_{n+1}\right)$ and denote by ${\overline{B_{j}^{(n)}}}^{(n)}$ its $\left(\tau_{n} \times \tau_{n}\right)$-closure. ${ }^{3}$ Since the $\tau_{n}$-topology on $\mathcal{X}_{n}$ is finer than the restricted $\tau_{0}$-topology,

$$
\operatorname{Dom} \overline{B_{j}^{(n)}}(n) \subseteq\left\{x \in \mathcal{X}_{n}: \overline{B_{j}} x \in \mathcal{X}_{n}\right\}
$$

because $\left(\mathcal{X}_{n}, \tau_{n}\right)$ is complete. Therefore,

$$
\bigcap_{j=1}^{d} \operatorname{Dom} \overline{B_{j}^{(n)}}(n) \subseteq \bigcap_{j=1}^{d}\left\{x \in \mathcal{X}_{n}: \overline{B_{j}} x \in \mathcal{X}_{n}\right\}=\mathcal{X}_{n+1}
$$

and

$$
\overline{B_{j}^{(n)}}(n) \subset \overline{\left(\overline{B_{j}}\right) \mid \mathcal{X}_{n+1}} \subset \overline{B_{j}}
$$

On the other hand, the definition $\operatorname{Dom} B_{j}^{(n)}:=\mathcal{X}_{n+1}, 1 \leq j \leq d$, trivially implies that $\mathcal{X}_{n+1}$ is contained in

$$
\bigcap_{j=1}^{d} \operatorname{Dom}{\overline{B_{j}^{(n)}}}^{(n)}
$$

Hence, the equality

$$
\bigcap_{j=1}^{d} \operatorname{Dom}{\overline{B_{j}^{(n)}}}^{(n)}=\mathcal{X}_{n+1}
$$

holds.

This concludes the induction step on the construction of a sequence $\left\{\left(\mathcal{X}_{n}, \Gamma_{n}\right)\right\}_{n \in \mathbb{N}}$ of complete Hausdorff locally convex spaces.

Next, consider the subspace

$$
\mathcal{X}_{\infty}:=\bigcap_{n=1}^{+\infty} \mathcal{X}_{n}
$$

equipped with the complete Hausdorff locally convex topology induced by the family of seminorms

$$
\Gamma_{\infty}:=\left\{\rho_{p, n}\right\}_{p \in \Gamma, n \in \mathbb{N}} .
$$

\footnotetext{
${ }^{3}$ Note that it is $\left(\tau_{n} \times \tau_{n}\right)$-closable.
} 
First, note that $\mathcal{X}_{\infty}$ is a $\mathrm{C}^{\infty}$ domain for $\bar{S}$, because $\mathcal{X}_{\infty} \subseteq$ Dom $\overline{B_{j}}$, for every $1 \leq j \leq d$ and, if $x \in \mathcal{X}_{\infty}$, then in particular $x$ belongs to $\mathcal{X}_{n+1}$, for every $n \in \mathbb{N}$, meaning exactly that $\overline{B_{j}} x \in \mathcal{X}_{\infty}$, where $1 \leq j \leq d$. As a consequence,

$$
\mathcal{X}_{\infty} \subseteq \mathcal{X}_{\infty}(\overline{\mathcal{S}})
$$

But, if $x \in \mathcal{X}_{\infty}(\overline{\mathcal{S}})$, then there exists a $\mathrm{C}^{\infty}$ domain $F \subseteq \mathcal{X}$ for $\overline{\mathcal{S}}$ such that $x \in F$. Then, $F \subseteq$ Dom $\overline{B_{j}}$ and $\overline{B_{j}}[F] \subseteq F$, for all $1 \leq j \leq d$, which implies $F \subseteq \mathcal{X}_{1}$ and

$$
F \subseteq\left\{y \in \mathcal{X}_{1}: \overline{B_{j}}(y) \in \mathcal{X}_{1}, 1 \leq j \leq d\right\}=\mathcal{X}_{2} .
$$

Using the fact that $\overline{B_{j}}[F] \subseteq F$, for all $1 \leq j \leq d$, it is seen by the definition of the subspaces $\mathcal{X}_{n}$ and an inductive argument that $F \subseteq \mathcal{X}_{n}$, for all $n \in \mathbb{N}$. Thus

$$
x \in F \subseteq \mathcal{X}_{\infty},
$$

proving the inclusion

$$
\mathcal{X}_{\infty}(\overline{\mathcal{S}}) \subseteq \mathcal{X}_{\infty}
$$

This proves

$$
\mathcal{X}_{\infty}=\mathcal{X}_{\infty}(\overline{\mathcal{S}})
$$

which gives a concrete realization of $\mathcal{X}_{\infty}(\overline{\mathcal{S}})$ as a projective limit of complete Hausdorff locally convex spaces whose topology is furnished by the family $\Gamma_{\infty}$ of seminorms.

The next objective will be to show how to obtain, under the hypotheses fixed above, together with the additional hypothesis of denseness of $\mathcal{D}$ in $\mathcal{X}$, a group invariant dense domain from the fixed dense $\mathrm{C}^{\infty}$ domain $\mathcal{D}$ for $\overline{\mathcal{S}}$. Moreover, it will be proved that this group invariant domain is also a $\mathrm{C}^{\infty}$ domain for $\overline{\mathcal{S}}$.

In the following five theorems some results coming from the theory of linear operators over finite-dimensional complex vector spaces and from the theory of complex Banach algebras will be used. Therefore, sometimes it will be necessary to embed a real Lie algebra $\mathcal{L}$ into its complexification, $\mathcal{L}_{\mathbb{C}}:=\mathcal{L}+i \mathcal{L}$. Define a basis-dependent norm on $\mathcal{L}$ by

$$
\left|\sum_{1 \leq k \leq d} b_{k} B_{k}\right|_{1}:=\sum_{1 \leq k \leq d}\left|b_{k}\right| .
$$

Denoting by $|\cdot| 1, \mathbb{C}$ the norm $\left|\sum_{j=1}^{d} b_{j} B_{j}+c_{j} i B_{j}\right|_{1, \mathbb{C}}:=\sum_{j=1}^{d}\left|b_{j}+i c_{j}\right|$ on the complexified Lie algebra $\mathcal{L}_{\mathbb{C}}$ and by $\|\cdot\|$ the induced operator norm on $\mathcal{L}\left(\mathcal{L}_{\mathbb{C}}\right)$ one sees that $|\cdot|_{1, \mathbb{C}}$ coincides with $|\cdot|_{1}$ on $\mathcal{L}$. Moreover, if $A \in \mathcal{L}$ and ad $A$ denotes the extended linear operator

$$
\tilde{\text { ad }} A: \mathcal{L}_{\mathbb{C}} \longrightarrow \mathcal{L}_{\mathbb{C}}, \quad(\tilde{a d} A)(B+i C):=(\operatorname{ad} A)(B)+i(\operatorname{ad} A)(C),
$$


then

$$
\|\tilde{\operatorname{ad}} A\| \geq\left\|\left.(\tilde{\operatorname{ad}} A)\right|_{\mathcal{L}}\right\|=\|\operatorname{ad} A\| .
$$

Some other definitions which will be of great pertinence are those of augmented spectrum and of diminished resolvent of the closure of a closable operator:

Definition (Augmented Spectrum and Diminished Resolvent): Let $\mathcal{M} \subseteq$ $\operatorname{End}(\mathcal{D})$ be a d-dimensional complex Lie algebra. For each closable $T \in \mathcal{M}$, the linear operator on $\mathcal{M}$ given by ad $T: L \longmapsto($ ad $T)(L):=[T, L]$ has $1 \leq q \leq d$ eigenvalues. They constitute the spectrum $\sigma_{\mathcal{M}}(a d T):=\left\{\mu_{k}\right\}_{1 \leq k \leq q}$ of the linear operator ad $T$ when it is considered as a member of the complex (finite-dimensional) Banach algebra $\mathcal{L}(\mathcal{M})$. In this context, the augmented spectrum of $\overline{\boldsymbol{T}}$ is defined as

$$
\sigma(\bar{T} ; \mathcal{M}):=\sigma(\bar{T}) \cup\left\{\lambda-\mu_{k}: \lambda \in \sigma(\bar{T}), \mu_{k} \in \sigma_{\mathcal{M}}(a d T)\right\} .
$$

Accordingly, the diminished resolvent of $\overline{\boldsymbol{T}}$ is defined as

$$
\rho(\bar{T} ; \mathcal{M}):=\mathbb{C} \backslash \sigma(\bar{T} ; \mathcal{M}) .
$$

If $\mathcal{M}$ is a real Lie algebra and $T \in \mathcal{M}$, then the definitions of augmented spectrum and of diminished resolvent for $\bar{T}$ become, respectively,

$$
\sigma(\bar{T} ; \mathcal{M}):=\sigma(\bar{T}) \cup\left\{\lambda-\mu_{k}: \lambda \in \sigma(\bar{T}), \mu_{k} \in \sigma_{\mathcal{M}_{\mathbb{C}}}(\tilde{a} d T)\right\}
$$

and

$$
\rho(\bar{T} ; \mathcal{M}):=\mathbb{C} \backslash \sigma(\bar{T} ; \mathcal{M})
$$

In order to avoid the repetition of words, the following will be assumed as hypotheses in all of the next four auxiliary theorems (Theorems 2.1, 2.2, 2.3 and 2.4):

Let $(\mathcal{X}, \tau)$ be a complete Hausdorff locally convex space, $\mathcal{D}$ a dense subspace of $\mathcal{X}$ and $\mathcal{L} \subseteq \operatorname{End}(\mathcal{D})$ - remember $\operatorname{End}(\mathcal{D})$ denotes the algebra of all endomorphisms of $\mathcal{D}$ - a finite dimensional real Lie algebra of linear operators, so in particular Dom $B:=\mathcal{D}$, for all $B \in \mathcal{L}$. Fix an ordered basis $\left(B_{k}\right)_{1 \leq k \leq d}$ of $\mathcal{L}$ and define a basis-dependent norm on $\mathcal{L}$ by

$$
\left|\sum_{1 \leq k \leq d} b_{k} B_{k}\right|_{1}:=\sum_{1 \leq k \leq d}\left|b_{k}\right| .
$$

Suppose $\mathcal{L}$ is generated, as a Lie algebra, by a finite set $\mathcal{S}$ of infinitesimal pregenerators of equicontinuous groups and denote by $t \longmapsto V(t, \bar{A})$ the respective one-parameter group generated by $\bar{A}$, for all $A \in \mathcal{S}$. For each $A \in \mathcal{S}$, choose a fundamental system of seminorms 
$\Gamma_{A}$ for $\mathcal{X}$ with respect to which the operator $\bar{A}$ has the $(K I P)$, is $\Gamma_{A}$-conservative and $V(\cdot, \bar{A})$ is $\Gamma_{A}$-isometrically equicontinuous (the arguments at the beginning of Sections 1.3 and 1.4 guarantee the existence of such $\left.\Gamma_{A}\right)$. Define $\Gamma_{A, 1}:=\left\{\rho_{p, 1}\right\}_{p \in \Gamma}$, with $\rho_{p, 1}:=$ $\max \left\{p\left(\overline{B_{j}}(\cdot)\right): 0 \leq j \leq d\right\}$, for all $p \in \Gamma$. Now, assume that the following hypotheses are verified:

1. the basis $\left(B_{k}\right)_{1 \leq k \leq d}$ is formed by closable elements;

2. for each $A \in \mathcal{S}$, there exist two complex numbers $\lambda_{(-, A)}, \lambda_{(+, A)}$ satisfying Re $\lambda_{(-, A)}<$ $-\|\tilde{a d} A\|$ and $\operatorname{Re} \lambda_{(+, A)}>\|\tilde{a} d A\|$, where $\|\tilde{a d} A\|$ denotes the usual operator norm of $\tilde{a d} A: \mathcal{L}_{\mathbb{C}} \longrightarrow \mathcal{L}_{\mathbb{C}}$, such that, for all $\lambda \in \mathbb{C}$ satisfying

$$
|\operatorname{Re} \lambda| \geq M_{A}:=\max \left\{\left|\operatorname{Re} \lambda_{(-, A)}\right|,\left|\operatorname{Re} \lambda_{(+, A)}\right|\right\},
$$

the subspace Ran $(\lambda I-A)$ is dense in $\mathcal{D}$ with respect to the topology $\tau_{1}$ induced by the family $\Gamma_{A, 1}$ (note that asking for this denseness, for a fixed $A \in \mathcal{S}$, is equivalent to asking for $\Gamma_{A^{\prime}, 1}$-denseness, for any other $A^{\prime} \in \mathcal{S}$; this happens because of the fact that $\Gamma_{A}$ is a fundamental system of seminorms for $\mathcal{X}$ - for a more careful argument, see the next paragraph).

As the basis $\left(B_{k}\right)_{1 \leq k \leq d}$ is formed by closable elements, the chain of inclusions

$$
\mathcal{D} \subseteq \bigcap_{k=1}^{d} \operatorname{Dom} \overline{B_{k}} \subseteq \mathcal{X}
$$

is clear. Define $\mathcal{D}_{1}$ as the closure of $\mathcal{D}$ in $\cap_{k=1}^{d}$ Dom $\overline{B_{k}}$ with respect to the $\tau_{1}$-topology. Note that the topology of $\mathcal{D}_{1}$ is independent of the choice of the element $A \in \mathcal{S}$ : let $A_{1}, A_{2} \in \mathcal{S}$ and $\Gamma_{A_{1}, 1}, \Gamma_{A_{2}, 1}$ be their respective families of seminorms, with $\tau_{A_{1}, 1}$ and $\tau_{A_{2}, 1}$ being the corresponding generated topologies. If $x \in \mathcal{D}_{1}$ is a fixed element in the $\tau_{A_{1}, 1}$-closure of $\mathcal{D}$ and $\left\{x_{\alpha}\right\}$ is a net in $\mathcal{D}$ such that $\rho_{p, 1}\left(x_{\alpha}-x\right) \rightarrow 0$, for all $\rho_{p, 1} \in \Gamma_{A_{1}, 1}$, then

$$
p\left(B_{k}\left(x_{\alpha}-x\right)\right) \longrightarrow 0, \quad p \in \Gamma_{A_{1}}, 0 \leq k \leq d .
$$

Since $\Gamma_{A_{1}}$ is a fundamental system of seminorms for $\mathcal{X}$, this is equivalent to the convergence $B_{k}\left(x_{\alpha}-x\right) \rightarrow 0$ in $\mathcal{X}$, for each $0 \leq k \leq d$. But, again, $\Gamma_{A_{2}}$ is also a fundamental system of seminorms for $\mathcal{X}$, so

$$
p^{\prime}\left(B_{k}\left(x_{\alpha}-x\right)\right) \longrightarrow 0, \quad p^{\prime} \in \Gamma_{A_{2}}, 0 \leq k \leq d,
$$

must hold. But this implies $x$ belongs to the $\tau_{A_{2}, 1^{-}}$closure of $\mathcal{D}$, showing that the $\tau_{A_{1}, 1^{-}}$ closure of $\mathcal{D}$ is contained in the $\tau_{A_{2}, 1^{-}}$-closure. By symmetry, this implies that they are equal, showing the asserted independence. 
Definition $\left(\mathbf{C}^{\mathbf{1}}\right.$-closure of $\left.\mathcal{D}\right)$ : For the rest of this chapter the topology on $\mathcal{D}_{1}$ generated by the family $\Gamma_{A, 1}$, for some $A \in \mathcal{S}$, will be denoted by $\boldsymbol{\tau}_{\mathbf{1}}$.

Note, also, the inclusions

$$
\mathcal{D} \subseteq \mathcal{D}_{1} \subseteq \bigcap_{1 \leq k \leq d} \operatorname{Dom} \overline{B_{k}}
$$

The next theorem is a straightforward adaptation of [74, Theorem 5.1, page 112].

Theorem 2.1 (Commutation Relations Involving the Resolvent Operators): Let $A \in \mathcal{S}$ with $\sigma_{\mathcal{L}_{\mathbb{C}}}(\tilde{a} d A):=\left\{\mu_{j}\right\}_{1 \leq j \leq q}$. If $\lambda \in \rho(\bar{A} ; \mathcal{L})$ and $\mu \in \mathbb{C}$ is a number such that $\lambda+\mu \in \rho(\bar{A})$, then

$$
\begin{gathered}
B R(\lambda, \bar{A})(x)=\sum_{k=0}^{n}(-1)^{k} R(\lambda+\mu, \bar{A})^{k+1}(a d A-\mu)^{k}(B)(x) \\
+(-1)^{n+1} R(\lambda+\mu, \bar{A})^{n+1}(a d A-\mu)^{n+1}(B) R(\lambda, \bar{A})(x),
\end{gathered}
$$

where $x \in \operatorname{Ran}(\lambda I-A), B \in \mathcal{L}$ and $n \in \mathbb{N}$. Also,

$$
\begin{gathered}
(2.1 .2) \quad B R(\lambda, \bar{A})(x) \\
\sum_{1 \leq j \leq q, 0 \leq k \leq s_{j}}(-1)^{k} R\left(\lambda+\mu_{j}, \bar{A}\right)^{k+1}\left(a d A-\mu_{j} I\right)^{k}\left(P_{j} B\right)(x), \quad x \in \operatorname{Ran}(\lambda I-A), B \in \mathcal{L},
\end{gathered}
$$

with $P_{j}$ being the projection over the generalized eigenspace

$$
\left(\mathcal{L}_{\mathbb{C}}\right)_{j}:=\left\{B \in \mathcal{L}_{\mathbb{C}}:\left(\tilde{a} d A-\mu_{j} I\right)^{s}(B)=0, \text { for some } s\right\}
$$

and $s_{j}$ being the non-negative integer satisfying $\left(\tilde{a} d A-\mu_{j} I\right)^{s_{j}} \neq 0=\left(\tilde{a d} A-\mu_{j} I\right)^{s_{j}+1}$ on $\left(\mathcal{L}_{\mathbb{C}}\right)_{j} \cdot{ }^{4}$

Proof of Theorem 2.1: To prove (2.1.1), first note that if $x=(\lambda I-A)(y)$ and $B \in \mathcal{L}$,

$$
\begin{gathered}
\mathrm{R}(\lambda+\mu, \bar{A}) B(x)=\mathrm{R}(\lambda+\mu, \bar{A}) B(\lambda I-A)(y) \\
=\mathrm{R}(\lambda+\mu, \bar{A})[(\lambda+\mu-A) B+[A, B]-\mu B](y)=B(y)+\mathrm{R}(\lambda+\mu, \bar{A})(\operatorname{ad} A-\mu)(B)(y) \\
=B \mathrm{R}(\lambda, \bar{A})(x)+\mathrm{R}(\lambda+\mu, \bar{A})(\operatorname{ad} A-\mu)(B) \mathrm{R}(\lambda, \bar{A})(x) .
\end{gathered}
$$

\footnotetext{
${ }^{4}$ The existence of the projections $P_{j}$ and the integers $s_{j}$ is guaranteed by the Primary Decomposition Theorem - see [69, Theorem 12, page 220].
} 
Now, suppose that for a fixed $n \in \mathbb{N}$, the equality

$$
\begin{gathered}
C \mathrm{R}(\lambda, \bar{A})(x)=\sum_{k=0}^{n}(-1)^{k} \mathrm{R}(\lambda+\mu, \bar{A})^{k+1}(\operatorname{ad} A-\mu)^{k}(C)(x) \\
+(-1)^{n+1} \mathrm{R}(\lambda+\mu, \bar{A})^{n+1}(\operatorname{ad} A-\mu)^{n+1}(C) \mathrm{R}(\lambda, \bar{A})(x), \quad x \in \operatorname{Ran}(\lambda I-A),
\end{gathered}
$$

holds, for all $C \in \mathcal{L}$. Substituting $C$ by $(\operatorname{ad} A-\mu)(B)$, with $B \in \mathcal{L}$, one obtains

$$
\begin{gathered}
(\operatorname{ad} A-\mu)(B) \mathrm{R}(\lambda, \bar{A})(x)=\sum_{k=0}^{n}(-1)^{k} \mathrm{R}(\lambda+\mu, \bar{A})^{k+1}(\operatorname{ad} A-\mu)^{k+1}(B)(x) \\
+(-1)^{n+1} \mathrm{R}(\lambda+\mu, \bar{A})^{n+1}(\operatorname{ad} A-\mu)^{n+2}(B) \mathrm{R}(\lambda, \bar{A})(x), \quad x \in \operatorname{Ran}(\lambda I-A) .
\end{gathered}
$$

Applying the operator $\mathrm{R}(\lambda+\mu, \bar{A})$ on both members and using the first equality obtained in the proof gives

$$
\begin{gathered}
\mathrm{R}(\lambda+\mu, \bar{A}) B(x)-B \mathrm{R}(\lambda, \bar{A})(x)=\mathrm{R}(\lambda+\mu, \bar{A})(\operatorname{ad} A-\mu)(B) \mathrm{R}(\lambda, \bar{A})(x) \\
=\sum_{k=0}^{n}(-1)^{k} \mathrm{R}(\lambda+\mu, \bar{A})^{k+2}(\operatorname{ad} A-\mu)^{k+1}(B)(x) \\
+(-1)^{n+1} \mathrm{R}(\lambda+\mu, \bar{A})^{n+2}(\operatorname{ad} A-\mu)^{n+2}(B) \mathrm{R}(\lambda, \bar{A})(x), \quad x \in \operatorname{Ran}(\lambda I-A), B \in \mathcal{L} .
\end{gathered}
$$

This yields

$$
\begin{gathered}
B \mathrm{R}(\lambda, \bar{A})(x)=\sum_{k=0}^{n+1}(-1)^{k} \mathrm{R}(\lambda+\mu, \bar{A})^{k+1}(\operatorname{ad} A-\mu)^{k}(B)(x) \\
+(-1)^{n+2} \mathrm{R}(\lambda+\mu, \bar{A})^{n+2}(\operatorname{ad} A-\mu)^{n+2} \mathrm{R}(\lambda, \bar{A})(x)
\end{gathered}
$$

for all $x \in \operatorname{Ran}(\lambda I-A)$ and $B \in \mathcal{L}$, which finishes the induction proof and establishes (2.1.1) for all $n \in \mathbb{N}$.

In order to prove (2.1.2), first note that $\lambda+\mu$ belongs to $\rho(\bar{A})$ whenever $\mu$ belongs to $\sigma_{\mathcal{L}_{\mathbb{C}}}(\tilde{a d} A)$. Indeed, if $\lambda+\mu$ belonged to $\sigma(\bar{A})$, then $\lambda=(\lambda+\mu)-\mu \in \sigma\left(\bar{A} ; \mathcal{L}_{\mathbb{C}}\right)$, which is absurd. Therefore, if $\mu \in \sigma_{\mathcal{L}_{\mathbb{C}}}(\tilde{\sim}$ ad $A),(2.1 .1)$ may be applied for $\lambda+\mu$. Substituting $B \in \mathcal{L}$ by $P_{j} B \in \mathcal{L},{ }^{5} \mu$ by $\mu_{j} \in \sigma_{\mathcal{L}_{\mathbb{C}}}(\tilde{a d} A)$ and $n$ by $s_{j},(2.1 .1)$ gives

$$
\begin{gathered}
P_{j} B \mathrm{R}(\lambda, \bar{A})(x)=\sum_{k=0}^{s_{j}}(-1)^{k} \mathrm{R}\left(\lambda+\mu_{j}, \bar{A}\right)^{k+1}\left(\operatorname{ad} A-\mu_{j}\right)^{k}\left(P_{j} B\right)(x) \\
\quad+(-1)^{s_{j}+1} \mathrm{R}\left(\lambda+\mu_{j}, \bar{A}\right)^{s_{j}+1}\left(\operatorname{ad} A-\mu_{j}\right)^{s_{j}+1}\left(P_{j} B\right) \mathrm{R}(\lambda, \bar{A})(x)
\end{gathered}
$$

\footnotetext{
${ }^{5} P_{j} B \in \mathcal{L}$ follows from the fact that $P_{j}$ is a polynomial in ad $A$.
} 


$$
=\sum_{k=0}^{s_{j}}(-1)^{k} \mathrm{R}\left(\lambda+\mu_{j}, \bar{A}\right)^{k+1}\left(\operatorname{ad} A-\mu_{j}\right)^{k}\left(P_{j} B\right)(x), \quad x \in \operatorname{Ran}(\lambda I-A), B \in \mathcal{L},
$$

for all $\mu_{j} \in \sigma_{\mathcal{L}_{\mathbb{C}}}(\tilde{a d} A)$. Since $\sum_{1 \leq j \leq q} P_{j} B=B$, summation of the last equation over all $1 \leq j \leq q$ establishes the desired result.

From Theorem 2.2 until Theorem 2.4, consider fixed an $A \in \mathcal{S}$ with

$$
\sigma_{\mathcal{L}_{\mathrm{C}}}(\tilde{\operatorname{ad}} \boldsymbol{A}):=\left\{\mu_{j}\right\}_{1 \leq j \leq q} .
$$

It is also important to have in mind that the results in all of the next three theorems - and even in Theorem 2.1 - remain valid if one substitutes $A$ by $-A$. This observation will be particularly useful in Theorem 2.4.

Theorem 2.2 below is based on [74, Theorem 5.4(1), page 119], but here it is assumed that $A$ is not just closable, but that it is also $\Gamma_{A}$-conservative. This conservativity hypothesis (along with the (KIP)) will be, just as in Theorems 2.3 and 2.4, a key hypothesis to circumvent the fact that the resolvent operator $\mathrm{R}(\lambda, \bar{A})$ does not belong, in general, to a Banach algebra of operators on $\mathcal{X}$. This is a technical obstruction which makes it difficult to extend the results beyond the normed case, as noted in [74, page 113]. In the more general setting of complete Hausdorff locally convex spaces, one has the following:

Theorem 2.2 ( $\mathbf{C}^{1}$-Continuity of the Resolvent Operators): Let $\lambda \in \mathbb{C}$ satisfy $|\operatorname{Re} \lambda| \geq M_{A}:=\max \left\{\left|\operatorname{Re} \lambda_{(-, A)}\right|,\left|\operatorname{Re} \lambda_{(+, A)}\right|\right\}>0$ so that, in particular, $\lambda \in \rho(\bar{A})$, by Lemma 1.3.1. Then, $R(\lambda, \bar{A})$ leaves $\mathcal{D}_{1}$ invariant and restricts there as a $\tau_{1}$-continuous linear operator $R_{1}(\lambda, \bar{A})$. Moreover, $R_{1}(\lambda, \bar{A})=\left(\lambda I-\bar{A}^{\tau_{1}}\right)^{-1}=R\left(\lambda, \bar{A}^{\tau_{1}}\right)$, where $\bar{A}^{\tau_{1}}$ denotes the $\left(\tau_{1} \times \tau_{1}\right)$-closure of the operator $A: \mathcal{D} \subseteq \mathcal{D}_{1} \longrightarrow \mathcal{D}_{1}$ when seen as a densely defined linear operator acting on $\mathcal{D}_{1}$.

Proof of Theorem 2.2: First note that the condition

$$
|\operatorname{Re} \lambda| \geq M_{A}:=\max \left\{\left|\operatorname{Re} \lambda_{(-, A)}\right|,\left|\operatorname{Re} \lambda_{(+, A)}\right|\right\}
$$

implies $\lambda \in \rho(\bar{A} ; \mathcal{L})$, so Theorem 2.1 is applicable. By equation (2.1.2) of Theorem 2.1,

$$
\begin{gathered}
B_{k} \mathrm{R}(\lambda, \bar{A})(x) \\
=\sum_{1 \leq j \leq q, 0 \leq i \leq s_{j}}(-1)^{i} \mathrm{R}\left(\lambda+\mu_{j}, \bar{A}\right)^{i+1}\left(\operatorname{ad} A-\mu_{j} I\right)^{i}\left(P_{j} B_{k}\right)(x),
\end{gathered}
$$

for every $1 \leq k \leq d$ and $x \in \operatorname{Ran}(\lambda I-A)$, with $P_{j}$ being the projection over the generalized eigenspace

$$
\left(\mathcal{L}_{\mathbb{C}}\right)_{j}:=\left\{B \in \mathcal{L}_{\mathbb{C}}:\left(\tilde{a d} A-\mu_{j} I\right)^{s}(B)=0, \text { for some } s\right\}
$$


and $s_{j}$ being the non-negative integer satisfying $\left(\tilde{\operatorname{ad}} A-\mu_{j} I\right)^{s_{j}} \neq 0=\left(\tilde{a d} A-\mu_{j} I\right)^{s_{j}+1}$ on $\left(\mathcal{L}_{\mathbb{C}}\right)_{j}$. The restriction $|\operatorname{Re} \lambda|>\|\tilde{a d} A\|_{\mathbb{C}}$ of the hypothesis implies $\lambda+\mu_{j} \neq 0$, for all $1 \leq j \leq q$, because $\left|\mu_{j}\right| \leq\|\tilde{a d} A\|_{\mathbb{C}}$, so $\operatorname{Re} \lambda+\operatorname{Re} \mu_{j} \neq 0$. Since $\bar{A}$ is a $\Gamma_{A}$-conservative linear operator, it follows that

$$
p\left(\left(\left(\lambda+\mu_{j}\right) I-\bar{A}\right)(x)\right) \geq\left|\lambda+\mu_{j}\right| p(x), \quad p \in \Gamma_{A}, x \in \operatorname{Dom} \bar{A} .
$$

Therefore, in particular,

$$
p\left(\mathrm{R}\left(\lambda+\mu_{j}, \bar{A}\right)(x)\right) \leq \frac{1}{\left|\lambda+\mu_{j}\right|} p(x), \quad p \in \Gamma_{A}, x \in \mathcal{X} .
$$

Hence, $\mathrm{R}\left(\lambda+\mu_{j}, \bar{A}\right)$ possesses the (KIP) with respect to $\Gamma_{A}$, for all $1 \leq j \leq q$ - see Section 1.4. This allows one to define for each $p \in \Gamma_{A}$ and each $1 \leq j \leq q$ the unique everywhere-defined continuous extension of the densely defined $\|\cdot\|_{p}$-continuous linear operator $\left(\mathrm{R}\left(\lambda+\mu_{j}, \bar{A}\right)\right)_{p}$ induced by $\mathrm{R}\left(\lambda+\mu_{j}, \bar{A}\right)$ on the completion $\mathcal{X}_{p}$ - see Lemma 1.6.1. Such extension will be denoted by $\mathrm{R}_{p}\left(\lambda+\mu_{j}, \bar{A}\right)$, for every $p \in \Gamma_{A}$ and $1 \leq j \leq q$. Analogously, the $\|\cdot\|_{p^{-}}$-continuous linear operator $(\mathrm{R}(\lambda, \bar{A}))_{p}$ also has such an extension $\mathrm{R}_{p}(\lambda, \bar{A})$ on $\mathcal{X}_{p}$, by the $\Gamma_{A}$-conservativity hypothesis.

For each $1 \leq k \leq d, 1 \leq j \leq q$ and $0 \leq i \leq s_{j}$ write

$$
\left(\operatorname{ad} A-\mu_{j} I\right)^{i}\left(P_{j} B_{k}\right)=\sum_{l=1}^{d} c_{l} B_{l}, \quad c_{l} \in \mathbb{R}^{6}
$$

Then, for all $x \in \mathcal{D}$ the estimates

$$
\begin{gathered}
p\left(\left(\operatorname{ad} A-\mu_{j} I\right)^{i}\left(P_{j} B_{k}\right)(x)\right) \leq \sum_{l=1}^{d}\left|c_{l}\right| p\left(B_{l}(x)\right) \\
\leq\left|\left(\operatorname{ad} A-\mu_{j} I\right)^{i}\left(P_{j} B_{k}\right)\right|_{1} \max \left\{p\left(B_{k}(x)\right): 0 \leq k \leq d\right\} \\
\leq\left\|\tilde{a d} A-\mu_{j} I\right\|^{i}\left|P_{j} B_{k}\right|_{1} \rho_{p, 1}(x), \quad p \in \Gamma_{A},
\end{gathered}
$$

are legitimate. Therefore, denoting by $\|\cdot\|_{p}$ the usual operator norm on $\mathcal{L}\left(\mathcal{X}_{p}\right)$, the inequality

$$
\begin{gathered}
p\left(B_{k} \mathrm{R}(\lambda, \bar{A})(x)\right)=p\left(\sum_{1 \leq j \leq q, 0 \leq i \leq s_{j}}(-1)^{i} \mathrm{R}\left(\lambda+\mu_{j}, \bar{A}\right)^{i+1}\left(\operatorname{ad} A-\mu_{j} I\right)^{i}\left(P_{j} B_{k}\right)(x)\right) \\
\leq \sum_{1 \leq j \leq q, 0 \leq i \leq s_{j}} p\left(\mathrm{R}_{p}\left(\lambda+\mu_{j}, \bar{A}\right)^{i+1}\left[\left(\operatorname{ad} A-\mu_{j} I\right)^{i}\left(P_{j} B_{k}\right)(x)\right]_{p}\right)
\end{gathered}
$$

\footnotetext{
${ }^{6}$ This is possible because $A$ and $P_{j} B_{k}$ both belong to $\mathcal{L}$.
} 


$$
\begin{aligned}
& \leq \sum_{1 \leq j \leq q, 0 \leq i \leq s_{j}}\left\|\mathrm{R}_{p}\left(\lambda+\mu_{j}, \bar{A}\right)\right\|_{p}^{i+1} p\left(\left(\operatorname{ad} A-\mu_{j} I\right)^{i}\left(P_{j} B_{k}\right)(x)\right) \\
& \leq \sum_{1 \leq j \leq q, 0 \leq i \leq s_{j}}\left\|\mathrm{R}_{p}\left(\lambda+\mu_{j}, \bar{A}\right)\right\|_{p}^{i+1}\left\|\tilde{\operatorname{ad}} A-\mu_{j} I\right\|^{i}\left|P_{j} B_{k}\right|_{1} \rho_{p, 1}(x) \\
& \leq C_{p}(k) \rho_{p, 1}(x), \quad p \in \Gamma_{A}, 1 \leq k \leq d, x \in \operatorname{Ran}(\lambda I-A)
\end{aligned}
$$

is obtained, for some $C_{p}(k)>0$. For $k=0$ the estimate with $B_{0}=I$ is simpler:

$$
p\left(B_{0} \mathrm{R}(\lambda, \bar{A})(x)\right) \leq\left\|\mathrm{R}_{p}(\lambda, \bar{A})\right\|_{p} p(x) \leq\left\|\mathrm{R}_{p}(\lambda, \bar{A})\right\|_{p} \rho_{p, 1}(x), \quad p \in \Gamma_{A},
$$

where $\mathrm{R}_{p}(\lambda, \bar{A})$ is defined in an analogous way as the operators $\mathrm{R}_{p}\left(\lambda+\mu_{j}, \bar{A}\right)$ were. This implies, upon taking the maximum over $0 \leq k \leq d$, that

$$
\begin{gathered}
\rho_{p, 1}(\mathrm{R}(\lambda, \bar{A})(x)) \\
\leq \max \left\{\left\|\mathrm{R}_{p}(\lambda, \bar{A})\right\|_{p}, \max \left\{C_{p}(k): 1 \leq k \leq d\right\}\right\} \rho_{p, 1}(x), \quad p \in \Gamma_{A}, x \in \operatorname{Ran}(\lambda I-A),
\end{gathered}
$$

showing that $\mathrm{R}(\lambda, \bar{A}): \operatorname{Ran}(\lambda I-A) \longrightarrow \mathcal{D} \subseteq \mathcal{D}_{1}$ is a $\tau_{1}$-continuous linear operator on $\mathcal{D}_{1}$. Hence, one might extend it via limits to a linear $\tau_{1}$-continuous everywhere defined linear operator $\mathrm{R}_{1}(\lambda, \bar{A})$ on $\mathcal{D}_{1}$, by Lemma 1.6.1, as a consequence of the $\tau_{1}$-denseness hypothesis of $\operatorname{Ran}(\lambda I-A)$ in $\mathcal{D}$. Since $\tau_{1}$ is finer than $\tau$ and $\mathrm{R}(\lambda, \bar{A})$ is $\tau$-continuous, the equality

$$
\mathrm{R}_{1}(\lambda, \bar{A})=\left.\mathrm{R}(\lambda, \bar{A})\right|_{\mathcal{D}_{1}}
$$

is true and, moreover, $\mathrm{R}(\lambda, \bar{A})$ leaves $\mathcal{D}_{1}$ invariant (to obtain this conclusion, it was also used that $\mathcal{D}_{1}$ is Hausdorff with respect to its $\tau_{1}$-topology).

To prove $\mathrm{R}_{1}(\lambda, \bar{A})=\left(\lambda I-\bar{A}^{\tau_{1}}\right)^{-1}$ first note that, in fact, $A$ is a $\left(\tau_{1} \times \tau_{1}\right)$-closable linear operator: indeed, if $\left\{x_{\alpha}\right\}$ is a net in $\mathcal{D}$ which is $\tau_{1}$-convergent to 0 and $A\left(x_{\alpha}\right) \stackrel{\alpha}{\rightarrow} y \in \mathcal{D}_{1}$, then both nets $\left\{x_{\alpha}\right\}$ and $\left\{A\left(x_{\alpha}\right)\right\}$ are $\tau$-convergent to 0 and $y$, respectively, since the $\tau_{1^{-}}$ topology is stronger than the $\tau$-topology. Since $A$ is $(\tau \times \tau)$-closable, it follows that $y=0$, which proves $A$ is $\left(\tau_{1} \times \tau_{1}\right)$-closable. Hence, its $\left(\tau_{1} \times \tau_{1}\right)$-closure, $\bar{A}^{\tau_{1}}$, is well-defined, and $\bar{A}^{\tau_{1}} \subseteq \bar{A}$.

Now, fix $x \in \operatorname{Dom} \bar{A}^{\tau_{1}}$ and take a net $\left\{x_{\alpha}\right\}$ in $\mathcal{D}$ such that $x_{\alpha} \longrightarrow x$ and $A\left(x_{\alpha}\right) \longrightarrow$ $\bar{A}^{\tau_{1}}(x)$, both convergences being in the $\tau_{1}$-topology. This implies

$$
\mathrm{R}_{1}(\lambda, \bar{A})\left[\left(\lambda I-\bar{A}^{\tau_{1}}\right)\left(x_{\alpha}\right)\right]=\mathrm{R}(\lambda, \bar{A})\left[(\lambda I-A)\left(x_{\alpha}\right)\right]=x_{\alpha} \longrightarrow x
$$

in the $\tau_{1}$-topology. But

$$
\left(\lambda I-\bar{A}^{\tau_{1}}\right)\left(x_{\alpha}\right)=(\lambda I-A)\left(x_{\alpha}\right) \longrightarrow\left(\lambda I-\bar{A}^{\tau_{1}}\right)(x)
$$

relatively to the $\tau_{1}$-topology, which implies

$$
\mathrm{R}_{1}(\lambda, \bar{A})\left[\left(\lambda I-\bar{A}^{\tau_{1}}\right)\left(x_{\alpha}\right)\right] \longrightarrow \mathrm{R}_{1}(\lambda, \bar{A})\left[\left(\lambda I-\bar{A}^{\tau_{1}}\right)(x)\right]
$$


in the $\tau_{1}$-sense, by $\tau_{1}$-continuity of the linear operator $\mathrm{R}_{1}(\lambda, \bar{A})$. By the uniqueness of the limit,

$$
\mathrm{R}_{1}(\lambda, \bar{A})\left[\left(\lambda I-\bar{A}^{\tau_{1}}\right)(x)\right]=x .
$$

This proves that $\mathrm{R}_{1}(\lambda, \bar{A})$ is a left inverse for $\left(\lambda I-\bar{A}^{\tau_{1}}\right)$. To prove that $\mathrm{R}_{1}(\lambda, \bar{A})$ is a right inverse, fix an arbitrary $x \in \mathcal{D}_{1}$ and a net $\left\{x_{\alpha}\right\}$ in $\mathcal{D}$ such that $x_{\alpha} \longrightarrow x$ in the $\tau_{1}$-topology. Then,

$$
\left(\lambda I-\bar{A}^{\tau_{1}}\right) \mathrm{R}_{1}(\lambda, \bar{A})\left(x_{\alpha}\right)=(\lambda I-A) \mathrm{R}(\lambda, \bar{A})\left(x_{\alpha}\right)=x_{\alpha} \longrightarrow x,
$$

in the $\tau_{1}$-topology. On the other hand, $\tau_{1}$-continuity of $\mathrm{R}_{1}(\lambda, \bar{A})$ and $\tau_{1}$-closedness of $\bar{A}^{\tau_{1}}$ imply

$$
\left(\lambda I-\bar{A}^{\tau_{1}}\right) \mathrm{R}_{1}(\lambda, \bar{A})\left(x_{\alpha}\right) \longrightarrow\left(\lambda I-\bar{A}^{\tau_{1}}\right) \mathrm{R}_{1}(\lambda, \bar{A})(x) .
$$

As before, uniqueness of the limit establishes that $\mathrm{R}_{1}(\lambda, \bar{A})$ is a right inverse for $\lambda I-\bar{A}^{\tau_{1}}$. This proves $\mathrm{R}_{1}(\lambda, \bar{A})$ is a two-sided inverse for the operator $\lambda I-\bar{A}^{\tau_{1}}$ on $\mathcal{D}_{1}$ and that $\mathrm{R}_{1}(\lambda, \bar{A})=\mathrm{R}\left(\lambda, \bar{A}^{\tau_{1}}\right)$, as claimed.

The proof of the next theorem is strongly inspired in that of [74, Theorem 5.2, page 114], and gives a version of formula (1) of the reference in the context of locally convex spaces, when $A$ is assumed to be a pregenerator of an equicontinuous group.

Theorem 2.3 (Series Expansion of Commutation Relations): Let $\lambda \in \mathbb{C}$ satisfy

$$
|\operatorname{Re} \lambda| \geq M_{A}:=\max \left\{\left|\operatorname{Re} \lambda_{(-, A)}\right|,\left|\operatorname{Re} \lambda_{(+, A)}\right|\right\}>0 \text {. }
$$

For all $x \in \mathcal{D}$ and all closable $B \in \mathcal{L}$, it is verified that $R(\lambda, \bar{A})(x) \in \operatorname{Dom} \bar{B}$ and

$$
\bar{B} R(\lambda, \bar{A})(x)=\sum_{k=0}^{+\infty}(-1)^{k} R(\lambda, \bar{A})^{k+1}\left[(a d A)^{k}(B)\right](x) .
$$

Proof of Theorem 2.3: By (2.1.1) of Theorem 2.1,

$$
\begin{gathered}
B \mathrm{R}(\lambda, \bar{A})(x)=\left[\sum_{k=0}^{n}(-1)^{k} \mathrm{R}(\lambda, \bar{A})^{k+1}(\operatorname{ad} A)^{k}(B)(x)\right] \\
+(-1)^{n+1} \mathrm{R}(\lambda, \bar{A})^{n+1}(\operatorname{ad} A)^{n+1}(B) \mathrm{R}(\lambda, \bar{A})(x),
\end{gathered}
$$

for all $n \in \mathbb{N}, x \in \operatorname{Ran}(\lambda I-A)$ and $B \in \mathcal{L}$. In order to prove that the remainder

$$
(-1)^{n+1} \mathrm{R}(\lambda, \bar{A})^{n+1}(\operatorname{ad} A)^{n+1}(B) \mathrm{R}(\lambda, \bar{A})(x)
$$

goes to 0 , as $n \longrightarrow+\infty$, it will be shown the existence of an $M>0$ with the property that, for all $p \in \Gamma_{A}$, there exists $0<r_{p}<1$ in a way that

$$
p\left(\mathrm{R}(\lambda, \bar{A})^{k}(\operatorname{ad} A)^{k}(B) x\right) \leq M r_{p}^{k} \rho_{p, 1}(x), \quad k \in \mathbb{N}, x \in \mathcal{D} .
$$


To this purpose, it will be necessary to embed $\mathcal{L}$ into its complexification, $\mathcal{L}_{\mathbb{C}}:=\mathcal{L}+i \mathcal{L}$, since results coming from the complex Banach algebras' realm will be used. Denote by $\mathrm{R}_{p}(\lambda, \bar{A})$, as in Theorem 2.2, the unique everywhere-defined continuous extension of the

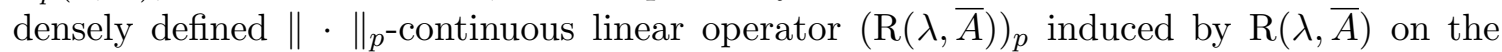
completion $\mathcal{X}_{p}$. Since for each fixed $1 \leq k \leq d$,

$$
(\operatorname{ad} A)^{k}(B)=\sum_{l=1}^{d} c_{l}^{(k)} B_{l}, \quad c_{l} \in \mathbb{R},
$$

one obtains

$$
\begin{gathered}
p\left(\mathrm{R}(\lambda, \bar{A})^{k}(\operatorname{ad} A)^{k}(B) x\right)=\left\|\mathrm{R}_{p}(\lambda, \bar{A})^{k}\left[(\operatorname{ad} A)^{k}(B) x\right]_{p}\right\|_{p} \\
\leq\left\|\mathrm{R}_{p}(\lambda, \bar{A})^{k}\right\|_{p}\left\|\left[(\operatorname{ad} A)^{k}(B) x\right]_{p}\right\|_{p}=\left\|\mathrm{R}_{p}(\lambda, \bar{A})^{k}\right\|_{p} p\left((\operatorname{ad} A)^{k}(B) x\right) \\
\leq\left\|\mathrm{R}_{p}(\lambda, \bar{A})^{k}\right\|_{p}\left|(\operatorname{ad} A)^{k}(B)\right|_{1} \rho_{p, 1}(x) \leq\left\|\mathrm{R}_{p}(\lambda, \bar{A})^{k}\right\|_{p}\left\|(\tilde{\operatorname{ad}} A)^{k}\right\||B|_{1} \rho_{p, 1}(x),
\end{gathered}
$$

for all $x \in \mathcal{D}$ - note that the majoration of $p\left((\operatorname{ad} A)^{k}(B) x\right)$ is obtained in a similar way of that done in the proof of Theorem 2.2. Making $M:=|B|_{1}$, it is sufficient to show that for some $0<r_{p}<1$ and for all sufficiently large $k$,

$$
\left\|\mathrm{R}_{p}(\lambda, \bar{A})^{k}\right\|_{p}\left\|(\tilde{\operatorname{ad}} A)^{k}\right\|<r_{p}^{k}
$$

or, equivalently,

$$
\left\|\mathrm{R}_{p}(\lambda, \bar{A})^{k}\right\|_{p}^{1 / k}\left\|(\tilde{a d} A)^{k}\right\|^{1 / k}<r_{p}
$$

Since $\mathcal{L}\left(\mathcal{X}_{p}\right)$ and $\mathcal{L}\left(\mathcal{L}_{\mathbb{C}}\right)$ are both complex unital Banach algebras, by Gelfand's spectral radius formula it is sufficient to show that the product of

$$
\nu\left(\mathrm{R}_{p}(\lambda, \bar{A})\right):=\lim _{k \rightarrow+\infty}\left\|\mathrm{R}_{p}(\lambda, \bar{A})^{k}\right\|_{p}^{1 / k}
$$

and

$$
\nu(\tilde{\operatorname{ad}} A):=\lim _{k \rightarrow+\infty}\left\|(\tilde{\operatorname{ad}} A)^{k}\right\|^{1 / k}
$$

is strictly less than 1. By Lemma 1.4.1 and [50, 3.5 Generation Theorem (contraction case), page 73] (whose full statement was already exposed in Section 1.2), the linear operator $(\bar{A})_{p}$ is closable and the resolvent of its closure satisfies the norm inequality

$$
\left\|\mathrm{R}\left(\lambda, \overline{(\bar{A})_{p}}\right)\right\|_{p} \leq \frac{1}{|\operatorname{Re} \lambda|}
$$

since $\overline{(\bar{A})_{p}}$ generates a strongly continuous group of isometries on $\mathcal{X}_{p}$. It will now be proved that

$$
\mathrm{R}\left(\lambda, \overline{(\bar{A})_{p}}\right)=\mathrm{R}_{p}(\lambda, \bar{A}) .
$$


So fix $x_{p} \in \operatorname{Dom} \overline{(\bar{A})_{p}}$ and take a net $\left\{\left[x_{\alpha}\right]_{p}\right\}$ in $\operatorname{Dom}(\bar{A})_{p}$ such that

$$
\left[x_{\alpha}\right]_{p} \stackrel{\alpha}{\rightarrow} x_{p}
$$

and

$$
(\bar{A})_{p}\left(\left[x_{\alpha}\right]_{p}\right) \stackrel{\alpha}{\rightarrow} \overline{(\bar{A})_{p}}\left(x_{p}\right)
$$

This implies

$$
\mathrm{R}_{p}(\lambda, \bar{A})\left[\left(\lambda I-\overline{(\bar{A})_{p}}\right)\left(\left[x_{\alpha}\right]_{p}\right)\right]=(\mathrm{R}(\lambda, \bar{A}))_{p}\left[\left(\lambda I-(\bar{A})_{p}\right)\left(\left[x_{\alpha}\right]_{p}\right)\right]=\left[x_{\alpha}\right]_{p} \longrightarrow x_{p}
$$

But

$$
\left(\lambda I-\overline{(\bar{A})_{p}}\right)\left(\left[x_{\alpha}\right]_{p}\right)=\left(\lambda I-(\bar{A})_{p}\right)\left(\left[x_{\alpha}\right]_{p}\right) \longrightarrow\left(\lambda I-\overline{(\bar{A})_{p}}\right)\left(x_{p}\right),
$$

which implies

$$
\mathrm{R}_{p}(\lambda, \bar{A})\left[\left(\lambda I-\overline{(\bar{A})_{p}}\right)\left(\left[x_{\alpha}\right]_{p}\right)\right] \rightarrow \mathrm{R}_{p}(\lambda, \bar{A})\left[\left(\lambda I-\overline{(\bar{A})_{p}}\right)\left(x_{p}\right)\right],
$$

by the continuity of the operator $\mathrm{R}_{p}(\lambda, \bar{A})$. By uniqueness of the limit,

$$
\mathrm{R}_{p}(\lambda, \bar{A})\left[\left(\lambda I-\overline{(\bar{A})_{p}}\right)\left(x_{p}\right)\right]=x_{p}
$$

This proves that $\mathrm{R}_{p}(\lambda, \bar{A})$ is a left inverse for $\left(\lambda I-\overline{(\bar{A})_{p}}\right)$. To prove that $\mathrm{R}_{p}(\lambda, \bar{A})$ is a right inverse fix an arbitrary $x_{p} \in \mathcal{X}_{p}$ and a net $\left\{\left[x_{\alpha}\right]_{p}\right\}$ in $\pi_{p}[\mathcal{D}]$ such that $\left[x_{\alpha}\right]_{p} \longrightarrow x_{p}$. Then,

$$
\left(\lambda I-\overline{(\bar{A})_{p}}\right) \mathrm{R}_{p}(\lambda, \bar{A})\left(\left[x_{\alpha}\right]_{p}\right)=\left(\lambda I-(\bar{A})_{p}\right)(\mathrm{R}(\lambda, \bar{A}))_{p}\left(\left[x_{\alpha}\right]_{p}\right)=\left[x_{\alpha}\right]_{p} \longrightarrow x_{p} .
$$

On the other hand, continuity of $\mathrm{R}_{p}(\lambda, \bar{A})$ and closedness of $\overline{(\bar{A})_{p}}$ imply that

$$
\left(\lambda I-\overline{(\bar{A})_{p}}\right) \mathrm{R}_{p}(\lambda, \bar{A})\left(\left[x_{\alpha}\right]_{p}\right) \longrightarrow\left(\lambda I-\overline{(\bar{A})_{p}}\right) \mathrm{R}_{p}(\lambda, \bar{A})\left(x_{p}\right) .
$$

As before, uniqueness of the limit establishes that $\mathrm{R}_{p}(\lambda, \bar{A})$ is a right inverse for $\lambda I-\overline{(\bar{A})_{p}}$. This proves $\mathrm{R}_{p}(\lambda, \bar{A})$ is a two-sided inverse for the operator $\lambda I-\overline{(\bar{A})_{p}}$ defined on Dom $\overline{(\bar{A})_{p}}$ and that $\mathrm{R}_{p}(\lambda, \bar{A})=\left(\lambda I-\overline{(\bar{A})_{p}}\right)^{-1}$. Since $\mathrm{R}_{p}(\lambda, \bar{A})$ is a continuous operator, the equality

$$
\mathrm{R}_{p}(\lambda, \bar{A})=\mathrm{R}\left(\lambda, \overline{(\bar{A})_{p}}\right)
$$

follows, as claimed. 
Hence, combining all of these results with the hypothesis

$$
|\operatorname{Re} \lambda|>\|\tilde{a d} A\|
$$

one finally concludes that

$$
\left\|\mathrm{R}_{p}(\lambda, \bar{A})^{k}\right\|_{p}^{1 / k} \leq\left\|\mathrm{R}_{p}(\lambda, \bar{A})\right\|_{p}=\left\|\mathrm{R}\left(\lambda, \overline{(\bar{A})_{p}}\right)\right\|_{p} \leq \frac{1}{|\operatorname{Re} \lambda|}<\frac{1}{\|\tilde{\mathrm{ad}} A\|}, \quad k \in \mathbb{N} .
$$

Noting that the sequence $\left\{\left\|\mathrm{R}_{p}(\lambda, \bar{A})^{k}\right\|^{1 / k}\right\}_{k \in \mathbb{N}}$ converges to

$$
\inf \left\{\left\|\mathrm{R}_{p}(\lambda, \bar{A})^{k}\right\|_{p}^{1 / k}: k \in \mathbb{N}\right\}=\nu\left(\mathrm{R}_{p}(\lambda, \bar{A})\right)
$$

one sees that

$$
\nu\left(\mathrm{R}_{p}(\lambda, \bar{A})\right)<\frac{1}{\|\tilde{\mathrm{ad}} A\|}
$$

and

$$
\nu\left(\mathrm{R}_{p}(\lambda, \bar{A})\right) \nu(\tilde{\operatorname{ad}} A)<\frac{1}{\|\tilde{\mathrm{ad}} A\|}\|\tilde{\mathrm{ad}} A\|=1 .
$$

This gives the desired result and shows that there exist $M>0$ and $0<r_{p}<1$ such that

$$
p\left(\mathrm{R}(\lambda, \bar{A})^{k}\left[(\operatorname{ad} A)^{k}(B) x\right]\right) \leq M r_{p}^{k} \rho_{p, 1}(x), \quad x \in \mathcal{D},
$$

for sufficiently large $k$. Therefore,

$$
\sum_{k=0}^{+\infty} p\left(\mathrm{R}(\lambda, \bar{A})^{k}(\operatorname{ad} A)^{k}(B) x\right)<\infty, \quad x \in \mathcal{D}
$$

which implies

$$
\lim _{k \rightarrow+\infty} p\left(\mathrm{R}(\lambda, \bar{A})^{k}(\operatorname{ad} A)^{k}(B) x\right)=0, \quad x \in \mathcal{D} .
$$

Since $p$ is arbitrary, $\mathrm{R}(\lambda, \bar{A})^{k}(\operatorname{ad} A)^{k}(B)(x) \longrightarrow 0$ in $\mathcal{X}$, for all $x \in \mathcal{D}$, and

$$
B \mathrm{R}(\lambda, \bar{A})(x)=\sum_{k=0}^{+\infty}(-1)^{k} \mathrm{R}(\lambda, \bar{A})^{k+1}\left[(\operatorname{ad} A)^{k}(B)\right](x), \quad x \in \operatorname{Ran}(\lambda I-A) .
$$

Now, the only step missing is to extend what was just proved to every element of $\mathcal{D}$. So let $y \in \mathcal{D}$. By the density hypothesis there exists a net $\left\{y_{\alpha}\right\}$ in $\operatorname{Ran}(\lambda I-A)$ such that $\rho_{p, 1}\left(y_{\alpha}-y\right) \stackrel{\alpha}{\rightarrow} 0$, for all $p \in \Gamma_{A}$. Fix $p^{\prime} \in \Gamma_{A}$. Using what was just proved one sees that, for all $\alpha$,

$$
\sum_{k=0}^{+\infty} p^{\prime}\left((-1)^{k} \mathrm{R}(\lambda, \bar{A})^{k+1}\left[(\operatorname{ad} A)^{k}(B)\left(y_{\alpha}-y\right)\right]\right)
$$




$$
\leq p_{1}^{\prime}\left(y_{\alpha}-y\right)\left\|\mathrm{R}_{p^{\prime}}(\lambda, \bar{A})\right\|_{p^{\prime}}\left[\sum_{k=0}^{+\infty} M r_{p^{\prime}}^{k}\right] .
$$

Since $p^{\prime}$ is arbitrary, taking limits on $\alpha$ on both sides gives, in particular, that

$$
\sum_{k=0}^{+\infty}(-1)^{k} \mathrm{R}(\lambda, \bar{A})^{k+1}\left[(\operatorname{ad} A)^{k}(B)\left(y_{\alpha}\right)\right] \longrightarrow \sum_{k=0}^{+\infty}(-1)^{k} \mathrm{R}(\lambda, \bar{A})^{k+1}\left[(\operatorname{ad} A)^{k}(B)(y)\right] .
$$

Hence, $\left\{B \mathrm{R}(\lambda, \bar{A})\left(y_{\alpha}\right)\right\}$ converges and, by closedness of $\bar{B}$ and $\tau$-continuity of $\mathrm{R}(\lambda, \bar{A})$,

$$
B \mathrm{R}(\lambda, \bar{A})\left(y_{\alpha}\right) \stackrel{\alpha}{\rightarrow} \bar{B} \mathrm{R}(\lambda, \bar{A})(y) .
$$

This implies

$$
\bar{B} \mathrm{R}(\lambda, \bar{A})(y)=\sum_{k=0}^{+\infty}(-1)^{k} \mathrm{R}(\lambda, \bar{A})^{k+1}\left[(\operatorname{ad} A)^{k}(B)\right](y), \quad y \in \mathcal{D},
$$

giving the desired result.

Theorem 2.4 below is a "locally convex version" of [74, Theorem 6.1, page 133], when $A$ is assumed to be a pregenerator of an equicontinuous group.

Theorem 2.4 ( $\mathrm{C}^{1}$-Continuity of One-Parameter Groups): Suppose also that all the basis operators $\left(B_{k}\right)_{1 \leq k \leq d}$ satisfy the (KIP) with respect to $\Gamma_{A}$. Then, $\bar{A}^{\tau_{1}}$ is the infinitesimal generator of (see Theorem 2.2 for the definition of this operator) $a$ $\Gamma_{A, 1}$-group of bounded type $t \longmapsto V_{1}\left(t, \bar{A}^{\tau_{1}}\right)$ on $\mathcal{D}_{1}$. Moreover, $V(t, \bar{A})$ leaves $\mathcal{D}_{1}$ invariant, for all $t \in \mathbb{R}$, and restricts there as the $\tau_{1}$-continuous linear operator $V_{1}\left(t, \bar{A}^{\tau_{1}}\right)$, for all $t \in \mathbb{R}$.

Every $B \in \mathcal{L}$ can be seen as a continuous linear operator from $\left(\mathcal{D}, \tau_{1}\right)$ to $(\mathcal{X}, \tau)$, so denote by $\tilde{B}^{(1)}: \mathcal{D}_{1} \longmapsto \mathcal{X}$ its unique continuous extension to all of $\mathcal{D}_{1}$. Then, for all closable $B \in \mathcal{L}$,

$$
\tilde{B}^{(1)} V_{1}\left(t, A_{1}\right)(x)=V(t, \bar{A})[\exp (-t a d A)(B)]^{\sim(1)}(x), \quad t \in \mathbb{R}, x \in \mathcal{D}_{1},
$$

where $\exp (-t$ ad $A)(B)$ is defined by the $|\cdot|_{1}$-convergent series

$$
\exp (-t a d A)(B):=\sum_{k=0}^{+\infty} \frac{(a d A)^{k}(B)}{k !}(-t)^{k} \in \mathcal{L} .
$$

Proof of Theorem 2.4: The first initiative of the proof is to obtain the hypotheses which are necessary to invoke the $(\Leftarrow)$ implication of $[8$, Theorem 4.2$]$, but for the complete 
Hausdorff locally convex space $\left(\mathcal{D}_{1}, \tau_{1}\right)$ (note that its topology is generated by a saturated family of seminorms). Let $\lambda \in \mathbb{C}$ satisfy $|\operatorname{Re} \lambda| \geq M_{A}:=\max \left\{\left|\operatorname{Re} \lambda_{(-, A)}\right|,\left|\operatorname{Re} \lambda_{(+, A)}\right|\right\}>$ 0 . The additional hypothesis that all the basis operators $\left(B_{k}\right)_{1 \leq k \leq d}$ satisfy the (KIP) with respect to $\Gamma_{A}$ will already be used in the following argument: $\bar{A}$ possesses the (KIP) with respect to $\Gamma_{A}$ and, since all the basis elements also possess the (KIP) with respect to $\Gamma_{A}$, the operator

$$
\bar{A}^{\tau_{1}}: \operatorname{Dom} \bar{A}^{\tau_{1}} \subseteq \mathcal{D}_{1} \longrightarrow \mathcal{D}_{1}
$$

is a $\tau_{1}$-densely defined $\tau_{1}$-closed linear operator on $\mathcal{D}_{1}$ which possesses the (KIP) with respect to $\Gamma_{A, 1}$, since $\bar{A}^{\tau_{1}} \subset \bar{A}$ : in fact, if $\rho_{p, 1} \in \Gamma_{A, 1}, N_{\rho_{p, 1}}:=\left\{x \in \mathcal{D}_{1}: \rho_{p, 1}(x)=0\right\}$ and $x \in \operatorname{Dom} \bar{A}^{\tau_{1}} \cap N_{\rho_{p, 1}}$, then $p(x)=0$, so $x \in N_{p}$, which implies $\bar{A}^{\tau_{1}} x=\bar{A} x \in N_{p}$; by the (KIP) of the basis elements, it follows that $B_{k}\left(\bar{A}^{\tau_{1}} x\right)=B_{k}(\bar{A} x) \in N_{p}$, for all $1 \leq k \leq d$, so $\bar{A}^{\tau_{1}} x \in N_{\rho_{p, 1}}$, proving $\bar{A}^{\tau_{1}}$ has the (KIP) with respect to $\Gamma_{A, 1}$.

Therefore, ${ }^{7}$

$$
\left(\bar{A}^{\tau_{1}}\right)_{\rho_{p, 1}}: \pi_{\rho_{p, 1}}\left[\operatorname{Dom} \bar{A}^{\tau_{1}}\right] \longrightarrow\left(\mathcal{D}_{1}\right)_{\rho_{p, 1}}
$$

is a (well-defined) densely defined linear operator, for all $\rho_{p, 1} \in \Gamma_{A, 1}$. (For the rest of the proof, the more economic notations $A_{1}:=\bar{A}^{\tau_{1}}$ and $A_{\rho_{p, 1}}:=\left(A_{1}\right)_{\rho_{p, 1}}=\left(\bar{A}^{\tau_{1}}\right)_{\rho_{p, 1}}$ are going to be employed, in order to facilitate the reading process) To see that it is $\|\cdot\|_{\rho_{p, 1}}$-closable, a strategy which is completely analogous to the one employed in the proof of Lemma 1.3.2 will be used. Note first that

$$
p\left((\operatorname{ad} A)\left(B_{k}\right) x\right) \leq\left|(\operatorname{ad} A)\left(B_{j}\right)\right|_{1} \rho_{p, 1}(x) \leq\|\tilde{a d} A\| \rho_{p, 1}(x),
$$

for all $p \in \Gamma_{A}, 1 \leq k \leq d$ and $x \in \mathcal{D}$, since $\left|B_{k}\right|_{1}=1$. Hence, since $A$ is $\Gamma_{A}$-conservative, one obtains for each $p \in \Gamma_{A}$ and $1 \leq k \leq d$ that

$$
\begin{gathered}
p\left(B_{k}(\mu I-A) x\right)=p\left((\mu I-A) B_{k}(x)+(\operatorname{ad} A)\left(B_{k}\right)(x)\right) \\
\geq p\left((\mu I-A) B_{k}(x)\right)-p\left((\operatorname{ad} A)\left(B_{k}\right) x\right) \\
\geq|\mu| p\left(B_{k}(x)\right)-\|\tilde{a} d A\| \rho_{p, 1}(x), \quad|\mu|>\|\tilde{a d} A\|, x \in \mathcal{D} .
\end{gathered}
$$

Taking the maximum over $0 \leq k \leq d$ produces

$$
\rho_{p, 1}((\mu I-A) x) \geq(|\mu|-\|\tilde{a d} A\|) \rho_{p, 1}(x), \quad|\mu|>\|\tilde{a d} A\|, x \in \mathcal{D} .^{8}
$$

Now, let $\left\{\left[x_{\alpha}\right]_{\rho_{p, 1}}\right\}$ be a net in $\pi_{p}\left[\operatorname{Dom} A_{1}\right]$ and $\left\{A_{\rho_{p, 1}}\left(\left[x_{\alpha}\right]_{\rho_{p, 1}}\right)\right\}=\left\{\left[A_{1}\left(x_{\alpha}\right)\right]_{\rho_{p, 1}}\right\}$ be a net in $\mathcal{D}_{1} / N_{\rho_{p, 1}}$, where

$$
\left[x_{\alpha}\right]_{\rho_{p, 1}} \stackrel{\alpha}{\rightarrow} 0
$$

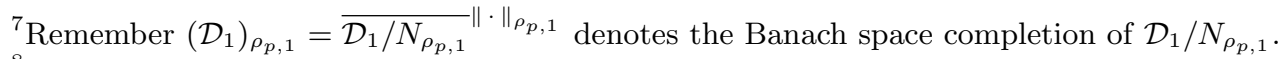

${ }^{8}$ Note that the case $k=0$ is just a consequence of $\Gamma_{A}$-conservativity of $A$.
} 
and

$$
\left[A_{1}\left(x_{\alpha}\right)\right]_{\rho_{p, 1}} \stackrel{\alpha}{\rightarrow} y \in\left(\mathcal{D}_{1}\right)_{\rho_{p, 1}},
$$

both convergences being in the $\|\cdot\|_{\rho_{p, 1}}$-topology. By the above inequality, one has for all $\mu>\|\tilde{a d} A\|$ and $\left[x^{\prime}\right]_{\rho_{p, 1}} \in \pi_{\rho_{p, 1}}[\mathcal{D}]$ that

$$
\begin{gathered}
\left\|\left(I-\frac{1}{\mu} A_{\rho_{p, 1}}\right)\left(\left[x_{\alpha}+\frac{1}{\mu} x^{\prime}\right]_{\rho_{p, 1}}\right)\right\|_{\rho_{p, 1}}=\left\|\left[\left(I-\frac{1}{\mu} A_{1}\right)\left(x_{\alpha}+\frac{1}{\mu} x^{\prime}\right)\right]_{\rho_{p, 1}}\right\|_{\rho_{p, 1}} \\
=\rho_{p, 1}\left(\left(I-\frac{1}{\mu} A_{1}\right)\left(x_{\alpha}+\frac{1}{\mu} x^{\prime}\right)\right) \geq \frac{\mu-\|\tilde{\mathrm{ad}} A\|}{\mu} \rho_{p, 1}\left(x_{\alpha}+\frac{1}{\mu} x^{\prime}\right) \\
=\frac{\mu-\|\tilde{\mathrm{ad}} A\|}{\mu}\left\|\left[x_{\alpha}+\frac{1}{\mu} x^{\prime}\right]_{\rho_{p, 1}}\right\| \|_{\rho_{p, 1}} .
\end{gathered}
$$

Hence,

$$
\begin{gathered}
\left\|\left[x_{\alpha}\right]_{\rho_{p, 1}}+\frac{1}{\mu}\left[x^{\prime}\right]_{\rho_{p, 1}}-\frac{1}{\mu} A_{\rho_{p, 1}}\left(\left[x_{\alpha}\right]_{\rho_{p, 1}}\right)-\frac{1}{\mu^{2}} A_{\rho_{p, 1}}\left(\left[x^{\prime}\right]_{\rho_{p, 1}}\right)\right\|_{\rho_{p, 1}} \\
\geq \frac{\mu-\|\tilde{\mathrm{ad}} A\|}{\mu}\left\|\left[x_{\alpha}\right]_{\rho_{p, 1}}+\frac{1}{\mu}\left[x^{\prime}\right]_{\rho_{p, 1}}\right\|_{\rho_{p, 1}} .
\end{gathered}
$$

Taking limits on $\alpha$ on both members and multiplying by $\mu$, one obtains

$$
\|\left[x^{\prime}\right]_{\rho_{p, 1}}-y-\frac{1}{\mu} A_{\rho_{p, 1}}\left(\left[x^{\prime}\right]_{\left.\rho_{p, 1}\right)}\left\|_{\rho_{p, 1}} \geq \frac{\mu-\|\tilde{\mathrm{ad}} A\|}{\mu}\right\|\left[x^{\prime}\right]_{\rho_{p, 1}} \|_{\rho_{p, 1}} .\right.
$$

Sending $\mu$ to $+\infty$ and using the density of $\pi_{\rho_{p, 1}}[\mathcal{D}]$ in $\left(\mathcal{D}_{1}\right)_{\rho_{p, 1}}$, it follows that $\|y\|_{\rho_{p, 1}}=0$. This shows $y=0$ and proves the $\|\cdot\|_{\rho_{p, 1}}$-closability of $A_{\rho_{p, 1}}$.

To prove the necessary resolvent bounds, substitute $B$ by $B_{j}$ on equation (2.3.1) of Theorem 2.3, $1 \leq j \leq d$, where $\left\{B_{j}\right\}_{1 \leq j \leq d}$ is the fixed basis of $\mathcal{L}$. This gives

$$
\overline{B_{j}} \mathrm{R}(\lambda, \bar{A})(x)=\sum_{k=0}^{+\infty}(-1)^{k} \mathrm{R}(\lambda, \bar{A})^{k+1}\left[(\operatorname{ad} A)^{k}\left(B_{j}\right)\right](x), \quad 1 \leq j \leq d, x \in \mathcal{D} .
$$

Now, since Theorem 2.3 implies $\mathrm{R}_{p}(\lambda, \bar{A})$ is the $\lambda$-resolvent of $\overline{(\bar{A})_{p}}$, which is the infinitesimal generator of a group of isometries,

$$
\frac{1}{\|\tilde{\mathrm{ad}} A\|}>\frac{1}{|\operatorname{Re} \lambda|} \geq\left\|\mathrm{R}_{p}(\lambda, \bar{A})\right\|_{p}
$$


Hence, $\left\|\mathrm{R}_{p}(\lambda, \bar{A})\right\|_{p}\|\tilde{a d} A\|<1$. This implies

$$
\begin{aligned}
& p\left(\overline{B_{j}} \mathrm{R}(\lambda, \bar{A}) x\right) \leq\left\|\mathrm{R}_{p}(\lambda, \bar{A})\right\|_{p}\left[\sum_{k=0}^{+\infty} p\left(\mathrm{R}(\lambda, \bar{A})^{k}\left[(\operatorname{ad} A)^{k}\left(B_{j}\right)\right] x\right)\right] \\
& \leq\left\|\mathrm{R}_{p}(\lambda, \bar{A})\right\|_{p}\left[\sum_{k=0}^{+\infty}\left\|\mathrm{R}_{p}(\lambda, \bar{A})\right\|_{p}^{k}\|\tilde{a d} A\|^{k}\left|B_{j}\right|_{1} \rho_{p, 1}(x)\right] \\
& =\frac{\left\|\mathrm{R}_{p}(\lambda, \bar{A})\right\|_{p}}{1-\left\|\mathrm{R}_{p}(\lambda, \bar{A})\right\|_{p}\|\tilde{\mathrm{ad}} A\|} \rho_{p, 1}(x)=\frac{1}{\frac{1}{\left\|\mathrm{R}_{p}(\lambda, \bar{A})\right\|_{p}}-\|\tilde{\mathrm{ad}} A\|} \rho_{p, 1}(x) \\
& \leq \frac{1}{|\operatorname{Re} \lambda|-\|\tilde{\operatorname{ad}} A\|} \rho_{p, 1}(x), \quad p \in \Gamma_{A}, 1 \leq j \leq d, x \in \mathcal{D},
\end{aligned}
$$

since $\left|B_{j}\right|_{1}=1$. Also,

$$
\begin{gathered}
p\left(\overline{B_{0}} \mathrm{R}(\lambda, \bar{A}) x\right)=p(\mathrm{R}(\lambda, \bar{A}) x) \leq\left\|\mathrm{R}_{p}(\lambda, \bar{A})\right\|_{p} p(x) \\
\leq \frac{\left\|\mathrm{R}_{p}(\lambda, \bar{A})\right\|_{p}}{1-\left\|\mathrm{R}_{p}(\lambda, \bar{A})\right\|_{p}\|\tilde{\operatorname{ad}} A\|} \rho_{p, 1}(x) \leq \frac{1}{|\operatorname{Re} \lambda|-\|\tilde{a d} A\|} \rho_{p, 1}(x), \quad p \in \Gamma_{A}, x \in \mathcal{D},
\end{gathered}
$$

because $0<1-\left\|\mathrm{R}_{p}(\lambda, \bar{A})\right\|_{p}\|\tilde{a d} A\|<1$. Therefore, taking the maximum over $0 \leq j \leq d$ and using the fact (proved in Theorem 2.2) that $\left.\mathrm{R}(\lambda, \bar{A})\right|_{\mathcal{D}_{1}}=\mathrm{R}\left(\lambda, A_{1}\right)$, a $\tau_{1}$-density argument provides

$$
\rho_{p, 1}\left(\mathrm{R}\left(\lambda, A_{1}\right) x\right) \leq \frac{1}{|\operatorname{Re} \lambda|-\|\tilde{\mathrm{ad}} A\|} \rho_{p, 1}(x), \quad p \in \Gamma_{A}, x \in \mathcal{D}_{1},
$$

showing that the operator $\left.\mathrm{R}(\lambda, \bar{A})\right|_{\mathcal{D}_{1}}$ possesses the (KIP) relatively to the family $\Gamma_{A, 1}$. This yields

$$
\left\|\left(\mathrm{R}\left(\lambda, A_{1}\right)\right)_{\rho_{p, 1}}\left([x]_{\rho_{p, 1}}\right)\right\|_{\rho_{p, 1}} \leq \frac{1}{|\operatorname{Re} \lambda|-\|\tilde{\mathrm{ad}} A\|}\left\|[x]_{\rho_{p, 1}}\right\|_{\rho_{p, 1}}, \quad p \in \Gamma_{A},[x]_{\rho_{p, 1}} \in \mathcal{D}_{1} / N_{\rho_{p, 1}} .
$$

Since $\mathcal{D}_{1} / N_{\rho_{p, 1}}$ is $\|\cdot\|_{\rho_{p, 1}}$-dense in $\left(\mathcal{D}_{1}\right)_{\rho_{p, 1}}$, the above estimate allows one to define by limits the unique continuous linear operator $\mathrm{R}_{\rho_{p, 1}}\left(\lambda, A_{1}\right)$ on $\left(\mathcal{D}_{1}\right)_{\rho_{p, 1}}$ which extends $\mathrm{R}\left(\lambda, A_{1}\right)$. Arguing in a similar manner of that done at the end of the first paragraph of Theorem 2.3 (exploring the closedness of the operator $\overline{A_{\rho_{p, 1}}}$ ), it follows that

$$
\mathrm{R}_{\rho_{p, 1}}\left(\lambda, A_{1}\right)=\mathrm{R}\left(\lambda, \overline{A_{\rho_{p, 1}}}\right) .
$$

Hence,

$$
\left\|\mathrm{R}\left(\lambda, \overline{A_{\rho_{p, 1}}}\right)\left(x_{\rho_{p, 1}}\right)\right\|_{\rho_{p, 1}} \leq \frac{1}{|\operatorname{Re} \lambda|-\|\tilde{\mathrm{ad}} A\|}\left\|x_{\rho_{p, 1}}\right\|_{\rho_{p, 1}}, \quad p \in \Gamma_{A}, x_{\rho_{p, 1}} \in\left(\mathcal{D}_{1}\right)_{\rho_{p, 1}} .
$$


In particular,

$$
\left\|\left[\mathrm{R}\left(\lambda, \overline{A_{\rho_{p, 1}}}\right)\right]^{n}\left(x_{\rho_{p, 1}}\right)\right\|_{\rho_{p, 1}} \leq \frac{1}{(|\lambda|-\|\tilde{\mathrm{ad}} A\|)^{n}}\left\|x_{\rho_{p, 1}}\right\|_{\rho_{p, 1}}, \quad p \in \Gamma_{A}, n \in \mathbb{N}, x_{\rho_{p, 1}} \in\left(\mathcal{D}_{1}\right)_{\rho_{p, 1}},
$$

for all $\lambda \in \mathbb{R}$ satisfying $|\lambda|>\|\tilde{a d} A\|$, which finishes the verification of the last hypothesis needed to apply Hille-Yosida-Phillips theorem for locally convex spaces ([8, Theorem 4.2]), ${ }^{9}$ so that $A_{1}$ is the generator of a $\Gamma_{A_{1}}$-group $t \longmapsto \mathrm{V}_{1}\left(t, A_{1}\right)$ on $\mathcal{D}_{1}$.

Fix $\lambda \in \mathbb{C}$ satisfying $\operatorname{Re} \lambda \geq M_{A}:=\max \left\{\left|\operatorname{Re} \lambda_{(-, A)}\right|,\left|\operatorname{Re} \lambda_{(+, A)}\right|\right\}>\|\tilde{a d} A\|$.

Since

$$
\mathrm{R}\left(\lambda, A_{1}\right)=\left.\mathrm{R}(\lambda, \bar{A})\right|_{\mathcal{D}_{1}}
$$

it follows for all $x \in \mathcal{D}_{1}$ that

$$
\int_{0}^{+\infty} e^{-\lambda s} V(s, \bar{A}) x d s=\mathrm{R}(\lambda, \bar{A}) x=\mathrm{R}\left(\lambda, A_{1}\right) x=\int_{0}^{+\infty} e^{-\lambda s} V_{1}\left(s, A_{1}\right) x d s,
$$

where the member on the left-hand side is a strongly $\tau$-convergent integral and the member on the right-hand side is a strongly $\tau_{1}$-convergent integral. Therefore, for fixed $x \in \mathcal{D}_{1}$ and $f \in \mathcal{X}^{\prime}$

$$
\int_{0}^{+\infty} e^{-\lambda s} f\left(V(s, \bar{A}) x-V_{1}\left(s, A_{1}\right) x\right) d s=0
$$

As the function

$$
f_{A}: s \longmapsto f\left(V(s, \bar{A}) x-V_{1}\left(s, A_{1}\right) x\right)
$$

is continuous on $[0,+\infty)$ and is such that $\left|f_{A}(s)\right| \leq C e^{a s}$, for all $s \in[0,+\infty)$ and some $C \geq 0, a \in \mathbb{R}$, it follows from the injectivity of the Laplace transform that $f(V(s, \bar{A}) x)=$ $f\left(V_{1}\left(s, A_{1}\right) x\right)$, for all $s \in[0,+\infty) .{ }^{10}$ By a corollary of Hahn-Banach's theorem, it follows that $\left.V(s, \bar{A})\right|_{\mathcal{D}_{1}}=V_{1}\left(s, A_{1}\right)$, for all $s \geq 0$. But if $s<0$, then

$$
V(s, \bar{A}) x=(V(-s, \bar{A}))^{-1} x=\left(V_{1}\left(-s, A_{1}\right)\right)^{-1} x=V_{1}\left(s, A_{1}\right) x, \quad x \in \mathcal{D}_{1},
$$

SO

$$
\left.V(s, \bar{A})\right|_{\mathcal{D}_{1}}=V_{1}\left(s, A_{1}\right),
$$

for all $s \in \mathbb{R}$, proving that $s \longmapsto V(s, \bar{A})$ restricts to a $\Gamma_{A, 1}$-group on $\mathcal{D}_{1}$.

\footnotetext{
${ }^{9}$ Actually, a straightforward adaptation to a group version of [8, Theorem 4.2] must be performed, by considering projective limits of strongly continuous groups, instead of semigroups - note that [8, Theorem $4.2]$ regards generation of semigroups.

${ }^{10}$ See, for example [51, Corollary 8.1, page 267].
} 
Before proceeding it is important to note that, for all $x \in \mathcal{D}$, the sequence

$$
\left\{\sum_{k=0}^{n} \frac{(\operatorname{ad} A)^{k}(B)}{k !}(-t)^{k}(x)\right\}_{n \in \mathbb{N}}
$$

in $\mathcal{D}$ converges to the element

$$
\sum_{k=0}^{+\infty} \frac{(\operatorname{ad} A)^{k}(B)}{k !}(-t)^{k}(x) \in \mathcal{D}
$$

- see [74, Theorem 3.2, page 64] - because the family $\{\mathcal{L} \ni C \longmapsto p(C x): p \in \Gamma\}$ of seminorms generates a Hausdorff topology on $\mathcal{L}$ and any two Hausdorff topologies on a finitedimensional vector space are equivalent.

The next step is to prove equality (2.4.1). Note, first, that $t \longmapsto \mathrm{V}_{1}\left(t, A_{1}\right)$ is of bounded type: if $w_{\rho_{p, 1}}$ denotes the type of the semigroup on $\left(\mathcal{D}_{1}\right)_{\rho_{p, 1}}$ induced (after being extended by limits $)^{11}$ by $[0,+\infty) \ni t \longmapsto V_{1}\left(t, A_{1}\right)$, then $w_{\rho_{p, 1}} \leq \|$ ad $A \|$, for all $\rho_{p, 1} \in \Gamma_{A, 1}$. This implies

$$
w:=\sup \left\{w_{\rho_{p, 1}}: \rho_{p, 1} \in \Gamma_{A, 1}\right\} \leq\|\tilde{a d} A\|,
$$

so $t \longmapsto V_{1}\left(t, A_{1}\right)$ is of bounded type, following the terminology of [8, page 170]. Fix, as before, $\lambda \in \mathbb{C}$ satisfying $\operatorname{Re} \lambda \geq M_{A}$. By what was just noted, [8, Theorem 3.3] is applicable and the relation

$$
\mathrm{R}\left(\lambda, A_{1}\right) x=\int_{0}^{+\infty} e^{-\lambda s} V_{1}\left(s, A_{1}\right) x d s
$$

is valid, for all $x \in \mathcal{D}_{1}$, where the integral is $\tau_{1}$-convergent. Also, $\left(\tau_{1} \times \tau\right)$-continuity of $\tilde{B}^{(1)}$ ensures that

$$
\tilde{B}^{(1)} \mathrm{R}\left(\lambda, A_{1}\right) x=\int_{0}^{+\infty} e^{-\lambda s} \tilde{B}^{(1)} V_{1}\left(s, A_{1}\right) x d s, \quad x \in \mathcal{D}_{1},
$$

the integral being $\tau$-convergent. The next step will be to prove that

$$
\begin{gathered}
\int_{0}^{+\infty} e^{-\lambda s} V(s, \bar{A})[\exp (-s \operatorname{ad} A)(B)]^{\sim(1)}(x) d s \\
=\sum_{n=0}^{+\infty}\left[\int_{0}^{+\infty} e^{-\lambda s} \frac{(-s)^{n}}{n !} V(s, \bar{A})(\operatorname{ad} A)^{n}(B)(x) d s\right], \quad x \in \mathcal{D}_{1} .
\end{gathered}
$$

\footnotetext{
${ }^{11}$ Note that the operators $V_{1}\left(t, A_{1}\right), t \geq 0$, possess the (KIP) with respect to $\Gamma_{A, 1}$, by the very definition of a $\Gamma_{A, 1}$-group.
} 
Fix $x \in \mathcal{D}, f \in \mathcal{X}^{\prime}$ and $p^{\prime} \in \Gamma_{A}$ such that $|f(x)| \leq M_{f} p^{\prime}(x)$, for all $x \in \mathcal{X}$. Now, for all $t \in \mathbb{R}$

$$
V(t, \bar{A})[\exp (-t \operatorname{ad} A)(B)]^{\sim(1)}(x)=\sum_{n=0}^{+\infty} \frac{(-t)^{n}}{n !} V(t, \bar{A})(\operatorname{ad} A)^{n}(B)(x),
$$

by $\tau$-continuity of the operator $V(t, \bar{A})$, and the integral

$$
\begin{aligned}
& \int_{0}^{+\infty}\left[\sum_{n=0}^{+\infty}\left|f\left(e^{-\lambda s} V(s, \bar{A}) \frac{(-s)^{n}}{n !}(\operatorname{ad} A)^{n}(B)(x)\right)\right| d s\right] \\
& \leq M_{f} \int_{0}^{+\infty} e^{-\lambda s}\left[\sum_{n=0}^{+\infty} \frac{s^{n}}{n !} p^{\prime}\left(V(s, \bar{A})(\operatorname{ad} A)^{n}(B)(x)\right) d s\right] \\
& =M_{f} \int_{0}^{+\infty} e^{-\lambda s}\left[\sum_{n=0}^{+\infty} \frac{s^{n}}{n !}\left\|(V(s, \bar{A}))_{p^{\prime}}\left[(\operatorname{ad} A)^{n}(B)(x)\right]_{p^{\prime}}\right\|_{p^{\prime}} d s\right] \\
& \leq M_{f} \int_{0}^{+\infty} e^{-\lambda s}\left[\sum_{n=0}^{+\infty} \frac{s^{n}}{n !}\left\|(V(s, \bar{A}))_{p^{\prime}}\right\|_{p^{\prime}} p^{\prime}\left((\operatorname{ad} A)^{n}(B)(x)\right) d s\right] \\
& \leq M_{f} \int_{0}^{+\infty} e^{-\lambda s}\left[\sum_{n=0}^{+\infty} \frac{s^{n}}{n !}\left|(\operatorname{ad} A)^{n}(B)\right|_{1} p_{1}^{\prime}(x) d s\right] \\
& \leq M_{f} \int_{0}^{+\infty} e^{-\lambda s}\left[\sum_{n=0}^{+\infty} \frac{(s\|\tilde{\mathrm{ad}} A\|)^{n}}{n !}|B|_{1} p_{1}^{\prime}(x) d s\right] \\
& =M_{f}|B|_{1} p_{1}^{\prime}(x) \int_{0}^{+\infty} e^{(-\lambda+\|\tilde{a} \mathrm{~d} A\|) s} d s<\infty
\end{aligned}
$$

converges, where it was used that the semigroup on $\mathcal{X} / N_{p^{\prime}}$ induced by $[0,+\infty) \ni t \longmapsto$ $V(t, \bar{A})$, being a contraction semigroup, satisfies

$$
\left\|(V(s, \bar{A}))_{p^{\prime}}\right\|_{p^{\prime}} \leq 1, \quad s \geq 0
$$

The convergence is ensured by the hypothesis $-\operatorname{Re} \lambda+\|\tilde{a d} A\|<0$. This justifies an application of Fubini's Theorem, from which it follows that

$$
\begin{aligned}
& \int_{0}^{+\infty} f\left(e^{-\lambda s} V(s, \bar{A})[\exp (-s \operatorname{ad} A)(B)]^{\sim(1)}(x) d s\right) \\
= & \int_{0}^{+\infty}\left[\sum_{n=0}^{+\infty} f\left(e^{-\lambda s} V(s, \bar{A}) \frac{(-s)^{n}}{n !}(\operatorname{ad} A)^{n}(B)(x)\right) d s\right]
\end{aligned}
$$




$$
=\sum_{n=0}^{+\infty}\left[\int_{0}^{+\infty} f\left(e^{-\lambda s} \frac{(-s)^{n}}{n !} V(s, \bar{A})(\operatorname{ad} A)^{n}(B)(x)\right) d s\right] .
$$

Finally, a Hahn-Banach's Theorem corollary gives the desired equality

$$
\begin{aligned}
& \int_{0}^{+\infty} e^{-\lambda s} V(s, \bar{A})[\exp (-s \operatorname{ad} A)(B)]^{\sim(1)}(x) d s \\
= & \sum_{n=0}^{+\infty}\left[\int_{0}^{+\infty} e^{-\lambda s} \frac{(-s)^{n}}{n !} V(s, \bar{A})(\operatorname{ad} A)^{n}(B)(x) d s\right] .
\end{aligned}
$$

On the other hand, a resolvent formula for closed linear operators on Banach spaces ${ }^{12}$ implies that

$$
\begin{gathered}
\frac{d^{n}}{d \lambda^{n}} \mathrm{R}_{p}(\lambda, \bar{A})\left([x]_{p}\right)=\frac{d^{n}}{d \lambda^{n}} \mathrm{R}\left(\lambda, \overline{(\bar{A})_{p}}\right)\left([x]_{p}\right)=(-1)^{n} n ! \mathrm{R}\left(\lambda, \overline{(\bar{A})_{p}}\right)^{n+1}\left([x]_{p}\right) \\
=(-1)^{n} n ! \mathrm{R}_{p}(\lambda, \bar{A})^{n+1}\left([x]_{p}\right), \quad p \in \Gamma_{A}, n \in \mathbb{N} .
\end{gathered}
$$

Thus

$$
\frac{d^{n}}{d \lambda^{n}} \mathrm{R}(\lambda, \bar{A})(x)=(-1)^{n} n ! \mathrm{R}(\lambda, \bar{A})^{n+1}(x),
$$

for all $n \in \mathbb{N}$, so

$$
\begin{gathered}
\sum_{n=0}^{+\infty}\left[\int_{0}^{+\infty} e^{-\lambda s} \frac{(-s)^{n}}{n !} V(s, \bar{A})(\operatorname{ad} A)^{n}(B)(x) d s\right] \\
=\sum_{n=0}^{+\infty} \frac{1}{n !}\left[\int_{0}^{+\infty} \frac{d^{n} e^{-\lambda s}}{d \lambda^{n}} V(s, \bar{A})(\operatorname{ad} A)^{n}(B)(x) d s\right] \\
=\sum_{n=0}^{+\infty} \frac{1}{n !}\left[\frac{d^{n}}{d \lambda^{n}} \int_{0}^{+\infty} e^{-\lambda s} V(s, \bar{A})(\operatorname{ad} A)^{n}(B)(x) d s\right]=\sum_{n=0}^{+\infty} \frac{1}{n !}\left[\frac{d^{n}}{d \lambda^{n}} \mathrm{R}(\lambda, \bar{A})(\operatorname{ad} A)^{n}(B)(x)\right] \\
=\sum_{n=0}^{+\infty}(-1)^{n} \mathrm{R}(\lambda, \bar{A})^{n+1}(\operatorname{ad} A)^{n}(B)(x)=\bar{B} \mathrm{R}(\lambda, \bar{A})(x)=\bar{B} \mathrm{R}\left(\lambda, A_{1}\right)(x),
\end{gathered}
$$

by formula (2.3.1) and $\mathrm{R}\left(\lambda, A_{1}\right)=\left.\mathrm{R}(\lambda, \bar{A})\right|_{\mathcal{D}_{1}}$. Since $\tilde{B}^{(1)} \subset \bar{B}$, the above equality together with equation (2.4.2) gives

$$
\int_{0}^{+\infty} e^{-\lambda s} V(s, \bar{A})[\exp (-s \operatorname{ad} A)(B)]^{\sim(1)}(x) d s=\int_{0}^{+\infty} e^{-\lambda s} \tilde{B}^{(1)} V_{1}\left(s, A_{1}\right) x d s .
$$

\footnotetext{
${ }^{12}$ To be more precise, let $T: \operatorname{Dom} T \subseteq \mathcal{Y} \longrightarrow \mathcal{Y}$ be a (not necessarily densely defined) closed linear operator on a Banach space $\mathcal{Y}$. If $\lambda$ is in the resolvent set of $T$, then

$$
\frac{d^{n}}{d \lambda^{n}} \mathrm{R}(\lambda, T)=(-1)^{n} n ! \mathrm{R}(\lambda, T)^{n+1}, \quad n \in \mathbb{N}
$$

- see [50, Proposition 1.3 (ii), page 240], for example.
} 
Using a corollary of Hahn-Banach's Theorem and injectivity of the Laplace transform, as before, formula (2.4.1) is proved for $t \geq 0$ and $x \in \mathcal{D}$.

Repeating the arguments of this last paragraph with $-A_{1}$, instead of $A_{1}$, and using the identity $V_{1}\left(t,-A_{1}\right)=V\left(-t, A_{1}\right)$, for all $t \geq 0$, gives the equality

$$
\tilde{B}^{(1)} V_{1}\left(t, A_{1}\right)(x)=V(t, \bar{A})[\exp (-t \text { ad } A)(B)]^{\sim(1)}(x), \quad t \leq 0, x \in \mathcal{D} .
$$

Now, the only thing missing is to extend formula (2.4.1) to all of $\mathcal{D}_{1}$, but this is easily accomplished by a $\tau_{1}$-density argument.

With the aid of the four theorems above one can finally prove the theorem which justifies the title of this section. Its proof follows the lines of [74, Theorem 7.4, page 161], establishing a similar result in the more general setting of complete Hausdorff locally convex spaces:

Theorem 2.5 (Construction of the Group Invariant $\mathbf{C}^{\infty}$ Domain): Let $(\mathcal{X}, \tau)$ be a complete Hausdorff locally convex space, $\mathcal{D}$ a dense subspace of $\mathcal{X}$ and $\mathcal{L} \subseteq \operatorname{End}(\mathcal{D})$ a finite dimensional real Lie algebra of linear operators. Fix an ordered basis $\left(B_{k}\right)_{1 \leq k \leq d}$ of $\mathcal{L}$ and define a basis-dependent norm on $\mathcal{L}$ by

$$
\left|\sum_{1 \leq k \leq d} b_{k} B_{k}\right|_{1}:=\sum_{1 \leq k \leq d}\left|b_{k}\right| .
$$

Suppose $\mathcal{L}$ is generated, as a Lie algebra, by a finite set $\mathcal{S}$ of infinitesimal pregenerators of equicontinuous groups and denote by $t \longmapsto V(t, \bar{A})$ the respective one-parameter group generated by $\bar{A}$, for all $A \in \mathcal{S}$. For each $A \in \mathcal{S}$ choose a fundamental system of seminorms $\Gamma_{A}$ for $\mathcal{X}$ with respect to which the operator $\bar{A}$ has the $(K I P)$, is $\Gamma_{A}$-conservative and $V(\cdot, \bar{A})$ is $\Gamma_{A}$-isometrically equicontinuous. Define $\Gamma_{A, 1}:=\left\{\rho_{p, 1}: p \in \Gamma_{A}\right\}$. Now, assume that the following hypotheses are verified:

1. the basis $\left(B_{k}\right)_{1 \leq k \leq d}$ is formed by closable elements having the (KIP) with respect to

$$
\Gamma_{\mathcal{S}}:=\bigcup_{A \in \mathcal{S}} \Gamma_{A}
$$

2. for each $A \in \mathcal{S}$ there exist two complex numbers $\lambda_{(-, A)}, \lambda_{(+, A)}$ satisfying $\operatorname{Re} \lambda_{(-, A)}<$ $-\|\tilde{a d} A\|$ and $\operatorname{Re} \lambda_{(+, A)}>\|\tilde{a d} A\|$, where $\|\tilde{a d} A\|$ denotes the usual operator norm of the extension ad $A: \mathcal{L}_{\mathbb{C}} \longrightarrow \mathcal{L}_{\mathbb{C}}$ such that, for all $\lambda \in \mathbb{C}$ satisfying

$$
|\operatorname{Re} \lambda| \geq M_{A}:=\max \left\{\left|\operatorname{Re} \lambda_{(-, A)}\right|,\left|\operatorname{Re} \lambda_{(+, A)}\right|\right\},
$$

the subspace Ran $(\lambda I-A)$ is dense in $\mathcal{D}$ with respect to the topology $\tau_{1}$ induced by the family $\Gamma_{A, 1}$. 
Define $\mathcal{D}_{\mathcal{S}}$ as the smallest subspace of $\mathcal{X}$ (with respect to set inclusion) that contains $\mathcal{D}$ and is left invariant by the operators $\{V(t, \bar{A}): A \in \mathcal{S}, t \in \mathbb{R}\}$ - such subspace is welldefined since, by Theorem 2.4, $\mathcal{D}_{1}$ is left invariant by $V(t, \bar{A})$, for all $t \in \mathbb{R}$ and $A \in \mathcal{S}$; also, $\mathcal{D}_{\mathcal{S}} \subseteq \mathcal{D}_{1}$. Then:

1. $\mathcal{D}_{\mathcal{S}}$ is a $C^{\infty}$ domain for $\overline{\mathcal{S}}:=\{\bar{A}: A \in \mathcal{S}\}$, so $\mathcal{D}_{\mathcal{S}} \subseteq \mathcal{X}_{\infty}(\overline{\mathcal{S}})$ - for the definition of $\mathcal{X}_{\infty}(\overline{\mathcal{S}})$, see the beginning of this section.

2. The estimates

$$
\rho_{p, n}(V(t, \bar{A}) x) \leq \exp (n\|a d A\||t|) \rho_{p, n}(x)
$$

hold for every $A \in \mathcal{S}, p \in \Gamma_{A}, n \in \mathbb{N}, t \in \mathbb{R}$ and $x \in \mathcal{D}_{\mathcal{S}}$, where $\rho_{p, n}$ was defined at the beginning of the section. Define $\mathcal{D}_{n}$ as the $\tau_{n}$-closure of $\mathcal{D}$ inside the complete Hausdorff locally convex space $\left(\mathcal{X}_{n}, \tau_{n}\right)$, for each $n \geq 1$. Then, $\mathcal{D}_{n}$ is left invariant by the operators $\{V(t, \bar{A}): A \in \mathcal{S}, t \in \mathbb{R}\}$ so, in particular, the one-parameter groups $t \longmapsto V(t, \bar{A})$ restrict to exponentially equicontinuous groups on $\mathcal{D}_{n}$ of type $\left\{w_{p}\right\}_{p \in \Gamma_{A}}$, where $w_{p} \leq n \|$ ad $A \|$, for each $n \geq 1$ and $p \in \Gamma_{A}$. By formula (2.5.1), they are all locally equicontinuous one-parameter groups.

3. The complete Hausdorff locally convex space

$$
\mathcal{D}_{\infty}:=\bigcap_{n=1}^{+\infty} \mathcal{D}_{n},
$$

equipped with the $\tau_{\infty}$-topology generated by the family

$$
\Gamma_{A, \infty}:=\bigcup_{n=0}^{+\infty} \Gamma_{A, n}
$$

of seminorms, for some fixed $A \in \mathcal{S}$ (note that this topology does not depend on the choice of $A \in \mathcal{S})$, is left invariant by the operators $\{V(t, \bar{A}): A \in \mathcal{S}, t \in \mathbb{R}\}$, and the restriction of the one-parameter groups $t \longmapsto V(t, \bar{A})$ to $\mathcal{D}_{\infty}$ are strongly continuous one-parameter groups of $\tau_{\infty}$-continuous operators. By (2.5.1), these restricted groups are locally equicontinuous, so each $t \longmapsto V(t, \bar{A})$ is a $\Gamma_{A, \infty}$-group on $\mathcal{D}_{\infty}$, where $A \in \mathcal{S}$. The same conclusions are true for the space ${\overline{\mathcal{D}_{\mathcal{S}}}}^{\infty}$, defined as the closure of $\mathcal{D}_{\mathcal{S}}$ inside the complete Hausdorff locally convex space $\left(\mathcal{X}_{\infty}(\overline{\mathcal{S}}), \tau_{\infty}\right)$. Even more, $\overline{\mathcal{D}}_{\mathcal{S}}^{\infty}$ is a $C^{\infty}$-domain for $\overline{\mathcal{S}}$.

4. The Lie algebra $\mathcal{L}_{\infty}:=\left\{\left.\bar{B}\right|_{\overline{\mathcal{D}}_{\mathcal{S}}}: B \in \mathcal{L}\right\}$ is isomorphic to $\mathcal{L}$.

Proof of Theorem 2.5: Proof of (1): To see that $\mathcal{D}_{\mathcal{S}}$ is a $\mathrm{C}^{\infty}$-domain, define inductively a non-decreasing sequence of subspaces by

$$
D_{\mathcal{S}}^{0}:=\mathcal{D}, \quad \mathcal{D}_{\mathcal{S}}^{n+1}:=\operatorname{span}_{\mathbb{C}}\left\{V(t, \bar{A}) x, t \in \mathbb{R}, A \in \mathcal{S}, x \in \mathcal{D}_{\mathcal{S}}^{n}\right\}, \text { for } n \geq 0,
$$


and

$$
\mathcal{D}_{\mathcal{S}}^{\infty}:=\bigcup_{n=0}^{+\infty} \mathcal{D}_{\mathcal{S}}^{n} .
$$

Since each $V(t, \bar{A})$ sends $\mathcal{D}_{\mathcal{S}}^{n}$ into $\mathcal{D}_{\mathcal{S}}^{n+1}$, for all $n \in \mathbb{N}$, it follows that each $V(t, \bar{A})$ sends $\mathcal{D}_{\mathcal{S}}^{\infty}$ into itself. Besides that, $\mathcal{D} \subseteq \mathcal{D}_{\mathcal{S}}^{\infty}$, so $\mathcal{D}_{\mathcal{S}} \subseteq \mathcal{D}_{\mathcal{S}}^{\infty}$. Now, $\mathcal{D} \subseteq \mathcal{D}_{\mathcal{S}}$ implies

$$
\left[\sum_{j=1}^{l} c_{j} V\left(t_{j}, \overline{A_{j}}\right) x_{j}\right] \in \mathcal{D}_{\mathcal{S}},
$$

for every $l \in \mathbb{N}, c_{j} \in \mathbb{C}, t_{j} \in \mathbb{R}, A_{j} \in \mathcal{S}$ and $x_{j} \in \mathcal{D}$, proving that $\mathcal{D}_{\mathcal{S}}^{1} \subseteq \mathcal{D}_{\mathcal{S}}$. Applying this argument inductively one sees that $\mathcal{D}_{\mathcal{S}}^{n} \subseteq \mathcal{D}_{\mathcal{S}}$, for all $n \in \mathbb{N}$, proving that $\mathcal{D}_{\mathcal{S}}^{\infty} \subseteq \mathcal{D}_{\mathcal{S}}$. Hence, $\mathcal{D}_{\mathcal{S}}=\mathcal{D}_{\mathcal{S}}^{\infty}$.

By hypothesis, $\mathcal{D}_{\mathcal{S}}^{0}=\mathcal{D}$ is a $\mathrm{C}^{\infty}$ domain for $\overline{\mathcal{S}}$. Suppose, for some $n \geq 0$, that $\mathcal{D}_{\mathcal{S}}^{n}$ is a $\mathrm{C}^{\infty}$ domain for $\overline{\mathcal{S}}$. Since $\mathcal{D}_{\mathcal{S}} \subseteq \mathcal{D}_{1}$, equation (2.4.1), proved in Theorem 2.4, shows

$$
\tilde{B}^{(1)} V_{1}\left(t, A_{1}\right)(x)=V(t, \bar{A})[\exp (-t \operatorname{ad} A)(B)]^{\sim(1)}(x),
$$

for all closable $B \in \mathcal{L}, A \in \mathcal{S}, t \in \mathbb{R}$ and $x \in \mathcal{D}_{\mathcal{S}}$. Let $y=V(t, \bar{A}) x$ be an element in the spanning set of $\mathcal{D}_{\mathcal{S}}^{n+1}$, so that $t \in \mathbb{R}, A \in \mathcal{S}$ and $x \in \mathcal{D}_{\mathcal{S}}^{n}$. Then, since $\tilde{B}^{(1)}=\left.\bar{B}\right|_{\mathcal{D}_{1}}$ and $V_{1}\left(t, A_{1}\right)=\left.V(t, \bar{A})\right|_{\mathcal{D}_{1}}$, for all $t \in \mathbb{R}$,

$$
\bar{B}(y)=\bar{B} V(t, \bar{A})(x)=\tilde{B}^{(1)} V_{1}\left(t, A_{1}\right)(x)=V(t, \bar{A})[\exp (-t \operatorname{ad} A)(B)]^{\sim(1)}(x) .
$$

To see that $[\exp (-t \text { ad } A)(B)]^{\sim(1)}(x) \in \mathcal{D}_{\mathcal{S}}^{n}$, note that every $C \in \mathcal{L}$, with $C=\sum_{k=1}^{d} c_{k} B_{k}$, satisfies

$$
\tilde{C}^{(1)} \subset \sum_{k=1}^{d} c_{k} \overline{B_{k}}
$$

Indeed, if $z \in \mathcal{D}_{1}$ there exists a net $\left\{z_{\alpha}\right\}$ in $\mathcal{D}$ which converges to $z$ with respect to the $\tau_{1}$-topology, so $C\left(z_{\alpha}\right) \stackrel{\alpha}{\rightarrow} \tilde{C}^{(1)}(z)$ in the $\tau$-topology. But, by the definition of $\tau_{1}$, $B_{k}\left(z_{\alpha}\right) \stackrel{\alpha}{\rightarrow} \overline{B_{k}}(z)$, for all $1 \leq k \leq d$. Consequently,

$$
C\left(z_{\alpha}\right)=\sum_{k=1}^{d} c_{k} B_{k}\left(z_{\alpha}\right) \stackrel{\alpha}{\rightarrow} \sum_{k=1}^{d} c_{k} \overline{B_{k}}(z),
$$

proving that $\tilde{C}^{(1)}=\left.\sum_{k=1}^{d} c_{k} \overline{B_{k}}\right|_{\mathcal{D}_{1}}$, as desired. Applying this reasoning to the operator $C=\exp (-t$ ad $A)(B) \in \mathcal{L}$ and taking into account the hypothesis of $\mathcal{D}_{\mathcal{S}}^{n}$ being a $\mathrm{C}^{\infty}$ domain for $\overline{\mathcal{S}}$, the conclusion $[\exp (-t \text { ad } A)(B)]^{\sim(1)}(x) \in \mathcal{D}_{\mathcal{S}}^{n}$ follows. Hence, $\bar{B}(y) \in \mathcal{D}_{\mathcal{S}}^{n+1}$ which, by linearity of $\bar{B}$, proves that

$$
\bar{B}\left[\mathcal{D}_{\mathcal{S}}^{n+1}\right] \subseteq \mathcal{D}_{\mathcal{S}}^{n+1}
$$


Since $B$ was an arbitrary closable operator of $\mathcal{L}$, this establishes that each $\mathcal{D}_{\mathcal{S}}^{n}$ is a $\mathrm{C}^{\infty}$ domain for $\overline{\mathcal{S}}$. Therefore, $\mathcal{D}_{\mathcal{S}}$, being a union of $\mathrm{C}^{\infty}$ domains, is itself a $\mathrm{C}^{\infty}$ domain. Since $\mathcal{X}_{\infty}(\overline{\mathcal{S}})$ is the largest $\mathrm{C}^{\infty}$ domain for $\overline{\mathcal{S}}$, the inclusion

$$
\mathcal{D}_{\mathcal{S}} \subseteq \mathcal{X}_{\infty}(\overline{\mathcal{S}})
$$

must hold, finishing the proof of (1).

Proofs of (2) and (3): To prove

$$
\rho_{p, n}(V(t, \bar{A}) x) \leq \exp (n\|\operatorname{ad} A\||t|) \rho_{p, n}(x)
$$

holds for every $A \in \mathcal{S}, p \in \Gamma_{A}, n \in \mathbb{N}, t \in \mathbb{R}$ and $x \in \mathcal{D}_{\mathcal{S}}$, the idea is to proceed by induction. Note that the evaluation of the seminorms $\rho_{p, n}$ is legitimate, for all $n \in \mathbb{N}$, because $\mathcal{D}_{\mathcal{S}}$ is contained in the complete Hausdorff vector space

$$
\bigcap_{n=1}^{+\infty} \mathcal{X}_{n}=: \mathcal{X}_{\infty}=\mathcal{X}_{\infty}(\overline{\mathcal{S}})
$$

introduced at the beginning of this section. The case $n=0$ is immediate since, for each $A \in \mathcal{S}, t \longmapsto V(t, \bar{A})$ is a $\Gamma_{A}$-isometrically equicontinuous one-parameter group. Suppose that

$$
\rho_{p, n}(V(t, \bar{A}) x) \leq \exp (n\|\operatorname{ad} A\||t|) \rho_{p, n}(x)
$$

for a fixed $n \in \mathbb{N}$ and all $A \in \mathcal{S}, p \in \Gamma_{A}, x \in \mathcal{D}_{\mathcal{S}}$ and $t \in \mathbb{R}$. First, note that for a fixed $C \in \mathcal{L}$, with $C=\sum_{k=1}^{d} c_{k} B_{k}$,

$$
\rho_{p, n}\left(\tilde{C}^{(1)}(x)\right) \leq|C|_{1} \rho_{p, n+1}(x),
$$

for all $x \in \mathcal{D}: \rho_{p, n}\left(\tilde{C}^{(1)}(x)\right)=\rho_{p, n}(C(x))=p\left(B^{\mathrm{u}}(C(x))\right)$, where $B^{\mathrm{u}}$ is a certain monomial of size $n$ in the elements $\left(B_{k}\right)_{1 \leq k \leq d}$, by the definition of $\rho_{p, n}$. Then,

$$
\rho_{p, n}\left(\tilde{C}^{(1)}(x)\right)=p\left(B^{\mathrm{u}}\left(\sum_{k=1}^{d} c_{k} B_{k}\right)(x)\right) \leq\left(\sum_{k=1}^{d}\left|b_{k}\right|\right) \rho_{p, n+1}(x)=|C|_{1} \rho_{p, n+1}(x),
$$

establishing the desired inequality. Hence, for $A \in \mathcal{S}, p \in \Gamma_{A}, x \in \mathcal{D}, t \in \mathbb{R}$ and $1 \leq k \leq d$,

$$
\begin{aligned}
\rho_{p, n}\left(\overline{B_{k}} V(t, \bar{A}) x\right)=\rho_{p, n}\left(\tilde{B}_{k} V(t, \bar{A}) x\right)=\rho_{p, n}\left(V(t, \bar{A})\left[\exp (-t \operatorname{ad} A)\left(B_{k}\right)\right]^{\sim(1)} x\right) \\
\leq \exp (n\|\operatorname{ad} A\||t|) \rho_{p, n}\left(\left[\exp (-t \operatorname{ad} A)\left(B_{k}\right)\right]^{\sim(1)} x\right) \\
\leq \exp (n\|\operatorname{ad} A\||t|)\left|\exp (-t \operatorname{ad} A)\left(B_{k}\right)\right|_{1} \rho_{p, n+1}(x) \\
\leq \exp (n\|\operatorname{ad} A\||t|)\|\exp (-t \operatorname{ad} A)\|\left|B_{k}\right|_{1} \rho_{p, n+1}(x)
\end{aligned}
$$




$$
\leq \exp (n\|\operatorname{ad} A\||t|) \exp (|t|\|\operatorname{ad} A\|) \rho_{p, n+1}(x)=\exp ((n+1)\|\operatorname{ad} A\||t|) \rho_{p, n+1}(x) .
$$

Upon taking the maximum over $0 \leq k \leq d$ - the estimate for $k=0$ is trivial - the inequality

$$
(2.5 .1)^{\prime} \quad \rho_{p, n+1}(V(t, \bar{A}) x) \leq \exp ((n+1)\|\operatorname{ad} A\||t|) \rho_{p, n+1}(x)
$$

follows. Now, note that the inequality just obtained implies that $V(t, \bar{A})$ leaves $\mathcal{D}_{n+1}$ invariant: indeed, fix $x \in \mathcal{D}_{n+1}$ and let $\left\{x_{\alpha}\right\}_{\alpha \in \mathcal{A}}$ be a net in $\mathcal{D}$ which $\tau_{n+1}$-converges to $x \in \mathcal{D}_{n+1}$. Then, by the inequality above,

$$
\rho_{p, n+1}\left(V(t, \bar{A})\left(x_{\alpha}-x_{\alpha^{\prime}}\right)\right) \leq \exp ((n+1)\|\operatorname{ad} A\||t|) \rho_{p, n+1}\left(x_{\alpha}-x_{\alpha^{\prime}}\right),
$$

for all $\alpha, \alpha^{\prime} \in \mathcal{A}$. This implies $\left\{V(t, \bar{A})\left(x_{\alpha}\right)\right\}_{\alpha \in \mathcal{A}}$ is a $\tau_{n+1}$-Cauchy net and, since $\mathcal{D}_{n+1}$ is complete, it follows this net is $\tau_{n+1}$-convergent to an element in $\mathcal{D}_{n+1}$. Since the $\tau_{n+1}$-topology is stronger that the $\tau$-topology, this element must be $V(t, \bar{A}) x$. Therefore, $V(t, \bar{A}) x \in \mathcal{D}_{n+1}$, for all $A \in \mathcal{S}$. By the definition of $\mathcal{D}_{\mathcal{S}}$,

$$
\mathcal{D}_{\mathcal{S}} \subseteq \mathcal{D}_{n+1}
$$

The argument just exposed proves (2.5.1)' not only for $x \in \mathcal{D}$, but establishes it for all $x \in \mathcal{D}_{n+1}$. In particular, it is valid for all $x \in \mathcal{D}_{\mathcal{S}}$, finishing the induction proof. Hence, (2.5.1) is true and, by the proof just developed, it follows that $V(t, \bar{A})$ leaves $\mathcal{D}_{n}$ invariant, for all $A \in \mathcal{S}, t \in \mathbb{R}, n \geq 1$. Moreover, (2.5.1) and a $\tau_{n}$-density argument of $\mathcal{D}_{\mathcal{S}}$ in $\mathcal{D}_{n}$ proves (just as it was already noted during the induction proof) that each $V(t, \bar{A})$ is a $\tau_{n}$-continuous operator on $\mathcal{D}_{n}$ and that (2.5.1) extends to $x \in \mathcal{D}_{n}$, for each fixed $n \geq 1$. This is a fact which will be used in the next argument, to prove that the restrictions of the one-parameter groups $t \longmapsto V(t, \bar{A})$ to $\mathcal{D}_{n}$ are strongly continuous with respect to the topology $\tau_{n}$, for every $n \geq 1$.

The estimates

$$
\begin{gathered}
\sum_{k=0}^{+\infty} \rho_{p, n}\left(\frac{(\operatorname{ad} A)^{k}\left(B_{j}\right)}{k !}(-t)^{k}(x)-B_{j}(x)\right) \leq \sum_{k=1}^{+\infty} \frac{\left|(\operatorname{ad})^{k}\left(B_{j}\right)\right|_{1}}{k !}|t|^{k} \rho_{p, n+1}(x) \\
\leq \rho_{p, n+1}(x) \sum_{k=1}^{+\infty} \frac{\|\operatorname{ad} A\|^{k}}{k !}|t|^{k}, \quad t \in \mathbb{R}, p \in \Gamma_{A}, x \in \mathcal{D}
\end{gathered}
$$

show each $\exp (-t$ ad $A)\left(B_{j}\right)(x) \tau_{n}$-converges to $B_{j}(x)$, when $t \longrightarrow 0$, for all $n \in \mathbb{N}$ and $1 \leq j \leq d$. Hence, using formula (2.5.1), one sees that $V(t, \bar{A}) \exp (-t$ ad $A)\left(B_{j}\right)(x) \tau_{n^{-}}$ converges to $B_{j}(x)$, for all $A \in \mathcal{S}, n \in \mathbb{N}$ and $1 \leq j \leq d$. Therefore, an application of formula (2.4.1) shows that if $t \longmapsto V(t, \bar{A}) x$ is $\tau_{n}$-continuous for some fixed $n \in \mathbb{N}$ and all $x \in \mathcal{D}$, then $t \longmapsto B_{j} V(t, \bar{A}) x$ is also $\tau_{n}$-continuous for all $x \in \mathcal{D}$, where $A \in \mathcal{S}$ and $1 \leq j \leq d$. But $t \longmapsto V(t, \bar{A}) x$ is $\tau_{0}$-continuous, for every $A \in \mathcal{S}$ and $x \in \mathcal{D}$, so an 
inductive argument on $n$, followed by a $\tau_{n}$-density argument of $\mathcal{D}$ in $\mathcal{D}_{n}$ combined with an $\epsilon / 3$-argument, shows that the group $t \longmapsto V(t, \bar{A})$ restricts to an exponentially equicontinuous group on $\mathcal{D}_{n}$ with type $w_{p} \leq n \|$ ad $A \|$, where $p \in \Gamma_{A}$ and $n \geq 1$, which ends the proof of (2). Also, this proves that the restriction of the groups $t \longmapsto V(t, \bar{A})$ to the complete Hausdorff locally convex space $\mathcal{D}_{\infty}$, equipped with the $\tau_{\infty}$-topology, are strongly continuous one-parameter groups of $\tau_{\infty}$-continuous operators. Consequently, for each fixed $A \in \mathcal{S}, t \longmapsto V(t, \bar{A})$ restricts to a $\Gamma_{A, \infty}$-group on $\mathcal{D}_{\infty}$, since local equicontinuity follows at once from estimates (2.5.1), after extending them to all $x \in \mathcal{D}_{n}$, for each fixed $n \geq 1$.

By the group invariance just mentioned, together with completeness of $\left(\mathcal{D}_{\infty}, \tau_{\infty}\right)$, the chain of inclusions

$$
\mathcal{D}_{\mathcal{S}} \subseteq{\overline{\mathcal{D}_{\mathcal{S}}}}^{\infty} \subseteq \mathcal{D}_{\infty} \subseteq \mathcal{X}_{\infty}(\overline{\mathcal{S}})
$$

is true. But even more is true: estimates (2.5.1) show, together with a $\tau_{\infty}$-density argument, that the $\tau_{\infty}$-continuous operators $V(t, \bar{A}), A \in \mathcal{S}, t \in \mathbb{R}$, leave $\overline{\mathcal{D}}_{\mathcal{S}}{ }^{\infty}$ invariant. This observation, combined with the strong continuity claim of the previous paragraph and the inclusion ${\overline{\mathcal{D}_{\mathcal{S}}}}^{\infty} \subseteq \mathcal{D}_{\infty}$, above, shows that each $t \longmapsto V(t, \bar{A})$ is a $\Gamma_{A, \infty}$-group on ${\overline{\mathcal{D}_{\mathcal{S}}}}^{\infty}$, where $A$ belongs to $\mathcal{S}$. The fact that $\overline{\mathcal{D}}_{\mathcal{S}}^{\infty}$ is a $\mathrm{C}^{\infty}$ domain for $\overline{\mathcal{S}}$ is also a consequence of a $\tau_{\infty}$-density argument on the $\mathrm{C}^{\infty}$ domain $\mathcal{D}_{\mathcal{S}}$, and establishes (3).

Proof of (4): To prove the isomorphism claim first note that, since $\overline{\mathcal{D}}_{\mathcal{S}}{ }^{\infty}$ is a $\mathrm{C}^{\infty}$ domain for $\overline{\mathcal{S}}$, compositions of operators in $\mathcal{L}_{\infty}$ are still operators in $\mathcal{L}_{\infty}$, so that it makes sense to consider a Lie algebra structure inside $\mathcal{L}_{\infty}$. To prove that the map $\left.B \longmapsto \bar{B}\right|_{\overline{\mathcal{D}}_{\mathcal{S}}}$ from $\mathcal{L}$ to $\mathcal{L}_{\infty}$ is a Lie algebra isomorphism it will first be proved that it is linear and that it preserves commutators. To these ends first note that, for every closable $B \in \mathcal{L}$, $\left.\bar{B}\right|_{\overline{\mathcal{D}}_{\mathcal{S}}} \subset \tilde{B}^{(1)}=\left.\bar{B}\right|_{\mathcal{D}_{1}}$ so, if $B=\sum_{k=1}^{d} b_{k} B_{k}$, then

$$
\left.\bar{B}\right|_{\overline{\mathcal{D}}_{\mathcal{S}}}{ }^{\infty}=\left.\sum_{k=1}^{d} b_{k} \overline{B_{k}}\right|_{\overline{\mathcal{D}}_{\mathcal{S}}}{ },
$$

by what was already noted at the very beginning of the proof. Therefore, if $C, D \in \mathcal{L}$ and $\mu \in \mathbb{C}$ with $C=\sum_{k=1}^{d} c_{k} B_{k}$ and $D=\sum_{k=1}^{d} d_{k} B_{k}$, then

$$
\left.\overline{C+\mu D}\right|_{\overline{\mathcal{D}}_{\mathcal{S}}}{ }^{d}=\left.\sum_{k=1}^{d}\left(c_{k}+\mu d_{k}\right) \overline{B_{k}}\right|_{\overline{\mathcal{D}}_{\mathcal{S}}}
$$

and, since $\left.\bar{C}\right|_{\overline{\mathcal{D}}_{\mathcal{S}}} ^{\infty}=\left.\sum_{k=1}^{d} c_{k} \overline{B_{k}}\right|_{\overline{\mathcal{D}}_{\mathcal{S}}} ^{\infty}$ and $\left.\bar{D}\right|_{\overline{\mathcal{D}}_{\mathcal{S}}} ^{\infty}=\left.\sum_{k=1}^{d} d_{k} \overline{B_{k}}\right|_{\overline{\mathcal{D}}_{\mathcal{S}}} ^{\infty}$, linearity follows. Preservation of commutators follows from a similar argument. That the map is a bijection is immediate, so $\mathcal{L}$ and $\mathcal{L}_{\infty}$ are indeed isomorphic. 


\section{Exponentiation Theorems}

\section{- The First Exponentiation Theorems}

Definition 2.6 (Exponentiable Lie Algebras of Linear Operators): Let $\mathcal{X}$ be a Hausdorff locally convex space, $\mathcal{D}$ a dense subspace of $\mathcal{X}$ and $\mathcal{L} \subseteq \operatorname{End}(\mathcal{D})$ a real finitedimensional Lie algebra of linear operators. Then, $\mathcal{L}$ is said to be exponentiable, or to exponentiate, if every operator in $\mathcal{L}$ is closable and if there exists a simply connected Lie group $G$ having a Lie algebra $\mathfrak{g}$ isomorphic to $\mathcal{L}$, via an isomorphism $\eta: \mathfrak{g} \ni X \longmapsto \eta(X) \in$ $\mathcal{L}$, and a strongly continuous locally equicontinuous representation $V: G \longrightarrow \mathcal{L}(\mathcal{X})$ such that $\mathcal{D} \subseteq C^{\infty}(V)$ and $d V(X)=\overline{\eta(X)}$, for all $X \in \mathfrak{g}$. If, moreover, every $d V(X)=\overline{\eta(X)}$, $X \in \mathfrak{g}$, is the generator of an equicontinuous one-parameter group (respectively, of a $\Gamma$ isometrically equicontinuous group, where $\Gamma$ is a fundamental system of seminorms for $\mathcal{X}$ ), it will be said that $\mathcal{L}$ exponentiates to a representation by equicontinuous one-parameter groups (respectively, exponentiates to a representation by $\Gamma$-isometrically equicontinuous one-parameter groups). If $\mathcal{X}$ is a Banach space, then an analogous terminology will be used: if $\mathcal{L}$ exponentiates and every $d V(X)=\overline{\eta(X)}, X \in \mathfrak{g}$, is the generator of a group of isometries, then it will be said that $\mathcal{L}$ exponentiates to a representation by isometries. Sometimes, $\mathfrak{g}$ and $\mathcal{L}$ will be identified, and the $\eta$ symbol will be omitted.

Below are two examples of exponentiable Lie algebras:

1. Consider the Schwartz functions space $\mathcal{S}\left(\mathbb{R}^{d}\right)$ as a dense subspace of $L^{2}\left(\mathbb{R}^{d}\right)$ and let

$$
\mathcal{L}:=\operatorname{span}_{\mathbb{R}}\left\{\left.\frac{\partial}{\partial x_{k}}\right|_{\mathcal{S}\left(\mathbb{R}^{d}\right)}: 1 \leq k \leq d\right\}
$$

be the finite-dimensional real Lie algebra which is generated (as a vector space) by the (weak) partial differentiation operators, with Dom $\frac{\partial}{\partial x_{k}}:=H^{1}\left(\mathbb{R}^{d}\right),{ }^{13}$ restricted to $\mathcal{S}\left(\mathbb{R}^{d}\right)$. The action $U$ of $\mathbb{R}^{d}$ on the space $L^{2}\left(\mathbb{R}^{d}\right)$ by translations defined by

$$
U(h): f \longmapsto f(\cdot+h), \quad h=\left(h_{k}\right)_{1 \leq k \leq d},
$$

is a strongly continuous representation of the commutative additive Lie group $\mathbb{R}^{d}$ by unitary operators on $L^{2}\left(\mathbb{R}^{d}\right)$. To prove $\mathcal{L}$ is exponentiable, it must first be noted that the Gårding domain of $U$ is contained in $\mathcal{S}\left(\mathbb{R}^{d}\right)$. In fact, if $\phi \in C_{c}^{\infty}\left(\mathbb{R}^{d}\right)$ and $f \in \mathcal{S}\left(\mathbb{R}^{d}\right)$, then

$$
f_{U}(\phi):=\int_{\mathbb{R}^{d}} \phi(h) U_{h}(f) d h
$$

\footnotetext{
${ }^{13} H^{1}\left(\mathbb{R}^{d}\right)$ denotes the Sobolev space of all functions in $L^{2}\left(\mathbb{R}^{d}\right)$ having weak first-order partial derivatives in $L^{2}\left(\mathbb{R}^{d}\right)$.
} 
belongs to $\mathcal{S}\left(\mathbb{R}^{d}\right),{ }^{14}$ so the Gårding domain

$$
D_{\mathbb{R}^{d}}(U):=\operatorname{span}_{\mathbb{C}}\left\{f_{U}(\phi):=\int_{\mathbb{R}^{d}} \phi(h) U_{h}(f) d h, \phi \in C_{c}^{\infty}\left(\mathbb{R}^{d}\right), f \in L^{2}\left(\mathbb{R}^{d}\right)\right\}
$$

is contained in $\mathcal{S}\left(\mathbb{R}^{d}\right)$. By [104, Theorem VIII.12, page 270], the closures of the operators

$$
\left.\frac{\partial}{\partial x_{k}}\right|_{D_{\mathbb{R}^{d}}(U)}, \quad 1 \leq k \leq d
$$

are the generators of the one-parameter groups $t \longmapsto U\left(t e_{k}\right)$, where $e_{k}$ is the $k$-th element of the canonical basis of $\mathbb{R}^{d}$. Therefore, since $D_{\mathbb{R}^{d}}(U) \subseteq \mathcal{S}\left(\mathbb{R}^{d}\right)$, it follows that

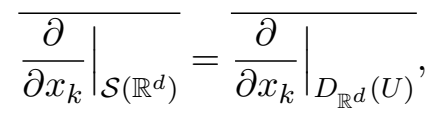

for all $1 \leq k \leq d$, since a self-adjoint operator does not have proper hermitian extensions. ${ }^{15}$ Also, because $C_{c}^{\infty}\left(\mathbb{R}^{d}\right)$ is dense in $H^{1}\left(\mathbb{R}^{d}\right)$ with respect to the usual Hilbert space topology of $H^{1}\left(\mathbb{R}^{d}\right)$ (see [83, Remark 10.31, page 291]), it follows that

$$
\overline{\frac{\partial}{\partial x_{k}}}=\overline{\left.\frac{\partial}{\partial x_{k}}\right|_{\mathcal{S}\left(\mathbb{R}^{d}\right)} .}
$$

Moreover, the Lie algebra of $\mathbb{R}^{d}$ is the real vector space generated by the set

$$
\left\{\left.\frac{\partial}{\partial x_{k}}\right|_{x=0}\right\}_{1 \leq k \leq d},
$$

which is sent onto $\mathcal{L}$ in an isomorphic way by the corresponding infinitesimal representation, introduced in Subsection 1.2.3 - note that the exponential map in question is defined by

$$
\left.\sum_{k=1}^{d} h_{k} \frac{\partial}{\partial x_{k}}\right|_{x=0} \longmapsto h=\left(h_{k}\right)_{1 \leq k \leq d}
$$

Also, $\mathcal{S}\left(\mathbb{R}^{d}\right)$ is contained in $C^{\infty}(U)$, by the maximality of $C^{\infty}(U)$ as a $\mathrm{C}^{\infty}$ domain, as it was seen in the constructive argument developed at the beginning of the chapter. Therefore, since $\mathbb{R}^{d}$ is simply connected (because it is a convex space, for example), $\mathcal{L}$ is exponentiable to a unitary representation of $\mathbb{R}^{d}$.

\footnotetext{
${ }^{14}$ This is because the convolution of two Schwartz functions is again a Schwartz function.

${ }^{15}$ To see this, suppose $A$ is self-adjoint and that $B$ is an hermitian extension of $A$ or, in other words, $B$ is a densely defined linear operator such that $B \subset B^{*}$. Then, $A \subset B \subset B^{*} \subset A^{*}$, so the fact that $A=A^{*}$ imposes $A=B$.
} 
2. Consider the $(2 n+1)$-dimensional real Lie algebra

$$
\mathcal{L}:=\operatorname{span}_{\mathbb{R}}\left\{\left.\frac{\partial}{\partial x_{k}}\right|_{\mathcal{S}\left(\mathbb{R}^{n}\right)},\left.i x_{k}\right|_{\mathcal{S}\left(\mathbb{R}^{n}\right)},\left.i\right|_{\mathcal{S}\left(\mathbb{R}^{n}\right)}: 1 \leq k \leq n\right\}
$$

generated (as a vector space) by the (weak) partial differentiation operators $\frac{\partial}{\partial x_{k}}$, the coordinate multiplication operators $x_{k}$ and the multiplication operator by the constant function $i$, all restricted to $\mathcal{S}\left(\mathbb{R}^{n}\right)$, where Dom $\frac{\partial}{\partial x_{k}}:=H^{1}\left(\mathbb{R}^{n}\right)$, Dom $x_{k}:=$ $\left\{f \in L^{2}\left(\mathbb{R}^{n}\right):\left(x \longmapsto x_{k} f(x)\right) \in L^{2}\left(\mathbb{R}^{n}\right)\right\}, 1 \leq k \leq n$, and Dom $i:=L^{2}\left(\mathbb{R}^{n}\right)$. Consider, also, the matrix group defined by

$$
H_{2 n+1}(\mathbb{R})=\left\{\left[\begin{array}{ccc}
1 & \mathbf{a} & c \\
0 & I_{n} & \mathbf{b} \\
0 & 0 & 1
\end{array}\right]: \mathbf{a} \in M_{1, n}(\mathbb{R}), \mathbf{b} \in M_{n, 1}(\mathbb{R}), c \in \mathbb{R}\right\}
$$

with the usual matrix multiplication, where $I_{n}$ is the identity matrix of $M_{n}(\mathbb{R})$. Then, it is possible to equip it with a smooth manifold structure, yielding a noncommutative $(2 n+1)$-dimensional Lie group known as the Heisenberg group of dimension $2 n+1$. Define the "partial group actions" on $L^{2}\left(\mathbb{R}^{n}\right)$ by

$$
\begin{aligned}
& {\left[\begin{array}{ccc}
1 & \mathbf{a} & 0 \\
0 & I_{n} & 0 \\
0 & 0 & 1
\end{array}\right] \longmapsto T_{\mathbf{a}}: f \longmapsto f(\cdot+\mathbf{a}),} \\
& {\left[\begin{array}{ccc}
1 & \mathbf{0} & 0 \\
0 & I_{n} & \mathbf{b} \\
0 & 0 & 1
\end{array}\right] \longmapsto M_{\mathbf{b}}: f \longmapsto e^{i\langle b, \cdot\rangle} f(\cdot)}
\end{aligned}
$$

and

$$
\left[\begin{array}{ccc}
1 & \mathbf{0} & c \\
0 & I_{n} & \mathbf{0} \\
0 & 0 & 1
\end{array}\right] \longmapsto \mathcal{M}_{c}: f \longmapsto e^{i c} f(\cdot) .
$$

These actions are strongly continuous unitary representations of their respective (commutative) Lie subgroups, and so is the representation $U$ of $H_{2 n+1}(\mathbb{R})$ defined by

$$
U_{\mathbf{a}, \mathbf{b}, c}:=U\left[\begin{array}{ccc}
1 & \mathbf{a} & c \\
0 & I_{n} & \mathbf{b} \\
0 & 0 & 1
\end{array}\right]: f \longmapsto\left(\mathcal{M}_{c} \circ M_{\mathbf{b}} \circ T_{\mathbf{a}}\right)(f),
$$

where $\left(\mathcal{M}_{c} \circ M_{\mathbf{b}} \circ T_{\mathbf{a}}\right)(f)(x)=e^{i c} e^{i\langle\mathbf{b}, x\rangle} f(x+\mathbf{a})$. The Lie algebra of $H_{2 n+1}(\mathbb{R})$ is (isomorphic to) the algebra of strictly upper triangular matrices

$$
\left\{\left[\begin{array}{ccc}
0 & \mathbf{a} & c \\
0 & 0_{n} & \mathbf{b} \\
0 & 0 & 0
\end{array}\right]: \mathbf{a} \in M_{1, n}(\mathbb{R}), \mathbf{b} \in M_{n, 1}(\mathbb{R}), c \in \mathbb{R}\right\}
$$


which is generated (as a vector space) by the set

$$
\left\{p_{j}:=\left[\begin{array}{ccc}
0 & e_{j}^{T} & 0 \\
0 & 0_{n} & \mathbf{0} \\
0 & 0 & 0
\end{array}\right], q_{k}:=\left[\begin{array}{ccc}
0 & \mathbf{0} & 0 \\
0 & 0_{n} & e_{k} \\
0 & 0 & 0
\end{array}\right], \mathbf{z}:=\left[\begin{array}{ccc}
0 & \mathbf{0} & 1 \\
0 & 0_{n} & \mathbf{0} \\
0 & 0 & 0
\end{array}\right], \quad 1 \leq j \leq n, 1 \leq k \leq n\right\} .
$$

These matrices are subject to the commutation relations $\left[p_{j}, q_{k}\right]=\delta_{j k} \mathbf{z},\left[p_{j}, z\right]=$ $\left[q_{k}, z\right]=0$. By the previous example, each $\left.\frac{\partial}{\partial x_{j}}\right|_{\mathcal{S}\left(\mathbb{R}^{n}\right)}$ is a pregenerator of $t \longmapsto$ $U\left(\exp t p_{j}\right)$. Also, the multiplication operators $x_{k}$ are all self-adjoint operators with their natural domains Dom $x_{k},{ }^{16}$ so $i x_{k}$ are the generators of the one-parameter unitary group representations $t \longmapsto U\left(\exp t q_{k}\right.$ ) (the same applies for the bounded multiplication operator by the constant function 1$)$. The exponential map is given by

$$
\left[\begin{array}{ccc}
0 & \mathbf{a} & c \\
0 & 0_{n} & \mathbf{b} \\
0 & 0 & 0
\end{array}\right] \longmapsto \exp \left[\begin{array}{ccc}
0 & \mathbf{a} & c \\
0 & 0_{n} & \mathbf{b} \\
0 & 0 & 0
\end{array}\right]=\sum_{m=0}^{+\infty} \frac{1}{m !}\left[\begin{array}{ccc}
0 & \mathbf{a} & c \\
0 & 0_{n} & \mathbf{b} \\
0 & 0 & 0
\end{array}\right]^{m}=\left[\begin{array}{ccc}
1 & \mathbf{a} & c+\frac{\langle\mathbf{a}, \mathbf{b}\rangle}{2} \\
0 & I_{n} & \mathbf{b} \\
0 & 0 & 1
\end{array}\right]
$$

so the infinitesimal representation sends each $p_{j}$ to $\left.\frac{\partial}{\partial x_{j}}\right|_{\mathcal{S}\left(\mathbb{R}^{n}\right)}, q_{k}$ to $\left.i x_{k}\right|_{\mathcal{S}\left(\mathbb{R}^{n}\right)}$ and $\mathbf{z}$ to $\left.i\right|_{\mathcal{S}\left(\mathbb{R}^{n}\right)}$ - note that $\mathcal{S}\left(\mathbb{R}^{n}\right) \subseteq C^{\infty}(U)$, by the same reason of the previous example. Furthermore, $\mathcal{S}\left(\mathbb{R}^{n}\right)$ is a core for all of the generators: it is a core for the closure of the (weak) partial differentiation operators, by the previous example, and a core for the multiplication operators $x_{k}$, because $\mathcal{S}\left(\mathbb{R}^{n}\right) \subseteq$ Dom $x_{k}$ is dense in $L^{2}\left(\mathbb{R}^{n}\right)$ and is left invariant by $U$ (see [30, Corollary 3.1.7, page 167] or the more general Lemma 1.2.4.3, of Chapter 1$)$. Because $H_{2 n+1}(\mathbb{R})$ is homeomorphic to $\mathbb{R}^{2 n+1}$ and simple connectedness is a topological property (in other words, it is preserved by homeomorphisms), this shows $\mathcal{L}$ exponentiates to a unitary representation of the simply connected Lie group $H_{2 n+1}(\mathbb{R})$. It should also be noted that the commutation relations

$$
\left[\left.\frac{\partial}{\partial x_{j}}\right|_{\mathcal{S}\left(\mathbb{R}^{n}\right)},\left.i x_{k}\right|_{\mathcal{S}\left(\mathbb{R}^{n}\right)}\right]=\left.\delta_{j k} i\right|_{\mathcal{S}\left(\mathbb{R}^{n}\right)},\left[\left.\frac{\partial}{\partial x_{k}}\right|_{\mathcal{S}\left(\mathbb{R}^{n}\right)},\left.i\right|_{\mathcal{S}\left(\mathbb{R}^{n}\right)}\right]=\left[\left.i x_{k}\right|_{\mathcal{S}\left(\mathbb{R}^{n}\right)},\left.i\right|_{\mathcal{S}\left(\mathbb{R}^{n}\right)}\right]=0
$$

hold, so this Lie algebra representation is a realization of the Canonical Commutation Relations (CCR) by unbounded operators on $L^{2}\left(\mathbb{R}^{n}\right)$. See also [118, Example 10.2.14, page 271], [73, pages 31 and 32] and [42, page 248].

A few words about Definition 2.6 need to be said. In finite-dimensional representation theory - meaning $\mathcal{X}$ is finite-dimensional - every real finite-dimensional Lie algebra of linear operators is exponentiable. One way to arrive at this conclusion is to first consider a simply

\footnotetext{
${ }^{16}$ See [32, XIII) 2., page 19].
} 
connected Lie group $G$ having a Lie algebra isomorphic to $\mathcal{L}$ ( $G$ is unique, up to isomorphism), which always exists, by a theorem - this follows, for example, from Lie's Third Theorem - see [67, Theorem 9.4.11, page 334] or [67, Remark 9.5.12, page 341] - combined with [67, Proposition 9.5.1, page 335]. Then, using the Baker-Campbell-Hausdorff Formula, one manages to construct a local homomorphism ${ }^{17}$ from $G$ to $\mathcal{L}(\mathcal{X})$ which, by simple connectedness of $G$, is always extendable to a global homomorphism $\tilde{\phi}: G \longrightarrow \mathcal{L}(\mathcal{X})$, by the so-called Monodromy Principle (see [67, Proposition 9.5.8, page 339] and [1, Theorem 1.4.5, page 50]). Proceeding in this way, a group homomorphism $\tilde{\phi}: G \longrightarrow \mathcal{L}(\mathcal{X})$ with the desired properties may be finally obtained [67, Theorem 9.5.9, page 340]. For a brief discussion of exponentiability on finite-dimensional spaces, see also [73, page 271].

Real finite-dimensional Lie algebras can always be represented faithfully as Lie subalgebras of $\mathfrak{g l}_{n}(\mathbb{R})$, for some $n \in \mathbb{N}, n \geq 1\left(\mathfrak{g l}_{n}(\mathbb{R})\right.$ denotes the space of all linear transformations on the $n$-dimensional real vector space $V$ ) - this result is known as Ado's Theorem [67, Theorem 7.4.1, page 189]. The Lie group counterpart is much more subtle: one noteworthy fact is that not every Lie group admits a non-trivial unitary strongly continuous finite-dimensional representation. To illustrate this fact, the following theorem will be demonstrated: ${ }^{18}$

Theorem 2.6.1: If $n \in \mathbb{N}, n \geq 1, G$ is a non-compact connected Lie group which is simple as an abstract group (in other words, it does not have any proper normal subgroups) and $\pi: G \longrightarrow G L_{n}(V, \mathbb{C})$ is a strongly continuous (and, therefore, it is a smooth map) finite-dimensional unitary representation over the $n$-dimensional complex vector space $V$, then $\pi$ is trivial - in other words, $\pi(g)$ is the identity operator on $V$, for every $g \in G .^{19}$

Proof of Theorem 2.6.1: Non-triviality of Ker $\pi$ implies that it must be all of $G$, by the hypothesis, so suppose $\pi$ is faithful. By [67, Corollary 14.5.7, page 556$],{ }^{20} \pi[G]$ is a closed subgroup of $G L_{n}(V, \mathbb{C})$, so it must be a Lie group with the induced topology of $G L_{n}(V, \mathbb{C})$, by [67, Corollary 9.2.17, page 306] and [67, Proposition 9.3.9, page 322]. Since every smooth group homomorphism between Lie groups has constant rank, ${ }^{21}$ it must be a

\footnotetext{
${ }^{17} \phi$ is said to be a local homomorphism from $G$ to $\mathcal{L}(\mathcal{X})$ if there exists an open symmetric connected neighborhood of the identity $\Omega$ such that $\phi: \Omega \longrightarrow \mathcal{L}(\mathcal{X})$ is a map satisfying $\phi(g h)=\phi(g) \phi(h)$, for all $g, h$ in $\Omega$ for which $g h$ also belongs to $\Omega$.

${ }^{18}$ This argument was developed based on the article "Representation theory of the Lorentz Group" of Wikipedia - more precisely, the section "Non-unitarity" - and on the discussion thread "Unitary representations of non-compact Lie groups", at "math.stackexchange.com" - see the answers given by Berni Waterman; see also Valter Moretti's answers in the thread "On finite-dimensional unitary representations of non-compact Lie groups", of "physics.stackexchange.com".

${ }^{19}$ A non-abelian abstract group $G$ is said to be simple if it is has no normal subgroups other than $\{e\}$ and $G$, itself, where $e$ is the identity of $G$. Here, $G L_{n}(V, \mathbb{C})$ denotes the group of all linear automorphisms of $V$.

${ }^{20}\left[67\right.$, Corollary 14.5.7, page 556]: If $G$ is a semisimple connected Lie group and $\pi: G \longrightarrow G L_{n}(V, \mathbb{C})$ is a strongly continuous finite-dimensional representation, then $\pi[G]$ is closed in $G L_{n}(V, \mathbb{C})$.

${ }^{21}$ For a proof of this fact, see Theorem 18 in the notes of professor Gye-Seon Lee [82].
} 
diffeomorphism, by the Global Rank Theorem for smooth manifolds. Now, since $\phi[G]$ is contained in the compact group of unitary matrices $U(n)$ (compactness of such Lie group follows from Heine-Borel Theorem, since $U(n)$ is a closed and bounded subset of $M_{n}(\mathbb{C})$ ), it must also be compact, so continuity of $\phi^{-1}$ implies $\phi^{-1}[\phi[G]]=G$ is also compact, which contradicts the non-compactness hypothesis on $G$. Hence, $\phi$ must the trivial homomorphism.

With a small adaptation of the proof above, the following corollary is obtained:

Corollary 2.6.2: If $G$ is a semisimple non-compact connected Lie group then it does not possess any faithful finite-dimensional strongly continuous unitary representations into $G L_{n}(V, \mathbb{C})$, for any $n \in \mathbb{N}, n \geq 1 .^{22}$

As an application of the above corollary, consider the matrix Lie group $S L_{n}(\mathbb{R})$ of all matrices in $\mathfrak{g l}_{n}(\mathbb{R})$ having determinant equal to 1 , where $n \geq 2$. By [67, Proposition 17.2.3, page 611$], S L_{n}(\mathbb{R})$ is connected and semisimple. To see it is not compact, it is sufficient to see that it contains the set $\left\{\left(a_{i j}^{(m)}\right)_{1 \leq i, j \leq n}\right\}_{m \in \mathbb{N} \backslash\{0\}}$ of matrices defined for each $m \in \mathbb{N}$ by $a_{11}^{(m)}:=m, a_{22}:=1 / m, a_{k k}^{(m)}:=1$, for $n \geq k>2$, and $a_{i j}^{(m)}:=0$, otherwise. Therefore, $S L_{n}(\mathbb{R})$ is not bounded, so it cannot be compact. Hence, by Corollary 2.6.2, $S L_{n}(\mathbb{R})$ does not have any faithful finite-dimensional strongly continuous unitary representations.

The restricted Lorentz group, also referred to as the group of proper orthochronous Lorentz transformations, $S O(1,3)$, or $S O(3,1)$ (for the precise definition of this group, which has small variations in the literature, see [67, page 29], or [52, Chapter 7: Special Relativity]), is a Lie group which is isomorphic to the (real) Lie group

$$
P S L_{2}(\mathbb{C}):=S L_{2}(\mathbb{C}) /\{-1,1\}
$$

where $S L_{2}(\mathbb{C})$ denotes the group of $2 \times 2$ complex matrices of determinant $1 .{ }^{23}$ Now, $S L_{2}(\mathbb{C})$ is connected (in fact, $S L_{2}(\mathbb{C})$ is simply connected, by [67, Proposition 17.2.1(2), page 610], so $P S L_{2}(\mathbb{C})$ is the image of a continuous map defined on a connected topological space), simple ${ }^{24}$ and non-compact Lie group, since $S L_{2}(\mathbb{C})$ is not compact (see the argument of non-compactness given above, for $S L_{2}(\mathbb{R})$ ). Therefore, the only finite-dimensional unitary representation of the restricted Lorentz group is the trivial one, by Theorem 2.6.1.

Surprisingly, on the other hand, if $G$ is a compact Lie group, there always exists a faithful finite-dimensional strongly continuous representation $\pi: G \longrightarrow G L_{n}(V, \mathbb{C})$, for

\footnotetext{
${ }^{22}$ A connected Lie group $G$ is said to be semisimple if its Lie algebra is semisimple; a Lie algebra $\mathfrak{g}$ is said to be semisimple if it is a direct sum of simple ideals.

${ }^{23}$ See [56] (the restricted Lorentz group is sometimes denoted by $S O_{0}(1,3)$ and, sometimes, by $S O_{0}(3,1)$, in this reference) and [37, Theorem 3.4, page 24].

${ }^{24} \mathrm{~A}$ proof of this fact, by Keith Conrad, may be found in [40].
} 
some $n \geq 1$, as a consequence of Peter-Weyl Theorem (see [77, Corollary 4.22, page 248]). ${ }^{25}$

Hopefully, the discussion above regarding Lie group representations motivated a little bit more the efforts that will be employed in this section.

To obtain the first exponentiation theorem of this section (Theorem 2.7), which is an "equicontinuous locally convex version" of [74, Theorem 9.2, page 197], another theorem of [74] is going to be needed:

[74, Theorem 9.1, page 196]: Let $\mathcal{X}$ be a Hausdorff locally convex space, ${ }^{26} \mathcal{D}$ a dense subspace of $\mathcal{X}$ and $\mathcal{L} \subseteq \operatorname{End}(\mathcal{D})$ a finite dimensional real Lie algebra of linear operators. Suppose $\mathcal{L}$ is generated, as a Lie algebra, by a finite set $\mathcal{S}$ of infinitesimal pregenerators of strongly continuous locally equicontinuous groups (those are abbreviated as cle groups, in [74] - see page 62; see also page 178 of this same reference), where $t \longmapsto V(t, \bar{A})$ denotes the group generated by $\bar{A}$, for all $A \in \mathcal{S}$. If the following two conditions are satisfied then, $\mathcal{L}$ exponentiates to a strongly continuous representation of a Lie group:

1. the domain $\mathcal{D}$ is left invariant by the operators $\{V(t, \bar{A}): A \in \mathcal{S}, t \in \mathbb{R}\}$;

2. for each $x \in \mathcal{D}$ and each pair of elements $A, B \in \mathcal{S}$ there is an open interval (which may depend on $x, A$ and $B$ ) such that the function $t \longmapsto B V(t, \bar{A})$ is bounded in $I$.

Theorem 2.7: Let $(\mathcal{X}, \tau)$ be a complete Hausdorff locally convex space, $\mathcal{D}$ a dense subspace of $\mathcal{X}$ and $\mathcal{L} \subseteq \operatorname{End}(\mathcal{D})$ a finite dimensional real Lie algebra of linear operators. Assume the same hypotheses of Theorem 2.5. Then, $\mathcal{L}$ exponentiates.

Proof of Theorem 2.7: Using the notations and the results of the previous section, consider the complete Hausdorff locally convex space $\overline{\mathcal{D}}_{\mathcal{S}}{ }^{\infty}$, constructed in Theorem 2.5, which is a $\mathrm{C}^{\infty}$ domain for $\overline{\mathcal{S}}$ that contains $\mathcal{D}$ and is left invariant by the operators $\{V(t, \bar{A}): A \in \mathcal{S}, t \in \mathbb{R}\}$. The estimates

$$
p(B V(t, \bar{A}) x) \leq|B|_{1} \rho_{p, 1}(V(t, \bar{A}) x)
$$

for all $p \in \Gamma_{A}, x \in \mathcal{D}$ and $A, B \in \mathcal{S}$, follow from the usual arguments and extend by $\tau_{1}$-density to

$$
p\left(\left.\bar{B}\right|_{\overline{\mathcal{D}}_{\mathcal{S}}} V(t, \bar{A}) x\right) \leq|B|_{1} \rho_{p, 1}(V(t, \bar{A}) x)
$$

\footnotetext{
${ }^{25}$ Peter-Weyl Theorem is valid for compact topological groups - they do not need to have a Lie group structure. See [77, Theorem 4.20, page 245] and [113, Theorem 7.5.14, page 470].

${ }^{26}$ Locally convex spaces are automatically assumed to be Hausdorff in [74] (see the second paragraph of Appendix A, of the reference).
} 
for all $x \in{\overline{\mathcal{D}_{\mathcal{S}}}}^{\infty}$, by $\tau_{1}$-continuity of $\left.V(t, \bar{A})\right|_{\mathcal{D}_{1}},\left(\tau_{1} \times \tau_{1}\right)$-closedness of $\bar{B}^{\tau_{1}}$ and $\left.\bar{B}\right|_{\overline{\mathcal{D}}_{\mathcal{S}}} \subset$ $\bar{B}^{\tau_{1}}=\left.\bar{B}\right|_{\mathcal{D}_{1}}$. This proves the existence of a non-empty open interval on which the function

$$
\left.I \ni t \longmapsto \bar{B}\right|_{\overline{\mathcal{D}}_{\mathcal{S}}} V(t, \bar{A}) x
$$

is bounded. Also,

$$
\bar{A}=\overline{\left.\bar{A}\right|_{\overline{\mathcal{D}}_{\mathcal{S}}}}, \quad A \in \mathcal{S},
$$

so each $\left.\bar{A}\right|_{\overline{\mathcal{D}}_{\mathcal{S}}}$ is a pregenerator of an equicontinuous group on $\mathcal{X}$. Applying [74, Theorem 9.1, page 196], above, with $\mathcal{D}$ replaced by $\overline{\mathcal{D}}_{\mathcal{S}}{ }^{\infty}$, yields that $\mathcal{L}_{\infty}$ is exponentiable. Since item 4 of Theorem 2.5 established that $\mathcal{L}_{\infty}$ and $\mathcal{L}$ are isomorphic, the result follows.

Since every basis of a finite-dimensional Lie algebra $\mathcal{L}$ is a set of generators for $\mathcal{L}$, the following corollary is also true:

Corollary 2.8: Assume the same hypotheses of Theorem 2.7 are valid, with $\mathcal{S}$ replaced by $\left(B_{k}\right)_{1 \leq k \leq d}$. Then, $\mathcal{L}$ exponentiates. If the stronger hypothesis that there exists a fundamental system of seminorms $\Gamma$ for $\mathcal{X}$ such that the operators $B_{k}$ are all $\Gamma$-conservative (that is, they are conservative with respect to the same $\Gamma$ ), then $\mathcal{L}$ exponentiates to a representation by $\Gamma$-isometrically equicontinuous one-parameter groups.

Proof of Corollary 2.8: Let $G \ni g \longmapsto V(g) \in \mathcal{L}(\mathcal{X})$ be the underlying Lie group representation, $\mathfrak{g}$ the Lie algebra of $G$ and $\eta: \mathfrak{g} \longrightarrow \mathcal{L} \subseteq \operatorname{End}(\mathcal{D})$ be the Lie algebra representation as in Definition 2.6. Suppose that the stronger hypotheses of Corollary 2.8 are valid. Then, formula (7) at [126, page 248] says that

$$
V\left(\exp t \eta^{-1}\left(B_{k}\right)\right) x=\lim _{n \rightarrow+\infty} \exp \left(t \overline{B_{k}}\left(I-\frac{1}{n} \overline{B_{k}}\right)^{-1}\right) x, \quad t \in[0,+\infty), x \in \mathcal{X} .
$$

Then, a repetition of the argument in the last paragraph of the proof of Theorem 1.4.3 shows that each $t \longmapsto V\left(\exp t \eta^{-1}\left(B_{k}\right)\right)$ is a $\Gamma$-isometrically equicontinuous group. Now, there exist $d$ real-valued analytic functions $\left\{t_{k}\right\}_{1 \leq k \leq d}$ defined on a neighborhood $\Omega$ of the identity of $G$ such that $g \longmapsto\left(t_{k}(g)\right)_{1 \leq k \leq d}$ maps $\Omega$ diffeomorphically onto a neighborhood of the origin of $\mathbb{R}^{d}$, with

$$
g=\exp \left(t_{1}(g) \eta^{-1}\left(B_{1}\right)\right) \ldots \exp \left(t_{k}(g) \eta^{-1}\left(B_{k}\right)\right) \ldots \exp \left(t_{d}(g) \eta^{-1}\left(B_{d}\right)\right), \quad g \in \Omega .
$$

Therefore, connectedness of $G$ guarantees that the group generated by $\Omega$ is all of $G$, so every $g \in G$ can be written as $g=\prod_{j=1}^{n(g)} \exp s_{j} \eta^{-1}\left(B_{k_{j}}\right)$, where $s_{j} \in \mathbb{R}$ and $n(g) \in \mathbb{N}$. Therefore, the group property of $V$ gives $p(V(g) x)=p(x)$, for all $p \in \Gamma, g \in G$ and $x \in \mathcal{X}$ so, in particular, $t \longmapsto V(\exp t X)$ is a $\Gamma$-isometrically equicontinuous group, for all $X \in \mathfrak{g}$. 
This shows that every $d V(X)=\overline{\eta(X)}, X \in \mathfrak{g}$, is the generator of a $\Gamma$-isometrically equicontinuous group, so $\mathcal{L}$ exponentiates to a representation by $\Gamma$-isometrically equicontinuous one-parameter groups.

The next theorem is a version of [59, Theorem 3.1] for pregenerators of equicontinuous groups on complete Hausdorff locally convex spaces. It is a $d$-dimensional noncommutative version of Lemma 1.4.3, in the sense that it guarantees exponentiability of a $d$-dimensional and (possibly) noncommutative Lie algebra of linear operators, instead of a 1-dimensional one:

Theorem 2.9: Let $(\mathcal{X}, \tau)$ be a complete Hausdorff locally convex space, $\mathcal{D}$ a dense subspace of $\mathcal{X}$ and $\mathcal{L} \subseteq \operatorname{End}(\mathcal{D})$ a finite dimensional real Lie algebra of linear operators defined on $\mathcal{D}$. Assume that the following hypotheses are valid:

1. $\mathcal{L}$ is generated, as a Lie algebra, by a finite set $\mathcal{S}$ of infinitesimal pregenerators of equicontinuous groups. For each $A \in \mathcal{S}$, let $\Gamma_{A}$ be the fundamental system of seminorms for $\mathcal{X}$ constructed right after Lemma 1.3.1, in Section 1.3, with respect to which the operator $\bar{A}$ has the $(K I P)$, is $\Gamma_{A}$-conservative and $V(\cdot, \bar{A})$ is $\Gamma_{A}$-isometrically equicontinuous;

2. $\mathcal{L}$ possesses a basis $\left(B_{k}\right)_{1 \leq k \leq d}$ formed by closable elements having the (KIP) with respect to

$$
\Gamma_{\mathcal{S}}:=\bigcup_{A \in \mathcal{S}} \Gamma_{A}
$$

3. for each fixed $A \in \mathcal{S}$, every element of $\mathcal{D}$ is a $\tau$-projective analytic vector for $A$.

Then, $\mathcal{L}$ exponentiates.

Proof of Theorem 2.9: Fix $A \in \mathcal{S}$. The proof will be divided in steps:

1 Each $x \in \mathcal{D}$ is a $\tau_{1}$-projective analytic vector for $\boldsymbol{A}$ : fix $p \in \Gamma_{A}$ and $x \in \mathcal{D}$. Since $B_{k}(x)$ is a $\tau$-projective analytic vector for $A$, for all $1 \leq k \leq d$ (remember $\left.B_{0}:=I\right)$, there exist $C_{x, p}, s_{x, p}>0$ such that

$$
p\left(A^{n} B_{k}(x)\right) \leq C_{x, p} s_{x, p}^{n} n !, \quad n \in \mathbb{N}, 1 \leq k \leq d .
$$

In fact, if for each fixed $0 \leq k \leq d$, a certain $r_{x, p}(k)>0$ satisfying

$$
\sum_{j=0}^{+\infty} \frac{p\left(A^{j} B_{k}(x)\right)}{j !} r_{x, p}(k)^{j}<\infty
$$


is chosen, it suffices to make

$$
C_{x, p}:=\max \left\{\sum_{j=0}^{+\infty} \frac{p\left(A^{j} B_{k}(x)\right)}{j !} r_{x, p}(k)^{j}, 0 \leq k \leq d\right\}
$$

and

$$
s_{x, p}:=\max \left\{\frac{1}{r_{x, p}(k)}: 0 \leq k \leq d\right\} .
$$

Hence, if $C=\sum_{j=1}^{d} c_{j} B_{j}$, then

$$
p\left(A^{n}(C(x))\right) \leq \sum_{k=1}^{d}\left|c_{k}\right| p\left(A^{n} B_{k}(x)\right) \leq|C|_{1} C_{x, p} s_{x, p}^{n} n !,
$$

for all $n \in \mathbb{N}$. This implies

$$
p\left(A^{n}(\operatorname{ad} A)^{m}(B)(x)\right) \leq\left|(\operatorname{ad} A)^{m}(B)\right|{ }_{1} C_{x, p} s_{x, p}^{n} n ! \leq\|\operatorname{ad} A\|^{m}|B|{ }_{1} C_{x, p} s_{x, p}^{n} n !,
$$

for all $m, n \in \mathbb{N}$ and $B \in \mathcal{L}$. One can prove by induction that

$$
B A^{n}(x)=\sum_{j=0}^{n}\left(\begin{array}{l}
n \\
j
\end{array}\right)(-1)^{n-j} A^{j}(\operatorname{ad} A)^{n-j}(B)(x), \quad n \in \mathbb{N}, B \in \mathcal{L},
$$

so that

$$
\begin{gathered}
p\left(B A^{n}(x)\right) \leq \sum_{j=0}^{n}\left(\begin{array}{l}
n \\
j
\end{array}\right) p\left(A^{j}(\operatorname{ad} A)^{n-j}(B)(x)\right) \\
\leq \sum_{j=0}^{n}\left(\begin{array}{c}
n \\
j
\end{array}\right)\|\operatorname{ad} A\|^{n-j}|B|_{1} C_{x, p} s_{x, p}^{j} j !, \quad p \in \Gamma_{A}, x \in \mathcal{D} .
\end{gathered}
$$

Applying the inequality just obtained, it follows that for all $z \in \mathbb{C}$,

$$
\begin{gathered}
\sum_{n=0}^{+\infty} \frac{p\left(B A^{n}(x)\right)}{n !}|z|^{n} \leq|B|{ }_{1} C_{x, p} \sum_{n=0}^{+\infty}|z|^{n} \sum_{k=0}^{n}\left(\begin{array}{l}
n \\
k
\end{array}\right)\|\operatorname{ad} A\|^{n-k} s_{x, p}^{k} \frac{k !}{n !} \\
\leq|B|{ }_{1} C_{x, p} \sum_{n=0}^{+\infty}|z|^{n} \sum_{k=0}^{n}\left(\begin{array}{l}
n \\
k
\end{array}\right)\|\operatorname{ad} A\|^{n-k} s_{x, p}^{k}=|B|{ }_{1} C_{x, p} \sum_{n=0}^{+\infty}\left(\|\operatorname{ad} A\|+s_{x, p}\right)^{n}|z|^{n},
\end{gathered}
$$

and the latter series converges for all $z \in \mathbb{C}$ satisfying $\left(\|\operatorname{ad} A\|+s_{x, p}\right)|z|<1$. In particular, substituting $B$ by $B_{k}$ and taking the maximum over $0 \leq k \leq d$ proves the desired analyticity result. 


\section{For every $p \in \Gamma_{A}$ and every $\lambda \in \mathrm{C}$ satisfying $|\operatorname{Re} \lambda|>\|\tilde{\operatorname{ad}} \boldsymbol{A}\|$,

$$
\rho_{p, 1}((\lambda I-A)(x)) \geq(|\operatorname{Re} \lambda|-\|\tilde{\operatorname{ad}} A\|) \rho_{p, 1}(x), \quad x \in \mathcal{D}:
$$

By the choice of $\Gamma_{A}$, one sees that, for all $\lambda \in \mathbb{C} \backslash i \mathbb{R}$, the resolvent operator $\mathrm{R}(\lambda, \bar{A})$ satisfies the bounds

$$
p(\mathrm{R}(\lambda, \bar{A})(x)) \leq \frac{1}{|\operatorname{Re} \lambda|} p(x), \quad p \in \Gamma_{A}, x \in \mathcal{X} .
$$

Then, a repetition of the argument at the beginning of the proof of Theorem 2.4 gives the desired estimates.

Now, since the basis elements possess the $(\mathrm{KIP})$ with respect to $\Gamma_{\mathcal{S}}$, the operator

$$
A: \mathcal{D} \subseteq \mathcal{D}_{1} \longrightarrow \mathcal{D}_{1}
$$

is a $\tau_{1}$-densely defined $\left(\tau_{1} \times \tau_{1}\right)$-closable linear operator on $\mathcal{D}_{1}$ which possesses the $(\mathrm{KIP})$ with respect to $\Gamma_{A, 1} \subseteq \Gamma_{\mathcal{S}}$, by the same argument explained at the beginning of Theorem 2.4. Therefore, the induced linear operator

$$
A_{\rho_{p, 1}}: \pi_{\rho_{p, 1}}[\mathcal{D}] \longrightarrow\left(\mathcal{D}_{1}\right)_{\rho_{p, 1}}, \quad A_{\rho_{p, 1}}\left([x]_{\rho_{p, 1}}\right)=\left[\bar{A}^{\tau_{1}}(x)\right]_{\rho_{p, 1}},
$$

is well-defined for all $\rho_{p, 1} \in \Gamma_{A, 1}$, and $\pi_{\rho_{p, 1}}[\mathcal{D}]$ is a $\|\cdot\|_{\rho_{p, 1}}$-dense set of analytic vectors for $A_{\rho_{p, 1}}$, by item 1 , above. Furthermore, the inequality proved in item 2 implies

$$
\rho_{p, 1}\left((\lambda I-A)^{n}(x)\right) \geq(|\operatorname{Re} \lambda|-\|\tilde{a d} A\|)^{n} \rho_{p, 1}(x), \quad|\operatorname{Re} \lambda|>\|\tilde{a d} A\|,
$$

for all $\rho_{p, 1} \in \Gamma_{A, 1}, n \in \mathbb{N}, x \in \mathcal{D}$. Therefore, [111, Theorem 1] becomes applicable, and the operators $A_{\rho_{p, 1}}$ are infinitesimal pregenerators of strongly continuous groups $t \longmapsto$ $V\left(t, \overline{A_{\rho_{p, 1}}}\right)$ on $\left(\mathcal{D}_{1}\right)_{\rho_{p, 1}}$ which satisfy

$$
\left\|V\left(t, \overline{A_{\rho_{p, 1}}}\right) x_{\rho_{p, 1}}\right\|_{\rho_{p, 1}} \leq \exp (\|\tilde{\operatorname{ad}} A\||t|)\left\|x_{\rho_{p, 1}}\right\|_{\rho_{p, 1}}, \quad x_{\rho_{p, 1}} \in\left(\mathcal{D}_{1}\right)_{\rho_{p, 1}} .
$$

Hence, by a classical generation-type theorem for groups (see the Generation Theorem for Groups in [50, page 79], mentioned in Section 1.2) it follows that, for each $p \in \Gamma$ and every $\lambda \in \mathbb{C}$ satisfying $|\operatorname{Re} \lambda|>\|\tilde{a d} A\|$, the resolvent operators $\mathrm{R}\left(\lambda, \overline{A_{\rho_{p, 1}}}\right)$ are well-defined (in other words, $\lambda \in \rho\left(\overline{A_{\rho_{p, 1}}}\right)$, for all $\rho_{p, 1} \in \Gamma_{A, 1}$ and all $\lambda \in \mathbb{C}$ satisfying $\left.|\operatorname{Re} \lambda|>\|\tilde{a d} A\|\right)$ and the ranges

$$
\left(\lambda I-A_{\rho_{p, 1}}\right)\left[\pi_{\rho_{p, 1}}[\mathcal{D}]\right]
$$

are dense in their respective spaces $\left(\mathcal{D}_{1}\right)_{\rho_{p, 1}}$. In particular, they are dense in $\pi_{\rho_{p, 1}}[\mathcal{D}]$, for each $\rho_{p, 1} \in \Gamma_{A, 1} \cdot{ }^{27}$ Just as it was argued at the beginning of the proof of Lemma 1.4.3 (remember $\Gamma_{A, 1}$ is a saturated family of seminorms), one concludes $\operatorname{Ran}(\lambda I-A)$ is $\tau_{1}$-dense

\footnotetext{
${ }^{27}$ This follows from the fact that $\lambda I-\bar{A}_{\rho_{p, 1}}$ is surjective and from $\operatorname{Ran}\left(\lambda I-\overline{A_{\rho_{p, 1}}}\right)=\overline{\operatorname{Ran}\left(\lambda I-A_{\rho_{p, 1}}\right)}$.
} 
in $\mathcal{D}$ if $|\operatorname{Re} \lambda|>\|\tilde{a d} A\|$, for all $A \in \mathcal{S}$. Therefore, since $A \in \mathcal{S}$ is arbitrary, Theorem 2.7 guarantees $\mathcal{L}$ is exponentiable.

Just as in the case of Corollary 2.8, the following is also true:

Corollary 2.10: Assume the same hypotheses of Theorem 2.9 are valid, with $\mathcal{S}$ replaced by $\left(B_{k}\right)_{1 \leq k \leq d}$. Then, $\mathcal{L}$ exponentiates. If the stronger hypothesis that there exists a fundamental system of seminorms $\Gamma$ for $\mathcal{X}$ such that the operators $B_{k}$ are all $\Gamma$-conservative (that is, they are conservative with respect to the same $\Gamma$ ), then $\mathcal{L}$ exponentiates to a representation by $\Gamma$-isometrically equicontinuous one-parameter groups.

\section{- Strongly Elliptic Operators - Sufficient Conditions for Exponentiation}

An important concept which will permeate the next theorems is that of a strongly elliptic operator.

Definition (Strongly Elliptic Operators): Let $\mathcal{X}$ be a complete Hausdorff locally convex space, $G$ a Lie group with Lie algebra $\mathfrak{g}$, with $\left(X_{k}\right)_{1 \leq k \leq d}$ being an ordered basis for $\mathfrak{g}$, and $V: G \longrightarrow \mathcal{L}(\mathcal{X})$ a strongly continuous locally equicontinuous representation, where

$$
\partial V: \mathfrak{g} \ni X \longmapsto \partial V(X) \in \operatorname{End}\left(C^{\infty}(V)\right)
$$

is the induced Lie algebra representation. Assume, without loss of generality, that $\partial V$ is faithful (in other words, that it is an injective map). It is said that an operator

$$
H_{m}:=\sum_{|\alpha| \leq m} c_{\alpha} \partial V\left(X^{\alpha}\right)
$$

of order ${ }^{28} m$ belonging to the complexified enveloping algebra of $\mathfrak{g},(\mathfrak{U}[\partial V[\mathfrak{g}]])_{\mathbb{C}}:=\mathfrak{U}[\partial V[\mathfrak{g}]]+$ $i \mathfrak{U}[\partial V[\mathfrak{g}]]$, is elliptic if the polynomial function

$$
P_{m}(\xi):=\sum_{|\alpha|=m} c_{\alpha} \xi^{\alpha}
$$

on $\mathbb{R}^{d}$, called the principal part of $H_{m}$, satisfies $P_{m}(\xi) \neq 0$, if $\xi \neq 0$. This definition is basis independent - see [109, page 21]. Finally, an elliptic operator is said to be strongly elliptic if

$$
R e(-1)^{m / 2} \sum_{|\alpha|=m} c_{\alpha} \xi^{\alpha}>0
$$

\footnotetext{
${ }^{28}$ Remember the notation introduced in Subsection 1.2.4.
} 
for all $\xi \in \mathbb{R}^{d} \backslash\{0\}$ - see [109, page 28]. The prototypical example of a strongly elliptic operator is the "minus Laplacian" operator

$$
-\sum_{k=1}^{d}\left[\partial V\left(X_{k}\right)\right]^{2}
$$

For a matter of terminological convenience, in the future (Theorem 2.14), the same definition which was just made for elliptic and strongly elliptic operators will be extended to (complexifications of) universal enveloping algebras of operators which are not necessarily associated with a strongly continuous Lie group representation.

To illustrate the usefulness of the hypothesis of strong ellipticity, two examples coming from the realm of PDEs will be given: ${ }^{29}$

1. Example taken from [96]: Let $U \subseteq \mathbb{R}^{n}$ be a bounded open set with boundary of class $\mathrm{C}^{1}$ and define, for each $j \in \mathbb{N}$, the spaces (i) $H^{j, 2}(U)$ of functions in $L^{2}(U)$ having weak derivatives up to order $j$ in $L^{2}(U)$ and (ii) $H_{0}^{j, 2}(U)$, defined as the completion of the space $C_{c}^{\infty}(U)$ relatively to the norm $\|\cdot\|_{j}$ induced by the inner product

$$
\langle f, g\rangle_{j}:=\sum_{0 \leq|\alpha| \leq j} \int_{U} D^{\alpha} f(x) \overline{D^{\alpha} g(x)} d \mu(x),
$$

where $D^{\alpha}$ and $D^{\beta}$ denote (weak) partial differentiation operators. Note that $\langle\cdot, \cdot\rangle:=$ $\langle\cdot, \cdot\rangle_{0}$ and $\|\cdot\|:=\|\cdot\|_{0}$ are, respectively, just the $L^{2}$-inner product and the $L^{2}$-norm and $H^{0,2}(U)=L^{2}(U)$. For any compact set $K \subseteq U$, define the space $H^{j, 2}(K)$ as the completion of $C^{j}(K)$ with respect to $\|\cdot\|_{j}$. An element of $L_{\text {loc }}^{2}(U)$ which belongs to $H^{j, 2}(K)$, for all compact subdomains $K$ of $U$, is said to have strong derivatives up to order $j$. The space of all functions in $L_{\text {loc }}^{2}(U)$ having strong derivatives up to order $j$ will be denoted by $H_{\mathcal{K}}^{j, 2}(U)$.

Fix $f \in L^{2}(U), u_{0} \in H^{m, 2}(U)$ and search for (weak) solutions satisfying the generalized Dirichlet problem $\left\{\begin{array}{l}L u=f \text { on } U \\ u-u_{0} \in H_{0}^{m, 2}(U)\end{array}\right.$. If

$$
L:=\sum_{0 \leq|\alpha|,|\beta| \leq m}(-1)^{|\alpha|} a_{\alpha, \beta} D^{\alpha+\beta}, \quad \operatorname{Dom} L:=H_{\mathcal{K}}^{2 m, 2}(U) \cap H_{0}^{m, 2}(U),
$$

is a strongly elliptic operator of order $2 m$ - in [96] they are called uniformly elliptic - where the coefficients $a_{\alpha, \beta}$ all belong to $\mathbb{C},{ }^{30}$ then according to $[96$, Theorem 1 ,

\footnotetext{
${ }^{29}$ The author would like to thank professor Paulo Domingos Cordaro for showing and lending his printed copy of [96], and also for providing the second example, which gives a natural motivation for introducing the concept of strong ellipticity.

${ }^{30}$ In [96] $L$ has variable coefficients, but because of the present context, $L$ is assumed to have constant complex coefficients.
} 
page 658], the problem $L u=f$ with zero boundary condition is subject to the Fredholm alternative, a concept which will be explained in more detail: first of all, a result contained in [96, Theorem 1] states that the Dirichlet problem mentioned at the beginning - more precisely, the problem of finding $u \in H^{m, 2}(U)$ such that $u-u_{0} \in H_{0}^{m, 2}(U)$ and

$$
\sum_{0 \leq|\alpha|,|\beta| \leq m}\left\langle D^{\alpha} \phi, a_{\alpha, \beta} D^{\beta} u\right\rangle=\langle\phi, f\rangle,
$$

for all $\phi \in C_{c}^{\infty}(U)$ - with $L$ substituted by $L+k$, admits a unique solution in $H^{m, 2}(U)$ if the real number $k>0$ is chosen sufficiently large. Hence, [96, Theorem 2, page 661] guarantees that this solution actually has strong derivatives up to order $2 m$, so it also belongs to $H_{\mathcal{K}}^{2 m, 2}(U)$. Suppose $u_{0}=0$. Then, these facts, together with Gårding's inequality on page $658,{ }^{31}$ show that there exists $k_{0}>0$ such that $\left.\left(L+k_{0}\right)^{-1}\right|_{L^{2}(U)}$ is a bounded operator from $L^{2}(U)$ into $H_{\mathcal{K}}^{2 m, 2}(U) \cap H_{0}^{m, 2}(U)=$ Dom $L$. The RellichKondrachov Theorem (see [31, Theorem 9.16, page 285]) guarantees that the embed$\operatorname{ding} H^{1,2}(U) \hookrightarrow L^{2}(U)$ is compact, so the embedding $H_{0}^{m, 2}(U) \hookrightarrow L^{2}(U)$ is also compact, since the inclusion $\left(H_{0}^{m, 2}(U),\|\cdot\|_{m}\right) \hookrightarrow\left(H_{0}^{1,2}(U),\|\cdot\|_{1}\right)$ is clearly continuous. This fact may be used to prove compactness of $\left.\left(L+k_{0}\right)^{-1}\right|_{L^{2}(U)}:\left(L^{2}(U),\|\cdot\|\right) \longrightarrow$ $\left(L^{2}(U),\|\cdot\|\right)$, for if $\left\{x_{n}\right\}_{n \in \mathbb{N}}$ is a bounded sequence in $L^{2}(U)$, then $\left\{\left(L+k_{0}\right)^{-1} x_{n}\right\}_{n \in \mathbb{N}}$ is bounded in $H_{0}^{m, 2}(U)$, by continuity of $\left(L+k_{0}\right)^{-1}$. Therefore, since the inclusion $H_{0}^{m, 2}(U) \hookrightarrow L^{2}(U)$ is compact, $\left\{\left(L+k_{0}\right)^{-1} x_{n}\right\}_{n \in \mathbb{N}}$ possesses a convergent subsequence in $L^{2}(U)$, proving the desired compactness.

Finally, one may use the Fredholm alternative for compact operators to obtain information about the problem $L u=f$ with $u_{0}=0$ :

Fredholm Alternative for Compact Operators, [86, Theorem 4.32, page 100]: Let $T$ be a compact operator on a Hilbert space $\mathcal{H}$. Suppose $\lambda$ is a nonzero complex number. Then, $T-\lambda I$ is injective if, and only if, it is surjective.

Fix $\lambda_{0} \neq 0$. Then, there exist two (mutually exclusive) possibilities:

(i) $\lambda_{0}$ is an eigenvalue for $A:=\left.\left(L+k_{0}\right)^{-1}\right|_{L^{2}(U)}$, which amounts to saying that there exists $0 \neq u \in L^{2}(U)$ such that $A u=\lambda_{0} u$ (in particular, this implies that $u$ belongs to Dom $L$ ) or, equivalently, that $L u=\left(-k_{0}+\lambda_{0}^{-1}\right) u$;

(ii) $A-\lambda_{0}$ is a bijection of $L^{2}(U)$ onto $L^{2}(U)$. This last possibility is equivalent to the statement that $\left.\left(A-\lambda_{0}\right) \circ\left(L+k_{0}\right)\right|_{\operatorname{Ran} A}=I-\left.\lambda_{0}\left(L+k_{0}\right)\right|_{\operatorname{Ran} A}$ is a bijection of

\footnotetext{
${ }^{31}$ See also [109, Theorem 6.3, page 58] and [109, V.2d, page 428].
} 
Ran $A$ onto $L^{2}(U)$, which is the same as saying that $\left.L\right|_{\operatorname{Ran} A}+k_{0}-\lambda_{0}^{-1}$ is a bijection of Ran $A$ onto $L^{2}(U)$.

Now, since every complex number $\lambda$ different from $k_{0}$ may be written as $k_{0}-\lambda_{0}^{-1}$, for some $\lambda_{0} \in \mathbb{C} \backslash\{0\}$, and the case $\lambda=k_{0}$ is already known to satisfy the property that $\left(L+k_{0}\right)^{-1}: L^{2}(U) \longrightarrow \operatorname{Ran} A$ is a bijection, the following conclusion is finally attained: for any complex number $\lambda$, either the problem $(L-\lambda I) u=0$ has a nonzero solution in $H_{\mathcal{K}}^{2 m, 2}(U) \cap H_{0}^{m, 2}(U)$, or the nonhomogeneous problem $(L-\lambda I) u=f$ has a unique solution in $H_{\mathcal{K}}^{2 m, 2}(U) \cap H_{0}^{m, 2}(U)$, for every $f \in L^{2}(U)$.

2. Let $\mathbb{D}:=\{z \in \mathbb{C}:|z|<1\}$ be the unit open disk,

$$
\frac{\partial}{\partial \bar{z}}:=\frac{1}{2}\left(\frac{\partial}{\partial x}+i \frac{\partial}{\partial y}\right)
$$

the Cauchy-Riemann operator and search for functions $u \in C^{2}(\mathbb{D}) \cap C(\overline{\mathbb{D}})$ which satisfy the Dirichlet problem $\left\{\begin{array}{l}L u=0 \text { on } \mathbb{D} \\ u=0 \text { on } \partial \mathbb{D}\end{array}\right.$, where

$$
L:=\left(\frac{\partial}{\partial \bar{z}}\right)^{2}=\frac{1}{4}\left(\frac{\partial^{2}}{\partial x^{2}}+2 i \frac{\partial}{\partial x} \frac{\partial}{\partial y}+\frac{\partial^{2}}{\partial y^{2}}\right)
$$

is an elliptic operator which is not strongly elliptic. The attempt will be to search for solutions of the form $u(z)=\bar{z} g(z)+h(z)$, where both $g$ and $h$ are holomorphic on a neighborhood of $\overline{\mathbb{D}}$. In this case, $u$ clearly satisfies $L u=0$ on $\mathbb{D}$. Imposing the condition on the boundary, one finds $g(z)=-z h(z)$ on $\partial \mathbb{D}$, so define $g(z):=-z h(z)$. This shows that every function of the form $u(z)=\left(1-|z|^{2}\right) h(z)$, where $h$ is a holomorphic function on a neighborhood of $\overline{\mathbb{D}}$, is a solution for the Dirichlet problem above, so there is an infinite number of solutions for it - in other words, it is not well-posed.

Motivated by [25], the following definition will be employed:

Definition (Representation by Closed Linear Operators): In the next theorems the following notations will be used: let $\mathcal{X}$ be a complete Hausdorff locally convex space, $\mathcal{D} \subseteq \mathcal{X}$ a dense subspace, $\mathfrak{g}$ a real finite-dimensional Lie algebra and $\eta$ a function defined on $\mathfrak{g}$ assuming values on (not necessarily continuous) closed linear operators on $\mathcal{X}$ - in other words, $\eta(X)$ is a closed linear operator on $\mathcal{X}$, for all $X \in \mathfrak{g}$. Assume, also, that $\mathcal{D}$ is a core for all the operators in $\eta[\mathfrak{g}]$, so that $\mathcal{D}$ will be called a core domain for $\eta[\mathfrak{g}]$, and that $\eta_{\mathcal{D}}: \mathfrak{g} \longrightarrow \mathcal{L} \subseteq \operatorname{End}(\mathcal{D})$, defined by $\eta_{\mathcal{D}}(X):=\left.\eta(X)\right|_{\mathcal{D}}$, is a faithful Lie algebra representation onto $\mathcal{L}$. Then, the triple $(\mathcal{D}, \mathfrak{g}, \eta)$ is said to be a representation of $\mathfrak{g}$ by closed linear operators on $\mathcal{X}$. It is said to exponentiate, or to be exponentiable, 
if there exists a simply connected Lie group $G$ having $\mathfrak{g}$ as its Lie algebra and a strongly continuous locally equicontinuous representation $V: G \longrightarrow \mathcal{L}(\mathcal{X})$ such that $\mathcal{D} \subseteq C^{\infty}(V)$ and $d V(X)=\overline{\left.\eta(X)\right|_{\mathcal{D}}}=\eta(X)$, for all $X \in \mathfrak{g}$. Also, identical terminologies of Definition 2.6 will be used to indicate if the operators $d V(X), X \in \mathfrak{g}$, are generators of equicontinuous, or even $\Gamma$-isometrically equicontinuous groups.

Theorem 2.11, below, generalizes [25, Theorem 2.8] to higher-order strongly elliptic operators, but still in the Banach space context. Differently of what is done in [25], this theorem will be proved for an arbitrary dense core domain $\mathcal{D}$ which is left invariant by the operators in $\mathcal{L}$ - in [25], the claim is proved for the particular case in which the domain is

$$
\mathcal{X}_{\infty}:=\bigcap_{n=1}^{+\infty} \bigcap\left\{\operatorname{Dom}\left[\eta\left(X_{i_{1}}\right) \ldots \eta\left(X_{i_{k}}\right) \ldots \eta\left(X_{i_{n}}\right)\right]: X_{i_{k}} \in \mathcal{B}, 1 \leq k \leq n\right\}
$$

where $\mathcal{B}$ is a basis for $\mathfrak{g}$ (by what was proved at the beginning of this section, $\mathcal{X}_{\infty}$ is the maximal $\mathrm{C}^{\infty}$ domain for the set $\left.\left\{\eta\left(X_{k}\right)\right\}_{1 \leq k \leq d}\right)$. The references [25, Theorem 2.1] and [109, Theorem 2.2, page 80; Lemma 2.3, page 82] were vital sources of inspiration for the proof of Theorem 2.11. ${ }^{32}$ The theorems of the latter reference obtain some estimates which are fulfilled by strongly elliptic linear operators coming from a strongly continuous representation of a given Lie group and, together with Theorem 5.1 of this same book (page 30), prove (in particular) the following:

Let $\mathcal{X}$ be a Banach space, $G$ a Lie group with Lie algebra $\mathfrak{g}, V: G \longrightarrow \mathcal{L}(\mathcal{X})$ a strongly continuous representation of $G$ by bounded operators and $H_{m} \in \mathfrak{U}(\partial V[\mathfrak{g}])_{\mathbb{C}}$ an element of order $m$ which is strongly elliptic. Then, $-H_{m}$ is an infinitesimal pregenerator of a strongly continuous semigroup $t \longmapsto S(t)$ satisfying $S(t)[\mathcal{X}] \subseteq C^{\infty}(V)$ for $t \in(0,1]$ and, for each $n \in \mathbb{N}$, there exists a constant $C_{n}>0$ such that

$$
\rho_{n}(S(t) y) \leq C_{n} t^{-\frac{n}{m}}\|y\|, \quad t \in(0,1], y \in \mathcal{X} .
$$

More precisely, such constants are of the form $C_{n}=K L^{n} n$ !, for some $K, L \geq 1 .^{33}$

To be more precise, [109, Theorem 5.1, page 30] is stated in the form of weakly continuous semigroups or, in other words, it states that the weak closure of $-H_{m}$ (the weak

\footnotetext{
${ }^{32}$ See also [80] and [81].

${ }^{33}$ In order to avoid unnecessary confusions, it should be noted that references [25], [26] and [109] (see page 30) use a slightly different convention from the one employed in this manuscript to define infinitesimal generators: if an operator $H_{m}$ is strongly elliptic then, according to their definitions, $\overline{H_{m}}$ will be the generator of a one-parameter semigroup. However, using the definition of infinitesimal generators of this thesis, if $H_{m}$ is strongly elliptic, then $-\overline{H_{m}}$ will be the generator of a one-parameter group. Therefore, for example, $-\sum_{k=1}^{d}\left[\partial V\left(X_{k}\right)\right]^{2}$ is a strongly elliptic operator and $\overline{\sum_{k=1}^{d}\left[\partial V\left(X_{k}\right)\right]^{2}}$ generates a one-parameter semigroup, with the definitions employed here.
} 
closure of an operator is defined in an analogous way of the usual closure, but with the usual topology of the Banach space $\mathcal{X}$ substituted by the weak topology) generates a weakly continuous semigroup $t \longmapsto S(t)$, which means $f(S(t) x)$ converges to $f\left(S\left(t_{0}\right) x\right)$, whenever $t_{0} \in[0,+\infty), f \in \mathcal{X}^{\prime}$ and $x \in \mathcal{X}$. However, this implies $t \longmapsto S(t)$ is strongly continuous (by [30, Corollary 3.1.8, page 168], a weakly continuous one-parameter semigroup on a Banach space is strongly continuous, and its weak and strong generators coincide), $-H_{m}$ is closable with respect to the norm topology, and its closure is the generator of $t \longmapsto S(t)$, in the strong sense, by a simple argument: denote by $\tilde{H}_{m}$ the generator of $t \longmapsto S(t)$, in the strong sense, and let $C^{\infty}(V)$ be the space of smooth vectors with respect to the strongly continuous semigroup $t \longmapsto S(t)$; by [109, Theorem 5.1, page 30], the weak closure $-\overline{H_{m}} w$ of $-H_{m}$ is the generator of $t \longmapsto S(t)$, in the weak sense, so $\tilde{H}_{m}=-\bar{H}_{m} w$, due to [30, Corollary 3.1.8, page 168]. Hence, $-\bar{H}_{m} \subset \tilde{H}_{m}=-{\overline{H_{m}}}^{w}$, because $\tilde{H}_{m}$ is a closed operator, with respect to the norm topology. Since $S$ leaves the dense subspace $C^{\infty}(V)$ invariant, it follows that it is a core for $\tilde{H}_{m}$, by Lemma 1.2.4.3, which proves

$$
\tilde{H}_{m}=\overline{\left.\tilde{H}_{m}\right|_{C^{\infty}(V)}}=-\overline{\left.{\overline{H_{m}}}^{w}\right|_{C^{\infty}(V)}}=-\overline{H_{m}},
$$

where in the last equality it was used that Dom $H_{m}:=C^{\infty}(V)$.

Regarding the reciprocal question or, in other words, the issue of exponentiability, one has the following result:

Theorem 2.11 (Exponentiation - Banach Space Version, Isometric Case): Let $\mathcal{X}$ be a Banach space, $\mathfrak{g}$ a real finite-dimensional Lie algebra with an ordered basis $\left(X_{k}\right)_{1 \leq k \leq d},(\mathcal{D}, \mathfrak{g}, \eta)$ a representation of $\mathfrak{g}$ by closed linear operators on $\mathcal{X}$, where $B_{k}:=\eta\left(X_{k}\right), 1 \leq k \leq d$, and $H_{m}$ an element of order $m \geq 2$ of $\mathfrak{U}\left(\eta_{\mathcal{D}}[\mathfrak{g}]\right)_{\mathbb{C}}$, where $\mathfrak{U}\left(\eta_{\mathcal{D}}[\mathfrak{g}]\right)_{\mathbb{C}}:=\mathfrak{U}\left(\eta_{\mathcal{D}}[\mathfrak{g}]\right)+i \mathfrak{U}\left(\eta_{\mathcal{D}}[\mathfrak{g}]\right)$ is the complexification of $\mathfrak{U}\left(\eta_{\mathcal{D}}[\mathfrak{g}]\right)$. Suppose that the following hypotheses are valid:

1. $\eta(X)$ is a conservative operator, for every $X \in \mathfrak{g}$;

2. $-H_{m}$ is an infinitesimal pregenerator of a strongly continuous semigroup $t \longmapsto S(t)$ satisfying $S(t)[\mathcal{X}] \subseteq \mathcal{D}$, for each $t>0$ and, for each $n \in \mathbb{N}$ satisfying $0<n \leq m-1$, there exists $C_{n}>0$ for which the estimates

$$
\rho_{n}(S(t) y) \leq C_{n} t^{-\frac{n}{m}}\|y\|
$$

are verified for all $t \in(0,1]$ and all $y \in \mathcal{X}$, where $B_{0}:=I$ and

$$
\rho_{n}(x):=\max \left\{\left\|B_{i_{1}} \ldots B_{i_{k}} \ldots B_{i_{n}} x\right\|: 1 \leq k \leq n, 0 \leq i_{k} \leq d\right\}
$$

for all $x \in \mathcal{D}$. 
Then, $(\mathcal{D}, \mathfrak{g}, \eta)$ exponentiates to a representation by isometries: in other words, each $\eta(X), X \in \mathfrak{g}$, is the generator of a group of isometries.

Proof of Theorem 2.11: The first task of this proof is to obtain the estimates

$$
\rho_{n}(y) \leq \epsilon^{m-n}\left\|H_{m}(y)\right\|+\frac{E_{n}}{\epsilon^{n}}\|y\|, \quad y \in \mathcal{D}
$$

valid for all $0<\epsilon \leq 1,0<n \leq m-1$ and some $E_{n}>0$.

The semigroup $t \longmapsto S(t)$ is strongly continuous, so there exist constants $M \geq 1$ and $w \geq 0$ such that

$$
\|S(t) y\| \leq M \exp (w t)\|y\|
$$

for all $y \in \mathcal{X}$ and $t \geq 0$. Now, if $0<\delta<1$ satisfies $0 \leq w<\frac{1}{\delta}$, the resolvent $\mathrm{R}\left(1,-\delta \overline{H_{m}}\right)=$ $\left(1+\delta \overline{H_{m}}\right)^{-1}$ is well-defined and

$$
\mathrm{R}\left(1,-\delta \overline{H_{m}}\right)=\int_{0}^{+\infty} \exp (-t) S_{\delta t}(y) d t, \quad y \in \mathcal{X}
$$

with

$$
\int_{0}^{+\infty} \exp (-t)\left\|S_{\delta t}(y)\right\| d t<\infty
$$

Define $B_{k}:=\eta\left(X_{k}\right)$, for $1 \leq k \leq d$, and fix a monomial $B^{\mathrm{u}}$ of size $0<n \leq m-1$ in the operators $\left\{B_{k}\right\}_{1 \leq k \leq d}$. Fix $t>0$. If $\delta t \leq 1$, then

$$
\left\|B^{\mathrm{u}} S_{\delta t}(y)\right\| \leq C_{n}(\delta t)^{-\frac{n}{m}}\|y\|, \quad y \in \mathcal{X}
$$

by $(2.11 .1)$; if $\delta t>1$, then

$$
\left\|B^{\mathrm{u}} S_{\delta t}(y)\right\|=\left\|B^{\mathrm{u}} S_{1+(\delta t-1)}\right\| \leq C_{n} M \exp (w(\delta t-1))\|y\|, \quad y \in \mathcal{X} .
$$

These inequalities and

$$
\frac{1}{\exp (t)} \leq \sqrt[m]{\frac{1}{\exp (t)}} \leq t^{-\frac{k}{m}} \sqrt[m]{k !}
$$

with $k=m-n$ and $k=n$, respectively, allow one to obtain, for every $0<\delta<1 /(2 w)$, the estimates

$$
\begin{gathered}
\int_{0}^{+\infty} \exp (-t)\left\|B^{\mathrm{u}} S_{\delta t}(y)\right\| d t \\
\leq C_{n}\|y\|\left(\int_{0}^{1 / \delta} \frac{\exp (-t)}{(\delta t)^{\frac{n}{m}}} d t+M \exp (-w) \int_{1 / \delta}^{+\infty} \exp ((w \delta-1) t) d t\right)
\end{gathered}
$$




$$
\begin{gathered}
=C_{n}\|y\|\left(\left.\delta^{-\frac{n}{m}}\left[\exp (-t) \frac{m}{m-n} t^{\frac{m-n}{m}}\right]\right|_{t=0} ^{t=1 / \delta}+\delta^{-\frac{n}{m}} \frac{m}{m-n} \int_{0}^{1 / \delta} \exp (-t) t^{\frac{m-n}{m}} d t\right. \\
\left.+M \exp (-w) \frac{\exp (w-1 / \delta)}{1-w \delta}\right) \\
\leq C_{n}\|y\|\left(\delta^{-\frac{n}{m}} \delta^{\frac{m-n}{m}} \sqrt[m]{(m-n) !} \frac{m}{m-n} \delta^{\frac{n-m}{m}}\right. \\
\left.+\delta^{-\frac{n}{m}} \frac{m}{m-n} \int_{0}^{+\infty} \exp (-t) t^{\frac{m-n}{m}} d t+2 M \delta^{\frac{n}{m}} \sqrt[m]{n !}\right) \\
\leq C_{n}\left(\sqrt[m]{(m-n) !} \frac{m}{m-n}+I_{0} \frac{m}{m-n}+2 M \sqrt[m]{n !}\right) \delta^{-\frac{n}{m}}\|y\|,
\end{gathered}
$$

for all $y \in \mathcal{X}$, where

$$
I_{0}:=\int_{0}^{+\infty} \exp (-t) t^{\frac{m-n}{m}} d t
$$

(in the last inequality it was used that $\delta^{-\frac{n}{m}}>\delta^{\frac{n}{m}}$ ). Therefore, if $y$ is any vector in $\mathcal{X}$, then

$$
\int_{0}^{+\infty} \exp (-t)\left\|B^{\mathrm{u}} S_{\delta t}(y)\right\| d t<\infty
$$

and, in particular, the integral $\int_{0}^{+\infty} \exp (-t) B^{\mathrm{u}} S_{\delta t}(y) d t$ defines an element in $\mathcal{X}$. By closedness of each operator $B_{k}$ it follows that $\left(1+\delta \overline{H_{m}}\right)^{-1}(y) \in \operatorname{Dom} B^{\mathrm{u}}$ and

$$
B^{\mathrm{u}}\left(1+\delta \overline{H_{m}}\right)^{-1}(y)=\int_{0}^{+\infty} \exp (-t) B^{\mathrm{u}} S_{\delta t}(y) d t
$$

Also, the estimates above show that there exists $E_{n}^{\prime}>0$ independent of $\delta$ such that

$$
\left\|B^{\mathrm{u}}\left(1+\delta \overline{H_{m}}\right)^{-1}(y)\right\| \leq E_{n}^{\prime} \delta^{-\frac{n}{m}}\|y\|, \quad y \in \mathcal{X} .
$$

Hence, if $y$ is of the form $y=\left(1+\delta \overline{H_{m}}\right)(w)=\left(1+\delta H_{m}\right)(w), w \in \mathcal{D}$, one obtains

$$
\begin{gathered}
\left\|B^{\mathrm{u}}(w)\right\|=\left\|B^{\mathrm{u}}\left(1+\delta \overline{H_{m}}\right)^{-1}\left[\left(1+\delta \overline{H_{m}}\right)(w)\right]\right\| \leq E_{n}^{\prime} \delta^{-\frac{n}{m}}\left\|\left(1+\delta \overline{H_{m}}\right)(w)\right\| \\
\leq E_{n}^{\prime} \delta^{\frac{m-n}{m}}\left\|H_{m}(w)\right\|+E_{n}^{\prime} \delta^{-\frac{n}{m}}\|w\| .
\end{gathered}
$$

Now, define $\epsilon_{n}(\delta):=\left(E_{n}^{\prime}\right)^{\frac{1}{m-n}} \delta^{\frac{1}{m}}$. If $\epsilon_{n}\left(\delta_{0}\right)>1$, for some $\delta_{0}$, then (2.11.2) is proved since, by continuity, the range of the function $\epsilon_{n}$ must contain the interval $(0,1]$ when $\delta$ runs through $(0,1 /(2 w))$, as a consequence of Weierstrass Intermediate Value Theorem. Then, defining $E_{n}:=\left(E_{n}^{\prime}\right)^{\frac{m}{m-n}}$, one obtains

$$
E_{n} \epsilon_{n}(\delta)^{-n}=\left(E_{n}^{\prime}\right)^{\frac{m}{m-n}} \epsilon_{n}(\delta)^{-n}=E_{n}^{\prime} \delta^{-\frac{n}{m}}
$$


SO

$$
\rho_{n}(y) \leq \epsilon^{m-n}\left\|H_{m}(y)\right\|+\frac{E_{n}}{\epsilon^{n}}\|y\|, \quad y \in \mathcal{D}, 0<\epsilon \leq 1,
$$

as desired. However, if $\epsilon_{n}(\delta)<1$, for all $\delta \in(0,1 /(2 w))$, then multiply the function $\epsilon_{n}$ by a real constant $a$ greater than 1 so that the range of $\epsilon_{n}^{\prime}:=a \epsilon_{n}$ contains 1 . This reduces the problem to the previous case and finishes the first step of the proof.

The idea, now, is to divide the rest of the proof into three items:

- Given $q \in \mathbb{N}, q \geq 1$, there exists a strictly positive constant $K_{q}$ such that

$$
\rho_{n}(S(t) y) \leq K_{q} \sup _{0 \leq j \leq q}\left\|H_{m}^{j}(y)\right\|
$$

for all $y \in \mathcal{D},(q-1)(m-1)<n \leq q(m-1)$ and $t \in(0,1]$. This implies, in particular, that given $q \in \mathbb{N}, q \geq 1$, and $y \in \mathcal{D}$, there exists $K_{y, q}>0$ satisfying $\rho_{n}(S(t) y) \leq K_{y, q}$, for all $t \in(0,1]$ and $(q-1)(m-1)<n \leq q(m-1)$ - actually, this is the result that will be invoked, later:

The procedure is by induction on $q$. By what was proved earlier, to each fixed $0<n \leq m-1$ the estimates

$$
\rho_{n}(y) \leq \epsilon^{m-n}\left\|H_{m}(y)\right\|+\frac{E_{n}}{\epsilon^{n}}\|y\|, \quad y \in \mathcal{D},
$$

are valid for some $E_{n}>0$ and all $0<\epsilon \leq 1$. This shows the existence of a $K_{1}>0$ satisfying

$$
\rho_{n}(S(t) y) \leq K_{1} \sup _{0 \leq j \leq 1}\left\|H_{m}^{j}(y)\right\|, \quad y \in \mathcal{D}, 0<n \leq m-1, t \in(0,1],
$$

since $H_{m} S(t)=S(t) H_{m}$ on Dom $H_{m}$ and $\|S(t)\| \leq M e^{w t}$, for some $M \geq 1, w \geq 0$ and all $t \geq 0$. Now, suppose that, for some fixed $q \in \mathbb{N}, q \geq 1$, the inequality

$$
\rho_{n}(S(t) y) \leq K_{q} \sup _{0 \leq j \leq q}\left\|H_{m}^{j}(y)\right\|
$$

is true, for all $y \in \mathcal{D},(q-1)(m-1)<n \leq q(m-1), t \in(0,1]$. Fix $y \in \mathcal{D}$. A monomial $B^{\mathrm{u}}$ of size $q(m-1)<n \leq(q+1)(m-1)$ in the operators $\left(B_{k}\right)_{1 \leq k \leq d}$ may be decomposed as $B^{\mathbf{u}}=B^{\mathbf{u}_{0}} B^{\mathbf{u}^{\prime}}$, with $\left|\mathbf{u}_{0}\right|=m-1$ and $\left|\mathbf{u}^{\prime}\right|=n-(m-1)$. Using (2.11.2), one obtains

$$
\left\|B^{\mathrm{u}} S(t) y\right\|=\left\|B^{\mathrm{u}_{0}} B^{\mathrm{u}^{\prime}} S(t) y\right\| \leq \epsilon^{m-(m-1)}\left\|H_{m} B^{\mathrm{u}^{\prime}} S(t) y\right\|+\frac{E_{m-1}}{\epsilon^{m-1}}\left\|B^{\mathrm{u}^{\prime}} S(t) y\right\|
$$




$$
=\epsilon\left\|H_{m} B^{\mathrm{u}^{\prime}} S(t) y\right\|+\frac{E_{m-1}}{\epsilon^{m-1}}\left\|B^{\mathrm{u}^{\prime}} S(t) y\right\|, \quad 0<t \leq 1,0<\epsilon \leq 1 .
$$

On the other hand, by the induction hypothesis,

$$
\begin{gathered}
\left\|H_{m} B^{\mathrm{u}^{\prime}} S(t) y\right\| \leq\left\|\left[\operatorname{ad}\left(H_{m}\right)\left(B^{\mathrm{u}^{\prime}}\right)\right] S(t) y\right\|+\left\|B^{\mathrm{u}^{\prime}} H_{m} S(t) y\right\| \\
\leq k(n-(m-1)) m \rho_{n}(S(t) y)+K_{q} \sup _{0 \leq j \leq q}\left\|H_{m}^{j} H_{m}(y)\right\|, \quad 0<t \leq 1,
\end{gathered}
$$

$k$ being a constant depending only on $d, H_{m}$ and on the numbers $c_{i j}$ which are defined by $\left[B_{i}, B_{j}\right]=\sum_{k=1}^{d} c_{i j}^{(k)} B_{k}$, for all $1 \leq i \neq j \leq d$ (see Section 1.5). Hence, using the induction hypothesis again, one obtains

$$
\begin{gathered}
\left\|B^{\mathrm{u}} S(t) y\right\| \leq \epsilon\left[k(n-(m-1)) m \rho_{n}(S(t) y)+K_{q} \sup _{0 \leq j \leq q+1}\left\|H_{m}^{j}(y)\right\|\right] \\
+\frac{E_{m-1}}{\epsilon^{m-1}} K_{q} \sup _{0 \leq j \leq q}\left\|H_{m}^{j}(y)\right\|, \quad 0<t \leq 1,0<\epsilon \leq 1 .
\end{gathered}
$$

Choosing an $1 \geq \epsilon_{0}>0$ such that $\epsilon_{0} k(n-(m-1)) m<1$, and taking the maximum over all monomials $B^{\mathrm{u}}$ of size $n$ gives

$$
\begin{gathered}
\rho_{n}(S(t) y) \leq \frac{\epsilon_{0} K_{q} \sup _{0 \leq j \leq q+1}\left\|H_{m}^{j}(y)\right\|+\frac{E_{m-1}}{\epsilon_{0}^{m-1}} K_{q} \sup _{0 \leq j \leq q}\left\|H_{m}^{j}(y)\right\|}{1-\epsilon_{0} k(n-(m-1))} \\
\quad \leq\left[\frac{K_{q}\left(\epsilon_{0}+\frac{E_{m-1}}{\epsilon_{0}^{m-1}}\right)}{1-\epsilon_{0} k(n-(m-1))}\right] \sup _{0 \leq j \leq q+1}\left\|H_{m}^{j}(y)\right\|, \quad 0<t \leq 1 .
\end{gathered}
$$

This concludes the induction proof.

- There exist $K, L \geq 1$ such that, given $q \in \mathbb{N}, q \geq 1$ and $(q-1)(m-1)<n \leq q(m-1)$, there exists a strictly positive constant $C_{n}$ defined by $C_{n}:=K L^{n} n$ ! satisfying

$$
\rho_{n}(S(t) y) \leq C_{n} t^{-\frac{n}{m}}\|y\|, \quad y \in \mathcal{X}, t \in(0,1] .
$$

More precisely, there exist $K, L \geq 1$ such that

$$
\rho_{n}(S(t) y) \leq K L^{n} n ! t^{-\frac{n}{m}}\|y\|,
$$

for all $n \in \mathbb{N}, n \geq 1, y \in \mathcal{X}$ and $t \in(0,1]$ :

The idea is, again, to proceed by induction. The case $q=1$ follows from (2.11.1), with $K:=D_{1}:=\max \left\{1, \max \left\{C_{j}: 0<j \leq m-1\right\}\right\}$ and $L:=1$, so it is already 
known to be true. Fix $q \geq 1$ and suppose that there exists, for every $p \leq q$ and $l \in \mathbb{N}$ satisfying $(p-1)(m-1)<l \leq p(m-1)$, a strictly positive constant $C_{l}$ (not necessarily of the form $\left.K L^{l} l !, K, L \geq 1\right)$ such that

$$
\rho_{l}(S(t) y) \leq C_{l} t^{-\frac{l}{m}}\|y\|, \quad y \in \mathcal{X}, t \in(0,1] .
$$

Fix $n \in \mathbb{N}$ satisfying $q(m-1)<n \leq(q+1)(m-1)$ (in particular, $n>m-1$ ), $0<t \leq 1$ and $\boldsymbol{y} \in \mathcal{D}$. As in the previous item, take a monomial $B^{\mathrm{u}}$ of size $q(m-1)<n \leq(q+1)(m-1)$ in the operators $\left(B_{k}\right)_{1 \leq k \leq d}$ and decompose it as $B^{\mathrm{u}}=B^{\mathrm{u}_{0}} B^{\mathrm{u}^{\prime}}$, with $\left|\mathrm{u}_{0}\right|=m-1$ and $\left|\mathbf{u}^{\prime}\right|=n-(m-1)$. Then, by Lemma 1.5.1,

$$
\begin{aligned}
& B^{\mathrm{u}} S(t) y=B^{\mathrm{u}_{0}} S_{s} B^{\mathrm{u}^{\prime}} S_{t-s}(y)+B^{\mathrm{u}_{0}}\left[\left(\operatorname{ad} B^{\mathrm{u}^{\prime}}\right)\left(S_{s}\right)\right] S_{t-s}(y) \\
& =B^{\mathrm{u}_{0}} S_{s} B^{\mathrm{u}^{\prime}} S_{t-s}(y)-\int_{0}^{s} B^{\mathrm{u}_{0}} S_{r}\left[\left(\operatorname{ad} B^{\mathrm{u}^{\prime}}\right)\left(H_{m}\right)\right] S_{t-r}(y) d r
\end{aligned}
$$

for all $0<s<t$. Like before, one has the inequality

$$
\left\|\left[\left(\operatorname{ad} B^{\mathbf{u}^{\prime}}\right)\left(H_{m}\right)\right] S_{t-r}(y)\right\| \leq k(n-(m-1)) m \rho_{n}\left(S_{t-r}(y)\right)
$$

where $k$, again, depends only on $d, H_{m}$ and on the numbers $c_{i j}$ defined by $\left[B_{i}, B_{j}\right]=$ $\sum_{j=1}^{d} c_{i j} B_{j}$, for all $1 \leq i \neq j \leq d$. Applying this inequality, together with the induction hypothesis for $q=1(n=m-1)$, one obtains from (2.11.3) the estimates

$$
\begin{gathered}
\left\|B^{\mathrm{u}} S(t) y\right\| \leq C_{m-1} s^{-\frac{m-1}{m}}\left\|B^{\mathrm{u}^{\prime}} S_{t-s}(y)\right\| \\
+\int_{0}^{s} C_{m-1} r^{-\frac{m-1}{m}}\left\|\left[\operatorname{ad}\left(B^{\mathrm{u}^{\prime}}\right)\left(H_{m}\right)\right] S_{t-r}(y)\right\| d r \\
\leq C_{m-1} s^{-\frac{m-1}{m}} C_{n-(m-1)}(t-s)^{-\frac{n-(m-1)}{m}}\|y\| \\
+C_{m-1} k(n-(m-1)) m \int_{0}^{s} r^{-\frac{m-1}{m}} \rho_{n}\left(S_{t-r}(y)\right) d r .
\end{gathered}
$$

Making the changes of variables $s=\lambda t$, where $\lambda \in(0,1)$, and $r=u t$, inside the integral, such inequality becomes

$$
\begin{aligned}
& \left\|B^{\mathrm{u}} S(t) y\right\| \leq C_{m-1} C_{n-(m-1)} t^{-\frac{n}{m}} \lambda^{-\frac{m-1}{m}}(1-\lambda)^{-\frac{n-(m-1)}{m}}\|y\| \\
& +C_{m-1} k(n-(m-1)) m t^{\frac{1}{m}} \int_{0}^{\lambda} u^{-\frac{m-1}{m}} \rho_{n}\left(S_{t(1-u)}(y)\right) d u .
\end{aligned}
$$

Putting $\lambda=n^{-m}$, one has that $\lambda^{-\frac{m-1}{m}}=n^{m-1}$ and

$$
(1-\lambda)^{-\frac{n-(m-1)}{m}}=(1-\lambda)^{-\frac{n}{m}}(1-\lambda)^{\frac{m-1}{m}} \leq(1-\lambda)^{-\frac{n}{m}} \leq c_{m},
$$


where

$$
c_{m}:=\sup _{n \geq m}\left\{\left(1-n^{-m}\right)^{-\frac{n}{m}}\right\}>1 .
$$

Hence, taking the maximum over all the monomials of size $n$, the last inequality becomes

$$
\begin{gathered}
\rho_{n}(S(t) y) \leq C_{m-1} C_{n-(m-1)} t^{-\frac{n}{m}} \lambda^{-\frac{m-1}{m}}(1-\lambda)^{-\frac{n-(m-1)}{m}}\|y\| \\
+C_{m-1} k(n-(m-1)) m t^{\frac{1}{m}} \int_{0}^{\lambda} u^{-\frac{m-1}{m}} \rho_{n}\left(S_{t(1-u)}(y)\right) d u \\
\leq C_{m-1} C_{n-(m-1)} t^{-\frac{n}{m}} n^{m-1} c_{m}\|y\| \\
+C_{m-1} k(n-(m-1)) m t^{\frac{1}{m}} \int_{0}^{n^{-m}} u^{-\frac{m-1}{m}} \rho_{n}\left(S_{t(1-u)}(y)\right) d u .
\end{gathered}
$$

But, since the $0<t \leq 1$ fixed was arbitrary, one may repeat this procedure and iterate $j-1$ times the last inequality to obtain

$$
\rho_{n}(S(t) y) \leq C_{m-1} C_{n-(m-1)} t^{-\frac{n}{m}} n^{m-1} c_{m}\|y\| \sum_{i=0}^{j-1} a_{i}+R_{j},
$$

where $a_{0}:=1$,

$$
\begin{gathered}
a_{i}:=\left(C_{m-1} k(n-(m-1)) m t^{\frac{1}{m}}\right)^{i} \int_{0}^{n^{-m}} u_{1}^{-\frac{m-1}{m}}\left(1-u_{1}\right)^{-\frac{n}{m}} d u_{1} \ldots \\
\ldots \int_{0}^{n^{-m}} u_{i}^{-\frac{m-1}{m}}\left(1-u_{i}\right)^{-\frac{n}{m}} d u_{i} \\
\leq\left(C_{m-1} k n m t^{\frac{1}{m}}\right)^{i}\left[\sup _{n \geq m}\left(1-n^{-m}\right)^{-\frac{n}{m}}\right]^{i}\left(\int_{0}^{n^{-m}} u^{-\frac{m-1}{m}} d u\right)^{i} \\
=\left(C_{m-1} k n m c_{m} t^{\frac{1}{m}}\right)^{i}\left(m n^{-1}\right)^{i}=\left(C_{m-1} k m^{2} c_{m} t^{\frac{1}{m}}\right)^{i},
\end{gathered}
$$

if $i \geq 1$, and

$$
\begin{gathered}
R_{j}:=\left(C_{m-1} k(n-(m-1)) m t^{\frac{1}{m}}\right)^{j} \int_{0}^{n^{-m}} u_{1}^{-\frac{m-1}{m}} \ldots \\
\ldots \int_{0}^{n^{-m}} u_{j}^{-\frac{m-1}{m}} \rho_{n}\left(S_{t\left(1-u_{1}\right) \ldots\left(1-u_{j}\right)}(y)\right) d u_{1} \ldots d u_{j},
\end{gathered}
$$

for all $j \geq 1$. Therefore, by the previous item,

$$
R_{j} \leq\left(C_{m-1} k m^{2} t^{\frac{1}{m}}\right)^{j} K_{y, q+1}
$$


If $t_{m}>0$ is chosen so that $C_{m-1} k m^{2} c_{m}\left(t_{m}\right)^{\frac{1}{m}}=\frac{1}{2}$, that is, if

$$
t_{m}=\left(2 C_{m-1} k m^{2} c_{m}\right)^{-m},
$$

then for all $0<t \leq t_{m}$,

$$
\sum_{k=0}^{+\infty} a_{k} \leq \sum_{k=0}^{+\infty}\left(C_{m-1} k m^{2} c_{m} t^{\frac{1}{m}}\right)^{k} \leq \sum_{k=0}^{+\infty}\left(C_{m-1} k m^{2} c_{m}\left(t_{m}\right)^{\frac{1}{m}}\right)^{k}=\sum_{k=0}^{+\infty}\left(\frac{1}{2}\right)^{k}=2
$$

and

$$
\begin{gathered}
\lim _{k \rightarrow+\infty} R_{k} \leq{ }^{34} K_{y, q+1}\left[\lim _{k \rightarrow+\infty}\left(C_{m-1} k m^{2} c_{m} t^{\frac{1}{m}}\right)^{k}\right] \\
\leq K_{y, q+1}\left[\lim _{k \rightarrow+\infty}\left(C_{m-1} k m^{2} c_{m}\left(t_{m}\right)^{\frac{1}{m}}\right)^{k}\right]=K_{y, q+1}\left[\lim _{k \rightarrow+\infty}\left(\frac{1}{2}\right)^{k}\right]=0,
\end{gathered}
$$

showing that $\lim _{k \rightarrow+\infty} R_{k}=0$. Hence, taking the limit $j \rightarrow+\infty$ in (2.11.4), it follows that

$$
\rho_{n}(S(t) y) \leq 2 C_{m-1} C_{n-(m-1)} n^{m-1} c_{m} t^{-\frac{n}{m}}\|y\|,
$$

if $t \in\left(0, t_{m}\right]$ and $y \in \mathcal{D}$. A quick induction on this formula ${ }^{35}$ gives

$$
\rho_{n}(S(t) y) \leq D_{1}\left[2 D_{1} c_{m}\right]^{q} n^{q(m-1)} t^{-\frac{n}{m}}\|y\|, \quad t \in\left(0, t_{m}\right], y \in \mathcal{D}
$$

where, again, $D_{1}:=\max \left\{1, \max \left\{C_{j}: 0<j \leq m-1\right\}\right\}$. Consequently,

$$
\rho_{n}(S(t) y) \leq D_{1}\left[2 e D_{1} c_{m}\right]^{n} n ! t^{-\frac{n}{m}}\|y\|, \quad t \in\left(0, t_{m}\right], y \in \mathcal{D},
$$

where it was used the estimate $n^{q(m-1)} \leq e^{n}[q(m-1)]$ !. Therefore, defining $K_{0}:=D_{1}$ and $L_{0}:=2 e D_{1} c_{m}$, the constants $C_{l}$, with $p(m-1)<l \leq(p+1)(m-1)$ and $0<p \leq q$, may be chosen as $C_{l}=K_{0} L_{0}^{l} l$ !.

The last task is to extend the inequality just obtained to all $y \in \mathcal{X}$ and to $t \in\left(t_{m}, 1\right]$. Since $S(t)[\mathcal{X}] \subseteq \mathcal{D}$ for all $t>0$ one has that, for fixed $t_{m} \geq t>\epsilon>0$ and for every $y \in \mathcal{X}$,

$$
\rho_{n}(S(t) y)=\inf _{0<\epsilon<t} \rho_{n}\left(S_{t-\epsilon} S_{\epsilon}(y)\right) \leq K_{0} L_{0}^{n} n !\left[\inf _{0<\epsilon<t}\left[(t-\epsilon)^{-\frac{n}{m}}\left\|S_{\epsilon}(y)\right\|\right]\right]
$$

\footnotetext{
${ }^{34}$ Here, it was used that $c_{m}>1$.

${ }^{35}$ More precisely, an induction proof on $q \geq 1$, on formula

$$
\rho_{n}(S(t) y) \leq 2 C_{m-1} C_{n-(m-1)} n^{m-1} c_{m} t^{-\frac{n}{m}}\|y\|, \quad(q-1)(m-1)<n \leq q(m-1)
$$
}

subjected to the restrictions $y \in \mathcal{D}$ and $t \in\left(0, t_{m}\right]$. The base case follows easily from (2.11.1) and from $D_{1} \geq 1$. The inductive step follows from a repetition of the argument done so far, in the present item, with $C_{l}$ substituted by $2 C_{m-1} C_{l-(m-1)} l^{m-1} c_{m}$. 


$$
\leq K_{0} L_{0}^{n} n !\left[\inf _{0<\epsilon<t}\left[(t-\epsilon)^{-\frac{n}{m}} M e^{w \epsilon}\right]\right]\|y\|=\left(M K_{0}\right) L_{0}^{n} n ! t^{-\frac{n}{m}}\|y\| .
$$

If $t_{m} \geq 1$, the induction proof is complete. So suppose $0<t_{m}<1$. Then, if $1 \geq t>t_{m}$ and $y \in \mathcal{X}$

$$
\begin{gathered}
\rho_{n}(S(t) y)=\rho_{n}\left(S_{t_{m}} S_{t-t_{m}}(y)\right) \leq K_{0} L_{0}^{n} n !\left(t_{m}\right)^{-\frac{n}{m}}\left\|S_{t-t_{m}}(y)\right\| \\
\leq K_{0} L_{0}^{n} n !\left(t_{m}\right)^{-\frac{n}{m}} M e^{w\left(t-t_{m}\right)}\|y\| \leq\left(M e^{w} K_{0}\right)\left(t_{m}^{-\frac{1}{m}} L_{0}\right)^{n} n ! t^{-\frac{n}{m}}\|y\|,
\end{gathered}
$$

where in the last inequality it was used that $t^{-\frac{n}{m}} \geq 1$ and that $t-t_{m}<1$. Hence, with the definitions $K:=M e^{w} K_{0}$ and $L:=t_{m}^{-\frac{1}{m}} L_{0}$ the desired inequality

$$
\rho_{n}(S(t) y) \leq K L^{n} n ! t^{-\frac{n}{m}}\|y\|,
$$

for all $y \in \mathcal{X}$ and $t \in(0,1]$ is finally obtained, concluding the induction step.

It is therefore proved that, with the definitions $K:=M e^{w} D_{1}$ and $L:=2 e M D_{1} c_{m} t_{m}^{-\frac{1}{m}}$, it is true that

$$
\rho_{n}(S(t) y) \leq K L^{n} n ! t^{-\frac{n}{m}}\|y\|,
$$

for all $n \in \mathbb{N}, n \geq 1, x \in \mathcal{X}$ and $t \in(0,1]$.

- Define $\mathcal{X}_{0}:=\bigcup_{0<t \leq 1} S(t)[\mathcal{X}]$ and

$$
C^{\omega}(\eta):=\left\{x \in \mathcal{X}_{\infty}: \sum_{n=0}^{+\infty} \frac{\rho_{n}(x)}{n !} s^{n}<\infty \text {, for some } s>0\right\} .
$$

Since $S$ is strongly continuous, $\mathcal{X}_{0}$ is dense in $\mathcal{X}$. Besides, the estimates from the previous item show that

$$
\mathcal{X}_{0} \subseteq C^{\omega}(\eta) \cap \mathcal{D}=: \mathcal{D}^{\omega}:
$$

fix $0<t \leq 1$ and $y \in \mathcal{X}$; if one chooses $r>0$ so that

$$
r<\frac{1}{L t^{-\frac{1}{m}}}
$$

then the inequality

$$
\sum_{n=0}^{+\infty} \frac{\rho_{n}(S(t) y)}{n !} r^{n} \leq K\|y\| \sum_{n=0}^{+\infty} L^{n} t^{-\frac{n}{m}} r^{n}<\infty
$$

proves the inclusion. Therefore, $\mathcal{D}^{\omega}$ is dense in $\mathcal{X}$. Now, because the inclusion $C^{\omega}(\eta) \subseteq C^{\omega}\left(B_{k}\right)$ is valid for every $1 \leq k \leq d$ and each $B_{k}$ is conservative, it follows 
that every $\left.B_{k}\right|_{\mathcal{D}^{\omega}}$ is an infinitesimal pregenerator of a strongly continuous one parameter group of isometries, by [111, Theorem 1] - see Section 1.4. Hence, since $\mathcal{D}^{\omega}$ is also left invariant by the operators in $\{\eta(X): X \in \mathfrak{g}\},{ }^{36}$ it follows from [59, Theorem 3.1] that the finite-dimensional real Lie algebra

$$
\left\{\left.\eta(X)\right|_{\mathcal{D}^{\omega}}: X \in \mathfrak{g}\right\} \subseteq \operatorname{End}\left(\mathcal{D}^{\omega}\right)
$$

is exponentiable. The inclusion $\overline{\left.\eta(X)\right|_{\mathcal{D}^{\omega}}} \subset \overline{\eta(X)}=\eta(X)$ together with dissipativity of $\eta(X)$ yields the equality $\overline{\left.\eta(X)\right|_{\mathcal{D}^{\omega}}}=\eta(X)=\overline{\left.\eta(X)\right|_{\mathcal{D}}}$, by [30, Theorem 3.1.15, $(3)$, page 177]. Therefore, $(\mathcal{D}, \mathfrak{g}, \eta)$ is also exponentiable. But then, again, since each generator $\eta(X)$ is a conservative operator having a dense set of analytic vectors contained in its domain, they actually generate groups of isometries.

Remark 2.11.1: Note that the conservativity hypothesis was just invoked in the last item of the proof. In order to obtain a more general exponentiation result (beyond the isometric case) one could, instead of imposing the conservativity hypothesis on the operators $\eta(X), X \in \mathfrak{g}$, replace it by the following (more general) condition: for each $X \in \mathfrak{g}$, there exist $\sigma_{x} \geq 0$ and $M_{x}>0$ such that, for all $n \in \mathbb{N},|\mu|>\sigma_{x}$ and $y \in \mathcal{D}$,

$$
\left\|(\mu I-\eta(X))^{n} y\right\| \geq M_{x}^{-1}\left(|\mu|-\sigma_{x}\right)^{n}\|y\| .
$$

Then, invoking [111, Theorem 1], [59, Theorem 3.1] and an adaptation of the proof of [30, Theorem 3.1.15, (3), page 177], ${ }^{37}$ just like it was done in the proof of the last item of Theorem 2.11, one obtains the following corollary:

Theorem 2.11.2 (Exponentiation - Banach Space Version, General Case): Let $\mathcal{X}$ be a Banach space, $\mathfrak{g}$ a real finite-dimensional Lie algebra with an ordered basis $\left(X_{k}\right)_{1 \leq k \leq d},(\mathcal{D}, \mathfrak{g}, \eta)$ a representation of $\mathfrak{g}$ by closed linear operators on $\mathcal{X}$, where $B_{k}:=\eta\left(X_{k}\right), 1 \leq k \leq d$, and $H_{m}$ an element of order $m \geq 2$ of $\mathfrak{U}\left(\eta_{\mathcal{D}}[\mathfrak{g}]\right)_{\mathbb{C}}$, where $\mathfrak{U}\left(\eta_{\mathcal{D}}[\mathfrak{g}]\right)_{\mathbb{C}}:=\mathfrak{U}\left(\eta_{\mathcal{D}}[\mathfrak{g}]\right)+i \mathfrak{U}\left(\eta_{\mathcal{D}}[\mathfrak{g}]\right)$ is the complexification of $\mathfrak{U}\left(\eta_{\mathcal{D}}[\mathfrak{g}]\right)$. Suppose that the following hypotheses are valid:

1. for each $X \in \mathfrak{g}$, there exist $\sigma_{x} \geq 0$ and $M_{x}>0$ such that

$$
\left\|(\mu I-\eta(X))^{n} y\right\| \geq M_{x}^{-1}\left(|\mu|-\sigma_{x}\right)^{n}\|y\|,
$$

for all $n \in \mathbb{N},|\mu|>\sigma_{x}$ and $y \in \mathcal{D}$;

\footnotetext{
${ }^{36}$ To see $C^{\omega}(\eta)$ is left invariant by the $B_{k}$ 's an analogous argument used to prove that the series obtained by term-by-term differentiation of a complex absolutely convergent power series is still absolutely convergent, and with the same radius of convergence, can be performed.

${ }^{37}$ Namely, the following theorem is also true: Let $S$ be a densely defined closable linear operator on the Banach space $\mathcal{Y}$. If $\bar{S}$ generates a strongly continuous semigroup $t \longmapsto S(t)$ satisfying

$$
\|S(t) y\| \leq M \exp (w t)\|y\|, \quad y \in \mathcal{Y}, t>0,
$$

for constants $M \geq 1$ and $w \geq 0$, and $T$ is an extension of $\bar{S}$ such that $\mu_{0} I-T$ is an injective operator, for some $\mu_{0}>w$, then $\bar{S}=T$.
} 
2. $-H_{m}$ is an infinitesimal pregenerator of a strongly continuous semigroup $t \longmapsto S(t)$ satisfying $S(t)[\mathcal{X}] \subseteq \mathcal{D}$, for each $t>0$ and, for each $n \in \mathbb{N}$ satisfying $0<n \leq m-1$, there exists $C_{n}>0$ for which the estimates

$$
\rho_{n}(S(t) y) \leq C_{n} t^{-\frac{n}{m}}\|y\|
$$

are verified for all $t \in(0,1]$ and all $y \in \mathcal{X}$, where $B_{0}:=I$ and

$$
\rho_{n}(x):=\max \left\{\left\|B_{i_{1}} \ldots B_{i_{k}} \ldots B_{i_{n}} x\right\|: 1 \leq k \leq n, 0 \leq i_{k} \leq d\right\},
$$

for all $x \in \mathcal{D}$.

Then, $(\mathcal{D}, \mathfrak{g}, \eta)$ exponentiates.

Using Corollary 2.10, it is possible to obtain a "locally convex space version" of Theorem 2.11 , by making just a few adjustments on its proof. Hence, the following generalization of Theorem 2.11 is true:

Theorem 2.12 (Exponentiation - Locally Convex Space Version, I): Let $\mathcal{X}$ be a complete Hausdorff locally convex space, $\mathfrak{g}$ a real finite-dimensional Lie algebra with an ordered basis $\mathcal{B}:=\left(X_{k}\right)_{1 \leq k \leq d},(\mathcal{D}, \mathfrak{g}, \eta)$ a representation of $\mathfrak{g}$ by closed linear operators on $\mathcal{X}$ and $H_{m}$ an element of order $m \geq 2$ of $\mathfrak{U}\left(\eta_{\mathcal{D}}[\mathfrak{g}]\right)_{\mathbb{C}}$. Define the subspace of projective analytic vectors for $\eta$ as

$$
C_{\leftarrow}^{\omega}(\eta):=\left\{x \in \mathcal{X}_{\infty}: \sum_{n=0}^{+\infty} \frac{\rho_{p, n}(x)}{n !} s_{x, p}^{n}<\infty, \text { for some } s_{x, p}>0, \text { for all } p \in \Gamma_{m}\right\},
$$

$\mathcal{D}^{\omega}:=\mathcal{D} \cap C_{\leftarrow}^{\omega}(\eta)$ and assume the following hypotheses:

1. each $B_{k}:=\eta\left(X_{k}\right)$ is a $\Gamma_{k}$-conservative operator, where $\Gamma_{k}$ is a fundamental system of seminorms for $\mathcal{X}$ - therefore, it is a closable operator, by Corollary 1.3.3;

2. $-H_{m}$ is an infinitesimal pregenerator of an equicontinuous semigroup $t \longmapsto S(t)$ satisfying $S(t)[\mathcal{X}] \subseteq \mathcal{D}$, for all $t \in(0,1]$ and, being a pregenerator of an equicontinuous semigroup, let $\Gamma_{m}$ be a fundamental system of seminorms for $\mathcal{X}$ with respect to which the operator $-H_{m}$ has the (KIP), is $\Gamma_{m}$-dissipative and $S$ is $\Gamma_{m}$-contractively equicontinuous;

3. define

$$
\Gamma_{\mathcal{B}}:=\bigcup_{1 \leq k \leq d} \Gamma_{k}
$$

suppose

$$
\Gamma_{\mathcal{B}} \subseteq \Gamma_{m}
$$


and that, for each $p \in \Gamma_{m}$ and $n \in \mathbb{N}$ satisfying $0<n \leq m-1$, there exists $C_{p, n}>0$ for which the estimates

$$
\rho_{p, n}(S(t) y) \leq C_{p, n} t^{-\frac{n}{m}} p(y)
$$

are verified for all $t \in(0,1]$ and $y \in \mathcal{X}$, where $B_{0}:=I$ and

$$
\rho_{p, n}(x):=\max \left\{p\left(B_{i_{1}} \ldots B_{i_{k}} \ldots B_{i_{n}} x\right): 1 \leq k \leq n, 0 \leq i_{k} \leq d\right\}, \quad x \in \mathcal{D} .
$$

Then: (a) The Lie algebra $\left\{\left.\eta(X)\right|_{D^{\omega}}: X \in \mathfrak{g}\right\}$ has a dense subspace of projective analytic vectors and is exponentiable to a strongly continuous locally equicontinuous representation of a Lie group. (b) If, instead, one has the stronger hypotheses that each $\eta(X)$ is $\Gamma_{X^{-}}$ conservative, where $\Gamma_{X}$ is a fundamental system of seminorms for $\mathcal{X}$, and

$$
\Gamma:=\bigcup_{X \in \mathfrak{g}} \Gamma_{X} \subseteq \Gamma_{m}
$$

then $(\mathcal{D}, \mathfrak{g}, \eta)$ exponentiates to a representation by equicontinuous one-parameter groups, and the Lie algebra $\eta_{\mathcal{D}}[\mathfrak{g}]$ has a dense set of projective analytic vectors.

(c) If, instead, it happens that all of the operators $\eta\left(X_{k}\right), 1 \leq k \leq d$, are $\Gamma_{m^{-}}$ conservative, then $(\mathcal{D}, \mathfrak{g}, \eta)$ exponentiates to a representation by $\Gamma_{m}$-isometrically equicontinuous one-parameter groups.

Proof of Theorem 2.12: Since $-H_{m}$ is a pregenerator of an equicontinuous group, the resolvent $\mathrm{R}\left(1,-\delta \overline{H_{m}}\right)$ is well-defined for all $0<\delta<1$ and the identity

$$
\mathrm{R}\left(1,-\delta \overline{H_{m}}\right)=\int_{0}^{+\infty} \exp (-t) S_{\delta t}(y) d t, \quad y \in \mathcal{X}
$$

holds - see [126, Theorem 1, page 240] or [8, Theorem 3.3, page 172] - with

$$
\int_{0}^{+\infty} \exp (-t) p\left(S_{\delta t}(y)\right) d t<\infty
$$

for all $p \in \Gamma_{m}$. Then, by the estimates of the hypotheses, one finds analogously that

$$
\begin{gathered}
\int_{0}^{+\infty} \exp (-t) p\left(B^{\mathrm{u}} S_{\delta t}(y)\right) d t \\
\leq C_{p, n}\left(\sqrt[m]{(m-n) !} \frac{m}{m-n}+I_{0} \frac{m}{m-n}+2 \sqrt[m]{n !}\right) \delta^{-\frac{n}{m}} p(y),
\end{gathered}
$$


for all $y \in \mathcal{X}$. As before, closedness of each operator $B_{k}$ shows that $\left(1+\delta \overline{H_{m}}\right)^{-1}(y) \in$ Dom $B^{\mathrm{u}}$ and

$$
B^{\mathrm{u}}\left(1+\delta \overline{H_{m}}\right)^{-1}(y)=\int_{0}^{+\infty} \exp (-t) B^{\mathrm{u}} S_{\delta t}(y) d t
$$

Hence, the estimates obtained show that the inequality

$$
\rho_{p, n}(y) \leq \epsilon^{m-n} p\left(H_{m}(y)\right)+\frac{E_{p, n}}{\epsilon^{n}} p(y)
$$

is valid for every $p \in \Gamma_{m}, 0<\epsilon \leq 1,0<n \leq m-1$ and $y \in \mathcal{D}$, for some $E_{p, n}>0$. A very important observation is that (2.12.2) proves, in particular, that the operators $\left(B_{k}\right)_{1 \leq k \leq d}$ possess the (KIP) with respect to $\Gamma_{m}$, so the extra hypothesis $\Gamma_{\mathcal{B}} \subseteq \Gamma_{m}$ guarantees that these basis elements possess the (KIP) relatively to $\Gamma_{\mathcal{B}}$, a property which must be verified if one wants to apply Corollary 2.10 .

The rest of the adaptation of Theorem 2.11 goes as follows:

- Given $p \in \Gamma_{m}$ and $q \in \mathbb{N}, q \geq 1$, there exists a strictly positive constant $K_{p, q}$ such that

$$
\rho_{p, n}(S(t) y) \leq K_{p, q} \sup _{0 \leq j \leq q} p\left(H_{m}^{j}(y)\right),
$$

for all $y \in \mathcal{D},(q-1)(m-1)<n \leq q(m-1)$ and $t \in(0,1]$. This implies, in particular, that given $q \in \mathbb{N}, q \geq 1$, and $y \in \mathcal{D}$, there exists $K_{y, p, q}>0$ satisfying $\rho_{p, n}(S(t) y) \leq K_{y, p, q}$, for all $t \in(0,1]$ and $(q-1)(m-1)<n \leq q(m-1)$ :

The proof is done by induction on $q$, using (2.12.2), in perfect analogy with what is done in Theorem 2.11 .

- For each $p \in \Gamma_{m}$ there exist $K_{p}, L_{p} \geq 1$ such that, given $q \in \mathbb{N}, q \geq 1$ and $(q-$ 1) $(m-1)<n \leq q(m-1)$, there exists a strictly positive constant $C_{p, n}$ defined by $C_{p, n}:=K_{p} L_{p}^{n} n$ ! satisfying

$$
\rho_{p, n}(S(t) y) \leq C_{p, n} t^{-\frac{n}{m}} p(y), \quad y \in \mathcal{X}, t \in(0,1] .
$$

More precisely, there exist $K_{p}, L_{p} \geq 1$ such that

$$
\rho_{p, n}(S(t) y) \leq K_{p} L_{p}^{n} n ! t^{-\frac{n}{m}} p(y),
$$

for all $n \in \mathbb{N}, n \geq 1, y \in \mathcal{X}$ and $t \in(0,1]$ :

The idea is, again, to proceed by induction on $q$, and the proof goes practically unchanged, evaluating seminorms on the vectors, instead of norms. 
- It is now that the context of locally convex spaces really makes a difference, and where Corollary 2.10 will be needed. As before, define $\mathcal{X}_{0}:=\bigcup_{0<t \leq 1} S(t)[\mathcal{X}]$. Since $S$ is strongly continuous, $\mathcal{X}_{0}$ is dense in $\mathcal{X}$. Using the estimates from the previous item shows that

$$
\mathcal{X}_{0} \subseteq C_{\leftarrow}^{\omega}(\eta) \cap \mathcal{D}=\mathcal{D}^{\omega}:
$$

fix $p \in \Gamma_{m}, 0<t \leq 1$ and $y \in \mathcal{X}$; since

$$
\sum_{n=0}^{+\infty} \frac{\rho_{p, n}(S(t) y)}{n !} r^{n} \leq K_{p}\|y\| \sum_{n=0}^{+\infty} L_{p}^{n} t^{-\frac{n}{m}} r^{n}, \quad r>0,
$$

if one chooses $r$ so that

$$
r<\frac{1}{L_{p} t^{-\frac{1}{m}}}
$$

then the latter series converges. This proves the inclusion and, therefore, $\mathcal{D}^{\omega}$ is dense in $\mathcal{X}$. Each $\left.B_{k}\right|_{\mathcal{D}^{\omega}}$ is a $\Gamma_{k^{-}}$-conservative operator having the (KIP) with respect to $\Gamma_{k}$, so by Lemma 1.4.3 they are all infinitesimal pregenerators of equicontinuous groups. Just as in the Banach space setting the inclusion $C_{\leftarrow}^{\omega}(\eta) \subseteq C_{\leftarrow}^{\omega}(\eta(X))$ is valid, for every $X \in \mathfrak{g}$. Also, $\mathcal{D}^{\omega}$ is left invariant by the operators in $\{\eta(X): X \in \mathfrak{g}\}$. Therefore, since all the elements $\left\{\left.B_{k}\right|_{\mathcal{D}^{\omega}}\right\}_{1 \leq k \leq d}$ possess the (KIP) with respect to $\Gamma_{\mathcal{B}}$, Corollary 2.10 becomes applicable, so the finite-dimensional real Lie algebra $\left\{\left.\eta(X)\right|_{\mathcal{D}^{\omega}}: X \in \mathfrak{g}\right\}$ exponentiates to a strongly continuous locally equicontinuous representation of a Lie group.

Now, suppose that every $\eta(X), X \in \mathfrak{g}$, is a $\Gamma_{X}$-conservative operator and that the inclusion

$$
\Gamma:=\bigcup_{X \in \mathfrak{g}} \Gamma_{X} \subseteq \Gamma_{m}
$$

holds. Each operator $\overline{\left.\eta(X)\right|_{\mathcal{D}} \text { is }}$ is already known to generate a strongly continuous locally equicontinuous group, but the stronger hypotheses just assumed also imply that $\overline{\left.\eta(X)\right|_{\mathcal{D}^{\omega}}}$ is a $\Gamma_{X}$-conservative operator having the (KIP) with respect to $\Gamma_{X}$, for all $X \in \mathfrak{g}$. Hence, since all of them have a dense set of projective analytic vectors (by the argument in the preceding paragraph), the stronger conclusion that each $\overline{\left.\eta(X)\right|_{\mathcal{D}^{\omega}}}$ is the generator of a $\Gamma_{X}$-isometrically equicontinuous group follows, by Lemma 1.4.3. The inclusion $\overline{\left.\eta(X)\right|_{\mathcal{D}^{\omega}}} \subset \overline{\eta(X)}=\eta(X)$ holds, for every $X \in \mathfrak{g}$, so all that remains to be shown is that the generator of an equicontinuous semigroup has no proper dissipative extensions, an objective which may be accomplished by an adaptation of the argument in [30, Theorem 3.1.15, (3), page 177]: let $T_{X}$ be a proper extension of $\overline{\left.\eta(X)\right|_{\mathcal{D}^{\omega}}}$, and let $x \in \operatorname{Dom} T_{X} \backslash \operatorname{Dom} \overline{\left.\eta(X)\right|_{\mathcal{D}^{\omega}}}$. Since $\overline{\left.\eta(X)\right|_{\mathcal{D}^{\omega}}}$ is a $\Gamma_{X}$-dissipative operator that generates an equicontinuous semigroup (actually, it is a $\Gamma_{X}$-conservative operator that generates an equicontinuous group, but this additional 
information will not be needed), by [3, Proposition 3.13] and [3, Theorem 3.14] (see Section 1.4) the equality

$$
\mathcal{X}=\overline{\operatorname{Ran}\left(I-\left.\eta(X)\right|_{\mathcal{D}^{\omega}}\right)}=\operatorname{Ran}\left(I-\overline{\left.\eta(X)\right|_{\mathcal{D}^{\omega}}}\right)
$$

must hold. Therefore, there must be $y \in \operatorname{Dom}\left(I-\overline{\eta(X) \mid \mathcal{D}^{\omega}}\right)$ such that

$$
\left(I-T_{X}\right) x=\left(I-\overline{\left.\eta(X)\right|_{\mathcal{D}^{\omega}}}\right) y=\left(I-T_{X}\right) y .
$$

But, then $\left(I-T_{X}\right)(x-y)=0$, so $\Gamma_{X}$-dissipativity of $T_{X}$ together with the fact that $\mathcal{X}$ is Hausdorff implies $x=y \in \operatorname{Dom} \overline{\left.\eta(X)\right|_{\mathcal{D}^{\omega}}}$, contradicting the hypothesis on $x$. This proves that $\overline{\left.\eta(X)\right|_{\mathcal{D}^{\omega}}}$ does not have any proper $\Gamma_{X}$-dissipative extensions and that the equality $\overline{\left.\eta(X)\right|_{\mathcal{D}^{\omega}}}=\eta(X)=\overline{\left.\eta(X)\right|_{\mathcal{D}}}$ holds, for every $X \in \mathfrak{g}$, so $(\mathcal{D}, \mathfrak{g}, \eta)$ is exponentiable to a representation by equicontinuous one-parameter groups (and, for each $X \in \mathfrak{g}, \eta(X)$ is the generator of a $\Gamma_{X}$-isometrically equicontinuous group).

Now, suppose, instead of hypothesis 1., that the stronger assumption that all of the operators $\eta\left(X_{k}\right), 1 \leq k \leq d$, are $\Gamma_{m}$-conservative, holds. Using the argumentation of the last paragraph yields

$$
\overline{B_{k} \mid \mathcal{D}^{\omega}}=B_{k}, \quad 1 \leq k \leq d,
$$

so that each $B_{k}$ is the generator of a $\Gamma_{m}$-isometrically equicontinuous group, and a repetition of the argument in Corollary 2.8 proves that all of the operators $\eta(X)$, $X \in \mathfrak{g}$, are generators of $\Gamma_{m}$-isometrically equicontinuous one-parameter groups.

The next objective of this section is to give necessary conditions for the exponentiability problem treated so far.

\section{- Strongly Elliptic Operators - Necessary Conditions for Exponentiation}

Theorem 2.13 (Strongly Elliptic Operators on Locally Convex Spaces): Let $(\mathcal{X}, \Gamma)$ be a complete Hausdorff locally convex space and $\left(\mathcal{X}_{\infty}, \mathfrak{g}, \eta\right)$ an exponentiable representation by closed operators, where $\mathcal{B}:=\left(X_{k}\right)_{1 \leq k \leq d}$ is an ordered basis of the finitedimensional real Lie algebra $\mathfrak{g}, \eta\left(X_{k}\right):=B_{k}$ and

$$
\mathcal{X}_{\infty}:=\bigcap_{n=1}^{+\infty} \bigcap\left\{\operatorname{Dom}\left[B_{i_{1}} \ldots B_{i_{k}} \ldots B_{i_{n}}\right]: 1 \leq k \leq n, 1 \leq i_{k} \leq d\right\} .
$$

Let $G$ be the underlying simply connected Lie group such that $V: G \longrightarrow \mathcal{L}(\mathcal{X})$ is the strongly continuous locally equicontinuous representation satisfying the property that $d V(X)=$ $\overline{\left.\eta(X)\right|_{\mathcal{X}_{\infty}}}=\eta(X)$, for all $X \in \mathfrak{g}$ - in particular, $d V\left(X_{k}\right)=\eta\left(X_{k}\right)=B_{k}$, for all $1 \leq k \leq d$, and $\mathcal{X}_{\infty}=C^{\infty}(V)$ - see Subsection 1.2.2. Suppose that: 
1. the operators $\left(B_{k}\right)_{1 \leq k \leq d}$ are all $\Gamma$-conservative;

2. each $d V\left(X_{k}\right), 1 \leq k \leq d$, is the infinitesimal generator of an equicontinuous group $V_{k}(t):=V\left(\exp t X_{k}\right)$.

Define $H_{m} \in \mathfrak{U}(\partial V[\mathfrak{g}])_{\mathbb{C}}$ by

$$
H_{m}:=\sum_{\alpha ;|\alpha| \leq m} c_{\alpha} \partial V\left(X_{1}\right)^{\alpha_{1}} \ldots \partial V\left(X_{k}\right)^{\alpha_{k}} \ldots \partial V\left(X_{d}\right)^{\alpha_{d}}
$$

so Dom $H_{m}=\mathcal{X}_{\infty}$. Suppose $H_{m}$ is a strongly elliptic operator on $\mathcal{X}$ and that $-H_{m}$ is a $\Gamma$-dissipative operator. Then, $-H_{m}$ is an infinitesimal pregenerator of a $\Gamma$-contractively equicontinuous semigroup $S:[0,+\infty) \longrightarrow \mathcal{L}(\mathcal{X})$ such that $S(t)[\mathcal{X}] \subseteq \mathcal{X}_{\infty}$, for all $t>0$ and, for each $p \in \Gamma$ and $n \in \mathbb{N}$ satisfying $0<n \leq m-1$, there exists $C_{p, n}>0$ validating the estimates

$$
\rho_{p, n}(S(t) y) \leq C_{p, n} t^{-\frac{n}{m}} p(y),
$$

for every $t \in(0,1]$ and $y \in \mathcal{X}$. Moreover, if $H_{m}=-\sum_{k=1}^{d} \partial V\left(X_{k}\right)^{2}$, then the hypothesis of $\Gamma$-dissipativity is superfluous.

Proof of Theorem 2.13: The first task of the proof is to obtain the global (KIP) for $V$ with respect to $\Gamma$ or, in other words, to obtain the inclusions

$$
V[G]\left[N_{p}\right] \subseteq N_{p}, \quad p \in \Gamma
$$

To this end, it is sufficient to repeat the argument inside of the proof of Corollary 2.8 to obtain $p(V(g) x)=p(x)$, for all $p \in \Gamma, g \in G$ and $x \in \mathcal{X}$, so that the global (KIP) $V[G]\left[N_{p}\right] \subseteq N_{p}$ holds, for all $p \in \Gamma$. This shows that the induced strongly continuous locally equicontinuous group representation $V_{p}: G \longrightarrow \mathcal{X}_{p}$, defined at each fixed $g \in G$ as the extension by density of the bounded isometric linear operator $V_{p}(g):[y]_{p} \longmapsto[V(g) y]_{p}$, is well-defined, for all $p \in \Gamma$, and that it is a representation by isometries. Because of the arguments just exposed, it follows that if $H_{2}:=-\sum_{k=1}^{d} \partial V\left(X_{k}\right)^{2}$, then the dissipativity hypothesis on $-H_{2}=: \Delta$ is superfluous: fix $p \in \Gamma$. By what was just proved in this paragraph, the operators $\partial V(X), X \in \mathfrak{g}$, have the (KIP) with respect to $\Gamma$. Note first that

$$
\begin{gathered}
\left\|\left(I-\lambda^{2}\left(\partial V\left(X_{k}\right)\right)_{p}^{2}\right)[x]_{p}\right\|_{p}=\left\|\left(I-\lambda\left(\partial V\left(X_{k}\right)\right)_{p}\right)\left(I+\lambda\left(\partial V\left(X_{k}\right)\right)_{p}\right)[x]_{p}\right\|_{p} \\
\geq\left\|[x]_{p}\right\|_{p}, \quad 1 \leq k \leq d, \lambda \in \mathbb{R}, x \in \mathcal{X}_{\infty}
\end{gathered}
$$

by conservativity of the operators $\left(\partial V\left(X_{k}\right)\right)_{p}$. This proves $\left(\partial V\left(X_{k}\right)\right)_{p}^{2}$ is a dissipative operator, for all $1 \leq k \leq d$. Fix $[x]_{p} \in \operatorname{Dom} \Delta_{p}$. By a characterization of dissipativity on Banach spaces (see pages 174 and 175 of [30]), if $f \in\left(\mathcal{X}_{p}\right)^{\prime}$ is a tangent functional at $[x]_{p} \in$ 
Dom $\Delta_{p}$ (in other words, a bounded linear functional such that $\left|f\left([x]_{p}\right)\right|=\|f\|\left\|[x]_{p}\right\|_{p}$, where $\|f\|$ denotes the operator norm of $f$ ), then

$$
\operatorname{Re} f\left(\left(\partial V\left(X_{k}\right)\right)_{p}^{2}\left([x]_{p}\right)\right) \leq 0, \quad 1 \leq k \leq d .
$$

Therefore, by linearity, $\operatorname{Re} f\left(\Delta_{p}\left([x]_{p}\right)\right) \leq 0$, proving that $\Delta_{p}$ is a dissipative operator. But $p \in \Gamma$ is arbitrary, so this shows $\Delta$ is $\Gamma$-dissipative.

Since all of the operators $\partial V\left(X_{k}\right), 1 \leq k \leq d$, possess the (KIP) with respect to $\Gamma$, it follows that $-H_{m}$ also has this property. Fix $p \in \Gamma$ and $1 \leq k \leq d$. The inclusion $\left(\partial V\left(X_{k}\right)\right)_{p} \subset \partial V_{p}\left(X_{k}\right)$ holds, because $\pi_{p}\left[C^{\infty}(V)\right] \subseteq C^{\infty}\left(V_{p}\right)$ and, if $y \in C^{\infty}(V)$, then

$$
\left\|\frac{V_{p}\left(\exp t X_{k}\right)\left([y]_{p}\right)-[y]_{p}}{t}-\left(\partial V\left(X_{k}\right)\right)_{p}\left([y]_{p}\right)\right\|_{p}=p\left(\frac{V\left(\exp t X_{k}\right) y-y}{t}-\partial V\left(X_{k}\right)(y)\right)
$$

goes to 0 , as $t \longrightarrow 0$. Hence, defining

$$
H_{p}:=\sum_{\alpha ;|\alpha| \leq m} c_{\alpha} \partial V_{p}\left(X_{1}\right)^{\alpha_{1}} \ldots \partial V_{p}\left(X_{k}\right)^{\alpha_{k}} \ldots \partial V_{p}\left(X_{d}\right)^{\alpha_{d}},
$$

the inclusion

$$
\left(-H_{m}\right)_{p}=-\sum_{\alpha ;|\alpha| \leq m} c_{\alpha}\left(\partial V\left(X_{1}\right)\right)_{p}^{\alpha_{1}} \ldots\left(\partial V\left(X_{k}\right)\right)_{p}^{\alpha_{k}} \ldots\left(\partial V\left(X_{d}\right)\right)_{p}^{\alpha_{d}} \subset-H_{p}
$$

also holds. Now, [109, Theorem 5.1, page 30] says that $-H_{p}$ is a pregenerator of a strongly continuous semigroup $S_{p}$ on $\mathcal{X}_{p}$. Hence, by [8, Theorem 2.5] (see Section 1.4), the projective limit of the family $\left\{\overline{H_{p}}\right\}_{p \in \Gamma}$, which will be denoted by $\tilde{H}_{m}$, is such that $-\tilde{H}_{m}$ is the generator of a $\Gamma$-semigroup $S:[0,+\infty) \longrightarrow \mathcal{L}_{\Gamma}(\mathcal{X})$ on $\lim _{\longleftarrow} \mathcal{X}_{p}$, which is the projective limit of the semigroups $\left\{S_{p}\right\}_{p \in \Gamma}$. Since each $-\overline{H_{p}}$ is dissipative, by hypothesis, it follows by the Feller-Miyadera-Phillips Theorem together with [3, Proposition 3.11 (iii)] (applied to Banach spaces) that $-\overline{H_{p}}$ is actually the generator of a contraction semigroup. Therefore, $-\tilde{H}_{m}$ must be the generator of a $\Gamma$-contractively equicontinuous semigroup on $\lim _{\mathcal{X}_{p}}$. It should be noted, at this point, that all of the results proved on $\lim _{\mathcal{X}}$ carry over to $\mathcal{X}$ (and vice-versa) since, by Lemma 1.7.1, $\mathcal{X}$ and $\varliminf_{\text {im }} \mathcal{X}_{p}$ are isomorphic locally convex spaces. Therefore, in what follows, $\mathcal{X}$ and $\lim _{\longleftarrow} \mathcal{X}_{p}$ will be treated as if they were the same space. Hence, the relation

$$
-H_{m}=-\lim _{\longleftarrow}\left(H_{m}\right)_{p} \subset-\lim _{\longleftarrow} \overline{H_{p}}=-\tilde{H}_{m}
$$

holds.

To conclude that $-\overline{H_{m}}$ is the generator of a $\Gamma$-contractively equicontinuous semigroup, it suffices to show that $-\overline{H_{m}}=-\tilde{H}_{m}$, an equality which will be obtained after the next 
paragraph.

To prove the inclusion $S(t)[\mathcal{X}] \subseteq \mathcal{X}_{\infty}$, for all $t>0$, note first that, if $1 \leq k \leq d$ and $y \in \mathcal{X}$, then the function $t \longmapsto V\left(\exp t X_{k}\right) y$ is infinitely differentiable on $[0,+\infty)$ if, and only if, $t \longmapsto V_{p}\left(\exp t X_{k}\right)\left([y]_{p}\right)$ is infinitely differentiable on $[0,+\infty)$, for all $p \in \Gamma$. Hence, using the alternative description of smooth vectors for a strongly continuous locally equicontinuous representation proved in 1.2.2, one concludes that $y$ belongs to $C^{\infty}(V)$ if, and only if, $[y]_{p}$ belongs to $C^{\infty}\left(V_{p}\right)$, for every $p \in \Gamma$. But [109, Theorem 5.1] says, in particular, that $S_{p}(t)\left[\mathcal{X}_{p}\right] \subseteq C^{\infty}\left(V_{p}\right)$, for all $t>0$. Therefore, $[S(t) y]_{p}=S_{p}\left([y]_{p}\right)$ belongs to $C^{\infty}\left(V_{p}\right)$, for all $p \in \Gamma$ and $t>0$, so $S(t) y$ must belong to $\mathcal{X}_{\infty}$, for each fixed $t>0$.

The argument in the previous paragraph shows, in particular, that $S(t)\left[\mathcal{X}_{\infty}\right] \subseteq \mathcal{X}_{\infty}$, for all $t>0$, so Lemma 1.2.4.3 says that $\mathcal{X}_{\infty}$ is a core for $-\tilde{H}_{m}$. Hence,

$$
-\tilde{H}_{m}=-\overline{\tilde{H}_{m} \mid \mathcal{X}_{\infty}}=-\overline{H_{m}}
$$

as claimed.

Finally, validity of 2.13.1 is a consequence of [109, Corollary 5.6, page 44], for if $p \in \Gamma$, $B^{\mathrm{u}}$ is a monomial of size $0<|\mathrm{u}|:=n \leq m-1$ in the generators $\left\{d V\left(X_{k}\right)\right\}_{1 \leq k \leq d}$ and $\left(B^{\mathrm{u}}\right)_{p}$ is the induced monomial in the quotient $\mathcal{X} / N_{p}$, then

$$
\begin{gathered}
p\left(B^{\mathrm{u}} S(t) y\right)=\left\|\left(B^{\mathrm{u}}\right)_{p} S_{p}(t)[y]_{p}\right\|_{p} \leq C_{p, n} t^{-\frac{n}{m}}\left\|[y]_{p}\right\|_{p} \\
=C_{p, n} t^{-\frac{n}{m}} p(y), \quad t \in(0,1], y \in \mathcal{X},
\end{gathered}
$$

for some constants $C_{p, n}>0$, since $(d V(X))_{p} \subset d V_{p}(X)$, for every $p \in \Gamma$ and $X \in \mathfrak{g}$.

Observation 2.13.1: In the same context of Theorem 2.13, it is possible to show that if the strongly continuous locally equicontinuous representation $V: G \longrightarrow \mathcal{L}(\mathcal{X})$ satisfies the condition that, for each $p \in \Gamma$, there exists $M_{p}>0$ and another $q \in \Gamma$ satisfying

$$
\sup _{g \in G} p(V(g) x) \leq M_{p} q(x), \quad x \in \mathcal{X}
$$

- this is a condition which is automatically satisfied if $G$ is compact - then hypothesis 1 of the theorem which asks for conservativity of the operators $\left(B_{k}\right)_{1 \leq k \leq d}$ with respect to the same $\Gamma$ is automatically fulfilled. To see this, fix $p \in \Gamma$. In a similar fashion of that done in Section 1.3, one may define the seminorm

$$
x \longmapsto \tilde{p}(x):=\sup _{g \in G} p(V(g) x)<\infty
$$

and see that

$$
p(x) \leq \tilde{p}(x) \leq M_{p} q(x) \leq M_{p} \tilde{q}(x), \quad x \in \mathcal{X}
$$


proving that the families $\Gamma$ and $\tilde{\Gamma}:=\{\tilde{p}: p \in \Gamma\}$ are equivalent. But even more, $\tilde{\Gamma}$ has the very useful property that

$$
\tilde{p}(V(h) x)=\sup _{g \in G} p(V(g h) x)=\sup _{g \in G} p(V(g) x)=\tilde{p}(x),
$$

for all $h \in G$ and $x \in \mathcal{X}$, so

$$
\tilde{p}(V(h) x)=\tilde{p}(x), \quad h \in G, x \in \mathcal{X} .
$$

Repeating the argument in Section 1.3 yields conservativity of the operators $\left\{d V\left(X_{k}\right)\right\}_{1 \leq k \leq d}$ with respect to $\tilde{\Gamma}$. Hence, the assertion follows.

\section{- Exponentiation in Locally Convex Spaces - Characterization}

Finally, it is possible to combine Theorems 2.12 and 2.13 to obtain a characterization of Lie algebras of linear operators which exponentiate to representations by $\Gamma$-isometrically equicontinuous one-parameter groups, generalizing [25, Theorem 3.9]:

Theorem 2.14 (Exponentiation - Locally Convex Space Version, II): $\operatorname{Let}(\mathcal{X}, \Gamma)$ be a complete Hausdorff locally convex space and $\left(\mathcal{X}_{\infty}, \mathfrak{g}, \eta\right)$ a representation by closed operators, where $\mathcal{B}:=\left(X_{k}\right)_{1 \leq k \leq d}$ is an ordered basis of the finite-dimensional real Lie algebra $\mathfrak{g}, \eta\left(X_{k}\right):=B_{k}$ and

$$
\mathcal{X}_{\infty}:=\bigcap_{n=1}^{+\infty} \bigcap\left\{\operatorname{Dom}\left[B_{i_{1}} \ldots B_{i_{k}} \ldots B_{i_{n}}\right]: 1 \leq k \leq n, 1 \leq i_{k} \leq d\right\} .
$$

Suppose that the operators $\left(B_{k}\right)_{1 \leq k \leq d}$ are $\Gamma$-conservative,

$$
H_{m}:=\sum_{\alpha ;|\alpha| \leq m} c_{\alpha} \eta_{\mathcal{X}_{\infty}}\left(X_{1}\right)^{\alpha_{1}} \ldots \eta_{\mathcal{X}_{\infty}}\left(X_{k}\right)^{\alpha_{k}} \ldots \eta_{\mathcal{X}_{\infty}}\left(X_{d}\right)^{\alpha_{d}}
$$

is an element of $\mathfrak{U}\left(\eta_{\mathcal{X}_{\infty}}[\mathfrak{g}]\right)_{\mathbb{C}}$ of order $m \geq 2$ which is strongly elliptic and that $-H_{m}$ is $\Gamma$-dissipative. ${ }^{38}$ Then, the following are equivalent:

1. $\left(\mathcal{X}_{\infty}, \mathfrak{g}, \eta\right)$ exponentiates to a strongly continuous locally equicontinuous representation $V: G \longrightarrow \mathcal{L}(\mathcal{X})$ of a simply connected Lie group $G$, having $\mathfrak{g}$ as its Lie algebra, such that $d V(X)=\overline{\left.\eta(X)\right|_{\mathcal{X}_{\infty}}}$ is the generator of a $\Gamma$-isometrically equicontinuous oneparameter group, for all $X \in \mathfrak{g}$;

\footnotetext{
${ }^{38}$ If $H_{m}=-\sum_{k=1}^{d} \eta_{\mathcal{X}_{\infty}}\left(X_{k}\right)^{2}$, then the dissipativity hypothesis on $-H_{m}$ will be superfluous, by the usual arguments.
} 
2. $-H_{m}$ is an infinitesimal pregenerator of a $\Gamma$-contractively equicontinuous semigroup $S:[0,+\infty) \longrightarrow \mathcal{L}(\mathcal{X})$ such that $S(t)[\mathcal{X}] \subseteq \mathcal{X}_{\infty}$, for all $t>0$ and, for each $p \in \Gamma$ and $n \in \mathbb{N}$ satisfying $0<n \leq m-1$, there exists $C_{p, n}>0$ for which

$$
\text { (2.14.1) } \quad \rho_{p, n}(S(t) y) \leq C_{p, n} t^{-\frac{n}{m}} p(y),
$$

where $t \in(0,1]$ and $y \in \mathcal{X}$.

Also, if one of the above conditions is fulfilled, then $\eta$ has a dense set of projective analytic vectors. 

Chapter 3

\section{Some Applications to Locally Convex Algebras}

\section{Definitions, Examples and a Few Structure Theorems}

Definition 3.1: Let $\mathcal{A}$ be a Hausdorff locally convex space (over $\mathbb{C}$ ) equipped with an associative product which is compatible with the sum via the distributive rules $(a+b) c=a c+b c$, $a(b+c)=a b+a c$, for all $a, b, c \in \mathcal{A}$. Also, suppose that the multiplication is separately continuous: for each fixed $a \in \mathcal{A}$, the maps $b \longmapsto a b$ and $b \longmapsto b a$ are continuous. Then, $\mathcal{A}$ is called a locally convex algebra. If the underlying vector space of $\mathcal{A}$ is a complete (respectively, sequentially complete) Hausdorff locally convex space, then it said to be a complete (respectively, sequentially complete) locally convex algebra. It may also happen that the product satisfies a stronger continuity assumption: if the map $(a, b) \longmapsto a b$ from $\mathcal{A} \times \mathcal{A}$ to $\mathcal{A}$ is continuous, then the product is said to be jointly continuous [66, page 24]. If $p$ is a seminorm on $\mathcal{A}$ satisfying $p(a b) \leq p(a) p(b)$, for all $a, b \in \mathcal{A}$, then it is said to be submultiplicative, or an $\boldsymbol{m}$-seminorm. Therefore, if $\mathcal{A}$ possesses a fundamental system of submultiplicative seminorms, then its product is jointly continuous. A locally convex algebra having a fundamental system of m-seminorms is called an m-convex algebra. ${ }^{1}$ If an m-convex algebra is complete, then it is called an Arens-Michael algebra. If, moreover, the topology of an Arens-Michael algebra is defined by a countable family of seminorms, then it is called a Fréchet algebra.

Suppose $\mathcal{A}$ is a locally convex algebra equipped with an involution $*$, which is, by definition, an antilinear map on $\mathcal{A}$ satisfying $(a b)^{*}=b^{*} a^{*}$ and $\left(a^{*}\right)^{*}=a$, for all $a, b \in \mathcal{A}$. Then, $\mathcal{A}$ is called a locally convex algebra with involution or an involutive locally

\footnotetext{
${ }^{1}$ As it was just noted, above, every m-convex algebra has a jointly continuous product. The converse, however, does not hold: [6, Theorem 2] shows a concrete example of a commutative, metrizable and complete locally convex algebra with jointly continuous multiplication which is not an m-convex algebra (this algebra is known as Arens algebra). See also [90, page 10] and [90, Proposition 4.3, page 18].
} 
convex algebra. If $*: a \longmapsto a^{*}$ is a continuous map, $\mathcal{A}$ is called a locally convex *-algebra. A seminorm $p$ on an involutive algebra $\mathcal{A}$ is said to be a*-seminorm if $p\left(a^{*}\right)=p(a)$, for all $a \in \mathcal{A}$. By the consequence of a theorem ([53, Theorem 3.7, page 32]), there always exists a fundamental system of *-seminorms which generates the topology of a locally convex $*$-algebra. If a *-seminorm $p$ is also submultiplicative, then it is called an $m^{*}$-seminorm. A locally convex *-algebra whose topology is generated by a family of $m^{*}$ seminorms is called an $\boldsymbol{m}^{*}$-convex algebra and, if such *-algebra is, moreover, complete, it is called an Arens-Michael *-algebra. If the topology of an Arens-Michael *-algebra can be generated by a countable family of seminorms, then it is called a Fréchet $*$-algebra.

Some interesting examples of locally convex $*$-algebras will now be given:

1. Arens-Michael *-algebras: The function spaces $C^{\infty}\left(\mathbb{R}^{n}\right), \mathcal{O}(\mathbb{C}), \mathcal{D}\left(\mathbb{R}^{n}\right), \mathcal{S}\left(\mathbb{R}^{n}\right)$ and $C\left(\mathbb{R}^{n}\right)$, already introduced in Chapter 1 , are all examples of commutative ArensMichael $*$-algebras. Let $\mathcal{A}$ be a unital noncommutative locally $\mathrm{C}^{*}$-algebra (see the item just below) and $\mathcal{M}$ a smooth manifold. Then, the space of smooth $\mathcal{A}$-valued functions

$$
C^{\infty}(M, \mathcal{A}):=\left\{f: \mathcal{M} \longrightarrow \mathcal{A}: x^{\prime} \circ f \in C^{\infty}(\mathcal{M}), x^{\prime} \in \mathcal{A}^{\prime}\right\}
$$

is a noncommutative Arens-Michael $*$-algebra (which is not a locally $\mathrm{C}^{*}$-algebra) [53, 21.17(4), page 278].

2. The locally $\mathbf{C}^{*}$-algebras: A complete locally convex *-algebra $\mathcal{A}$ whose topology $\tau$ is generated by a directed family $\Gamma$ of seminorms satisfying the properties:

$$
\begin{aligned}
& \text { (i) } p(a b) \leq p(a) p(b), \quad \text { (ii) } p\left(a^{*}\right)=p(a), \\
& \text { (iii) } p\left(a^{*} a\right)=p(a)^{2}, \quad p \in \Gamma, a, b \in \mathcal{A},
\end{aligned}
$$

is called a locally $\mathbf{C}^{*}$-algebra - a seminorm $p$ satisfying (iii) is called a $\mathrm{C}^{*}$-seminorm. ${ }^{2}$ It is said to be unital if it possesses an identity: in other words, if it has an element 1 satisfying the property $1 \cdot a=a \cdot 1=a$, for all $a \in \mathcal{A}$. Hence, the concept of a locally $\mathrm{C}^{*}$-algebra generalizes that of a $\mathrm{C}^{*}$-algebra. The terminology used to refer to locally $\mathrm{C}^{*}$-algebras varies in the literature, and they are also called pro- $\mathbf{C}^{*}$-algebras or LMC*-algebras (see [53, page 99] and [100], which calls them pro-C*-algebras; the terminology "locally $\mathrm{C}^{*}$-algebra" was apparently introduced by [70]; [13] calls them $\mathrm{LMC}^{*}$-algebras). If $\mathcal{A}$ is a locally $\mathrm{C}^{*}$-algebra, the algebra of the so-called "bounded elements", defined as

$$
b(\mathcal{A}):=\left\{a \in \mathcal{A}: \sup _{p \in \Gamma} p(a)<\infty\right\},
$$

\footnotetext{
${ }^{2}$ As a consequence of [53, Theorem 7.2 , page 101], a $\mathrm{C}^{*}$-seminorm automatically satisfies properties (i) and (ii), above.
} 
is a $\mathrm{C}^{*}$-algebra under the norm

$$
\|a\|_{\infty}:=\sup _{p \in \Gamma} p(a)
$$

and it is $\tau$-dense in $\mathcal{A}$ [100, Proposition 1.11 (4)]. For example, for the locally $\mathrm{C}^{*}$-algebra $C\left(\mathbb{R}^{d}\right)$ of all continuous functions on $\mathbb{R}^{d}$, topologized by the family of seminorms defined by sup-norms over the compact subsets of $\mathbb{R}^{d}$, the corresponding $\mathrm{C}^{*}$-algebra of bounded elements is the algebra $C_{b}\left(\mathbb{R}^{d}\right)$ of bounded continuous functions on $\mathbb{R}^{d}$.

Let $(\Lambda, \preceq)$ be a directed set and $\left\{\mathcal{H}_{\lambda}\right\}_{\lambda \in \Lambda}$ a family of Hilbert spaces such that

$$
\mathcal{H}_{\lambda} \subseteq \mathcal{X}_{\nu}, \quad\langle\cdot, \cdot\rangle_{\lambda}=\left.\langle\cdot, \cdot\rangle_{\nu}\right|_{\mathcal{H}_{\lambda}}
$$

whenever $\lambda, \nu \in \Lambda$ satisfy $\lambda \preceq \nu$. If

$$
i_{\lambda \nu}: \mathcal{H}_{\lambda} \longrightarrow \mathcal{H}_{\nu}, \quad \lambda \preceq \nu
$$

denotes the inclusion of $\mathcal{H}_{\lambda}$ into $\mathcal{H}_{\nu}$, then $\left(\mathcal{H}_{\lambda}, i_{\lambda \nu}\right)$ is an inductive system of Hilbert spaces. The inductive limit vector space

$$
\mathcal{H}:=\lim _{\longrightarrow} \mathcal{H}_{\lambda}=\bigcup_{\lambda \in \Lambda} \mathcal{H}_{\lambda}
$$

when equipped with the inductive limit topology (in other words, the finest topology making the canonical inclusions $i_{\lambda}: \mathcal{H}_{\lambda} \longrightarrow \mathcal{H}, \lambda \in \Lambda$, continuous), is called a locally Hilbert space. Given a family of linear maps $\left\{T_{\lambda}\right\}_{\lambda \in \Lambda}$, where $T_{\lambda} \in \mathcal{L}\left(\mathcal{H}_{\lambda}\right)$, for every $\lambda \in \Lambda$, and such that $\left.T_{\nu}\right|_{\mathcal{H}_{\lambda}}=T_{\lambda}$, whenever $\lambda \preceq \nu$, one can associate a unique continuous linear map

$$
T:=\lim _{\longrightarrow} T_{\lambda}: \mathcal{H} \longrightarrow \mathcal{H},\left.\quad T\right|_{\mathcal{H}_{\lambda}}:=T_{\lambda}, \quad \lambda \in \Lambda .
$$

Define $\mathcal{L}(\mathcal{H})$ as the set of all continuous linear maps of $\mathcal{H}$ into $\mathcal{H}$ such that $T=\underline{\lim } T_{\lambda}$, for some family of operators $\left\{T_{\lambda}\right\}_{\lambda \in \Lambda}$ satisfying $\left.T_{\nu}\right|_{\mathcal{H}_{\lambda}}=T_{\lambda}$, whenever $\lambda \preceq \nu$, where $T_{\lambda} \in \mathcal{L}\left(\mathcal{H}_{\lambda}\right)$. Then, $\mathcal{L}(\mathcal{H})$ becomes an algebra with the natural operations induced by the operator algebras $\mathcal{L}\left(\mathcal{H}_{\lambda}\right), \lambda \in \Lambda$. The involution $*$ on an operator $T$ in $\mathcal{L}(\mathcal{H})$ is defined as the inductive limit operator $T^{*}:=\underline{\lim } T_{\lambda}^{*}$, and the topology on $\mathcal{L}(\mathcal{H})$ is generated by the family $\left\{\left\|T_{\lambda}\right\|_{\lambda}\right\}_{\lambda \in \Lambda}$ of $\mathrm{C}^{*}$-seminorms, where $\|\cdot\|_{\lambda}$ denotes the usual operator norm on $\mathcal{L}\left(\mathcal{H}_{\lambda}\right)$. When equipped with this topology and these algebraic operations, $\mathcal{L}(\mathcal{H})$ becomes a locally $\mathrm{C}^{*}$-algebra [53, Example 7.6 (5), page 106]. By [53, Theorem 8.5 , page 114], every locally $\mathrm{C}^{*}$-algebra $\mathcal{A}$ is $*$-isomorphic to a closed $*$-subalgebra of $\mathcal{L}(\mathcal{H})$, where $\mathcal{H}$ is a locally Hilbert space. A commutative Gelfand-Naimark-type theorem is also available for locally $\mathrm{C}^{*}$-algebras, just as in the 
$\mathrm{C}^{*}$ normed setting (see [53, Theorem 9.3, page 117] and [53, Remarks 9.6, page 119]; see, also, [53, Section 10 - Functional Calculus, page 119]).

By [53, Theorem 10.24, page 133], every locally $\mathrm{C}^{*}$-algebra has complete quotients.

3. The $\mathbf{C}^{*}$-like locally convex $*$-algebras: ${ }^{3}$ Let $(\mathcal{A}, \tau)$ be a locally convex $*$-algebra with identity. A fundamental system of seminorms $\Gamma=\left\{p_{\lambda}\right\}_{\lambda \in \Lambda}$ is called $\mathbf{M}^{*}$-like if, for all given $\lambda \in \Lambda$, there exists $\lambda^{\prime} \in \Lambda$ satisfying the properties

$$
p_{\lambda}(a b) \leq p_{\lambda^{\prime}}(a) p_{\lambda^{\prime}}(b), \quad p_{\lambda}\left(a^{*}\right) \leq p_{\lambda^{\prime}}(a), \quad a, b \in \mathcal{A}
$$

and if, furthermore, $p_{\lambda}(a)^{2} \leq p_{\lambda^{\prime}}\left(a^{*} a\right)$, for every $a \in \mathcal{A}$, then $\Gamma$ is said to be $\mathbf{C}^{*}$ like. Hence, each $p \in \Gamma$ may not necessarily be an $\mathrm{m}^{*}$-seminorm, and neither a $\mathrm{C}^{*}$-seminorm. Also, the norm $p_{\Gamma}: a \longmapsto \sup \left\{p_{\lambda}(a): \lambda \in \Lambda\right\}$ on the $*$-subalgebra

$$
\mathcal{D}_{p_{\Gamma}}:=\left\{a \in \mathcal{A}: \sup \left\{p_{\lambda}(a): \lambda \in \Lambda\right\}<\infty\right\},
$$

which will be called "the $\mathrm{C}^{*}$-algebra of bounded elements of $\mathcal{A}$ ", is generally not welldefined on all of $\mathcal{A}$, and is an $m^{*}$-norm, respectively, a $\mathrm{C}^{*}$-norm whenever $\Gamma$ is $\mathrm{M}^{*}$-like or $\mathrm{C}^{*}$-like, respectively - such a norm is called an unbounded $\mathrm{m}^{*}$-norm, respectively, unbounded $\mathrm{C}^{*}$-norm on $\mathcal{A}$.

A locally convex $*$-algebra is said to be an $\mathbf{M}^{*}$-like (respectively, $\mathbf{C}^{*}$-like) locally convex $*$-algebra if it is complete and if there exists an $\mathrm{M}^{*}$-like (respectively, $\mathrm{C}^{*}$ like) family of seminorms defining the topology of $\mathcal{A}$ in such a way that $\mathcal{D}_{p_{\Gamma}}$ is $\tau$-dense in $\mathcal{A}$. Examples of $\mathrm{C}^{*}$-like locally convex $*$-algebras are:

- The locally $\mathrm{C}^{*}$-algebras.

- The function algebra

$$
L^{\omega}(\mathcal{X}, \mathcal{B}, \mu):=\bigcap_{1 \leq p<+\infty} L^{p}(\mathcal{X}, \mathcal{B}, \mu)
$$

constructed from a finite measure space $(\mathcal{X}, \mathcal{B}, \mu)$ and equipped with the $\mathrm{C}^{*}$-like family

$$
\left\{\frac{1}{\mu(X)^{1 / p}}\|\cdot\|_{p}: 1 \leq p<+\infty\right\}
$$

of norms, where $\|\cdot\|_{p}$ denotes the usual $L^{p}$-norm (see [71, Example 3.3(1)]). In particular, the Arens algebra

$$
L^{\omega}([0,1]):=\bigcap_{1 \leq p<+\infty} L^{p}([0,1]),
$$

defined in [6], is a $\mathrm{C}^{*}$-like locally convex $*$-algebra (see also [53, Definition 2.1(i), page 14]).

\footnotetext{
${ }^{3}$ This definition was taken from the Introduction of [71].
} 
- A noncommutative analog of the previous example: the space

$$
L^{\omega}\left(\tau^{\prime}\right):=\bigcap_{1 \leq p<+\infty} L^{p}\left(\tau^{\prime}\right)
$$

constructed from a faithful finite trace $\tau^{\prime}$ of a von Neumann algebra and equipped with the $\mathrm{C}^{*}$-like family

$$
\left\{\frac{1}{\tau^{\prime}(I)^{1 / p}}\|\cdot\|_{p}: 1 \leq p<+\infty\right\}
$$

of norms, where $\|\cdot\|_{p}$ denotes the $L^{p}$-norm of the Segal space $L^{p}\left(\tau^{\prime}\right)$ with respect to $\tau^{\prime}$ (see [71, Example 3.3(2)]).

- Some particular types of $\mathrm{O}^{*}$-algebras, which may be found in [71, Example 3.4].

4. The GB*-algebras: The following definition may be found in [5], the paper in which $\mathrm{GB}^{*}$-algebras were defined for the first time: let $(\mathcal{A}, \tau)$ be a locally convex $*$-algebra with identity 1 and denote by $\mathcal{B}$ the collection of all closed, bounded, convex and balanced subsets $B$ of $\mathcal{A}$ such that $1 \in B$ and $B^{2} \subseteq B$. Also, denote by $\mathcal{B}^{*}$ the collection of all $B \in \mathcal{B}$ satisfying $B^{*}=B$. For each $B \in \mathcal{B}^{*}$, define $\mathcal{A}[B]$ as the *-algebra generated by $B$ and consider the norm

$$
\|\cdot\|_{B}: x \longmapsto\|x\|_{B}:=\inf \{\lambda>0: x \in \lambda B\}
$$

on $\mathcal{A}[B]$. Then, $\left(\mathcal{A}[B],\|\cdot\|_{B}\right)$ is a normed $*$-algebra, for each $B \in \mathcal{B}^{*}$, and

$$
\mathcal{A}[B]=\{\lambda a: \lambda \in \mathbb{C}, a \in B\} .
$$

If $\mathcal{A}[B]$ is, moreover, complete, for all $B \in \mathcal{B}$, then $\mathcal{A}$ is said to be pseudo-complete (for example, if $\mathcal{A}$ is sequentially complete, then $\mathcal{A}$ is pseudo-complete [4, Proposition 2.6]). A pseudo-complete locally convex $*$-algebra $\mathcal{A}$ is said to be a $\mathbf{G B}^{*}$-algebra if $\mathcal{B}^{*}$ has a greatest element $B_{0}$ and, for all $a \in \mathcal{A}$, the inverse $\left(1+a^{*} a\right)^{-1}$ exists and is a bounded element of $\mathcal{A}$ - an element $a \in \mathcal{A}$ is said to be bounded if, for some non-zero complex number $\lambda$, the set $\left\{(\lambda a)^{n}: n \in \mathbb{N}, n \geq 1\right\}$ is bounded. ${ }^{4}$ In this case, $\mathcal{A}$ is said to be a $\mathrm{GB}^{*}$-algebra over $B_{0}$. Moreover, $\left(\mathcal{A}\left[B_{0}\right],\|\cdot\|_{B_{0}}\right)$ is a $\mathrm{C}^{*}$-algebra with identity [5, Theorem 2.6], $\mathcal{A}\left[B_{0}\right]$ is $\tau$-sequentially dense in $\mathcal{A}$ [15, Theorem (2)] and its norm-topology is finer than the restricted topology $\tau$ (see page 400 of [4]). ${ }^{5}$ Some examples:

\footnotetext{
${ }^{4}$ Note that the definition of pseudo-completeness given in [5] is slightly different from the ones given in $[71$, page 52] and [54, page 171] - these two references ask for completeness of $\mathcal{A}[B]$ only for the elements $B$ of $\mathcal{B}^{*}$. Also, in the definition of $\mathrm{GB}^{*}$-algebras of [71], it is required that the inverses $\left(1+a^{*} a\right)^{-1}$ exist and belong to $\mathcal{A}[B]$, for all $a \in \mathcal{A}$; this condition is slightly different from the one required in [4, Definition 2.5].

${ }^{5}$ For the subject of derivations on GB*-algebras, see [21] (more precisely, the article "Unbounded Derivations of $\mathrm{GB}^{*}$-Algebras"), [123] and [124]. See also [55].
} 
- All $\mathrm{C}^{*}$-like locally convex $*$-algebras are $\mathrm{GB}^{*}$-algebras, by [71, Theorem 2.1].

- $\left[10\right.$, Theorem 3.3]: Let $\left(\mathcal{A}_{0},\|\cdot\|_{0}\right)$ be a unital $\mathrm{C}^{*}$ normed algebra (not necessarily complete) and $\tau$ a locally convex topology on $\mathcal{A}_{0}$ defined by a directed family of seminorms $\left\{p_{\lambda}\right\}_{\lambda \in \Lambda}$. Suppose that:

(i) $\left(\mathcal{A}_{0}, \tau\right)$ is a locally convex $*$-algebra;

(ii) $\tau \subseteq \tau_{0}$, where $\tau_{0}$ is the topology generated by $\|\cdot\|_{0}$, with $\tau$ and $\tau_{0}$ being compatible (this means that every $\|\cdot\|_{0}$-Cauchy net in $\mathcal{A}_{0}$ which $\tau$-converges to 0 must be $\tau_{0}$-convergent to 0 );

(iii) for every $\lambda \in \Lambda$ there exists $\lambda^{\prime} \in \Lambda$ and $c_{\lambda}>0$ such that $p_{\lambda}(a b) \leq$ $c_{\lambda}\|a\|_{0} p_{\lambda^{\prime}}(b)$, for all $a, b \in \mathcal{A}_{0}$.

Furthermore, denote by $\overline{\mathcal{A}_{0}} \tau_{0}$ the $\mathrm{C}^{*}$-algebra completion of $\left(\mathcal{A}_{0},\|\cdot\|_{0}\right)$ and suppose that the unit ball $\mathcal{U}\left({\overline{\mathcal{A}_{0}}}^{\tau}\right):=\left\{a \in{\overline{\mathcal{A}_{0}}}^{\tau_{0}}:\|a\|_{0} \leq 1\right\}$ of ${\overline{\mathcal{A}_{0}}}^{\tau_{0}}$ is $\tau$-closed in the locally convex $\tau$-completion ${\overline{\mathcal{A}_{0}}}^{\tau}$ of $\left(\mathcal{A}_{0}, \tau\right)$. Then, every algebraically symmetric locally convex $*$-algebra $(\mathcal{A}, \tau)$ (in other words, every locally convex *-algebra $(\mathcal{A}, \tau)$ in which the element $1+a^{*} a$ has an inverse in $\mathcal{A}$, for all $\left.a \in \mathcal{A}\right)$ such that ${\overline{\mathcal{A}_{0}}}^{\tau_{0}} \subseteq \mathcal{A} \subseteq{\overline{\mathcal{A}_{0}}}^{\tau}$ is a $\mathrm{GB}^{*}$-algebra over $\mathcal{U}\left({\overline{\mathcal{A}_{0}}}^{\tau_{0}}\right)$.

- Using a definition of $\mathrm{GB}^{*}$-algebras slightly more general ${ }^{6}$ than the one presented above, Dixon proves in [46] that any norm-closed $*$-subalgebra of $\mathcal{L}(\mathcal{H})$ containing the identity operator on $\mathcal{H}$ is a $\mathrm{GB}^{*}$-algebra, if it is equipped with the weak operator topology - see [46, Example (3.3), page 696]. In [46, Definition (7.1)], a set $\mathcal{A}$ of closed operators with dense domains on a Hilbert space $\mathcal{H}$ is called a *-algebra of closed operators on $\mathcal{H}$ if it forms a $*$-algebra under the following operations of addition, multiplication, multiplication by a scalar, and involution:

$$
\begin{gathered}
(A, B) \longmapsto \overline{A+B}, \quad(A, B) \longmapsto \overline{A B}, \\
(\lambda, A) \longmapsto \lambda \bar{A}, \quad A \longmapsto A^{*} .
\end{gathered}
$$

( $A^{*}$ denotes the Hilbert space adjoint of $A$ ) This definition implicitly requires that the sum and the composition of these operators are always closable. If a *-algebra of closed operators $\mathcal{A}$ satisfies the properties that $A \cap \mathcal{L}(\mathcal{H})$ is a $\mathrm{C}^{*}$-algebra and $\left(1+A^{*} A\right)^{-1}$ belongs to $\mathcal{A}$, for all $A \in \mathcal{A}$, then it is called an

\footnotetext{
${ }^{6}$ His definition is made for topological $*$-algebras which are not necessarily locally convex. First, he defines $\mathcal{B}^{\prime}$ to be the collection of all closed and bounded subsets $B$ of $\mathcal{A}$ such that $1 \in B, B^{2} \subseteq B$ and $B=B^{*}$. Then, in [46, page 694], P.G. Dixon defines a $\mathrm{GB}^{*}$-algebra as a topological $*$-algebra such that (i) $\mathcal{B}^{\prime}$ has a greatest element $B_{0}$ which is convex and balanced; (ii) for all $a \in \mathcal{A}$, the inverse $\left(1+a^{*} a\right)^{-1}$ exists and is a bounded element of $\mathcal{A}$; (iii) $\mathcal{A}\left[B_{0}\right]$ is complete. His definition of $\mathrm{GB}^{*}$-algebras generalizes the original definition given by G.R. Allan, in the sense that every GB*-algebra in the sense of Allan [5] is a GB*-algebra in the sense of Dixon [46].
} 
extended $\mathbf{C}^{*}$-algebra on $\mathcal{H}$ (the operator $\left(1+A^{*} A\right)^{-1}$ already belongs to $\mathcal{L}(\mathcal{H})$, because $A$ is a closed and densely defined operator on $\mathcal{H})$. This algebra $\mathcal{A}$ is said to have a common dense domain $\mathcal{K}$ if

$$
\mathcal{K}:=\bigcap_{A \in \mathcal{A}} \operatorname{Dom} A
$$

is dense in $\mathcal{H}$. The main theorem [46, Theorem 7.13] of the paper states that a $*$-algebra $\mathcal{A}$ can be equipped with a locally convex GB$^{*}$-topology if, and only if, it is algebraically isomorphic to an extended $\mathrm{C}^{*}$-algebra with common dense domain.

5. Von Neumann Algebras: There are several choices of topologies for $\mathcal{L}(\mathcal{H})$, besides the topology induced by the usual operator norm: the strong, $\sigma$-strong (also called "ultra-strong"), weak and $\sigma$-weak (also called "ultra-weak") operator topologies are all Hausdorff locally convex topologies on $\mathcal{L}(\mathcal{H})$, none of which is metrizable (in other words, they cannot be defined by a countable family of seminorms), unless $\mathcal{H}$ is finite-dimensional [22, I.3.1.4, page 14]. For a matter of convenience, the strong and the weak operator topologies shall be called "strong topology" and "weak topology", respectively, in this text - one should not confuse the weak and the strong operator topologies just mentioned with the weak and the strong topologies mentioned in Chapter 1, in the context of locally convex spaces (see [22, I.3.1.1, page 13]). Also, if $\mathcal{H}$ is infinite dimensional, $\mathcal{L}(\mathcal{H})$ is not complete when equipped with the strong or the weak topology, but it is complete with respect to the $\sigma$-strong topology - see [22, I.3.2.2, page 15]. These choices of possible topologies present different levels of compatibility with the algebraic operations: the multiplication is separately continuous in all of the topologies mentioned above, but is not jointly continuous in any of them, if $\mathcal{H}$ is infinite-dimensional - see [22, I.3.2.1, page 14], [30, Propositions 2.4.1 and 2.4.2] and [53, pages $9,10,11,12]$. The adjoint operation is weakly and $\sigma$-weakly continuous ([93, page 126], [30, Proposition 2.4.2, page 68]), but it is not continuous with respect to the strong topology [22, Example I.3.1.6, page 14], nor to the $\sigma$-strong topology [30, Proposition 2.4.1, page 67]. However, it is possible to enlarge the usual families defining the strong and the $\sigma$-strong topologies in order to make the adjoint operation continuous, giving rise to the strong* and the $\sigma$-strong* topologies, as is shown in [22, Example I.3.1.6, page 14] or [30, page 69]; see also [53, Theorem 3.7, page 32], for a statement in a more general framework.

A von Neumann algebra $\mathcal{A}$ is defined as a *-subalgebra of $\mathcal{L}(\mathcal{H})$ such that $\mathcal{A}=\mathcal{A}^{\prime \prime}$, where $\mathcal{H}$ is some Hilbert space, $\mathcal{A}^{\prime}:=\{B \in \mathcal{L}(\mathcal{H}): A B=B A$, for all $A \in \mathcal{A}\}$ and $\mathcal{A}^{\prime \prime}:=\left(\mathcal{A}^{\prime}\right)^{\prime}$ - see [22, page 221], [44]. ${ }^{7}$ A remarkable aspect of von Neumann algebras

\footnotetext{
${ }^{7}$ The reference [93, page 116] defines a von Neumann algebra as a $*$-subalgebra of $\mathcal{L}(\mathcal{H})$ which is closed with respect to the strong topology, when it is relativized to $\mathcal{A}$.
} 
is that, despite the purely algebraic definition, it is possible to characterize them via the topologies just defined, at least when they are non-degenerate $*$-subalgebras ${ }^{8}$ of $\mathcal{L}(\mathcal{H})$. More precisely: ${ }^{9}$

The Bicommutant Theorem: Let $\mathcal{A}$ be a non-degenerate $*$-subalgebra of $\mathcal{L}(\mathcal{H})$. Then, $\mathcal{A}$ is a von Neumann algebra if, and only if, it is closed with respect to any of the topologies defined above.

See also references [114] and [116] for expositions on the topic of $\mathrm{W}^{*}$-algebras.

A particular class of examples of locally convex $*$-algebras $(\mathcal{A}, \Gamma)$ which have complete quotients is given by the following theorem:

Theorem 3.2 (Complete Quotients): Let $\mathcal{A}$ be a locally convex *-algebra and $\Gamma$ be a fundamental system of $*$-seminorms for $\mathcal{A}$. Suppose that

1. there exists a dense $*$-subalgebra $\mathcal{A}_{0}$ of $\mathcal{A}$ and a $C^{*}$-norm $\|\cdot\|_{0}: \mathcal{A}_{0} \longrightarrow[0,+\infty)$ on $\mathcal{A}_{0}$ such that the topology $\tau_{0}$ on $\mathcal{A}_{0}$ induced by the norm $\|\cdot\|_{0}$ is finer than the topology $\tau$ induced by $\Gamma\left(\tau \subseteq \tau_{0}\right)$ and

2. $\left(\mathcal{A}_{0},\|\cdot\|_{0}\right)$ is a $C^{*}$-algebra.

Then, if the seminorms' kernels $N_{p}$ are ideals in $\mathcal{A},{ }^{10} \mathcal{A} / N_{p}$ is a Banach space when equipped with the usual norm $\|\cdot\|_{p}:[a]_{p} \longmapsto\left\|[a]_{p}\right\|_{p}:=p(a)$, for all $p \in \Gamma$. Moreover, there is another norm $\|\cdot\|_{p}^{\prime \prime}$ on $\mathcal{A} / N_{p}$ which is equivalent to $\|\cdot\|_{p}$ possessing the property that $\left(\mathcal{A} / N_{p},\|\cdot\|_{p}^{\prime \prime}\right)$ is Banach *-algebra.

Proof of Theorem 3.2: Fix $p \in \Gamma$. Then, since $N_{p}$ is an ideal in $\mathcal{A}$, the map

$$
\left([a]_{p},[b]_{p}\right) \longmapsto[a]_{p}[b]_{p}:=[a b]_{p}
$$

on each $\mathcal{A} / N_{p}$ is a well-defined product which turns it into an algebra - due to an aesthetic reason, the subscript " $p$ " of the equivalence classes $[\cdot]_{p}$ in the next paragraphs will be suppressed. The map $\|\cdot\|_{p}:[a]_{p} \longmapsto p(a)$ on $\mathcal{A} / N_{p}$ defines a $*$-norm on $\mathcal{A} / N_{p}$, but not necessarily a submultiplicative one. The first step will be to prove that a new submultiplicative $*$-norm $\|\cdot\|_{p}^{\prime \prime}$, which is equivalent to $\|\cdot\|_{p}$, may be defined on $\mathcal{A} / N_{p}$.

\footnotetext{
${ }^{8} \mathrm{~A} *$-subalgebra $\mathcal{A}$ of $\mathcal{L}(\mathcal{H})$ is said to be non-degenerate if $\overline{\operatorname{span}_{\mathbb{C}}\{A x: A \in \mathcal{A}, x \in \mathcal{H}\}}=\mathcal{H}$.

${ }^{9}$ See [30, Theorem 2.4.11, page 72], [22, Theorem I.9.1.1, page 47] and [93, Theorem 4.1.5, page 116].

${ }^{10}$ Note that, since $\mathcal{A}$ is not assumed to be an m-convex algebra, the kernels $N_{p}$ may not be ideals; they are only vector subspaces of $\mathcal{A}$ which are left invariant by the involution. However, if $\Gamma$ is assumed to be a fundamental system of $\mathrm{m}^{*}$-seminorms for $\mathcal{A}$ - and, so, $\mathcal{A}$ would be an $\mathrm{m}^{*}$-convex algebra - this condition is automatically satisfied.
} 
By the Uniform Boundedness Theorem for separately continuous bilinear maps applied to $\left(\overline{\mathcal{A} / N_{p}},\|\cdot\|_{p}\right)$, there exists $M_{p} \geq 1$ such that $\|[a][b]\|_{p} \leq M_{p}\|[a]\|_{p}\|[b]\|_{p}$, for all $a, b \in \mathcal{A}$. Since

$$
\|\lambda[a]+[a][b]\|_{p} \leq|\lambda|\|[a]\|_{p}+M_{p}\|[a]\|_{p}\|[b]\|_{p}, \quad a, b \in \mathcal{A},|\lambda|+\|[b]\|_{p} \leq 1,
$$

it follows that, for each fixed $a \in \mathcal{A}$,

$$
\|[a]\|_{p}^{\prime}:=\sup \left\{\|\lambda[a]+[a][b]\|_{p}: \lambda \in \mathbb{C}, b \in \mathcal{A},|\lambda|+\|[b]\|_{p} \leq 1\right\} \leq\left(M_{p}+1\right)\|[a]\|_{p} .
$$

Therefore, $\|\cdot\|_{p}^{\prime}$ is a well-defined norm on $\mathcal{A} / N_{p}$. Moreover, one easily sees that $\|[a]\|_{p} \leq$ $\|[a]\|_{p}^{\prime}$, for all $a \in \mathcal{A}$. Thus, $\|\cdot\|_{p}$ and $\|\cdot\|_{p}^{\prime}$ are equivalent norms on $\mathcal{A} / N_{p}$. The norm $\|\cdot\|_{p}^{\prime}$ is submultiplicative, by $[98$, Proposition 1.1 .9 , page 15$]$ : in fact, if $[c]=0$, then $\|[a][c]\|_{p}^{\prime}=0=\|[a]\|_{p}^{\prime}\|[c]\|_{p}^{\prime}$. If $[c] \neq 0$, then

$$
\begin{gathered}
\|[a][c]\|_{p}^{\prime}=\sup \left\{\|\lambda[a][c]+[a][c][b]\|_{p}:|\lambda|+\|[b]\|_{p} \leq 1\right\} \\
\leq \sup \left\{\|\lambda[a][c]+[a][c][b]\|_{p}:\|\lambda[c]+[c][b]\|_{p} \leq\|c\|_{p}^{\prime}\right\} \\
=\|[c]\|_{p}^{\prime}\left(\sup \left\{\frac{\|\lambda[a][c]+[a][c][b]\|_{p}}{\|[c]\|_{p}^{\prime}}: \frac{\|\lambda[c]+[c][b]\|_{p}}{\|[c]\|_{p}^{\prime}} \leq 1\right\}\right) \\
\leq\|[c]\|_{p}^{\prime}\left(\sup \left\{\|[a][d]\|_{p}:\|[d]\|_{p} \leq 1\right\}\right) \leq\|[a]\|_{p}^{\prime}\|[c]\|_{p}^{\prime},
\end{gathered}
$$

so $\|\cdot\|_{p}^{\prime}$ is indeed submultiplicative. The fact that the induced involution on $\mathcal{A} / N_{p}$ is $\|\cdot\|_{p}$-isometric implies the equality

$$
\begin{gathered}
\left\|[a]^{*}\right\|_{p}^{\prime}=\sup \left\{\left\|\bar{\lambda}\left[a^{*}\right]+\left[a^{*} b^{*}\right]\right\|_{p}:|\lambda|+\|[b]\|_{p} \leq 1, \lambda \in \mathbb{C}, b \in \mathcal{A}\right\} \\
=\sup \left\{\left\|(\lambda[a]+[b][a])^{*}\right\|_{p}:|\lambda|+\|[b]\|_{p} \leq 1, \lambda \in \mathbb{C}, b \in \mathcal{A}\right\} \\
=\sup \left\{\|\lambda[a]+[b][a]\|_{p}:|\lambda|+\|[b]\|_{p} \leq 1, \lambda \in \mathbb{C}, b \in \mathcal{A}\right\}, \quad a \in \mathcal{A} .
\end{gathered}
$$

Hence, defining

$$
\|[a]\|_{p}^{\prime \prime}:=\max \left\{\|[a]\|_{p}^{\prime},\left\|[a]^{*}\right\|_{p}^{\prime}\right\}, \quad a \in \mathcal{A}
$$

gives a submultiplicative norm on $\mathcal{A} / N_{p}$, equivalent to $\|\cdot\|_{p}$, for which the induced involution is isometric. Finally, this shows that the Banach space completions of $\mathcal{A} / N_{p}$ with respect to $\|\cdot\|_{p}$ and $\|\cdot\|_{p}^{\prime \prime}$ are isomorphic (as Banach spaces), but the completion $\overline{\mathcal{A} / N_{p}}{ }^{\prime \prime}$ with respect to the latter norm gives rise to a Banach $*$-algebra.

Now, the set $I_{p}:=\mathcal{A}_{0} \cap N_{p}=\left\{a \in \mathcal{A}_{0}: p(a)=0\right\}$ is a closed $*$-ideal in $\left(\mathcal{A}_{0},\|\cdot\|_{0}\right)$ so that $\left(\mathcal{A}_{0} / I_{p},\|\cdot\|_{I_{p}}\right)$, with

$$
\left\|[a]_{I_{p}}\right\|_{I_{p}}:=\inf _{b \in I_{p}}\|a+b\|_{0}, \quad[a]_{I_{p}} \in \mathcal{A}_{0} / I_{p}
$$


being the quotient norm on $\mathcal{A}_{0} / I_{p}$, is a $\mathrm{C}^{*}$-algebra ([93, Theorem 3.1.4, page 80]).

The *-homomorphism

$$
\theta: \mathcal{A}_{0} / I_{p} \longrightarrow \mathcal{A} / N_{p}:[a]_{I_{p}} \longmapsto[a]
$$

is well-defined, injective and is $\left(\|\cdot\|_{I_{p}},\|\cdot\|_{p}^{\prime \prime}\right)$-continuous: in fact, there exists $C_{p}>0$ such that, for each fixed $a \in \mathcal{A}_{0}$ and $n \in I_{p}$,

$$
\left\|\theta\left([a]_{I_{p}}\right)\right\|_{p}=\|[a]\|_{p}=p(a)=p(a+n) \leq C_{p}\|a+n\|_{0},
$$

because $\tau \subseteq \tau_{0}$. Therefore,

$$
\left\|\theta\left([a]_{I_{p}}\right)\right\|_{p} \leq C_{p} \inf \left\{\|a+n\|_{0}: n \in I_{p}\right\}=C_{p}\left\|[a]_{I_{p}}\right\|_{I_{p}}
$$

and, since $\|\cdot\|_{p}$ and $\|\cdot\|_{p}^{\prime \prime}$ are equivalent norms, this establishes the desired continuity. Because $\mathcal{A}_{0}$ is dense in $\mathcal{A}, \theta$ also has dense range. Now, by [120, Proposition 5.3, page $22]^{11}$ applied to the $\mathrm{C}^{*}$-algebra $\left(\mathcal{A}_{0} / I_{p},\|\cdot\|_{I_{p}}\right)$ and the Banach $*$-algebra $\left({\overline{\mathcal{A} / N_{p}}}^{\prime \prime},\|\cdot\|_{p}^{\prime \prime}\right)$, the inequality

$$
\left\|\theta\left([a]_{I_{p}}\right)\right\|_{p}^{\prime \prime} \geq\left\|[a]_{I_{p}}\right\|_{I_{p}}, \quad a \in \mathcal{A}_{0},
$$

holds. Hence, since $\mathcal{A}_{0} / I_{p}$ is complete, $\theta\left[\mathcal{A}_{0} / I_{p}\right]$ must also be a $\|\cdot\|_{p}^{\prime \prime}$-closed $*$-subalgebra of $\mathcal{A} / N_{p}$, from which surjectivity of $\theta$ follows. This implies $\mathcal{A} / N_{p}$ is $\|\cdot\|_{p}^{\prime \prime}$-complete, so it is also $\|\cdot\|_{p}$-complete, as claimed.

Note that, because the Banach *-algebra $\left(\mathcal{A} / N_{p},\|\cdot\|_{p}^{\prime \prime}\right)$ is $*$-isomorphic to the $\mathrm{C}^{*}$ algebra $\left(\mathcal{A}_{0} / I_{p},\|\cdot\|_{I_{p}}\right)$, for all $p \in \Gamma$, it follows that the $*$-algebras $\mathcal{A} / N_{p}$ themselves may be equipped with a $\mathrm{C}^{*}$ norm $n_{p}$ defined by

$$
n_{p}([a]):=\left\|\theta^{-1}([a])\right\|_{I_{p}}, \quad[a] \in \mathcal{A} / N_{p},
$$

which is equivalent to $\|\cdot\|_{p}^{\prime \prime}$. Therefore, $\left(\mathcal{A} / N_{p}, n_{p}(\cdot)\right)$ is a $\mathrm{C}^{*}$-algebra, for every $p \in \Gamma$.

Corollary 3.3: Suppose $(\mathcal{A}, \Gamma)$ is a locally convex $*$-algebra, with $\Gamma$ being a fundamental system of $*$-seminorms for $\mathcal{A}$. Then, $N_{p}$ is an ideal in $\mathcal{A}$, for all $p \in \Gamma$ if, and only if, $\mathcal{A}$ is an $m^{*}$-convex algebra.

Proof of Corollary 3.3: Suppose $N_{p}$ is an ideal in $\mathcal{A}$, for all $p \in \Gamma$. Just like in the proof of Theorem 3.2 define, for each $p \in \Gamma$, the seminorm

$$
p^{\prime}(a):=\sup \left\{\left\|\lambda[a]_{p}+[a]_{p}[b]_{p}\right\|_{p}: \lambda \in \mathbb{C}, b \in \mathcal{A},|\lambda|+\left\|[b]_{p}\right\|_{p} \leq 1\right\}, \quad a \in \mathcal{A},
$$

\footnotetext{
${ }^{11}\left[120\right.$, Proposition 5.3, page 22]: Let $\mathcal{A}$ be a $\mathrm{C}^{*}$-algebra and $\mathcal{B}$ a Banach $*$-algebra. If $\pi$ is a $*$-isomorphism of $\mathcal{A}$ into $\mathcal{B}$, then

$$
\|\pi(a)\|_{\mathcal{B}} \geq\|a\|_{\mathcal{A}}, \quad a \in \mathcal{A}
$$
}


and the seminorm

$$
p^{\prime \prime}(a):=\max \left\{p^{\prime}(a), p^{\prime}\left(a^{*}\right)\right\}, \quad a \in \mathcal{A} .
$$

Then, $\Gamma^{\prime \prime}:=\left\{p^{\prime \prime}: p \in \Gamma\right\}$ is family of submultiplicative $*$-seminorms which generates the same topology as $\Gamma$, so $\mathcal{A}$ is an $\mathrm{m}^{*}$-convex algebra. The converse is immediate.

Corollary 3.4: Suppose $\mathcal{A}$ is a locally convex *-algebra satisfying hypotheses 1. and 2. of Theorem 3.2. If $\mathcal{A}$ is also an Arens-Michael *-algebra (equivalently, a complete $m^{*}$-convex algebra), then it is actually a locally $C^{*}$-algebra. Therefore, the only complete $G B^{*}$-algebras which are $m^{*}$-convex algebras are the locally $C^{*}$-algebras.

Proof of Corollary 3.4: Suppose $\mathcal{A}$ is a complete locally convex $*$-algebra satisfying hypotheses 1 . and 2 . of Theorem 3.2 which is also an $\mathrm{m}^{*}$-convex algebra, with $\Gamma$ being a fundamental system of $\mathrm{m}^{*}$-seminorms for $\mathcal{A}$. By the proof of Theorem 3.2, the seminorms defined by $\tilde{n}_{p}: a \longmapsto n_{p}\left([a]_{p}\right)$ are actually $\mathrm{C}^{*}$-seminorms on $\mathcal{A}$, and the family

$$
\tilde{\Gamma}:=\left\{\tilde{n}_{p}\right\}_{p \in \Gamma}
$$

generates the same topology as $\Gamma$. Therefore, $(\mathcal{A}, \tilde{\Gamma})$ is a locally $\mathrm{C}^{*}$-algebra. The converse is immediate, by the definition and the properties of a locally $\mathrm{C}^{*}$-algebra.

\section{Exponentiation of Complete Locally Convex Algebras}

Now, it will be shown that, once a Lie algebra of operators on a locally convex algebra $\mathcal{A}$ (or on a locally convex *-algebra $\mathcal{A}$ ) is exponentiated, there is an automatic compatibility of the group representation $V: G \longrightarrow \mathcal{L}(\mathcal{A})$ obtained with the additional algebraic structure, and not only with the vector space structure. To this end, an idea which appears in the proof of [29, Theorem 1] was borrowed and adapted to the present context.

Lemma 3.5 (Automatic Compatibility with the Additional Algebraic Operations): Let $(\mathcal{A}, \Gamma)$ be a locally convex algebra (respectively, locally convex $*$-algebra), $\mathcal{D} \subseteq \mathcal{A}$ a dense subalgebra (respectively, *-subalgebra) and $\mathcal{L} \subseteq \operatorname{End}(\mathcal{D})$ be an exponentiable Lie algebra of derivations (respectively, *-derivations) to a strongly continuous locally equicontinuous representation $g \longmapsto V(g)$ of a Lie group. Then, each one-parameter group $V(t, \bar{\delta}): t \longmapsto V(\exp t \delta), \delta \in \mathcal{L}$, of continuous linear operators is actually a representation by automorphisms (respectively, *-automorphisms).

Proof of Lemma 3.5: First, assume that $\mathcal{A}$ is only a locally convex algebra. Fix $\delta \in \mathcal{L}$ and $t \in \mathbb{R}$. The operator $V(t, \bar{\delta})$ will be abbreviated to $V_{t}$, to facilitate the reading process. To prove $V_{t}$ is an algebra automorphism, the only thing that remains to be shown is that it preserves the multiplication of $\mathcal{A}$. Define

$$
F: s \longmapsto V_{-s}\left(V_{s}(c) V_{s}(d)\right),
$$


where $c, d$ are fixed elements of Dom $\bar{\delta}$. Since the multiplication operation on $\mathcal{A}$ is separately continuous, the linear map from Dom $\bar{\delta}$ to $\mathcal{A}$ defined by

$$
L_{a}: b \longmapsto a b, \quad a \in \mathcal{A},
$$

is linear and continuous, when Dom $\bar{\delta}$ is equipped with the topology defined by the family of seminorms $\{a \longmapsto p(a)+p(\bar{\delta}(a)): p \in \Gamma\}$. Moreover, since

$$
\frac{V_{s+h}(a) b-V_{s}(a) b}{h}=V_{s}\left(\frac{V_{h}(a)-(a)}{h}\right) b, \quad s \in \mathbb{R}, h \in \mathbb{R} \backslash\{0\}, a, b \in \operatorname{Dom} \bar{\delta},
$$

the strong derivative of $s \longmapsto L_{V(s)(a)}$, for each fixed $a \in \operatorname{Dom} \bar{\delta}$, is

$$
s \longmapsto L_{V(s)(\bar{\delta}(a))},
$$

which maps $\mathbb{R}$ to the space of continuous linear maps from Dom $\bar{\delta}$ to $\mathcal{A}$.

The function $s \longmapsto V_{s}$ also maps $\mathbb{R}$ to the space of continuous linear maps from Dom $\bar{\delta}$ to $\mathcal{A}$ in a strongly differentiable way, with $V_{t} \circ \bar{\delta}$ being its strong derivative at $s=t$. Therefore, applying [74, Theorem A.1, page 440] to $F$, twice, gives

$$
\begin{gathered}
F^{\prime}(t)=-V_{-t} \bar{\delta}\left(V_{t}(c) V_{t}(d)\right)+V_{-t}\left(V_{t}(\bar{\delta}(c)) V_{t}(d)+V_{t}(c) V_{t}(\bar{\delta}(d))\right) \\
=-V_{-t}\left(\bar{\delta}\left(V_{t}(c)\right) V_{t}(d)+V_{t}(c) \bar{\delta}\left(V_{t}(d)\right)\right) \\
+V_{-t}\left(\bar{\delta}\left(V_{t}(c)\right) V_{t}(d)+V_{t}(c) \bar{\delta}\left(V_{t}(d)\right)\right)=0 .
\end{gathered}
$$

Since $F(0)=c d$, a quick application of the Hahn-Banach Theorem gives

$$
V_{-t}\left(V_{t}(c) V_{t}(d)\right)=F(t)=F(0)=c d,
$$

so the arbitrariness of $t, c$ and $d$, together with denseness of Dom $\bar{\delta}$ in $\mathcal{A}$, prove that $V_{t}$ is an algebra homomorphism of $\mathcal{A}$.

Now, suppose $\mathcal{A}$ is a locally convex $*$-algebra. Defining the function

$$
G: s \longmapsto V_{-s}\left(V_{s}(c)^{*}\right),
$$

where $c$ is a fixed element of Dom $\bar{\delta}$, and applying a similar reasoning, one obtains that $V_{t}$ preserves the (continuous) involution operation, for each $t \in \mathbb{R}$.

Remark 3.5.1: It should be mentioned that the proof above, although simple, has some subtleties. The precise specification of the locally convex spaces involved in order to apply [74, Theorem A.1, page 440] is one of them. The other one is that the usual formula for the derivative of the product of two functions defined on $\mathbb{R}$ had to be derived via an alternative approach: since the multiplication of $\mathcal{A}$ is not necessarily jointly continuous, 
the usual proof of this formula (as it is done in many Elementary Calculus books - probably in most of them) may not be adapted to this context.

Finally, some applications of the exponentiation theorems of Chapter 2 may be given.

Definition 3.6: Let $\mathcal{A}$ be a locally convex $*$-algebra and $\mathcal{D} \subseteq \mathcal{A}$ a $*$-subalgebra. Suppose $(\mathcal{D}, \mathfrak{g}, \eta)$ is a representation of $\mathfrak{g}$ by closed linear operators on $\mathcal{A}$, in the sense of the definition made in the last chapter. Suppose, also, that every element of Ran $\eta$ is a *-derivation$a *$-derivation $\delta$ is a linear, *-preserving map defined on a $*$-subalgebra of $\mathcal{A}$ which satisfies the Leibniz rule: $\delta(a b)=\delta(a) b+a \delta(b), a, b \in$ Dom $\delta$. Then, $(\mathcal{D}, \mathfrak{g}, \eta)$ is called a representation of $\mathfrak{g}$ by closed $*$-derivations on $\mathcal{A}$. Now, whenever $(\mathcal{D}, \mathfrak{g}, \eta)$ exponentiates to a Lie group strongly continuous locally equicontinuous representation $\alpha: G \longrightarrow \mathcal{L}(\mathcal{A})$ (in the context of algebras the letter " $\alpha$ ", instead of " $V$ ", will be used), it is a consequence of Lemma 3.5 that each one-parameter group $t \longmapsto \alpha(\exp t X), X \in \mathfrak{g}$, is actually implemented by *-automorphisms. In other words, every operator $\alpha(\exp t X)$ is a *-automorphism on $\mathcal{A}{ }^{12}$ An analogous definition can be done if $\mathcal{A}$ is just a locally convex algebra, substituting the words "*-subalgebra" by "algebra", "*-derivation" by "derivation" and "*-automorphism" by "automorphism".

Combining Theorem 2.16 with Lemma 3.5 one can obtain the following exponentiation theorem for complete locally convex $*$-algebras:

Theorem 3.7 (Exponentiation - Complete Locally Convex Algebras): Let $\mathcal{A}$ be a complete locally convex algebra, $\mathfrak{g}$ a real finite-dimensional Lie algebra with an ordered basis $\mathcal{B}:=\left(X_{k}\right)_{1 \leq k \leq d}$ and $\left(\mathcal{A}_{\infty}, \mathfrak{g}, \eta\right)$ a representation of $\mathfrak{g}$ by closed derivations, with

$$
\mathcal{A}_{\infty}:=\bigcap_{n=1}^{+\infty} \bigcap\left\{\operatorname{Dom}\left[\delta_{i_{1}} \ldots \delta_{i_{k}} \ldots \delta_{i_{n}}\right]: 1 \leq k \leq n, 1 \leq i_{k} \leq d\right\},
$$

where $\delta_{X}:=\eta(X), \delta_{k}:=\eta\left(X_{k}\right), 1 \leq k \leq d$. Suppose that the operators $\left\{\delta_{k}\right\}_{1 \leq k \leq d}$ are $\Gamma$-conservative. Suppose, also, that

$$
H_{m}:=\sum_{\alpha ;|\alpha| \leq m} c_{\alpha} \eta_{\mathcal{A}_{\infty}}\left(X_{1}\right)^{\alpha_{1}} \ldots \eta_{\mathcal{A}_{\infty}}\left(X_{k}\right)^{\alpha_{k}} \ldots \eta_{\mathcal{A}_{\infty}}\left(X_{d}\right)^{\alpha_{d}}
$$

is an element of $\mathfrak{U}\left(\eta_{\mathcal{A}_{\infty}}[\mathfrak{g}]\right)_{\mathbb{C}}$ of order $m \geq 2$ which is strongly elliptic and that $-H_{m}$ is $\Gamma$-dissipative. Then, the following are equivalent:

1. $\left(\mathcal{A}_{\infty}, \mathfrak{g}, \eta\right)$ exponentiates to a strongly continuous locally equicontinuous representation $\alpha: G \longrightarrow \mathcal{L}(\mathcal{A})$ by automorphisms of a simply connected Lie group $G$, having

\footnotetext{
${ }^{12} \mathrm{~A} *$-automorphism is a continuous $*$-preserving algebraic isomorphism of $\mathcal{A}$ onto $\mathcal{A}$ which possesses a continuous inverse.
} 
$\mathfrak{g}$ as its Lie algebra, such that $d \alpha(X)=\overline{\left.\eta(X)\right|_{\mathcal{A}_{\infty}}}$ is the generator of a $\Gamma$-isometrically equicontinuous one-parameter group, for all $X \in \mathfrak{g}$;

2. $-H_{m}$ is an infinitesimal pregenerator of a $\Gamma$-contractively equicontinuous semigroup $t \longmapsto S(t)$ satisfying $S(t)[\mathcal{A}] \subseteq \mathcal{A}_{\infty}$, for all $t \in(0,1]$; moreover, for each $p \in \Gamma$ and $n \in \mathbb{N}$ satisfying $0<n \leq m-1$, there exists $C_{p, n}>0$ for which the estimates

$$
\rho_{p, n}(S(t) a) \leq C_{p, n} t^{-\frac{n}{m}} p(a)
$$

are verified for all $t \in(0,1]$ and $a \in \mathcal{A}$.

If $\mathrm{H}_{2}=\Delta:=\sum_{k=1}^{d} \delta_{k}^{2}$, then the $\Gamma$-dissipativity hypothesis on $\mathrm{H}_{2}$ is superfluous, by the usual arguments. Also, if one of the above conditions is fulfilled, then $\eta$ has a dense set of projective analytic vectors.

Theorem 3.7 remains valid if $\mathcal{A}$ is a complete locally convex $*$-algebra and $\left(\mathcal{A}_{\infty}, \mathfrak{g}, \eta\right)$ is a representation of $\mathfrak{g}$ by closed $*$-derivations, substituting the word "automorphisms" by "*-automorphisms".

\section{Exponentiation of Locally $\mathrm{C}^{*}$-Algebras}

In the special case where $\mathcal{A}$ is a locally $\mathrm{C}^{*}$-algebra, an improvement of Theorem 3.7 may be obtained, at least for the case in which the operator dominating the representation is the negative of the Laplacian. Theorem 3.8, below, is in perfect analogy with the $\mathrm{C}^{*}$ case (see [26, Corollary 2]), the only difference being that the result, below, is proved for arbitrary dense core domains. In order for the proof to work, a theorem from [26] must be used:

Dissipativity of the Laplacian Implies Conservativity of the $*$-Derivations [26, Theorem 1]: Let $\left\{\delta_{k}\right\}_{1 \leq k \leq d}$ be a finite sequence of $*$-derivations on a $C^{*}$-algebra $\mathcal{A}$ such that the Laplacian $\Delta:=\sum_{k=1}^{d} \delta_{k}^{2}$ is densely defined on Dom $\Delta:=\cap_{k=1}^{d}$ Dom $\delta_{k}^{2}$. The following two conditions are equivalent:

1. The operator $\Delta$ is dissipative.

2. The derivations $\left\{\delta_{k}\right\}_{1 \leq k \leq d}$ are conservative on Dom $\Delta$.

Theorem 3.8 (Exponentiation - Locally $\mathbf{C}^{*}$-Algebras, I): Let $\mathcal{A}$ be a locally $C^{*}$-algebra, $\mathcal{D} \subseteq \mathcal{A}$ a dense $*$-subalgebra, $\mathfrak{g}$ a real finite-dimensional Lie algebra with an ordered basis $\mathcal{B}:=\left(X_{k}\right)_{1 \leq k \leq d},(\mathcal{D}, \mathfrak{g}, \eta)$ a representation of $\mathfrak{g}$ by closed $*$-derivations with $\delta_{X}:=\eta(X), \delta_{k}:=\eta\left(X_{k}\right), 1 \leq k \leq d$, and $\Delta:=\sum_{k=1}^{d} \delta_{k}^{2}$. Assume that the following hypotheses are valid: 
$\Delta$ is an infinitesimal pregenerator of an equicontinuous semigroup $t \longmapsto S(t)$ satisfying $S(t)[\mathcal{A}] \subseteq \mathcal{D}$, for all $t \in(0,1]$ and, being a pregenerator of an equicontinuous semigroup, let $\Gamma_{2}$ be a fundamental system of seminorms for $\mathcal{A}$ with respect to which the operator $\Delta$ has the (KIP), is $\Gamma_{2}$-dissipative and $S$ is $\Gamma_{2}$-contractively equicontinuous; also, suppose that, for every $p \in \Gamma_{2}$, there exists $C_{p}>0$ for which the estimates

$$
\rho_{p, 1}(S(t) a) \leq C_{p} t^{-\frac{1}{2}} p(a)
$$

are verified, for all $t \in(0,1]$ and $a \in \mathcal{A}$.

Then, $(\mathcal{D}, \mathfrak{g}, \eta)$ exponentiates to a strongly continuous locally equicontinuous representation $\alpha: G \longrightarrow \mathcal{L}(\mathcal{A})$ by $*$-automorphisms of a simply connected Lie group $G$, having $\mathfrak{g}$ as its Lie algebra, such that $d \alpha(X)=\overline{\left.\eta(X)\right|_{\mathcal{A}_{\infty}}}$ is the generator of a $\Gamma_{2}$-isometrically equicontinuous one-parameter group, for all $X \in \mathfrak{g}$.

Proof of Theorem 3.8: The only thing needed to prove is that the $*$-derivations $\eta(X), X \in \mathfrak{g}$, are all $\Gamma_{2}$-conservative, because then, Theorem 2.12 becomes available. In order to obtain this result, the reference [26, Theorem 1], above, will be used.

As in the beginning of the proof of Theorem 2.12, the estimates

$$
\rho_{p, 1}(a) \leq \epsilon p(\Delta(a))+\frac{E_{p}}{\epsilon} p(a)
$$

are valid for every $p \in \Gamma_{2}, 0<\epsilon \leq 1$ and $a \in \mathcal{D}$, which shows that each of the $*$-derivations $\left.\delta_{k}\right|_{\mathcal{D}}$ possesses the (KIP) with respect to $\Gamma_{2}$. By the triangle inequality, all $*$-derivations $\left.\delta_{X}\right|_{\mathcal{D}}, X \in \mathfrak{g}$, have the $(\mathrm{KIP})$ with respect to $\Gamma_{2}$. Hence, one can define the induced $*$-derivations

$$
\delta_{x, p}:[a]_{p} \longmapsto\left[\delta_{X}(a)\right]_{p}, \quad[a]_{p} \in \pi_{p}[\mathcal{D}],
$$

for all $p \in \Gamma_{2}$. The induced operator $\left(\left.\Delta\right|_{\mathcal{D}}\right)_{p}=\sum_{k=1}^{d} \delta_{k, p}^{2}$ is dissipative on $\cap_{k=1}^{d}$ Dom $\delta_{k, p}^{2}=$ $\pi_{p}[\mathcal{D}]$, so $[26$, Theorem 1] mentioned above says that each induced $*$-derivation

$$
\delta_{k, p}:[a]_{p} \longmapsto\left[\delta_{k}(a)\right]_{p}, \quad 1 \leq k \leq d,[a]_{p} \in \pi_{p}[\mathcal{D}]
$$

is conservative, for every $p \in \Gamma_{2}$. Now, fix $X:=\sum_{k=1}^{d} c_{k} X_{k} \in \mathfrak{g}, p \in \Gamma$ and $[a]_{p} \in \pi_{p}[\mathcal{D}]$. If $f \in\left(\mathcal{A} / N_{p}\right)^{\prime}$ is a tangent functional at $[a]_{p}$ - in other words, if $f$ is a continuous linear functional on $\mathcal{A} / N_{p}$ such that $\left|f\left([a]_{p}\right)\right|=\|f\|\left\|[a]_{p}\right\|_{p}$ - then by conservativity of the basis elements and [30, Proposition 3.1.14, page 175], it follows that

$$
\operatorname{Re} f\left(\delta_{x, p}\left([a]_{p}\right)\right)=\operatorname{Re} f\left(\left[\delta_{X}(a)\right]_{p}\right)=\sum_{k=1}^{d} c_{k} \operatorname{Re} f\left(\delta_{k, p}\left([a]_{p}\right)\right)=0
$$


so $\delta_{x, p}$ is conservative. Since $X$ and $p$ are arbitrary, this proves $\left.\delta_{X}\right|_{\mathcal{D}}$ is $\Gamma_{2}$-conservative, for every $X \in \mathfrak{g}$. By Theorem 2.12 and Lemma 3.5, this implies $(\mathcal{D}, \mathfrak{g}, \eta)$ exponentiates to a representation by $*$-automorphisms which are $\Gamma_{2}$-isometrically equicontinuous oneparameter groups.

In view of Theorem 3.8, a small upgrade of Theorem 3.7 can be done for locally $\mathrm{C}^{*}$ algebras. More precisely, the hypothesis that the *-derivations $\left\{\delta_{k}\right\}_{1 \leq k \leq d}$ must be $\Gamma$ conservative is not needed, and a theorem in perfect analogy with the $\mathrm{C}^{*}$ one $[26$, Corollary 2] can be obtained:

Theorem 3.9 (Exponentiation - Locally $\mathrm{C}^{*}$-Algebras, II): Let $\mathcal{A}$ be a locally $C^{*}$-algebra, $\mathfrak{g}$ a real finite-dimensional Lie algebra with an ordered basis $\mathcal{B}:=\left(X_{k}\right)_{1 \leq k \leq d}$ and $\left(\mathcal{A}_{\infty}, \mathfrak{g}, \eta\right)$ a representation of $\mathfrak{g}$ by closed $*$-derivations, with

$$
\mathcal{A}_{\infty}:=\bigcap_{n=1}^{+\infty} \bigcap\left\{\operatorname{Dom}\left[\delta_{i_{1}} \ldots \delta_{i_{k}} \ldots \delta_{i_{n}}\right]: 1 \leq k \leq n, 1 \leq i_{k} \leq d\right\},
$$

where $\delta_{X}:=\eta(X), \delta_{k}:=\eta\left(X_{k}\right), 1 \leq k \leq d$, and $\Delta:=\sum_{k=1}^{d} \delta_{k}^{2}$. Then, the following are equivalent:

1. $\left(\mathcal{A}_{\infty}, \mathfrak{g}, \eta\right)$ exponentiates to a strongly continuous locally equicontinuous representation $\alpha: G \longrightarrow \mathcal{L}(\mathcal{A})$ by $*$-automorphisms of a simply connected Lie group $G$, having $\mathfrak{g}$ as its Lie algebra, such that $d \alpha(X)=\overline{\left.\eta(X)\right|_{\mathcal{A}_{\infty}}}$ is the generator of a $\Gamma$ isometrically equicontinuous one-parameter group, for all $X \in \mathfrak{g}$;

2. $\Delta$ is an infinitesimal pregenerator of a $\Gamma$-contractively equicontinuous semigroup $t \longmapsto$ $S(t)$ satisfying $S(t)[\mathcal{A}] \subseteq \mathcal{A}_{\infty}$, for all $t \in(0,1]$ and, for each $p \in \Gamma$, there exists $C_{p}>0$ for which the estimates

$$
\rho_{p, 1}(S(t) a) \leq C_{p} t^{-\frac{1}{2}} p(a)
$$

are verified for all $t \in(0,1]$ and $a \in \mathcal{A}$. 


\section{Bibliography}

[1] Abbaspour, H.; Moskowitz, M., "Basic Lie Theory", World Scientific, 2007;

[2] Albanese, A.A.; Bonet, J.; Ricker, W.J., "Montel Resolvents and Uniformly Mean Ergodic Semigroups of Linear Operators", Quaestiones Mathematicae 36 (2013), 253290 ;

[3] Albanese, A.A.; Jornet, D., "Dissipative Operators and Additive Perturbations in Locally Convex Spaces", Mathematische Nachrichten 289 (2016), 920-949;

[4] Allan, G.R., "A Spectral Theory for Locally Convex Algebras", Proceedings of the London Mathematical Society 15 (1965), 399-421;

[5] Allan, G.R., "On a Class of Locally Convex Algebras", Proceedings of London Mathematical Society 17 (1967), 91-114;

[6] Arens, R., "The Space $L^{\omega}$ and Convex Topological Rings", Bulletin of the American Mathematical Society 52 (1946), 931-935;

[7] Arveson, W., "On Groups of Automorphisms of Operator Algebras", Journal of Functional Analysis 15 (1974), 217-243;

[8] Babalola, V.A., "Semigroups of Operators on Locally Convex Spaces", Transactions of the American Mathematical Society 199 (1974), 163-179;

[9] Bagarello, F.; Fragoulopoulou, M.; Inoue, A.; Trapani, C., "The Completion of a $C^{*}$-Algebra with a Locally Convex Topology", Journal of Operator Theory 56 (2006), 357-376;

[10] Bagarello, F.; Fragoulopoulou, M.; Inoue, A.; Trapani, C., "Locally Convex Quasi C* Normed Algebras", Journal of Mathematical Analysis and Applications 366 (2010), 593-606; 
[11] Barut, A.O.; Raczka, R., "Theory of Group Representations and Applications", PWN - Polish Scientific Publishers, Warszawa, 1980;

[12] Batty, C.J.K., "Small Perturbations of $C^{*}$-Dynamical Systems", Communications in Mathematical Physics 68 (1979), 39-43;

[13] Becker, R., "Derivations on LMC ${ }^{*}$-Algebras", Mathematische Nachrichten 155 (1992), 141-149;

[14] Bers, L.; John, F.; Schechter M., "Partial Differential Equations", Lectures in Applied Mathematics, Vol. III, American Mathematical Society, John Wiley and Sons, 1964;

[15] Bhatt, S.J., "A Note on Generalized B*-Algebras", Journal of the Indian Mathematical Society 43 (1979), 253-257;

[16] Bhatt, S.J., "A Note on Generalized $B^{*}$-Algebras-II", Journal of the Indian Mathematical Society 44 (1980), 285-290;

[17] Bhatt, S.J.,"Topological *-Algebras with $C^{*}$-Enveloping Algebras II", Proceedings of the Indian Academy of Sciences 111 (2001), 65-94;

[18] Bhatt, S.J.; Inoue, A.; Ogi, H., "Differential Structures in $C^{*}$-Algebras", Journal of Operator Theory 66 (2011), 301-334;

[19] Bhatt, S.J.; Karia, D.J.,"Topological *-Algebras with $C^{*}$-Enveloping Algebras", Proceedings of the Indian Academy of Sciences 102 (1992), 201-215;

[20] Bhatt, S.; Karia, D.; Shah, M.M., "On a Class of Smooth Fréchet Subalgebras of $C^{*}$-Algebras", Proceedings of the Indian Academy of Sciences 123 (2013), 393-413;

[21] Bhattacharyya, T.; Dritschel, M.A., "Operator Algebras and Mathematical Physics: 24th International Workshop in Operator Theory and its Applications", Operator Theory: Advances and Applications 247, Birkhäuser, Springer International Publishing Switzerland 2015;

[22] Blackadar, B., "Operator Algebras: Theory of $C^{*}$-Algebras and Von Neumann Algebras", Encyclopedia of Mathematical Sciences 122, Springer-Verlag Berlin Heidelberg, 2006 ;

[23] Blackadar, B.; Cuntz, J., "Differential Banach Algebra Norms and Smooth Subalgebras of $C^{*}$-Algebras", Journal of Operator Theory 26 (1991), 255-282;

[24] Bratteli, O., "Derivations, Dissipations and Group Actions on $C^{*}$-Algebras", Lecture Notes in Mathematics 1229, Springer-Verlag Berlin Heidelberg, 1986; 
[25] Bratteli, O.; Goodman, F.M.; Jorgensen, P.E.T.; Robinson, D.W., "The Heat Semigroup and Integrability of Lie Algebras", Journal of Functional Analysis 79 (1988), 351-397;

[26] Bratteli, O.; Jorgensen, P.E.T., "Conservative Derivations and Dissipative Laplacians", Journal of Functional Analysis 82 (1989), 404-411;

[27] Bratteli, O.; Robinson, D.W., "Unbounded Derivations of $C^{*}$-Algebras", Communications in Mathematical Physics 42 (1975), 253-268;

[28] Bratteli, O.; Robinson, D.W., "Unbounded Derivations of $C^{*}$-Algebras II", Communications in Mathematical Physics 46 (1976), 11-30;

[29] Bratteli O.; Robinson, D.W., "Unbounded Derivations of Von Neumann Algebras", Annales de l'Institut Henri Poincaré XXV (1976), 139-164;

[30] Bratteli, O.; Robinson, D.W., "Operator Algebras and Quantum Statistical Mechanics I", Springer-Verlag Berlin Heidelberg, 2002;

[31] Brezis, H., "Functional Analysis, Sobolev Spaces and Partial Differential Equations", Universitext, Springer Science+Business Media, LLC, 2011;

[32] Cabral, R.A.H.M., "O Teorema Espectral e a Propriedade de "Self-Adjointness" para Alguns Operadores de Schrödinger", Master's Dissertation, Instituto de Matemática e Estatística, Universidade de São Paulo, 2014; http://www.teses.usp.br/teses/disponiveis/45/45132/tde-07032015-154510/pt-br.php (in portuguese);

[33] Cabral, R.A.H.M.; Melo, S.T., "Operators with Analytic Orbit for the Torus Action", Studia Mathematica 243 (2018), 243-250;

[34] Calderón, A.P.; Vaillancourt, R., "On the Boundedness of Pseudo-Differential Operators", Journal of the Mathematical Society of Japan 23 (1971), 374-378;

[35] Casselman, B., "Essays on Representations of Real Groups: The Theorem of Dixmier and Malliavin",

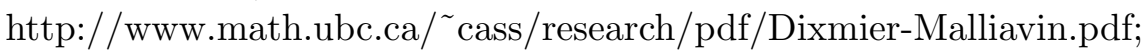

[36] Chernoff, P.R., "Perturbations of Dissipative Operators with Relative Bound One", Proceedings of the American Mathematical Society 33 (1972), 72-74;

[37] Cobos, G., "The Lorentz Group", Treball Final de Grau, Grau de Matemàtiques, Facultat de Matemàtiques, Universitat de Barcelona;

[38] Connes, A. "Classification of injective factors. Cases $I I_{1}, I I_{\infty}, I I I_{\lambda}, \lambda \neq 1$ ", Annals of Mathematics 104 (1976), 73-115; 
[39] Connes, A., "Noncommutative Geometry", Academic Press, 1994;

[40] Conrad, K., "Simplicity of $P S L_{n}(F)$ ", https://kconrad.math.uconn.edu/blurbs/grouptheory/PSLnsimple.pdf

[41] Cordes, H.O., "On Pseudo-Differential Operators and Smoothness of Special Lie-Group Representations", Manuscripta Mathematica 28 (1979), 51-69;

[42] Cordes, H.O., "The Technique of Pseudodifferential Operators", London Mathematical Society Lecture Note Series 202, Cambridge University Press, 1995;

[43] Dixmier, J., "C $C^{*}$-Algebras", North-Holland Publishing Company, 1977;

[44] Dixmier, J., "Von Neumann Algebras", North-Holland Publishing Company, 1981;

[45] Dixmier, J.; Malliavin, P., "Factorizations of Infinitely Differentiable Functions and Smooth Vectors", https://www.yumpu.com/en/document/view/30811272/factorizations-of-infinitelydifferentiable-functions-and-gvsu;

[46] Dixon, P.G., "Generalized $B^{*}$-Algebras", Proceedings of The London Mathematical Society 21 (1970), 693-715;

[47] Dixon, P.G., "Generalized B*-Algebras II", Journal of the London Mathematical Society 5 (1972), 159-165;

[48] Elliott, G.A.; Li, H., "Morita Equivalence of Smooth Noncommutative Tori", Acta Mathematica 199 (2007), 1-27;

[49] Ter Elst, A.F.M.; Robinson, D.W., "Elliptic Operators on Lie Groups", Acta Aplicandae Mathematicae 44 (1996), 133-150;

[50] Engel, K.-J.; Nagel, R., "One-Parameter Semigroups for Linear Evolution Equations", Graduate Texts in Mathematics 194, Springer-Verlag New York, Inc., 2000;

[51] Folland, G.B., "Fourier Analysis and its Applications", Brooks/Cole Publishing Company, Wadsworth Inc., 1992;

[52] Forger, M.; Römer, H., "Elementary Field Theory" (online), VCH Verlag, Weinheim, 1993 ;

[53] Fragoulopoulou, M., "Topological Algebras with Involution", Elsevier B. V., 2005;

[54] Fragoulopoulou, M.; Inoue, A.; Kürsten, K.-D., "Old and New Results on Allan's GB*Algebras", Banach Center Publications 91 (2010), 169-178; 
[55] Fragoulopoulou, M.; Weigt, M.; Zarakas I., "Derivations of Locally Convex *-Algebras", Extracta Mathematicae 26 (2011), 45-60;

[56] Gallier, J.; Quaintance, J., "Notes on Differential Geometry and Lie Groups" (online notes), University of Pennsylvania, 2018;

[57] Gårding, L., "Note on Continuous Representations of Lie Groups", Proceedings of the National Academy of Sciences of the United States of America 33 (1947), 331-332;

[58] Goodman, R., "Analytic and Entire Vectors for Representations of Lie Groups", Transactions of the American Mathematical Society 143 (1969), 55-76;

[59] Goodman, F.M.; Jorgensen, P.E.T., "Lie Algebras of Unbounded Derivations", Journal of Functional Analysis 52 (1983), 369-384;

[60] Graff, R.A., "A Simple Theory of Differential Calculus in Locally Convex Spaces", Transactions of the American Mathematical Society 293 (1986), 485-509;

[61] Gustafson, K., "A Perturbation Lemma", Bulletin of the American Mathematical Society 72 (1966), 334-338;

[62] Haag, R., "Local Quantum Physics: Fields, Particles, Algebras", Springer-Verlag Berlin Heidelberg, 1996;

[63] Haagerup, U., "Connes' bicentralizer problem and uniqueness of the injective factor of type $I I I_{1} "$, Acta Mathematica 158 (1987), 95-148;

[64] Halmos, P.R., "Measure Theory", Springer-Verlag New York Inc., 1974;

[65] Harish-Chandra, "Representations of a Semisimple Lie Group on a Banach Space. I", Transactions of the American Mathematical Society 75 (1953), 185-243;

[66] Helemskii, A.Ya., "The Homology of Banach and Topological Algebras", Kluwer Academic Publishers, 1989;

[67] Hilgert, J.; Neeb, K.-H., "Structure and Geometry of Lie Groups", Springer Science+Business Media, LLC, 2012;

[68] Hille, E.; Phillips, R.S. "Functional Analysis and Semi-groups", American Mathematical Society 31, 1957;

[69] Hoffman, K.; Kunze, R., "Linear Algebra", Prentice-Hall, Inc., 1971;

[70] Inoue, A., "Locally $C^{*}$-Algebra", Memoirs of the Faculty of Science, Kyushu University 25 (1971), 197-235; 
[71] Inoue, A.; Kürsten, K.-D., "On $C^{*}$-Like Locally Convex *-Algebras", Mathematische Nachrichten 235 (2002), 51-58;

[72] Jarchow, H., "Locally Convex Spaces", B.G. Teubner Stuttgart, 1981;

[73] Jorgensen, P.E.T., "Operators and Representation Theory", Mathematics Studies 147, North-Holland, Elsevier Science Publishers B.V., 1988;

[74] Jorgensen, P.E.T.; Moore, R.T., "Operator Commutation Relations", D. Reidel Publishing Company, 1984;

[75] Jost, J., "Partial Differential Equations", Graduate Texts in Mathematics 214, Springer-Verlag New York, Inc., 2002;

[76] Kissin, E.; Shulman, V.S., "Dense Q-Subalgebras of Banach and $C^{*}$-Algebras and Unbounded Derivations of Banach and $C^{*}$-Algebras", Proceedings of the Edinburgh Mathematical Society 36 (1993), 261-276;

[77] Knapp, A.W., "Lie Groups Beyond an Introduction", Progress in Mathematics 140, Birkhäuser, 2002;

[78] Komura, T., "Semigroups of Operators in Locally Convex Spaces", Journal of Functional Analysis 2 (1968), 258-296;

[79] Lance, E.C., "Hilbert C*-Modules: a Toolkit for Operator Algebraists", London Mathematical Society Lecture Note Series 210, Cambridge University Press, 1995;

[80] Langlands, R.P., "Semi-groups and Representations of Lie Groups", PhD Thesis, Yale University, 1960;

[81] Langlands, R.P.; Robinson, D., "The Work of Robert Langlands", Institute for Advanced Study (online);

http://publications.ias.edu/rpl/section $/ 3$

[82] Lee, G.-S., "Review of Differential Geometry" (online notes), https://www.mathi.uni-heidelberg.de/ ${ }^{\sim}$ lee/GonencSS16.pdf;

[83] Leoni, G. "A First Course in Sobolev Spaces", Graduate Studies in Mathematics 105, American Mathematical Society, Providence, Rhode Island, 2009;

[84] Longo, R., "On Perturbed Derivations of $C^{*}$-algebras", Reports on Mathematical Physics 12 (1977), 119-124;

[85] Longo, R., "Automatic Relative Boundedness of Derivations in $C^{*}$-Algebras", Journal of Functional Analysis 34 (1979), 21-28; 
[86] MacCluer, B., "Elementary Functional Analysis", Springer Science+Business Media, LLC, 2009;

[87] Magyar, Z., "On the Analytic Vector Variant of the Hille-Yosida Theorem", Mathematica Scandinavica 65 (1989), 93-102;

[88] Magyar, Z., "Heat Kernels on Lie Groups", Journal of Functional Analysis 93 (1990), 351-390;

[89] Melo, S.T., "Characterizations of Pseudodifferential Operators on the Circle", Proceedings of the American Mathematical Society 125 (1997), 1407-1412;

[90] Michael, E.A., "Locally Multiplicatively-Convex Topological Algebras", Memoirs of the American Mathematical Society 11, Providence, Rhode Island, 1952;

[91] Moore, R.T., "Measurable, Continuous and Smooth Vectors for Semigroups and Group Representations", Memoirs of the American Mathematical Society 78, Providence, Rhode Island, 1968;

[92] Munkres, J.R., "Topology", Prentice Hall Inc., 2000;

[93] Murphy, G.J., "C $C^{*}$-Algebras and Operator Theory", Academic Press, Inc., 1990;

[94] Nelson, E., "Analytic Vectors", Annals Of Mathematics 70 (1959), 572-615;

[95] Nelson, E.; Stinespring, W.F., "Representation of Elliptic Operators in an Enveloping Algebra", American Journal of Mathematics 81 (1959), 547-560;

[96] Nirenberg, L., "Remarks on Strongly Elliptic Partial Differential Equations", Communications on Pure and Applied Mathematics VIII (1955), 648-674;

[97] Ouchi, S., "Semi-groups of operators in locally convex spaces", Journal of the Mathematical Society of Japan 25 (1973), 265-276;

[98] Palmer, T.W., "Banach Algebras and the General Theory of *-Algebras, Volume I: Algebras and Banach Algebras", Encyclopedia of Mathematics and its Applications 49, Cambridge University Press, 1994;

[99] Pedersen, G.K., "C $C^{*}$-Algebras and their Automorphism Groups", Academic Press Inc., 1979 ;

[100] Phillips, N.C., "Inverse Limits of $C^{*}$-Algebras", Journal of Operator Theory 19 (1988), 159-195;

[101] Phillips, N.C., "Inner Derivations on $\sigma-C^{*}$-Algebras", Mathematische Nachrichten 176 (1995), 243-247; 
[102] Poulsen, N.S., "On $C^{\infty}$-Vectors and Intertwining Bilinear Forms for Representations of Lie Groups", Journal of Functional Analysis 9 (1972), 87-120;

[103] Powers, R.T.; Sakai, S., "Existence of Ground States and KMS States for Approximately Inner Dynamics", Communications in Mathematical Physics 39 (1975), 273288 ;

[104] Reed, M.; Simon, B., "Methods of Modern Mathematical Physics I: Functional Analysis", Academic Press, Inc., 1980;

[105] Reed, M.; Simon, B., "Methods of Modern Mathematical Physics II: Fourier Analysis, Self-Adjointness", Academic Press, Inc., 1975;

[106] Ribes, L.; Zalesskii, P., "Profinite Groups", A Series of Modern Surveys in Mathematics 40, Springer-Verlag Berlin Heidelberg, 2010;

[107] Rieffel, M.A., "Deformation Quantization for Actions of $\mathbb{R}^{d "}$, Memoirs of the American Mathematical 106, 1993;

[108] Robinson, D.W., "The Heat Semigroup and Integrability of Lie Algebras: Lipschitz Spaces and Smoothness Properties", Communications in Mathematical Physics 132 (1990), 217-243;

[109] Robinson, D.W., "Elliptic Operators and Lie Groups", Oxford University Press, 1991;

[110] Rudin, W., "Functional Analysis", McGraw-Hill, Inc., 1991;

[111] Rusinek, J., "Analytic Vectors and Generation of One-Parameter Groups", Studia Mathematica LXXIX (1984), 77-82;

[112] Rusinek, J., "Analytic Vectors and Integrability of Lie Algebra Representations", Journal of Functional Analysis 74 (1987), 10-23;

[113] Ruzhansky, M.; Turunen, V., "Pseudo-Differential Operators and Symmetries", Birkhäuser Verlag AG, 2010;

[114] Sakai, S., "C $C^{*}$-Algebras and $W^{*}$-Algebras", Springer-Verlag Berlin Heidelberg, 1971;

[115] Sakai, S., "Recent Developments in the Theory of Unbounded Derivations in $C^{*}$. Algebras", Proceedings of the International Congress of Mathematicians, Helsinki (1978), 709-714;

[116] Sakai, S., "Operator Algebras in Dynamical Systems", Encyclopedia of Mathematics and its Applications 41, Cambridge University Press, 1991.

[117] Schaefer, H.H., "Topological Vector Spaces", Springer-Verlag New York, 1971; 
[118] Schmüdgen, K., "Unbounded Operator Algebras and Representation Theory", Operator Theory: Advances and Applications 37, Springer Basel AG, 1990;

[119] Schweitzer, L.B., "A Short Proof that $M_{n}(\mathcal{A})$ is Local if $\mathcal{A}$ is Local and Fréchet", International Journal of Mathematics 3 (1992), 581-589;

[120] Takesaki, M., "Theory of Operator Algebras I", Springer-Verlag New York Inc., 1979;

[121] Trèves, F., "Topological Vector Spaces, Distributions and Kernels", Academic Press, Inc., 1967

[122] Warner, G., "Harmonic Analysis on Semi-Simple Lie Groups I", Springer-Verlag Berlin Heidelberg, 1972;

[123] Weigt, M.; Zarakas, I., "Derivations of Generalized B*-Algebras", Extracta Mathematicae 28 (2013), 77-94;

[124] Weigt, M.; Zarakas, I., "Derivations of Fréchet Nuclear GB*-Algebras", Bulletin of the Australian Mathematical Society 92 (2015), 290-301;

[125] Yngvason, J., "The role of type III factors in quantum field theory", Reports on Mathematical Physics 55 (2005), 135-147;

[126] Yosida, K., "Functional Analysis", Springer-Verlag Berlin Heidelberg, 1980. 